ORAU provides innovative scientific and technical solutions to advance research and education, protect public health and the environment and strengthen national security. Through specialized teams of experts, unique laboratory capabilities and access to a consortium of more than 100 major Ph.D.-granting institutions, ORAU works with federal, state, local and commercial customers to advance national priorities and serve the public interest. A 501(c)(3) nonprofit corporation and federal contractor, ORAU manages the Oak Ridge Institute for Science and Education (ORISE) for the U.S. Department of Energy (DOE). Learn more about ORAU at www.orau.org.

\section{NOTICES}

The opinions expressed herein do not necessarily reflect the opinions of the sponsoring institutions of Oak Ridge Associated Universities.

This report was prepared as an account of work sponsored by the United States Government. Neither the United States Government nor the U.S. Department of Energy, nor any of their employees, makes any warranty, expressed or implied, or assumes any legal liability or responsibility for the accuracy, completeness, or usefulness of any information, apparatus, product, or process disclosed, or represents that its use would not infringe on privately owned rights. Reference herein to any specific commercial product, process, or service by trade name, mark, manufacturer, or otherwise, does not necessarily constitute or imply its endorsement or recommendation, or favor by the U.S. Government or any agency thereof. The views and opinions of authors expressed herein do not necessarily state or reflect those of the U.S. Government or any agency thereof. 


\title{
DATA SHARING REPORT FOR THE QUANTIFICATION OF REMOVABLE ACTIVITY IN VARIOUS SURVEILLANCE AND MAINTENANCE PROJECT FACILITIES AT THE OAK RIDGE NATIONAL LABORATORY OAK RIDGE, TENNESSEE
}

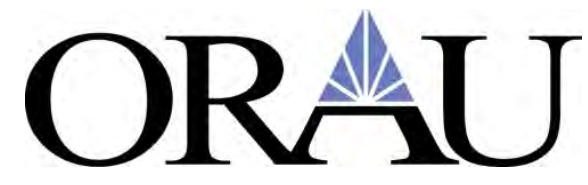

\author{
Prepared by \\ David A. King \\ Independent Environmental Assessment and Verification Program \\ Oak Ridge Associated Universities
}

December 2013

Prepared for the

U.S. Department of Energy 


\section{DATA SHARING REPORT FOR THE QUANTIFICATION OF REMOVABLE ACTIVITY IN VARIOUS SURVEILLANCE AND MAINTENANCE PROJECT FACILITIES AT THE OAK RIDGE NATIONAL LABORATORY OAK RIDGE, TENNESSEE}

Prepared by:

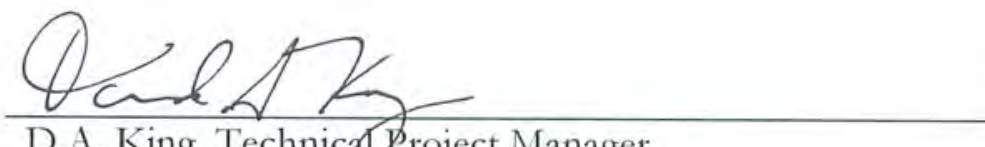

Date: $12 / 18 / 2613$

D.A. King, Technical Project Manager

Independent Environmental Assessment and Verification Program

Reviewed by:

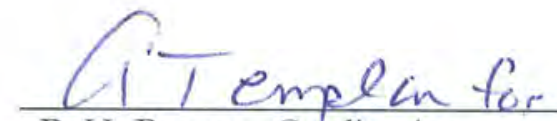

P. H. Benton, Quality Assurance Specialist Independent Environmental Assessment and Verification Program

Reviewed and approved for release by:

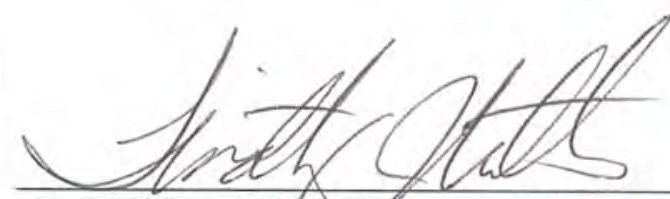

T. J. Vitkus, IEAy yssociate Director Date:

Date: $12 / 18 / 13$ Independent Environmental Assessment and Verification Program 


\section{CONTENTS}

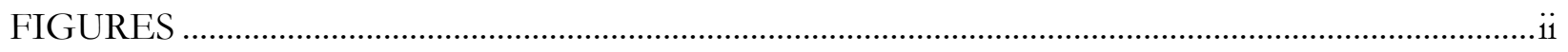

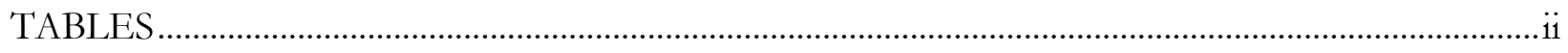

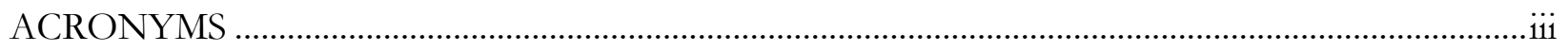

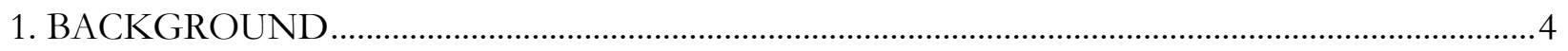

2. SAMPLE AND ANALYSIS METHODS _..................................................................................

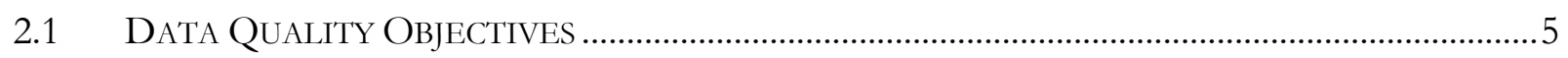

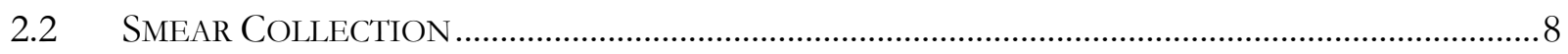

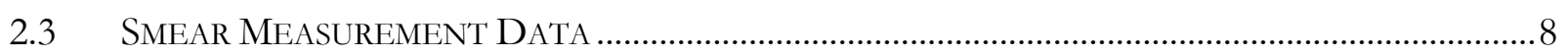

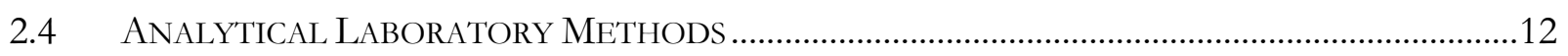

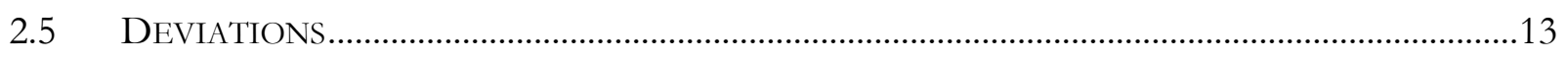

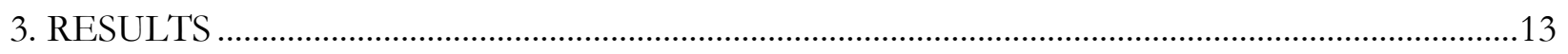

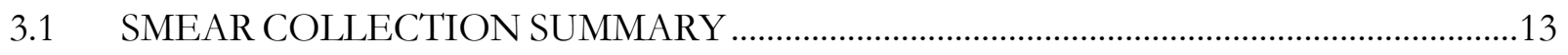

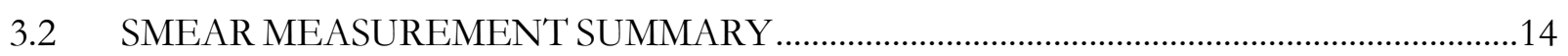

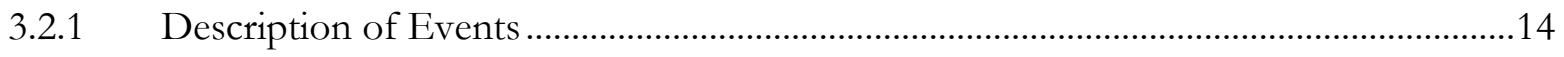

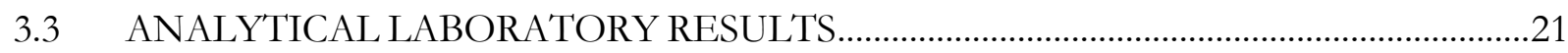

3.4 SMEAR MEASUREMENT AND ANALYTICAL RESULTS.............................................23

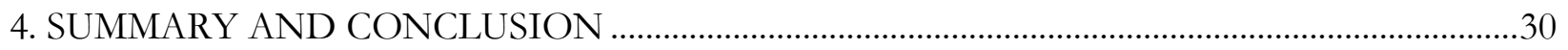

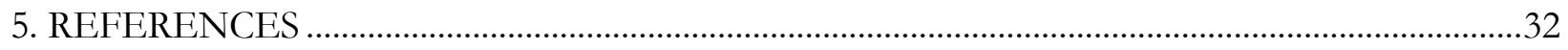

APPENDIX A. FACILITY MAPS SHOWING SMEAR LOCATIONS

APPENDIX B. PHOTOGRAPHS OF SMEAR LOCATIONS

APPENDIX C. STATISTICAL SUMMARY OF LABORATORY ANALYTICAL RESULTS

APPENDIX D. PLOTS OF LABORATORY ANALYTICAL DATA VS. INSTRUMENT MEASUREMENT DATA

APPENDIX E. LIVING MEMORY SUMMARIES

APPENDIX F. HAZARDOUS MATERIALS OF CONCERN 


\section{FIGURES}

Fig. 2.1. Representative Instruments Used for Surveying and Smear Data Collection ......................... 9

Fig. 2.2. Fume Hood Used to Collect Radiation Measurement Data ...................................................11

Fig. 2.3. Representative Smears Collected During the Investigation ....................................................11

Fig. 3.1. Relationship Between ORAU and UCOR Instrument Responses-Alpha ..........................20

Fig. 3.2. Relationship Between ORAU and UCOR Instrument Responses-Beta..............................21

Fig. 3.3. Alpha cpm vs. pCi/sample of Am-241 in the Reactor Building Group ................................27

Fig. 3.4. Beta cpm vs. pCi/sample of Cs-137 in the Reactor Building Group .....................................28

Fig. 3.5. Alpha cpm vs. pCi/sample of Cm-243/244 in the Non-reactor Building Group.................29

Fig. 3.6. Beta cpm vs. pCi/sample of Cs-137 in the Non-reactor Building Group ...............................30

\section{TABLES}

Table 2.1. Smear Measurement Instrumentation and Source Information............................................. 9

Table 2.2. Analytical Methods and Target Detection Limits for Radiological SRCs...........................12

Table 3.1. Summary of Smear Sampling and Analysis Activities ...........................................................14

Table 3.2. Summary of Smear Measurement Data and Laboratory Analysis Decisions .......................16

Table 3.3. Summary of Laboratory Analytical Data for All Facilities Combined.................................23 


\section{ACRONYMS}

$\begin{array}{ll}\text { ARRA } & \text { American Recovery and Reinvestment Act } \\ \text { cpm } & \text { counts per minute } \\ \text { DOE } & \text { U.S. Department of Energy } \\ \text { dpm } & \text { disintegrations per minute } \\ \text { DQO } & \text { data quality objective } \\ \text { OR-EM } & \text { Oak Ridge Office of Environmental Management } \\ \text { EMWMF } & \text { Environmental Management Waste Management Facility } \\ \text { LAW } & \text { large-area wipe } \\ \text { ORAU } & \text { Oak Ridge Associated Universities } \\ \text { ORISE } & \text { Oak Ridge Institute for Science and Education } \\ \text { ORNL } & \text { Oak Ridge National Laboratory } \\ \text { PEMS } & \text { Project Environmental Measurements System } \\ \text { PPE } & \text { personal protective equipment } \\ \text { RCT } & \text { Radiation Control Technician } \\ \text { S\&M } & \text { surveillance and maintenance } \\ \text { SAP } & \text { sampling and analysis plan } \\ \text { SMO } & \text { Sample Management Office } \\ \text { SRC } & \text { site-related contaminant } \\ \text { UCOR } & \text { URS |CH2M Oak Ridge, LLC } \\ \text { WAC } & \text { waste acceptance criteria } \\ \text { WHP } & \text { waste handling plan } \\ \text { WL } & \text { waste lot }\end{array}$




\section{ORAU}

\section{DATA SHARING REPORT FOR THE QUANTIFICATION OF REMOVABLE ACTIVITY IN VARIOUS SURVEILLANCE AND MAINTENANCE PROJECT FACILITIES AT THE OAK RIDGE NATIONAL LABORATORY OAK RIDGE, TENNESSEE}

\section{BACKGROUND}

The U.S. Department of Energy (DOE) Oak Ridge Office of Environmental Management (OR-EM) requested that Oak Ridge Associated Universities (ORAU), working under the Oak Ridge Institute for Science and Education (ORISE) contract, provide technical and independent waste management planning support using American Recovery and Reinvestment Act (ARRA) funds. Specifically, DOE OR-EM requested that ORAU plan and implement a sampling and analysis campaign targeting potential removable radiological contamination that may be transferrable to future personal protective equipment (PPE) and contamination control materials-collectively referred to as PPE throughout the remainder of this report-used in certain URS|CH2M Oak Ridge, LLC (UCOR) Surveillance and Maintenance (S\&M) Project facilities at the Oak Ridge National Laboratory (ORNL).

Routine surveys in Bldgs. 3001, 3005, 3010, 3028, 3029, 3038, 3042, 3517, 4507, and 7500 continuously generate PPE. The waste is comprised of Tyvek coveralls, gloves, booties, Herculite, and other materials used to prevent worker exposure or the spread of contamination during routine maintenance and monitoring activities. This report describes the effort to collect and quantify removable activity that may be used by the ORNL S\&M Project team to develop radiation instrumentation "screening criteria." Material potentially containing removable activity was collected on smears, including both masselin large-area wipes (LAWs) and standard paper smears, and analyzed for site-related constituents (SRCs) in an analytical laboratory. The screening criteria, if approved, may be used to expedite waste disposition of relatively clean PPE. The ultimate objectives of this effort were to: 1) determine whether screening criteria can be developed for these facilities, and 2) provide process knowledge information for future site planners. The screening criteria, if calculated, must be formally approved by Federal Facility Agreement parties prior to use for ORNL S\&M Project PPE disposal at the Environmental Management Waste Management Facility (EMWMF). 
ORAU executed the approved sampling and analysis plan (SAP) (DOE 2013) while closely coordinating with ORNL S\&M Project personnel and using guidelines outlined in the Waste Handling Plan for Surveillance and Maintenance Activities at the Oak Ridge National Laboratory, DOE/OR/01-2565\&D2 (WHP) (DOE 2012). WHP guidelines were followed because the PPE waste targeted by this SAP is consistent with that addressed under the approved Waste Lot (WL) 108.1 profile for disposal at EMWMF—-this PPE is a "future waste stream" as defined in the WHP. The SAP presents sampling strategy and methodology, sample selection guidelines, and analytical guidelines and requirements necessary for characterizing future ORNL S\&M Project PPE waste. This report presents a review of the sample and analysis methods including data quality objectives (DQOs), required deviations from the original design, summary of field activities, radiation measurement data, analytical laboratory results, a brief presentation of results, and process knowledge summaries.

Due to time and access constraints, Bldgs. 3005, 3038, and 7500 could not be accessed by project personnel. However, ORNL S\&M Project personnel provided ORAU with 100 standard paper smears that had been collected during routine surveys of Bldg. 3038. Additional details are presented later in this report.

\section{SAMPLE AND ANALYSIS METHODS}

The following subsections present a summary of the DQOs established in the SAP, a summary of the smear collection and radiation measurement methods, and the analytical methods used to quantify radionuclide-specific concentration on each smear submitted for laboratory analysis. A summary of necessary deviations from the SAP are presented at the end of this section.

\subsection{Data Quality OBJEctives}

The SAP presents DQOs developed per Guidance on Systematic Planning Using the Data Quality Objectives Process (EPA 2006) to specify the quality of data required to support decisions during the investigation. The seven DQO steps that were developed and applied to this investigation are summarized in the following discussion. 
Step 1-State the Problem. The problem is that the ORNL S\&M Project would like to develop facility-specific screening criteria to streamline PPE waste disposition activities that satisfy either the EMWMF bounding profile WL 108.1 or alternate disposal facility requirements.

ORNL S\&M Project personnel generate a large quantity of PPE—-mostly Tyvek coveralls, gloves, and booties - that may become contaminated if it contacts a surface with removable radioactive material. Current waste management practices include the storage and eventual sampling of stockpiled PPE waste for disposition at EMWMF. It may be possible; however, to develop screening criteria that will expedite the disposition of PPE generated during future ORNL S\&M Project activities. The criteria would represent a radiation measurement triggering either a sampling requirement (eventually) or acceptance without sampling under the WL 108.1 profile. Specifically, the conceived screening criterion for a given facility would be a radiation measurement in units of counts per minute $(\mathrm{cpm})$ or disintegrations per minute per $100 \mathrm{~cm}^{2}\left(\mathrm{dpm} / 100 \mathrm{~cm}^{2}\right)$ that corresponds to acceptable SRC concentrations in units of $\mathrm{pCi} / \mathrm{g}$. The data collected per the SAP will be used to develop, if possible, facility-specific screening criteria and to increase the process knowledge of target facilities.

Step 2-Identify the Decision/Objective. The decision statement for the investigation is as follows:

Determine, through a sufficient evaluation of process knowledge, radiation measurement, and laboratory analytical data, whether facility-specific screening criteria may be developed and used for the streamlined disposition of future ORNL S\&M Project PPE waste; or whether the ORNL S\&M Project must continue current practices for disposal under EMWMF WL profile 108.1 including volumetric sampling and laboratory analysis.

Step 3-Identify Information Inputs. Smears were collected at locations known to contain removable contamination from accessible target facilities. These smears were subject to direct radiation measurement in the field and in a controlled environment, and then a subset were submitted for laboratory analysis for SRCs including uranium, thorium, transuranics, fission products, and activation products. Inputs included existing process knowledge resources available prior to the onset of field activities; existing approved procedures and protocols for data collection, 
analytical laboratories, and data quality; and facility walkdowns plus interviews with knowledgeable site personnel such as Radiation Control Technicians (RCTs) with facility-specific experience.

Step 4-Define the Boundaries. The target population consisted of surface removable radiological contamination in Bldgs. 3001, 3005, 3010, 3028, 3029, 3038, 3042, 3517, 4507 and 7500. The spatial constraints included the facilities themselves and safety-related access restrictions. Temporal constraints included the required conclusion of fieldwork (September 30, 2013) and the reporting deadline set by ORAU's contract period of performance close (December 31, 2013).

Step 5-Develop the Analytical Approach. The parameters of interest are concentrations in $\mathrm{pCi} / \mathrm{g}$ or $\mathrm{pCi} / \mathrm{sample}$, and detector responses in $\mathrm{cpm}$ or $\mathrm{dpm} / 100 \mathrm{~cm}^{2}$. A range of values is minimally required; thus, at least two smears were to be collected per facility. For cases where a facility had multiple potential SRC distributions (based on operational history), two samples per operational area were collected, if possible. Ideally, detector response ranges were to span from relatively small values (i.e., representing a proportionally small amount of contamination) to large values (i.e., a large amount of contamination).

Step 6- Specific Performance or Acceptance Criteria. A consistent set of calibrated and functionally stable radiation measurement instrumentation were used for the duration of the field investigation. All laboratory analyses were performed by a DOECAP-approved laboratory selected by the Oak Ridge Sample Management Office (SMO). The objective was to achieve detection limits at $10 \%$ or less of the applicable EMWMF waste acceptance criteria (WAC) or the method detection limit, whichever was larger. Additionally, data quality was to be such that the SMO validations would not result in the rejection (R-flag) of any laboratory analytical results.

Step 7- Develop the Plan for Obtaining Data. Data collection for this investigation required coordination between ORNL S\&M Project and ORAU personnel. Ideally, ORNL S\&M Project personnel (e.g., an RCT) would identify locations in the designated facility (or operational area) known to, or suspected to, contain removable radiological contamination. ORAU personnel collected instrument-specific count data on each smear. The results of full suite radiological analyses were reported in $\mathrm{pCi} /$ sample and have been provided to ORNL S\&M Project personnel. Instrument responses (i.e., measurement data) were also submitted to ORNL S\&M Project personnel. 


\subsection{SMEAR COLLECTION}

Access to target facilities was coordinated through respective UCOR facility managers. Specific smear locations were identified through a combination of historical data reviews (e.g., previous survey maps provided by RCTs), discussions with UCOR personnel, and scans with ORAU radiation detection instrumentation. Scanning was specifically used to refine smear locations and confirm that elevated radiation levels were present at the selected location. In some cases; however, high background radiation levels detected in an area precluded effective field screening. In those cases the smears were collected as planned and scanned in a relatively low background area to assure that removable contamination was transferred to the sample medium. Details on radiation detection instrumentation are presented in Sect. 2.3 of this report.

LAWs, the preferred smear medium, were collected using a full size masselin sheet folded into a 6inch $\times 6$-inch square. One side of the square was labeled with a permanent marker which included the building number, RCT-defined contamination area number, and sequential smear number. For example, the label 3001-41-001 represents Bldg. 3001, area 41, and smear number 1. The label served as both the smear number and the sample number, as applicable. The other side of the 6-inch $\times 6$-inch square was used to perform a gross wipe of the target location. As stated above, the material transferred to the masselin was scanned using radiation instruments to verify the presence of contamination. The masselin sample was placed in a one gallon zip-top bag and stored in a custody-controlled cooler for transport to the ORAU/ORISE Radiological and Environmental Analysis Laboratory facility on Bethel Valley Road in Oak Ridge, Tennessee.

\subsection{SMEAR MEASUREMENT DATA}

Per the SAP, smear-specific radiation measurement data were collected in a controlled environment using a repeatable and systematic approach. UCOR provided ORAU investigators with a set of instruments that could be used during future routine ORNL S\&M Project activities. ORAU used the same set of instruments that were used during the fieldwork phase of the project, as described in Sect. 2.2. Figure 2.1 illustrates representative radiation measurement instruments. 


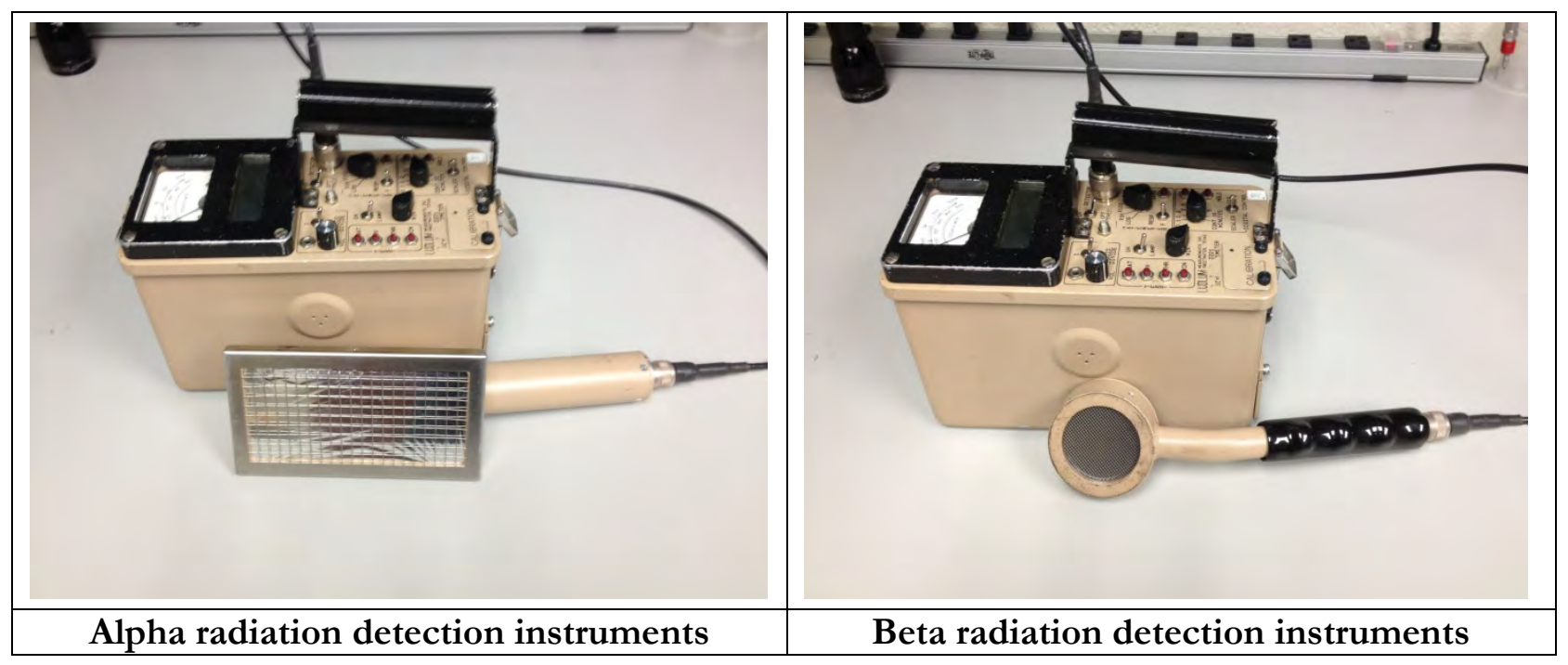

Fig. 2.1. Representative Instruments Used for Surveying and Smear Data Collection

Operational checkout parameters for all instruments were established at the ORAU/ORISE Bethel Valley Road facility for each detector/ratemeter combination presented in Table 2.1. The listed check sources were used to establish acceptable detector operational response ranges when exposed to the detector specific target radiation-i.e. alpha or beta-gamma. Operational response ranges in the absence of a radiation source (i.e., background) were also established in the same fume hood used to count smears. Operational checkouts were performed each day of use prior to and after counting smears to ensure the instruments responded within acceptable performance parameters.

\begin{tabular}{|c|c|c|c|c|c|}
\hline \multirow{2}{*}{$\begin{array}{l}\text { Radiation } \\
\text { Type }\end{array}$} & \multicolumn{2}{|c|}{ ORAU Instruments } & \multicolumn{2}{|c|}{ UCOR Instruments } & \multirow{2}{*}{$\begin{array}{l}\text { Check } \\
\text { Source }\end{array}$} \\
\hline & Detector & Ratemeter & Detector & Ratemeter & \\
\hline Alpha & $\begin{array}{l}\text { Model 43-92 } \\
\text { scintillator } \\
\text { Inst. no. } 671\end{array}$ & $\begin{array}{l}\text { Model } 2221 \\
\text { Inst. no. } 380\end{array}$ & $\begin{array}{l}\text { Model 43-93 } \\
\text { scintillator } \\
\text { Inst. no. PR236947 }\end{array}$ & $\begin{array}{l}\text { Model } 2360 \\
\text { Inst. no. } \\
225196\end{array}$ & $\begin{array}{l}\text { Th-230 } \\
\text { no. } 471\end{array}$ \\
\hline Beta & $\begin{array}{l}\text { Model 44-9 GM } \\
\text { Inst. no. } 913\end{array}$ & $\begin{array}{l}\text { Model } 2221 \\
\text { Inst. no. } 338\end{array}$ & $\begin{array}{l}\text { Model 44-9 GM } \\
\text { Inst. no. Y75300 }\end{array}$ & $\begin{array}{l}\text { Model } 12 \\
\text { Inst. no. } \\
207275\end{array}$ & $\begin{array}{l}\text { Sr- } 90 \\
\text { no. } 440\end{array}$ \\
\hline
\end{tabular}

All detectors and ratemeters manufactured by Ludlum

$\mathrm{GM}=$ Geiger-Mueller

Prior to counting smears, a clean piece of plastic sheeting was placed on the hood's work surface; pictured in Fig. 2.2. A masselin LAW was carefully removed from the zip-top container and unfolded so the label and smeared material faced up, as pictured in Fig. 2.3. The label and smeared 
material were photographed, a one-minute static count was performed for each detector detector/ratemeter combination listed in Table 2.1, and results were tabulated. Then the masselin was refolded, placed back in the zip-top container, and returned to the sample cooler. The technician scanned the plastic sheeting to verify contamination had not been transferred from the LAW, and the process was repeated with the next LAW. These steps were repeated until measurement data were tabulated from all LAWs.

For the 100 standard paper smears obtained from UCOR for Bldg. 3038, the RCTs' survey reports were used to separate the standard smears into groups of six smears collected from adjacent locations. Six standard smears were selected for two reasons. First, the SMO laboratory indicated six would be sufficient to achieve the detection limits listed in Table 2.2. Second, six standard smears laid side-by-side in a 2-smear $\times 3$-smear pattern (as shown in Fig. 2.3) generally matched the effective surface area of the masselin smears. The selected six- standard-smear composites were submitted for laboratory analysis after static measurement data were collected.

The project allowed for up to 40 total samples to be submitted for laboratory analysis. Smears from eight facilities were available for submission and the DQOs required the establishment of an overall range of detector responses for a given facility. There were not enough samples to represent a range for each region of interest from within each facility; therefore, investigators targeted the smears that produced the maximum alpha and/or beta radiation responses and smears that produced low, but still distinguishable from background, detector responses. With the range of radiation responses represented, investigators selected smears from all RCT-defined contamination areas and smears producing mid-range detector responses. This process resulted in a list of proposed samples, which was presented to ORNL S\&M Project management for consideration; the list was approved without alterations. Therefore, 40 samples were submitted to a SMO-approved laboratory for analysis, as planned. 


\section{ORAU}

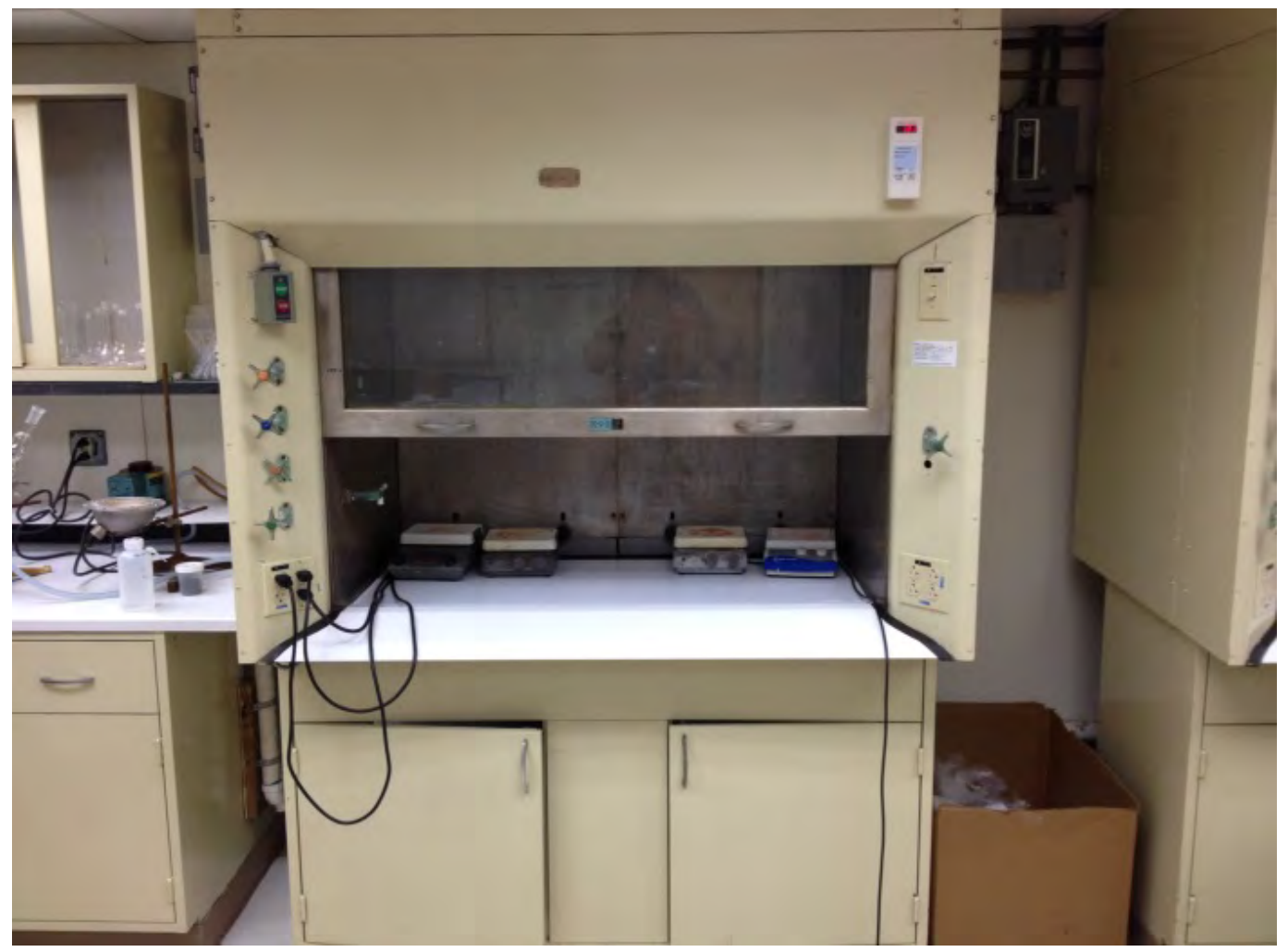

Fig. 2.2. Fume Hood Used to Collect Radiation Measurement Data

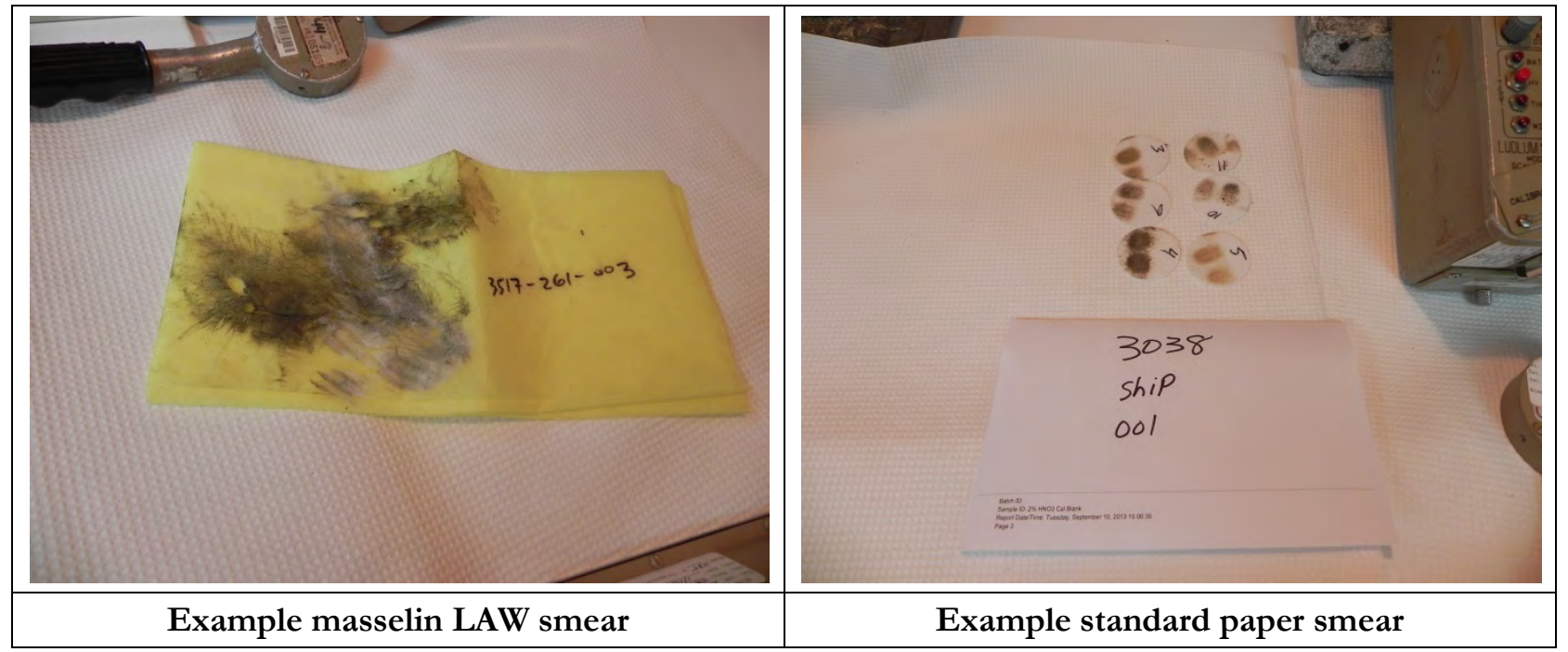

Fig. 2.3. Representative Smears Collected During the Investigation 


\subsection{ANALYTICAL LABORATORY METHODS}

Samples were collected and laboratory analyses were performed in order to characterize removable radioactive material on submitted smears. The specific parameters and analytes listed in Table 2.2 were chosen based on 1) process knowledge; 2) known facility-specific SRCs; and 3) the parameters required to meet the EWMWF WAC. All analyses and quality control requirements were performed in accordance with this SAP and the SMO-approved laboratory statement of work. The laboratory performed the sample preparation and analysis in accordance with requirements of applicable EPA methods, laboratory procedures, and/or the statement of work, as appropriate. Listed target detection limits were below target detection limits presented in the SAP.

\section{Table 2.2. Analytical Methods and Target Detection Limits for Radiological SRCs}

\begin{tabular}{|c|c|c|c|c|c|c|c|}
\hline Method & Analyte & MDL & Units & Container & Preservative & $\begin{array}{l}\text { Minimum } \\
\text { Sample } \\
\text { Size }\end{array}$ & $\begin{array}{l}\text { Hold } \\
\text { Time }\end{array}$ \\
\hline \multirow[t]{2}{*}{$\begin{array}{l}\text { gamma } \\
\text { spec. }\end{array}$} & $\begin{array}{l}\text { Am-243, Co-60, } \\
\text { I-129, Cs-137, } \\
\text { Eu-152, Eu-154, } \\
\text { Ra-226 }\end{array}$ & $1^{\mathrm{a}}$ & $\mathrm{pCi} / \mathrm{g}$ & Plastic bag & None & $200 \mathrm{~g}$ & $180 \mathrm{~d}$ \\
\hline & $\mathrm{Pa}-231$ & $5^{\mathrm{a}}$ & $\mathrm{pCi} / \mathrm{g}$ & Plastic bag & None & $200 \mathrm{~g}$ & $180 \mathrm{~d}$ \\
\hline GPC & Sr-90 & 1 & $\mathrm{pCi} / \mathrm{g}$ & Plastic bag & None & $200 \mathrm{~g}$ & $180 \mathrm{~d}$ \\
\hline \multirow[t]{4}{*}{ liquid scint. } & $\mathrm{H}-3$ & 10 & $\mathrm{pCi} / \mathrm{g}$ & Plastic bag & None & $200 \mathrm{~g}$ & $180 \mathrm{~d}$ \\
\hline & C-14 & 5 & $\mathrm{pCi} / \mathrm{g}$ & Plastic bag & None & $200 \mathrm{~g}$ & $180 \mathrm{~d}$ \\
\hline & $\mathrm{Ni}-63$ & 2 & $\mathrm{pCi} / \mathrm{g}$ & Plastic bag & None & $200 \mathrm{~g}$ & $180 \mathrm{~d}$ \\
\hline & Tc-99 & 1 & $\mathrm{pCi} / \mathrm{g}$ & Plastic bag & None & $200 \mathrm{~g}$ & $180 \mathrm{~d}$ \\
\hline \multirow[t]{6}{*}{ alpha spec. } & Am-241 & 0.1 & $\mathrm{pCi} / \mathrm{g}$ & Plastic bag & None & $200 \mathrm{~g}$ & $180 \mathrm{~d}$ \\
\hline & Isotopic Cm & 0.4 & & & & & \\
\hline & Isotopic $\mathrm{Pu}$ & 0.1 & & & & & \\
\hline & Isotopic Th & 0.2 & & & & & \\
\hline & Isotopic U & 0.1 & & & & & \\
\hline & $\mathrm{Np}-237$ & 0.1 & & & & & \\
\hline
\end{tabular}

${ }^{a}$ Detection limits for gamma spec. are assumed to be an average over at least 200 grams.

Short-lived radionuclides or radionuclides that do not have prescribed concentration limits for either carcinogenic

WAC or auditable safety analysis WAC are not included in this tabulation

$\mathrm{GPC}=$ gas proportional counting

$\mathrm{MDL}=$ minimum detection limit

$\mathrm{N} / \mathrm{A}=$ not applicable 


\subsection{DEVIATIONS}

Access to specific sections of some facilities was prevented by UCOR and/or ORAU management due to personnel safety concerns or because of the potential for an unacceptable risk of releasing radiological materials. Included in this list were: the ventilation system in Bldg. 3001; the "pump room" in the Bldg. 3042 basement; and the high contaminated areas in Bldg. 3029, 4507, and 3517.

ORAU personnel were unable to access three of the identified facilities prior to the DOE-imposed close of fieldwork on September 30, 2013. The deteriorated condition of Bldg. 3005 precluded entry and no smear samples were collected. Building 7500 posed a non-radiological hazard from exposure to asbestos which would require extensive planning to address worker safety concerns that could not be completed prior to the September 30 deadline. Construction in Bldg. 3038 also precluded entry by ORAU personnel although recent surveys by UCOR RCTs generated 100 standard paper smear samples that were transferred to ORAU for measurement and, if appropriate, laboratory analysis.

\section{RESULTS}

The following subsections present a summary of field activities, smear-specific static measurement data, analytical laboratory data for submitted smears, and a high-level analysis of the relationship between static measurement and laboratory analytical data.

\subsection{SMEAR COLLECTION SUMMARY}

Table 3.1 lists the number of smears collected per building and which smears were submitted for laboratory analysis. As shown, ORAU personnel collected 72 LAWs from seven facilities. Building 3038 could not be accessed, but the ORNL S\&M Project provided 100 standard smears from recent routine surveys-some of these were batched into 6-standard-smear composites and submitted for laboratory analysis. Buildings 3005 and 7500 could not be accessed during the field phase of the project and no alternate smears could be provided.

Appendix A presents plan-view illustrations of target facilities, and the approximate location where each smear was collected. Appendix B presents photographs for each location for smears collected by ORAU investigators. The caption above each photograph describes the physical location, and the 
parenthetical "Sampled" indicates that the smear was submitted for laboratory analysis. ORAU could not access Bldg. 3038, so no photographs are provided.

\begin{tabular}{ccccc}
\hline \multicolumn{5}{c}{ Table 3.1. Summary of Smear Sampling and Analysis Activities } \\
\hline Facility No. & $\begin{array}{c}\text { Sampling } \\
\text { Organization }\end{array}$ & $\begin{array}{c}\text { Smears } \\
\text { Collected }\end{array}$ & $\begin{array}{c}\text { Smear } \\
\text { Medium }\end{array}$ & $\begin{array}{c}\text { Smears Submitted } \\
\text { for Analysis }\end{array}$ \\
\hline 3001 & ORAU & 11 & Masselin & 4 \\
3005 & N/A & None & N/A & N/A \\
3010 & ORAU & 10 & Masselin & 4 \\
3028 & ORAU & 10 & Masselin & 6 \\
3029 & ORAU & 10 & Masselin & 6 \\
3038 & UCOR/S\&M & 100 & Standard paper & 5 (batches of 6) \\
3042 & ORAU & 11 & Masselin & 5 \\
3517 & ORAU & 10 & Masselin & 5 \\
4507 & ORAU & 10 & Masselin & 5 \\
7500 & N/A & None & N/A & N/A \\
\hline
\end{tabular}

$\mathrm{N} / \mathrm{A}=$ not applicable; access restrictions precluded data collection

\subsection{SMEAR MEASUREMENT SUMMARY}

\subsubsection{Description of Events}

Table 3.2 presents smear-specific direct measurement data. These results are organized by facility but are sorted by net beta measurement results as defined by the UCOR model 44-9 instrument response. This sort was selected for three reasons: 1) beta responses are the more dominant; 2) UCOR (not ORAU) instruments will be used to make future ORNL S\&M Project decisions; and 3) a sort based on beta-response is preferred over the arbitrary sample ID for selecting smears for laboratory analysis. The table presents gross and net detector responses, where net is defined as the gross response minus the average background response. For example, the net beta response for smear number 3001-1239-001 is the gross response of 84 counts per minute (cpm) minus the average background response of $42.5 \mathrm{cpm}$ for a net of 41.5 or $\sim 42 \mathrm{cpm}$. Net values are presented here as integers for aesthetics only. Graphical analyses presented later in this report use all significant digits. The UCOR instrument produced the maximum allowable beta response of $200,000 \mathrm{cpm}$ when counting smear 3001-16-004, thus the smear was not considered for laboratory analysis. This is because the detector may have significantly under-responded to contaminants and a potential relationship between detector response and concentration may not be quantified. 
Values listed in Table 3.2 were not converted from cpm to dpm given efficiencies, used in the conversion, vary by instrument and are not required during this stage of the investigation. UCOR may incorporate instrument-specific conversions as part of their screening criteria analysis.

Fig. 3.1 illustrates the relationship between ORAU detector responses (x-axis) and UCOR detector responses (y-axis) for alpha-detecting instruments. The dashed line represents a theoretical one-toone relationship, where perfect agreement would be depicted by all points falling on the line. However, the detector response ratio of measured values should span, but still track along, the theoretical line. This is indeed the case shown in Fig. 3.1, indicating good agreement between ORAU and UCOR instrument responses.

Fig. 3.2 illustrates the relationship between ORAU detector responses (x-axis) and UCOR detector responses (y-axis) for beta-detecting instruments. This figure also suggests good agreement between ORAU and UCOR instrument responses, though the UCOR detector over-responds at low $(<10 \mathrm{cpm})$ radiation levels relative to the ORAU instrument. This should not be a concern to UCOR investigators unless screening criteria, if calculated, are on the order of tens of cpm beta. Even then, the screening criteria would be calculated for UCOR instruments and the ORAU responses are primarily offered as quality control to assure UCOR instruments do not grossly under-respond or over-respond to contamination. The conclusion is that the UCOR instruments reasonably respond to both alpha- and beta-emitting contaminants, though an upper bound of 200,000 cpm beta must be considered during screening criteria calculations. 


\section{ORA}

\begin{tabular}{|c|c|c|c|c|c|c|c|c|c|c|}
\hline \multirow[b]{3}{*}{ Bldg. } & \multirow[b]{3}{*}{ Smear ID } & \multicolumn{4}{|c|}{ ORAU Instruments } & \multicolumn{4}{|c|}{ UCOR Instruments } & \multirow[b]{3}{*}{$\begin{array}{c}\text { Lab. } \\
\text { Analysis }\end{array}$} \\
\hline & & \multicolumn{2}{|c|}{ Model 43-92 - Alpha } & \multicolumn{2}{|c|}{ Model 44-9 - Beta } & \multicolumn{2}{|c|}{ Model 43-93 - Alpha } & \multicolumn{2}{|c|}{ Model 44-9 - Beta } & \\
\hline & & $\begin{array}{c}\text { Gross } \\
\text { cpm }\end{array}$ & $\begin{array}{l}\text { Net } \\
\text { cpm }\end{array}$ & $\begin{array}{c}\text { Gross } \\
\text { cpm }\end{array}$ & $\begin{array}{l}\text { Net } \\
\text { cpm }\end{array}$ & $\begin{array}{c}\text { Gross } \\
\text { cpm }\end{array}$ & $\begin{array}{l}\text { Net } \\
\text { cpm }\end{array}$ & $\begin{array}{c}\text { Gross } \\
\text { cpm }\end{array}$ & $\begin{array}{l}\text { Net } \\
\text { cpm }\end{array}$ & \\
\hline \multirow[t]{11}{*}{3001} & $3001-1239-001$ & 3 & 1 & 58 & 14 & 2 & 2 & 84 & 42 & $\mathrm{NO}$ \\
\hline & $3001-1239-002$ & 2 & 0 & 147 & 103 & 1 & 1 & 113 & 71 & YES \\
\hline & 3001-16-007 & 4 & 2 & 89 & 45 & 7 & 7 & 122 & 80 & $\mathrm{NO}$ \\
\hline & $3001-16-003$ & 1 & 0 & 130 & 86 & 0 & 0 & 141 & 99 & $\mathrm{NO}$ \\
\hline & $3001-16-002$ & 5 & 3 & 161 & 117 & 4 & 4 & 184 & 142 & $\mathrm{NO}$ \\
\hline & $3001-16-005$ & 9 & 7 & 411 & 367 & 11 & 11 & 252 & 210 & YES \\
\hline & 3001-16-006 & 6 & 4 & 368 & 324 & 4 & 4 & 283 & 241 & $\mathrm{NO}$ \\
\hline & 3001-16-001 & 12 & 10 & 434 & 390 & 6 & 6 & 548 & 506 & $\mathrm{NO}$ \\
\hline & $3001-16-009$ & 0 & 0 & 491 & 447 & 4 & 4 & 604 & 562 & YES \\
\hline & 3001-16-008 & 11 & 9 & 1589 & 1545 & 4 & 4 & 1536 & 1494 & YES \\
\hline & 3001-16-004 & 1962 & 1960 & 294,411 & 294,367 & 1249 & 1249 & $200,000+$ & - & $\mathrm{NO}$ \\
\hline \multirow[t]{10}{*}{3010} & $3010-41-003$ & 3 & 1 & 84 & 40 & 0 & 0 & 107 & 65 & $\mathrm{NO}$ \\
\hline & 3010-41-001 & 0 & 0 & 500 & 456 & 1 & 1 & 474 & 432 & YES \\
\hline & $3010-41-009$ & 3 & 1 & 632 & 588 & 0 & 0 & 680 & 638 & $\mathrm{NO}$ \\
\hline & $3010-41-002$ & 3 & 1 & 593 & 549 & 1 & 1 & 720 & 678 & $\mathrm{NO}$ \\
\hline & $3010-41-007$ & 1 & 0 & 1005 & 961 & 0 & 0 & 1221 & 1179 & $\mathrm{NO}$ \\
\hline & $3010-41-010$ & 1 & 0 & 1138 & 1094 & 0 & 0 & 1398 & 1356 & YES \\
\hline & $3010-41-008$ & 0 & 0 & 1731 & 1687 & 4 & 4 & 1972 & 1930 & $\mathrm{NO}$ \\
\hline & $3010-41-006$ & 0 & 0 & 1760 & 1716 & 0 & 0 & 2121 & 2079 & $\mathrm{NO}$ \\
\hline & $3010-41-004$ & 2 & 0 & 2233 & 2189 & 0 & 0 & 2231 & 2189 & YES \\
\hline & $3010-41-005$ & 2 & 0 & 3784 & 3740 & 0 & 0 & 4222 & 4180 & YES \\
\hline \multirow[t]{3}{*}{3028} & 3028-87-002 & 34 & 32 & 56 & 12 & 33 & 33 & 55 & 13 & $\mathrm{NO}$ \\
\hline & 3028-79-003 & 98 & 96 & 57 & 13 & 121 & 121 & 57 & 15 & YES \\
\hline & $3028-87-003$ & 500 & 498 & 47 & 3 & 232 & 232 & 66 & 24 & YES \\
\hline
\end{tabular}




\section{ORA}

\begin{tabular}{|c|c|c|c|c|c|c|c|c|c|c|}
\hline \multirow[b]{3}{*}{ Bldg. } & \multirow[b]{3}{*}{ Smear ID } & \multicolumn{4}{|c|}{ ORAU Instruments } & \multicolumn{4}{|c|}{ UCOR Instruments } & \multirow[b]{3}{*}{$\begin{array}{c}\text { Lab. } \\
\text { Analysis }\end{array}$} \\
\hline & & \multicolumn{2}{|c|}{ Model 43-92 - Alpha } & \multicolumn{2}{|c|}{ Model 44-9 - Beta } & \multicolumn{2}{|c|}{ Model 43-93 - Alpha } & \multicolumn{2}{|c|}{ Model 44-9 - Beta } & \\
\hline & & $\begin{array}{c}\text { Gross } \\
\text { cpm }\end{array}$ & $\begin{array}{l}\text { Net } \\
\text { cpm }\end{array}$ & $\begin{array}{c}\text { Gross } \\
\text { cpm }\end{array}$ & $\begin{array}{l}\text { Net } \\
\text { cpm }\end{array}$ & $\begin{array}{c}\text { Gross } \\
\text { cpm }\end{array}$ & $\begin{array}{l}\text { Net } \\
\text { cpm }\end{array}$ & $\begin{array}{c}\text { Gross } \\
\text { cpm }\end{array}$ & $\begin{array}{l}\text { Net } \\
\text { cpm }\end{array}$ & \\
\hline 3028 & 3028-79-005 & 14 & 12 & 60 & 16 & 14 & 14 & 66 & 24 & $\mathrm{NO}$ \\
\hline \multirow[t]{6}{*}{ cont. } & 3028-79-004 & 60 & 58 & 53 & 9 & 50 & 50 & 73 & 31 & YES \\
\hline & 3028-87-004 & 69 & 67 & 77 & 33 & 51 & 51 & 74 & 32 & YES \\
\hline & 3028-87-001 & 13 & 11 & 81 & 37 & 15 & 15 & 83 & 41 & $\mathrm{NO}$ \\
\hline & $3028-87-005$ & 481 & 479 & 86 & 42 & 518 & 518 & 91 & 49 & YES \\
\hline & 3028-79-002 & 178 & 176 & 64 & 20 & 142 & 142 & 112 & 70 & $\mathrm{NO}$ \\
\hline & 3028-79-001 & 3280 & 3278 & 106 & 62 & 2247 & 2247 & 172 & 130 & YES \\
\hline \multirow[t]{10}{*}{3029} & 3029-98-004 & 2 & 0.4 & 156 & 112 & 3 & 3 & 93 & 51 & $\mathrm{NO}$ \\
\hline & 3029-95-001 & 27 & 25 & 94 & 50 & 22 & 22 & 94 & 52 & YES \\
\hline & 3029-95-002 & 1 & 0 & 107 & 63 & 3 & 3 & 97 & 55 & YES \\
\hline & $3029-98-002$ & 3 & 1.4 & 97 & 53 & 4 & 4 & 101 & 59 & $\mathrm{NO}$ \\
\hline & 3029-98-006 & 5 & 3.4 & 175 & 131 & 6 & 6 & 120 & 78 & YES \\
\hline & 3029-98-008 & 1 & 0 & 165 & 121 & 5 & 5 & 174 & 132 & YES \\
\hline & 3029-98-005 & 2 & 0.4 & 231 & 187 & 3 & 3 & 180 & 138 & $\mathrm{NO}$ \\
\hline & $3029-98-003$ & 2 & 0.4 & 331 & 287 & 3 & 3 & 391 & 349 & $\mathrm{NO}$ \\
\hline & 3029-98-001 & 0 & 0 & 1443 & 1399 & 1 & 1 & 1560 & 1518 & YES \\
\hline & 3029-98-007 & 1 & 0 & 10,619 & 10,575 & 3 & 3 & 9304 & 9262 & YES \\
\hline \multirow[t]{5}{*}{3038} & 3038-NL-001 & 3 & 1 & 72 & 28 & 8 & 8 & 51 & 9 & YES \\
\hline & 3038-AHF-001 & 32 & 30 & 68 & 24 & 21 & 21 & 78 & 36 & YES \\
\hline & 3038-SL-002 & 10 & 8 & 63 & 19 & 14 & 14 & 91 & 49 & YES \\
\hline & 3038-SL-001 & 96 & 94 & 158 & 114 & 96 & 96 & 144 & 102 & YES \\
\hline & 3038-SHIP-001 & 3 & 1 & 158 & 114 & 9 & 9 & 186 & 144 & YES \\
\hline \multirow[t]{2}{*}{3042} & 3042-181-001 & 0 & 0 & 66 & 22 & 2 & 2 & 71 & 29 & YES \\
\hline & $3042-170-002$ & 1 & 0 & 67 & 23 & 4 & 4 & 77 & 35 & $\mathrm{NO}$ \\
\hline
\end{tabular}




\section{ORA}

\begin{tabular}{|c|c|c|c|c|c|c|c|c|c|c|}
\hline \multirow[b]{3}{*}{ Bldg. } & \multirow[b]{3}{*}{ Smear ID } & \multicolumn{4}{|c|}{ ORAU Instruments } & \multicolumn{4}{|c|}{ UCOR Instruments } & \multirow[b]{3}{*}{$\begin{array}{c}\text { Lab. } \\
\text { Analysis }\end{array}$} \\
\hline & & \multicolumn{2}{|c|}{ Model 43-92 - Alpha } & \multicolumn{2}{|c|}{ Model 44-9 - Beta } & \multicolumn{2}{|c|}{ Model 43-93 - Alpha } & \multicolumn{2}{|c|}{ Model 44-9 - Beta } & \\
\hline & & $\begin{array}{c}\text { Gross } \\
\text { cpm }\end{array}$ & $\begin{array}{l}\text { Net } \\
\text { cpm }\end{array}$ & $\begin{array}{c}\text { Gross } \\
\text { cpm }\end{array}$ & $\begin{array}{l}\text { Net } \\
\text { cpm }\end{array}$ & $\begin{array}{c}\text { Gross } \\
\text { cpm }\end{array}$ & $\begin{array}{l}\text { Net } \\
\text { cpm }\end{array}$ & $\begin{array}{c}\text { Gross } \\
\text { cpm }\end{array}$ & $\begin{array}{l}\text { Net } \\
\text { cpm }\end{array}$ & \\
\hline 3042 & 3042-178-001 & 2 & 0 & 158 & 114 & 1 & 1 & 186 & 144 & YES \\
\hline \multirow[t]{8}{*}{ cont. } & 3042-170-001 & 37 & 35 & 170 & 126 & 30 & 30 & 268 & 226 & $\mathrm{NO}$ \\
\hline & 3042-175-001 & 6 & 4 & 470 & 426 & 2 & 2 & 515 & 473 & YES \\
\hline & 3042-178-002 & 5 & 3 & 416 & 372 & 0 & 0 & 526 & 484 & $\mathrm{NO}$ \\
\hline & $3042-177-001$ & 15 & 13 & 1523 & 1479 & 11 & 11 & 1762 & 1720 & YES \\
\hline & $3042-170-005$ & 4 & 2 & 5061 & 5017 & 7 & 7 & 4620 & 4578 & $\mathrm{NO}$ \\
\hline & 3042-170-003 & 67 & 65 & 10,696 & 10,652 & 58 & 58 & 9820 & 9778 & $\mathrm{NO}$ \\
\hline & 3042-170-004 & 42 & 40 & 39,668 & 39,624 & 43 & 43 & 25,752 & 25,710 & $\mathrm{NO}$ \\
\hline & 3042-170-006 & 706 & 704 & 83,587 & 83,543 & 551 & 551 & 41,091 & 41,049 & YES \\
\hline \multirow[t]{10}{*}{3517} & $3517-266-004$ & 2 & 0.4 & 83 & 39 & 6 & 6 & 88 & 46 & $\mathrm{NO}$ \\
\hline & $3517-266-002$ & 4 & 2.4 & 164 & 120 & 2 & 2 & 189 & 147 & $\mathrm{NO}$ \\
\hline & 3517-266-005 & 8 & 6.4 & 114 & 70 & 5 & 5 & 201 & 159 & YES \\
\hline & $3517-266-001$ & 2 & 0.4 & 164 & 120 & 4 & 4 & 210 & 168 & $\mathrm{NO}$ \\
\hline & $3517-266-003$ & 6 & 4.4 & 220 & 176 & 5 & 5 & 241 & 199 & YES \\
\hline & $3517-261-003$ & 0 & 0 & 911 & 867 & 10 & 10 & 998 & 956 & YES \\
\hline & $3517-261-005$ & 2 & 0.4 & 2533 & 2489 & 8 & 8 & 2618 & 2576 & $\mathrm{NO}$ \\
\hline & $3517-261-004$ & 0 & 0 & 5167 & 5123 & 4 & 4 & 5285 & 5243 & $\mathrm{NO}$ \\
\hline & $3517-261-002$ & 6 & 4.4 & 7515 & 7471 & 7 & 7 & 6399 & 6357 & YES \\
\hline & $3517-261-001$ & 3 & 1.4 & 9111 & 9067 & 7 & 7 & 7548 & 7506 & YES \\
\hline \multirow[t]{5}{*}{4507} & 4507-1874-002 & 4 & 2.4 & 63 & 19 & 5 & 5 & 48 & 6 & $\mathrm{NO}$ \\
\hline & 4507-1874-005 & 1 & 0 & 66 & 22 & 5 & 5 & 63 & 21 & $\mathrm{NO}$ \\
\hline & 4507-1926-001 & 1 & 0 & 50 & 6 & 5 & 5 & 71 & 29 & YES \\
\hline & 4507-1874-001 & 6 & 4.4 & 74 & 30 & 4 & 4 & 78 & 36 & $\mathrm{NO}$ \\
\hline & 4507-1874-003 & 5 & 3.4 & 101 & 57 & 5 & 5 & 86 & 44 & YES \\
\hline
\end{tabular}




\section{ORA}

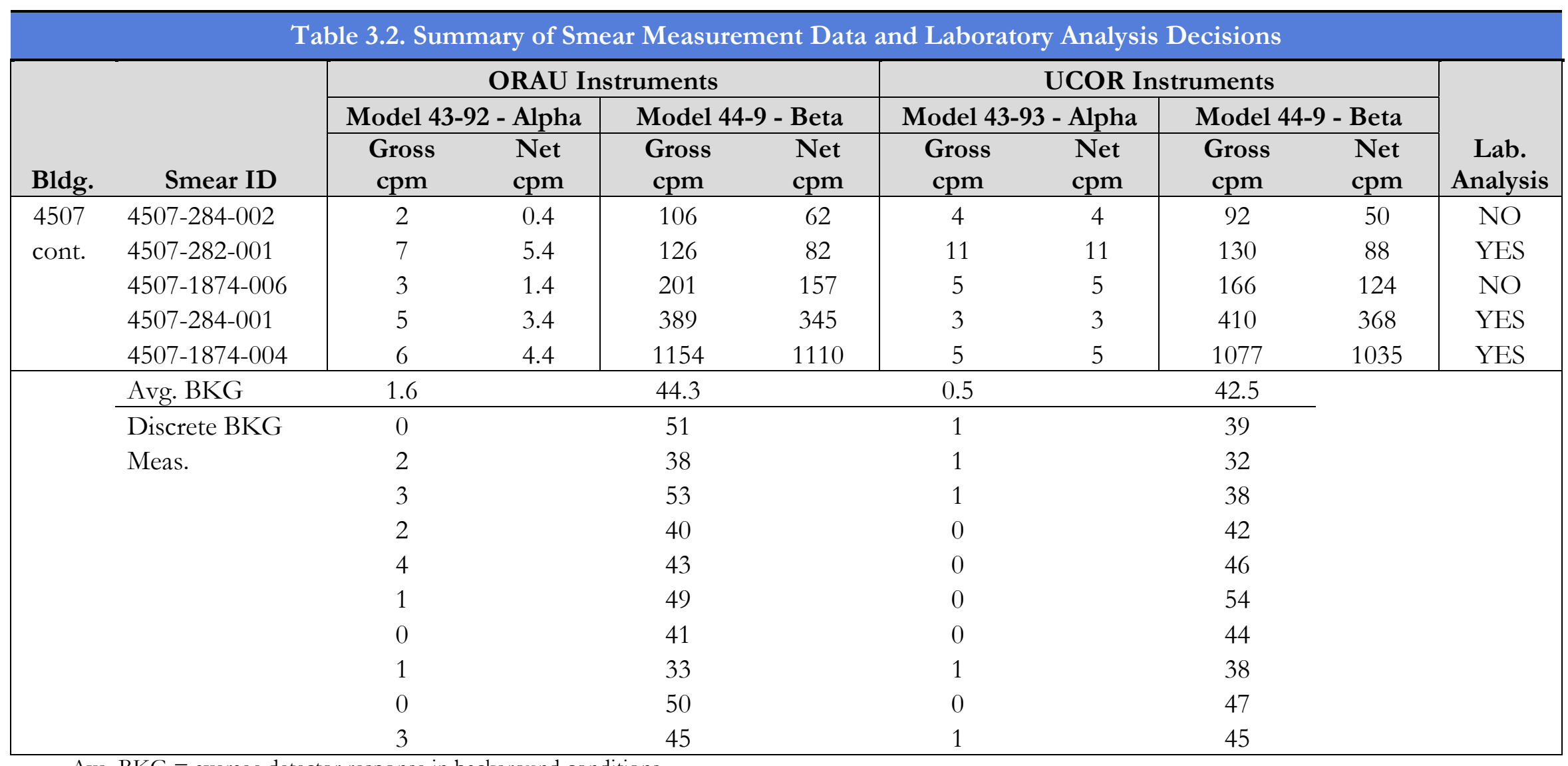

Avg. $\mathrm{BKG}=$ average detector response in background conditions 


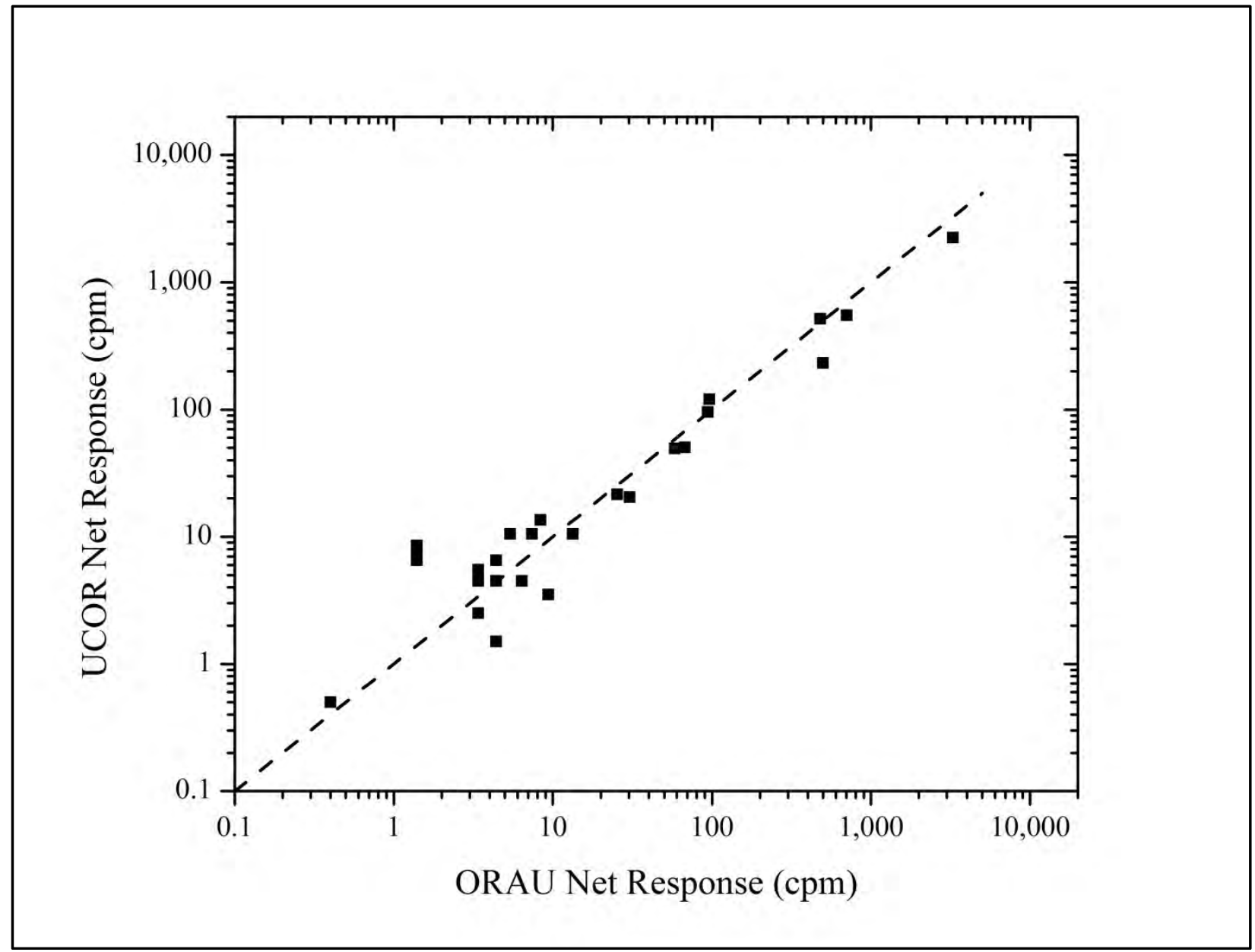

Fig. 3.1. Relationship Between ORAU and UCOR Instrument Responses-Alpha 


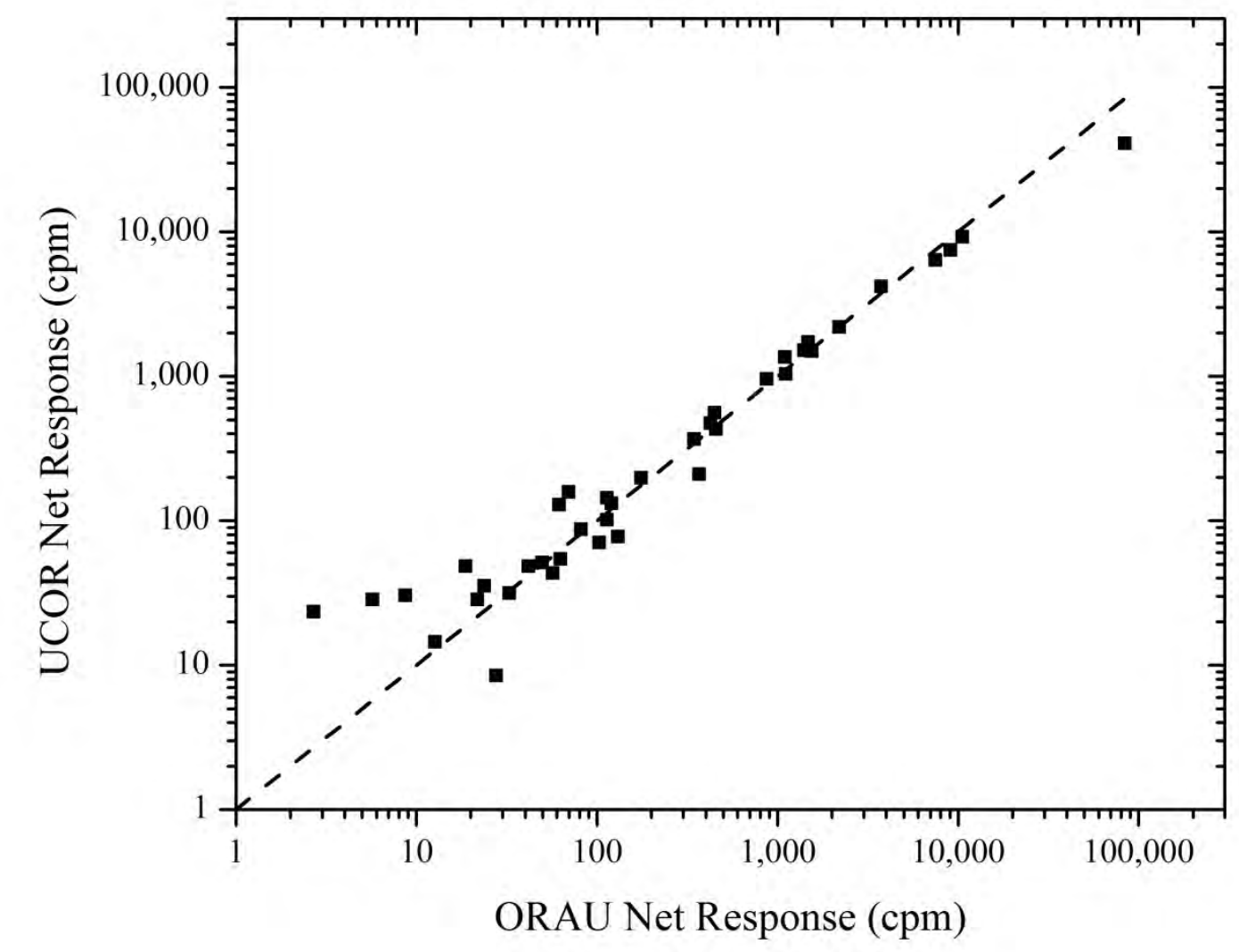

Fig. 3.2. Relationship Between ORAU and UCOR Instrument Responses-Beta

\subsection{ANALYTICAL LABORATORY RESULTS}

Appendix C presents a facility-specific statistical summary of analytical laboratory results. Listed parameters include the analyte (i.e., radionuclide) name; frequency of detection; and the detected minimum, maximum, mean, and standard deviation values. Results for 24 analytes are reported including isotopes of americium, carbon, cesium, cobalt, curium, europium, hydrogen (i.e., tritium), iodine, neptunium, nickel, plutonium, protactinium, radium, technetium, thorium, and uranium. Note $\mathrm{Sr}-90$ is reported as total radioactive strontium, given Sr-90 is the most likely form of strontium associated with facility operations.

All laboratory analytical data were verified and Level-III validated by the SMO. No data were rejected. Analytical results for Am-241 were reported by the analytical laboratory using both gamma 
spectroscopy and alpha spectroscopy methods for some samples. As a general rule, alpha spectroscopy, which involves element-specific chemical separation, provides lower detection limits and associated data generally produce the more representative values. The alpha spectroscopic values are, therefore, used in the App. C summary. All analytical results are available in the Project Environmental Measurements System (PEMS) under the project name "ORNL ISO," are managed and maintained by the SMO, and have been provided electronically to key ORNL S\&M Project representatives.

As a reminder, these data represent only those facilities and areas within facilities that were accessible by ORAU investigators and only the removable fraction of contamination. It is possible that the fixed fraction of contamination contains a different mixture of radionuclides. Also, analytical results are reported as $\mathrm{pCi} /$ sample, though it is assumed the activity could be averaged over a PPE article, such as a Tyvek suit. For a Tyvek suit example, the results would be interpreted as $\mathrm{pCi} / 200$ grams. This scenario assumes contamination was transferred to a small portion of the suit during routine S\&M project activities, and the balance of the suit contains no detectable contamination.

Table 3.3 presents the same data used on App. C but blocked into one large population independent of facility. Note that statistics are presented for detected analytes only. Results indicate the primary removable contaminants are the fission products Cs-137 and Sr-90 with high frequency of detection and reported concentrations. The most prominent alpha emitters are Am-241 and Cm-243/244 considering frequency of detection and reported concentrations combined. Thorium-230 was detected in the majority of samples but at low activity levels. The radionuclides Np-237, Pa-231, and Th-232 were not detected in any of the samples and several other radionuclides were detected in fewer than 10 samples. These results demonstrate that a few long-lived fission products dominate the total population, followed by a few transuranics. 
Table 3.3. Summary of Laboratory Analytical Data for All Facilities Combined

\begin{tabular}{|l|c|c|c|c|c|c|}
\hline \multirow{2}{*}{\multicolumn{1}{|c|}{ Radionuclide }} & \multirow{2}{*}{$\begin{array}{c}\text { Freq. of } \\
\text { Detect }\end{array}$} & \multicolumn{5}{c|}{ Statistics for Detections (pCi/sample) } \\
\cline { 5 - 7 } & $25 / 40$ & 2.49 & 3620 & 208 & 12.3 & 729 \\
\hline Americium-241 & $1 / 40$ & 2.92 & 2.92 & 2.92 & 2.92 & - \\
\hline Americium-243 & $5 / 40$ & 236 & 3130 & 1110 & 620 & 1200 \\
\hline Carbon-14 & $35 / 40$ & 6.81 & 427,000 & 15,500 & 177 & 72,200 \\
\hline Cesium-137 & $8 / 40$ & 19.4 & 22,000 & 3240 & 452 & 7610 \\
\hline Cobalt-60 & $3 / 40$ & 1.82 & 9.26 & 6.44 & 8.24 & 4.03 \\
\hline Curium-242 & $19 / 40$ & 2.70 & 15,900 & 1140 & 14.6 & 3630 \\
\hline Curium-243/244 & $2 / 40$ & 236 & 75,500 & 37,900 & 37,900 & 53,200 \\
\hline Europium-152 & $1 / 40$ & 19,400 & 19,400 & 19,400 & 19,400 & - \\
\hline Europium-154 & $4 / 40$ & 4.31 & 453 & 121 & 13.7 & 221 \\
\hline Iodine-129 & $0 / 40$ & - & - & - & - & - \\
\hline Neptunium-237 & $12 / 40$ & 172 & 36,900 & 5670 & 669 & 11,900 \\
\hline Nickel-63 & $13 / 40$ & 1.61 & 1210 & 175 & 22.2 & 351 \\
\hline Plutonium-238 & $12 / 40$ & 3.20 & 111 & 32.4 & 15.8 & 32.9 \\
\hline Plutonium-239/240 & $0 / 40$ & - & - & - & - & - \\
\hline Protactinium-231 & $28 / 40$ & 25.1 & 12,200 & 1610 & 186 & 3390 \\
\hline Radioactive Strontium (Total) & $1 / 40$ & 92.2 & 92.2 & 92.2 & 92.2 & \\
\hline Radium-226 & $17 / 40$ & 5.72 & 2960 & 358 & 8.65 & 790 \\
\hline Technetium-99 & $29 / 40$ & 2.17 & 24.50 & 5.20 & 4.40 & 3.93 \\
\hline Thorium-230 & $0 / 40$ & - & - & - & - & - \\
\hline Thorium-232 & $1 / 40$ & 77,700 & 77,700 & 77,700 & 77,700 & - \\
\hline Tritium & $15 / 40$ & 1.76 & 33.9 & 6.37 & 3.33 & 8.10 \\
\hline Uranium-233/234 & $1 / 40$ & 3.61 & 3.61 & 3.61 & 3.61 & - \\
\hline Uranium-235/236 & $2 / 40$ & 1.71 & 9.85 & 5.78 & 5.78 & 5.76 \\
\hline Uranium-238 & & & & & & \\
\hline
\end{tabular}

${ }^{a}$ Values rounded to three significant digits

\subsection{SMEAR MEASUREMENT AND ANALYTICAL RESULTS}

Radiation measurement data summarized in Sect. 3.2 and laboratory analytical data summarized in Sect. 3.3 are combined here for a high-level analysis of project results. The objective is to identify noteworthy trends and to state general observations based on a cursory review of investigation results. It is presumed that ORNL S\&M Project representatives will perform a more rigorous analysis in their attempt to develop screening criteria for waste acceptance at EMWMF. 
The plots presented in Appendix D are divided into two primary groups: 1) results associated with reactor facilities 3001, 3010, and 3042; and 2) results associated with non-reactor facilities 3028, 3029, 3038, 3517, and 4507. This grouping was used assuming reactor facilities would most likely contain a similar contaminant mixture (i.e., long-lived fission products), while non-reactor facilities could produce a broad and varying range of contaminants. Plots use colors and symbols to distinguish between facilities within the group.

Within each group, data were divided into subgroups based on the type of radiation (alpha or beta) that is most commonly associated with each radionuclide. In some cases, a radionuclide is associated with both alpha and beta radiation, and is therefore, included in both subgroups. For example, Cm242 is included only in the alpha radiation subgroup, Cs-137 is included only in the beta radiation subgroup, but U-238 is included in both subgroups because it is an alpha emitter that produces short-lived beta emitting decay products.

Each App. D plot shows net alpha or beta cpm on the x-axis and laboratory-reported concentration in $\mathrm{pCi} /$ sample on the $\mathrm{y}$-axis. The cpm values are as measured using UCOR instruments. Only detected $\mathrm{pCi}$ /sample results are included in plots, thus plots for rarely-detected analytes like tritium and I-129 will be sparsely populated or omitted. A non-detected is defined here as a laboratory analytical result flagged by the SMO validator as "U” (non-detected) or "UJ" (non-detected and estimated). All plots are presented using a log-log scale given the large range of both cpm and $\mathrm{pCi} /$ sample values in the dataset. As in the App. C summary, plots for Am-241 include results reported using the alpha spectrometric analytical method.

For the reactor group, the Am-241 is the most notable alpha emitter and Cs-137 is the most notable beta emitter. Figure 3.3 presents the plot of pCi/sample of Am-241 versus cpm alpha. The plot suggests a linear relationship between Am-241 and alpha count rate in Bldg. 3042, though Am-241 was not detected in Bldg. 3010 samples. Figure 3.4 presents a plot of pCi/sample of Cs-137 versus cpm beta. The plot suggests a linear relationship in all three reactor buildings, most obviously in Bldg. 3042. The two detected results for Bldg. 3010 fall well outside the Bldg. 3001 and 30142 populations, suggesting the beta response is due in large part to other removable contaminants. The App. C data summary indeed shows that other beta emitters are present on Bldg. 3010 smears, most notably Co-60 and Tc-99. 
For the non-reactor group, the Cm-243/234 is the most notable alpha emitter and Cs-137 is the most notable beta emitter. Figure 3.5 presents the plot of pCi/sample of Cm-243/234 versus cpm alpha. The plot suggests a linear relationship between $\mathrm{Cm}-243 / 234$ and alpha count rate in Bldgs. 3028 and 3029, though Cm-243/234 was not detected in Bldg. 3038 samples. Figure 3.6 presents a plot of $\mathrm{pCi} /$ sample of Cs-137 versus cpm beta. The plot suggests a linear relationship in all non-reactor buildings, though the scale of activity varies by facility.

This combined analysis of radiation measurement and laboratory analytical data is supplemented by the process knowledge reviews conducted by ORAU investigators. These reviews identified existing facility documentation that adequately described the facilities' physical descriptions, operational histories, list of likely contaminants, and other information that future investigators could use to plan waste disposition activities. Key reference documents include the following:

- An Account of Oak Ridge National Laboratory's Thirteen Nuclear Reactors (ORNL 2009) for Bldgs. 3001, 3005, 3010, and 3042;

- Oak Ridge National Laboratory - Site Description (ORAU 2006) for all subject facilities;

- Preliminary Hazard Screening for Building 3001, Oak Ridge Graphite Reactor, and Support Buildings (3002, 3003, and 3018) Oak Ridge National Laboratory, Oak Ridge Tennessee (UCOR 2013a);

- Preliminary Hazard Screening for Miscellaneous Areas and Activities at ORNL, Oak Ridge, Tennessee, Rev. 7 and Rev. 10 (UCOR 2011a and 2013b) for Bldg. 3005;

- Preliminary Hazard Screening for Buildings 3010, 3009, \& 3119 Oak Ridge National Laboratory, Oak. Ridge, Tennessee (UCOR 2013c) for Bldg. 3010;

- Hazard Assessment Document for Building 3028, Oak Ridge National Laboratory, Oak Ridge, Tennessee (UCOR 2012) for Bldg. 3028;

- Preliminary Hazard Screening for the Source Development Laboratory (UCOR 2011b) for Bldg. 3029;

- Documented Safety Analysis for Building 3038 Isotope Development Laboratory Oak Ridge National Laboratory Oak Ridge, Tennessee (BJC 2005) for Bldg. 3038; 
- Hazards Assessment Document for the Oak Ridge Research Reactor, Building 3042, Oak Ridge National Laboratory, Oak Ridge, Tennessee (UCOR 2013d) for Bldg. 3042;

- Documented Safety Analysis for Building 3517, Fission Product Development Laboratory, Oak Ridge, Tennessee (UCOR 2013e) for Bldg. 3517;

- Hazard Assessment Document for Buildings 4507, High Radiation Level Chemical Development Facility, Oak Ridge National Laboratory, Oak Ridge, Tennessee, (UCOR 2013f) for Bldg. 3517; and

- Hazard Assessment Document for the Homogeneous Reactor Experiment, Bldg. 7500 at the Oak Ridge National Laboratory, Oak Ridge, Tennessee (UCOR 2013g) for Bldg. 7500.

Due to time and access constraints, ORAU investigators were unable to produce detailed lists of hazardous items and substances such as potential polychlorinated biphenyl reservoirs, asbestos containing materials, etc. ORAU investigators were able to interview some current and former site personnel with facility-specific historical knowledge. These "living memory" summaries are presented in App. E of this report. Investigators also prepared summaries of the types and extent of chemical contamination and hazardous materials of concern that were observed during a recent tour of the subject facilities. These summaries are presented in App. F of this report. 


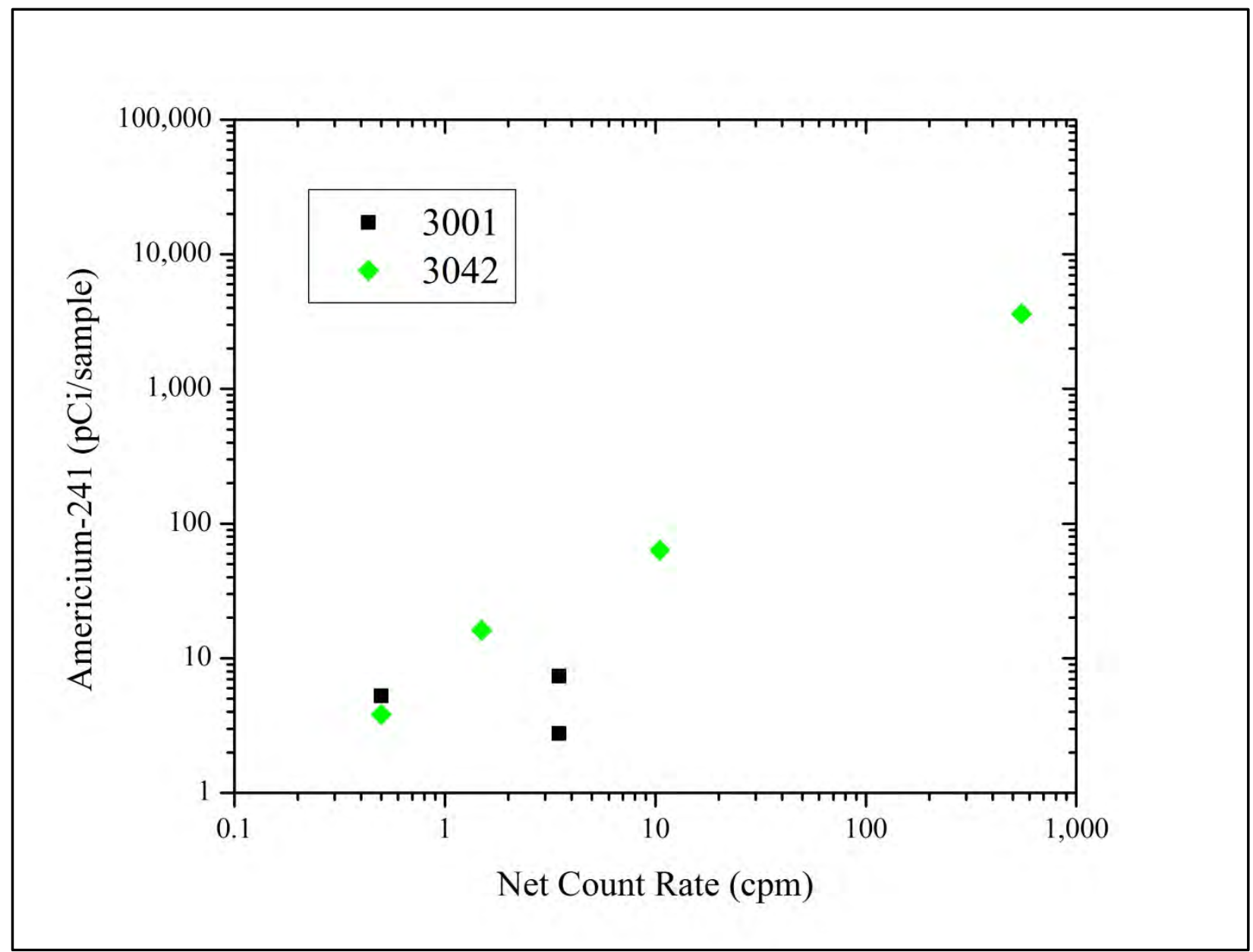

Fig. 3.3. Alpha cpm vs. pCi/sample of Am-241 in the Reactor Building Group 


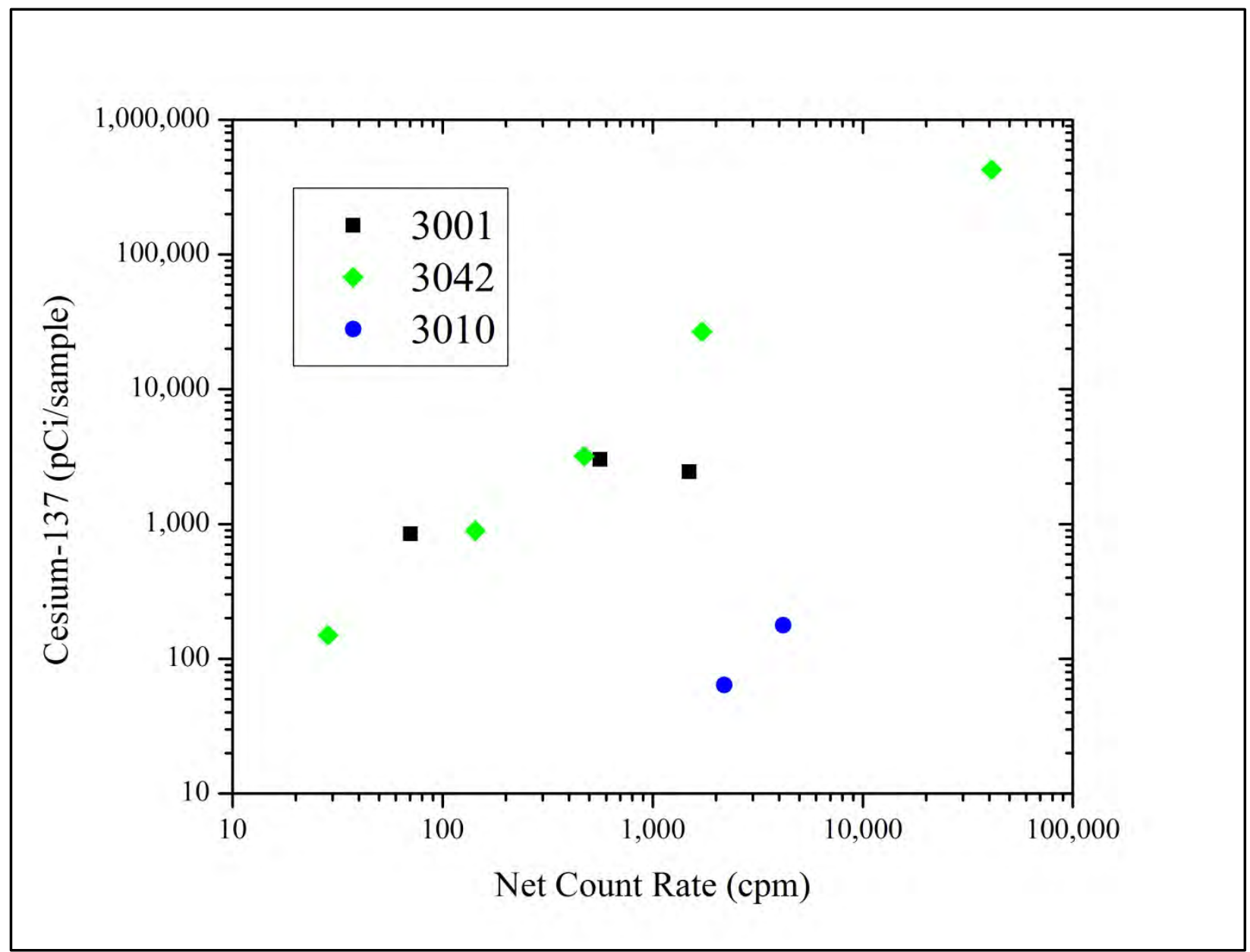

Fig. 3.4. Beta cpm vs. pCi/sample of $\mathrm{Cs}-137$ in the Reactor Building Group 


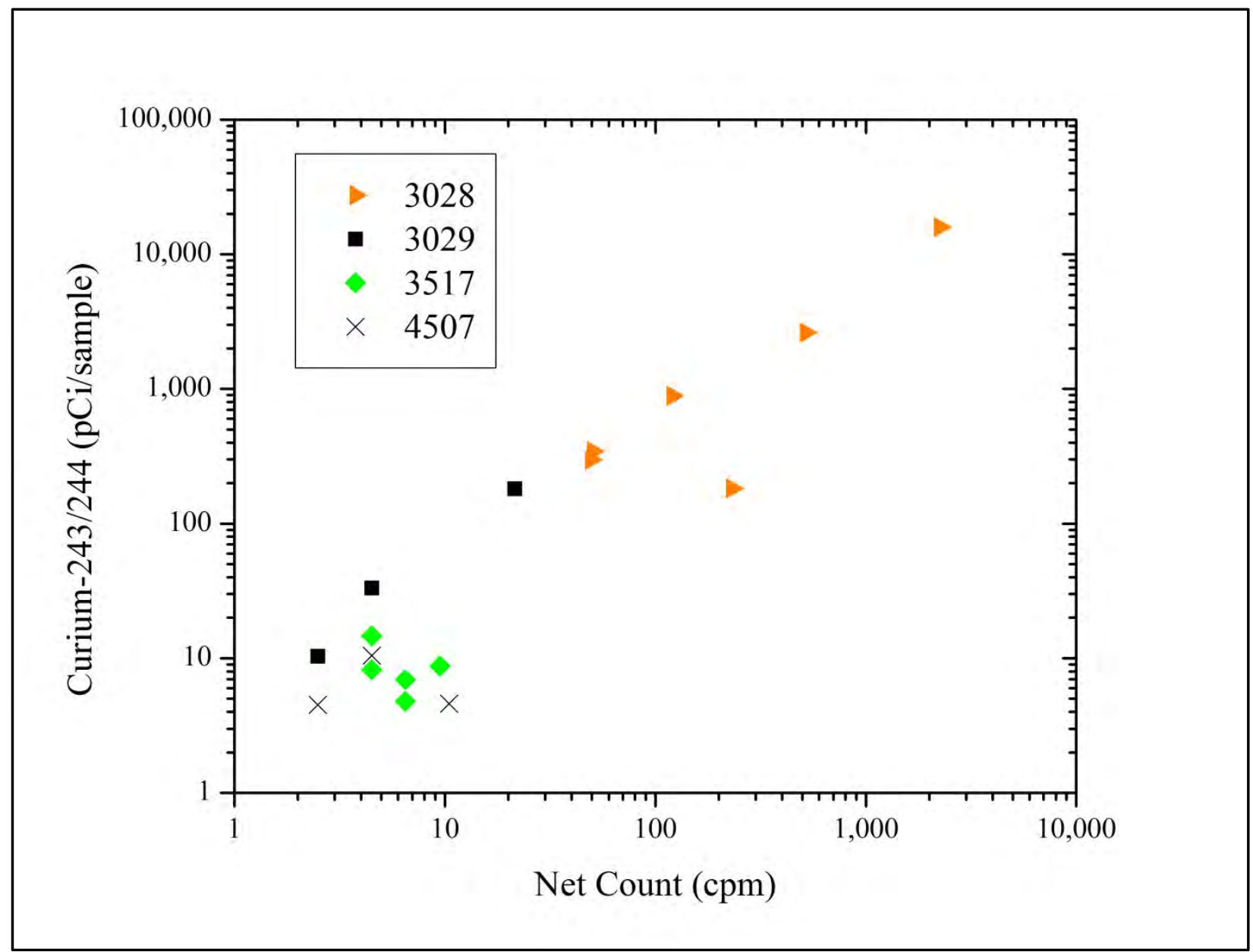

Fig. 3.5. Alpha cpm vs. pCi/sample of $\mathrm{Cm}-243 / 244$ in the Non-reactor Building Group 


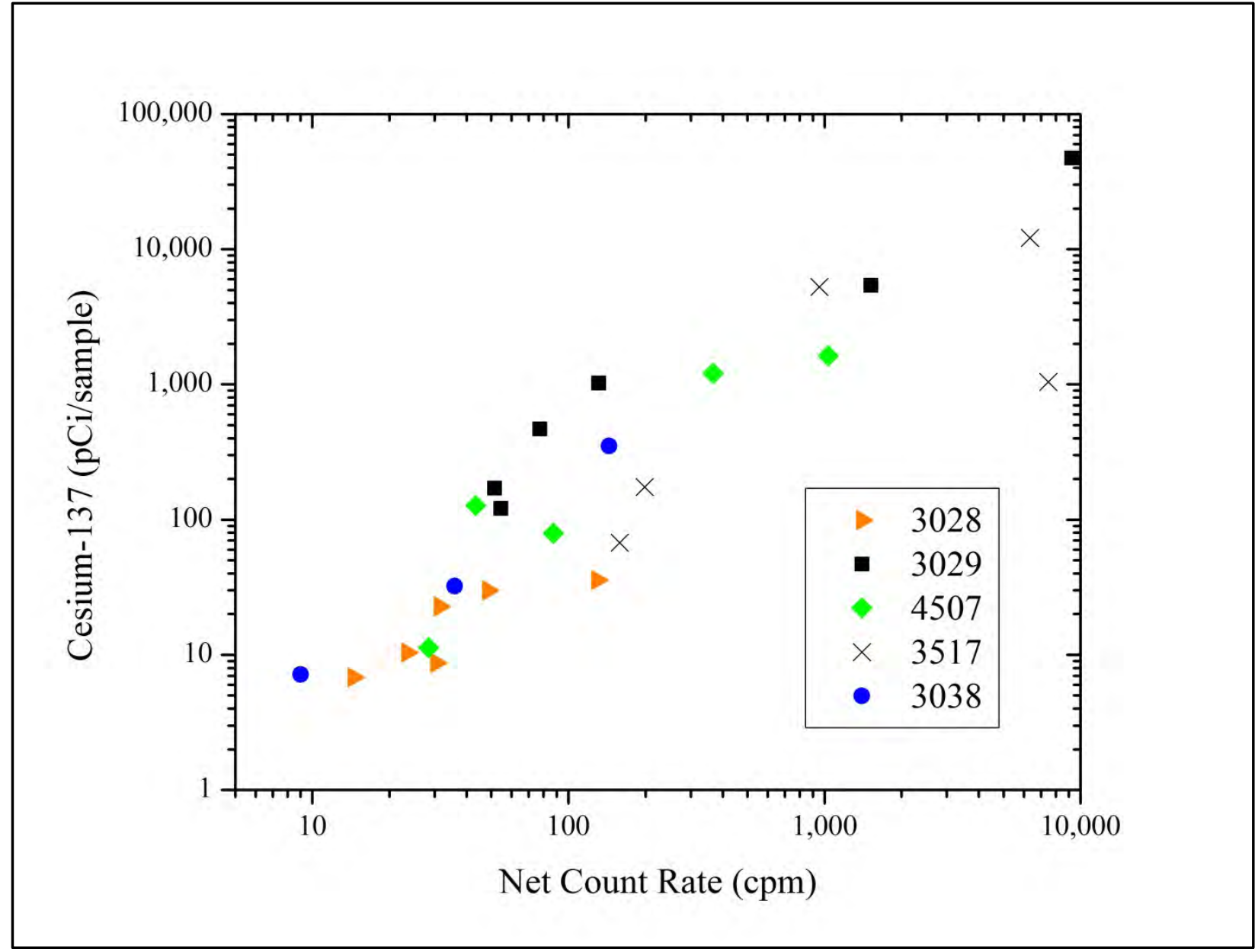

Fig. 3.6. Beta cpm vs. pCi/sample of Cs-137 in the Non-reactor Building Group

\section{SUMMARY AND CONCLUSION}

As requested by DOE, ORAU collected and analyzed removable activity levels across accessible ORNL S\&M Project facilities. Seventy-two masselin LAWs were collected by ORAU investigators from seven facilities (Bldgs. 3001, 3010, 3028, 3029, 3042, 3517, and 4507), and ORNL S\&M Project personnel provided an additional 100 standard paper smears for one inaccessible facility (Bldg. 3038). Two of the target facilities (Bldgs. 3005 and 7500) were inaccessible during the fieldwork phase of the project, and the ORNL S\&M Project were unable to provide smears within the same window of opportunity; therefore, no smear data are available for these facilities. 
Alpha and beta radiation levels were measured on available smears and 40 samples were ultimately selected by ORAU and ORNL S\&M project personnel for submittal to a SMO laboratory. The analytical laboratory analyzed the samples for 24 radiological analytes, and the SMO validated laboratory data packages. No data were rejected.

A review of alpha and beta measurement data show ORAU and UCOR instruments produced consistent cpm responses, and smears successfully represented a wide range of contamination levels across facilities. The analytical data also show a wide range of activity levels and a mixture of facility-specific isotopic constituents. Most notable among these constituents is the fission product Cs-137, which appears to produce a linear relationship between detector response (beta) and removable concentration.

This report presents the methods for collecting the data and radiation measurement results for all LAWs or standard paper smear composites (for Bldg. 3038). Laboratory analytical data summarized herein have been provided to ORNL S\&M Project personnel and are available in PEMS under project name ORNL ISO. ORNL S\&M project personnel may use these data to develop facilityspecific screening criteria for waste disposition planning, assuming stakeholder approvals are obtained. ORAU's objective was to provide the data within the approved budgetary and temporal boundary conditions. This report concludes ORAU's scope. The ORNL S\&M Project or future planners may use this data and process knowledge to develop screening criteria, if necessary. 


\section{REFERENCES}

BJC 2005. Documented Safety Analysis for Building 3038 Isotope Development Laboratory Oak Ridge National Laboratory Oak Ridge, Tennessee, DSA-OR-3038-0011, Rev. 8, December.

EPA 2006. Guidance on Systematic Planning Using the Data Quality Objectives Process. EPA QA/G-4, EPA/240/B-06/001. U.S. Environmental Protection Agency. Washington, D.C.

DOE 2012. Waste Handling Plan for Surveillance and Maintenance Activities at the Oak Ridge National Laboratory, Oak Ridge, Tennessee. DOE/OR/01-2565\&D2. U.S. Department of Energy, Office of Environmental Management. Oak Ridge, Tennessee.

DOE 2013. Sampling and Analysis Plan for Analyzing Removable Activity in Various Oak Ridge National Laboratory Surveillance and Maintenance Project Facilities, Oak. Ridge, Tennessee, DOE/OR/01-2626\&D1, Office of Environmental Management, Oak Ridge, Tennessee, August.

ORAU 2006. Oak Ridge National Laboratory - Site Description, ORAUT-TKBS-0012-2 Rev. 01, prepared by the National Institute for Occupational Safety and Health Dose Reconstruction Project Team, August.

ORNL 2009. An Account of Oak Ridge National Laboratory's Thirteen Nuclear Reactors, ORNL/TM2009/181, prepared by Murray W. Rosenthal, August.

UCOR 2011b. Preliminary Hazard Screening for the Source Development Laboratory, PHS-OR-3029-1005, prepared for the Department of Energy Office of Environmental Management, Oak Ridge, Tennessee.

UCOR 2011a. Preliminary Hazard Screening for Miscellaneous Areas and Activities at ORNL, Oak Ridge, Tennessee, PHS-OR-S\&M-1000, Rev. 7, prepared for the Department of Energy Office of Environmental Management, Oak Ridge, Tennessee.

UCOR 2012. Hazard Assessment Document for Building 3028, Oak Ridge National Laboratory, Oak Ridge, Tennessee, HAD-OR-3028-0058, Rev. 3, April 2012, prepared for the Department of Energy Office of Environmental Management, Oak Ridge, Tennessee.

UCOR 2013a, Preliminary Hazard Screening for Building 3001, Oak Ridge Graphite Reactor, and Support Buildings (3002, 3003, and 3018) Oak Ridge National Laboratory, Oak Ridge Tennessee, PHS-OR-OGR0998/Rev. 2, prepared for the Department of Energy Office of Environmental Management, Oak Ridge, Tennessee, July.

UCOR 2013b. Preliminary Hazard Screening for Miscellaneous Areas and Activities at ORNL, Oak Ridge, Tennessee, PHS-OR-S\&M-1000, Rev. 10, prepared for U.S. Department of Energy Office of Environmental Management, Oak Ridge, Tennessee.

UCOR 2013c. Preliminary Hazard Screening for Buildings 3010, 3009, \& 3119 Oak Ridge National Laboratory, Oak Ridge, Tennessee, PHS-OR-3010-0840 Rev.4, prepared for U.S. Department of Energy Office of Environmental Management, Oak Ridge, Tennessee.

UCOR 2013d. Hazards Assessment Document for the Oak Ridge Research Reactor, Building 3042, Oak Ridge 
National Laboratory, Oak Ridge, Tennessee, HAD-OR-3042-0030, Rev. 10, prepared for the U.S. Department of Energy Office of Environmental Management, Oak Ridge, Tennessee, May.

UCOR 2013e. Documented Safety Analysis for Building 3517, Fission Product Development Laboratory, Oak Ridge, Tennessee, DSA-OR-3517-0013, Rev. 12, prepared for U.S. Department of Energy Office of Environmental Management, Oak Ridge, Tennessee.

UCOR 2013f. Hazard Assessment Document for Buildings 4507, High Radiation Level Chemical Development Facility, Oak Ridge National Laboratory, Oak Ridge, Tennessee, HAD-OR-4507-0059 Rev. 4, prepared for the U.S. Department of Energy Office of Environmental Management, Oak Ridge, Tennessee, January.

UCOR 2013g. Hazard Assessment Document for the Homogeneous Reactor Experiment, Bldg. 7500 at the Oak Ridge National Laboratory, Oak Ridge, Tennessee, HAD-OR-HRE-0019, Rev. 7, prepared for the U.S. Department of Energy Office of Environmental Management, Oak Ridge, Tennessee, May. 
APPENDIX A

FACILITY MAPS SHOWING SMEAR LOCATIONS 


\section{Building 3001}

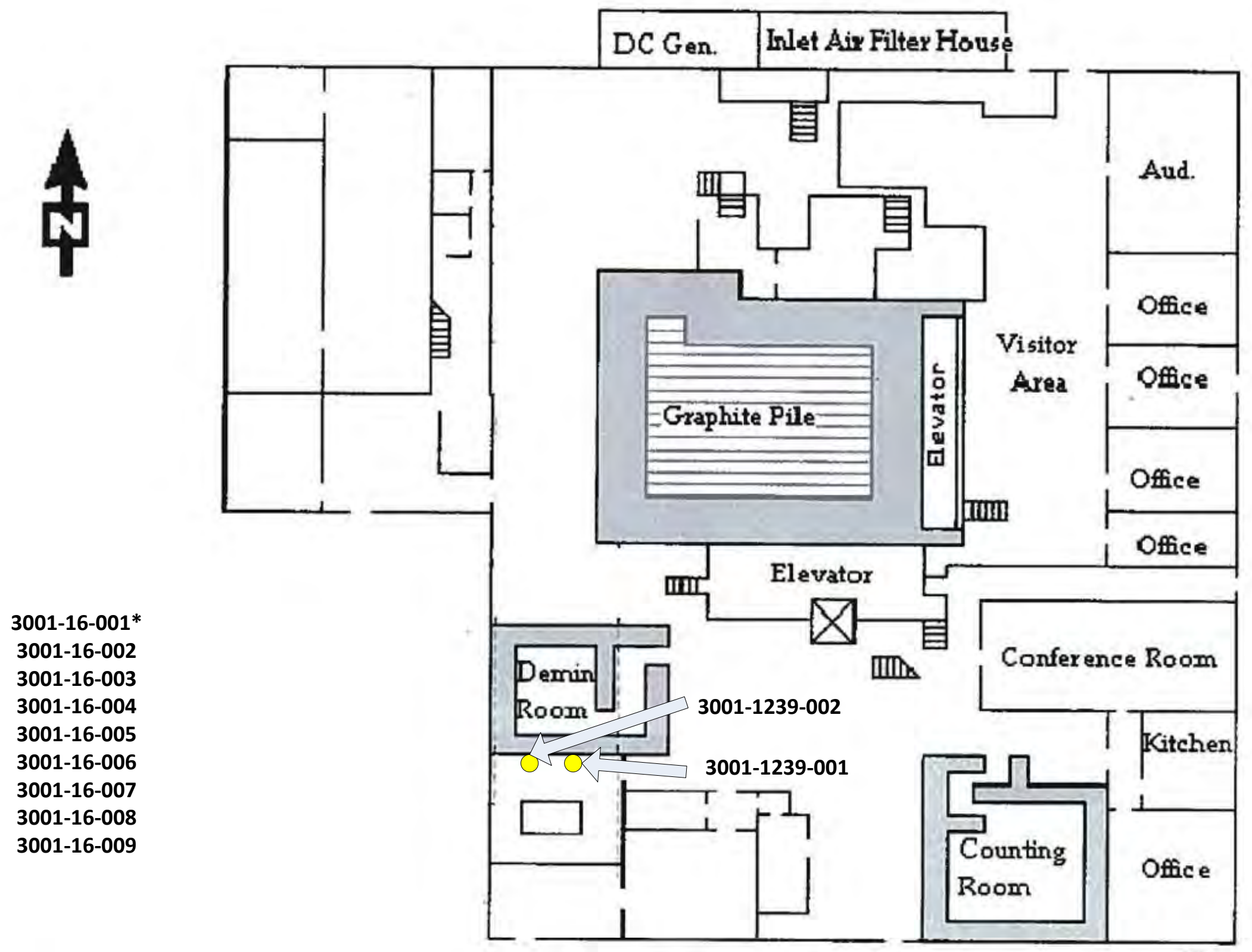

Samples Taken in Room 110A

*The locations of samples listed to the left of the figure could not be illustrated due to classification restrictions 
Building 3010
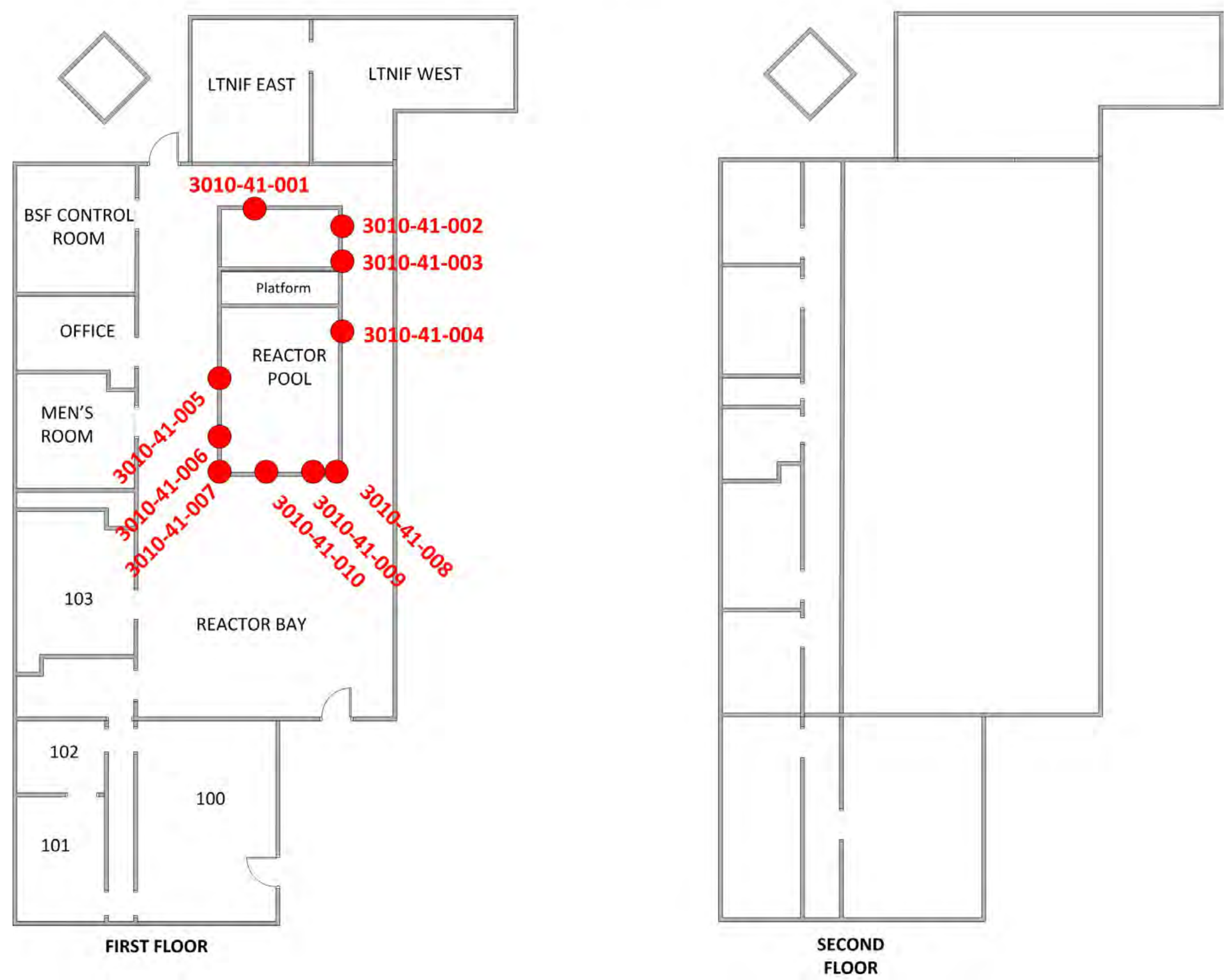


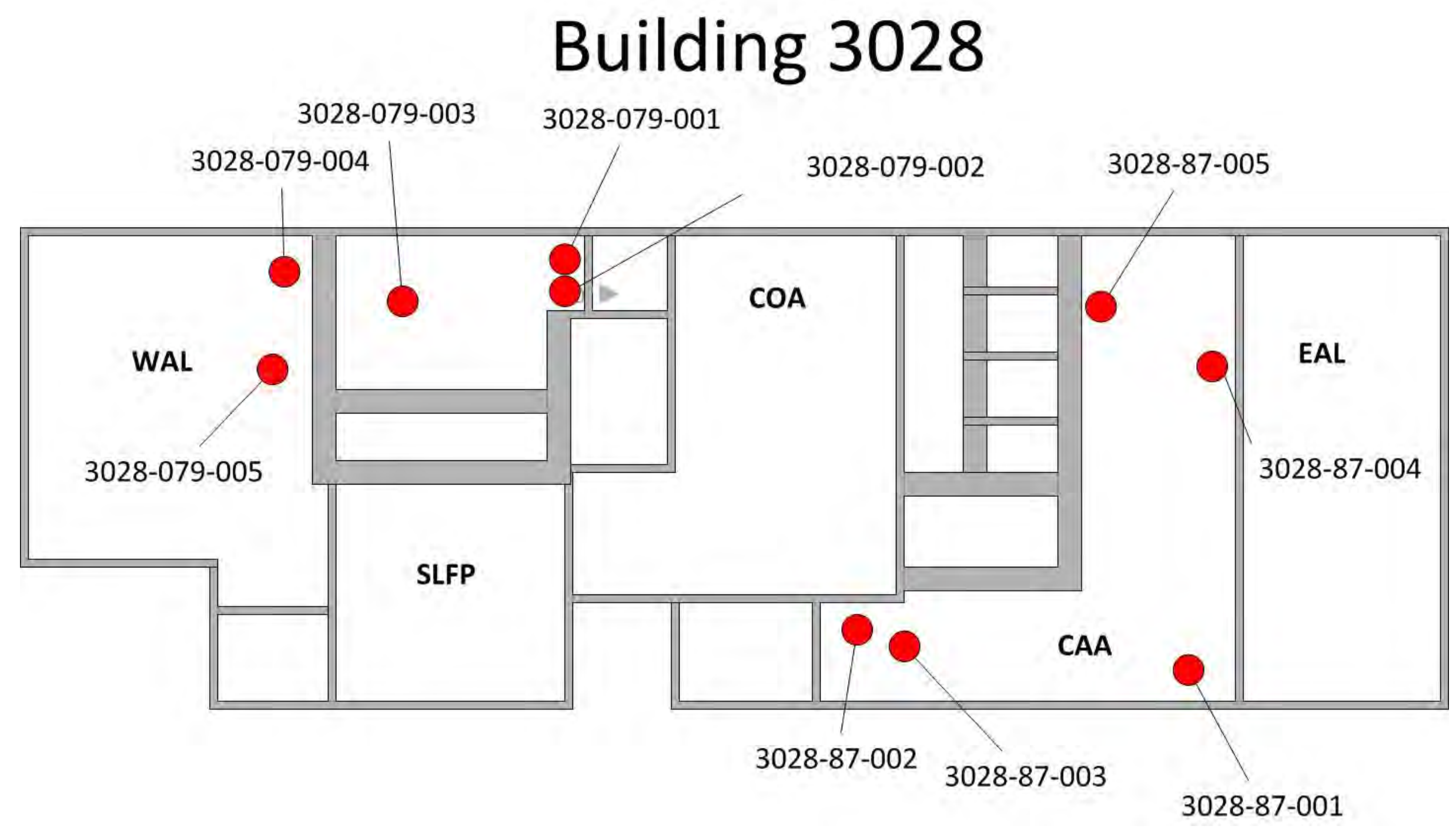




\section{Building 3029}

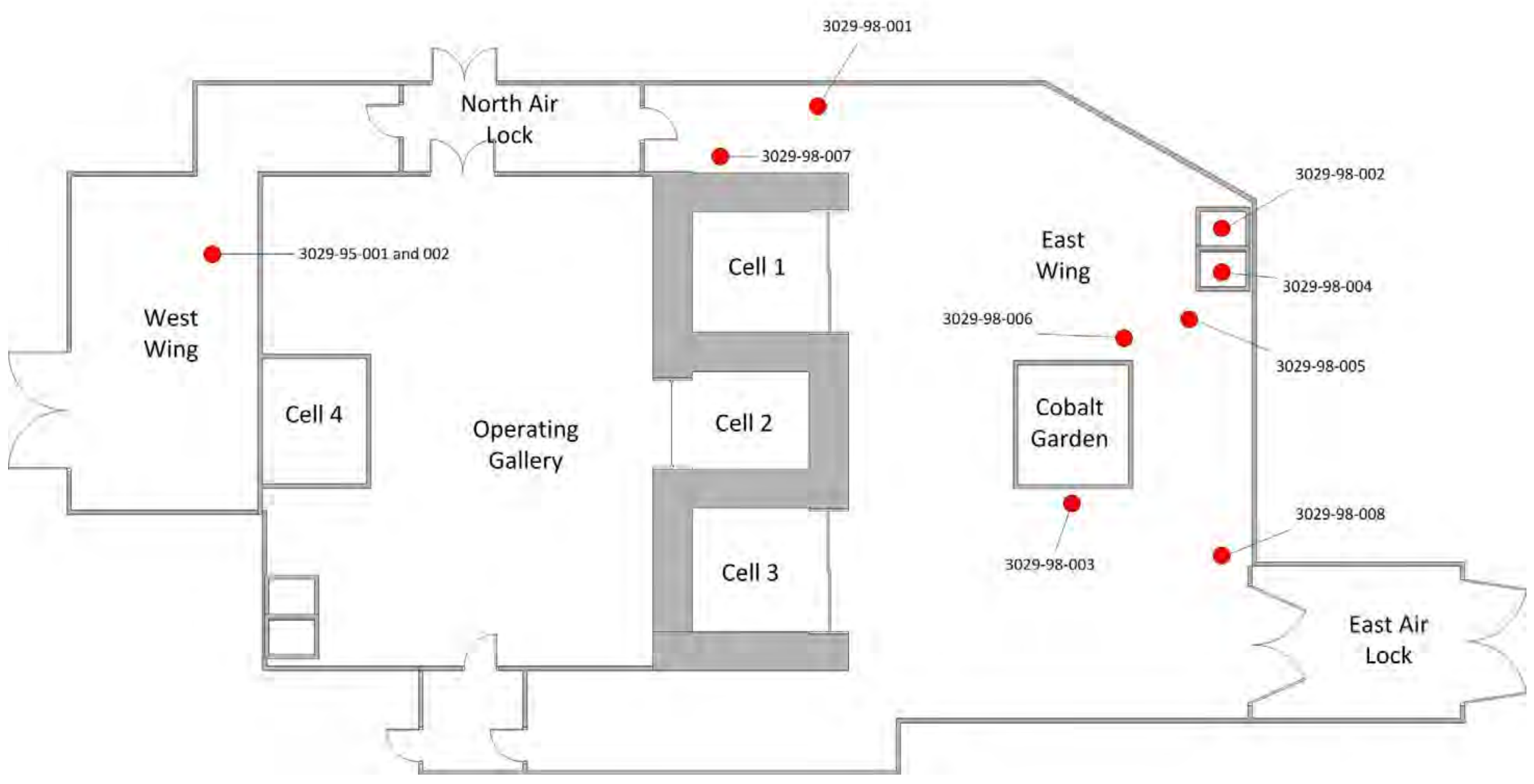




\section{Building 3038}

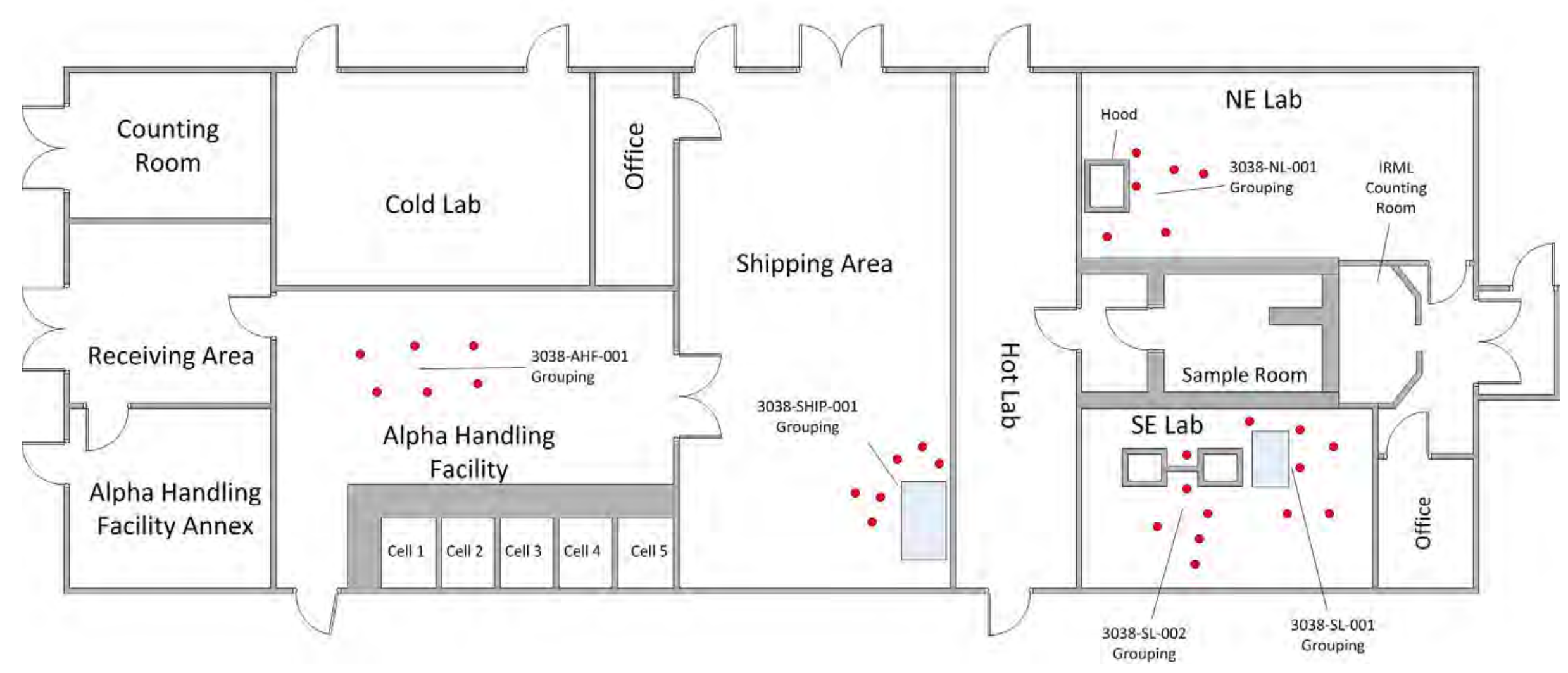


BLDG 3517

FIRST FLOOR

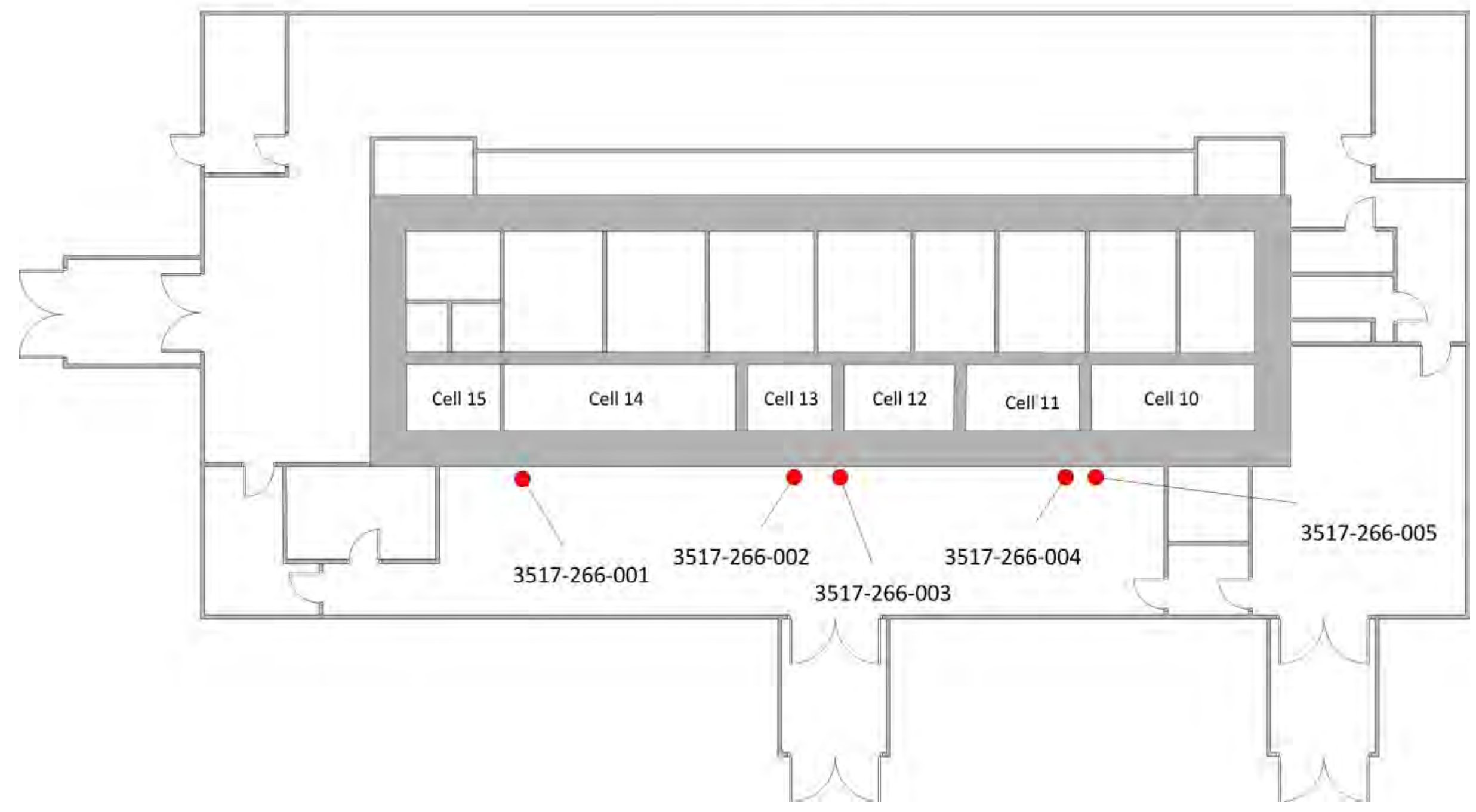


BLDG 3517

SECOND FLOOR

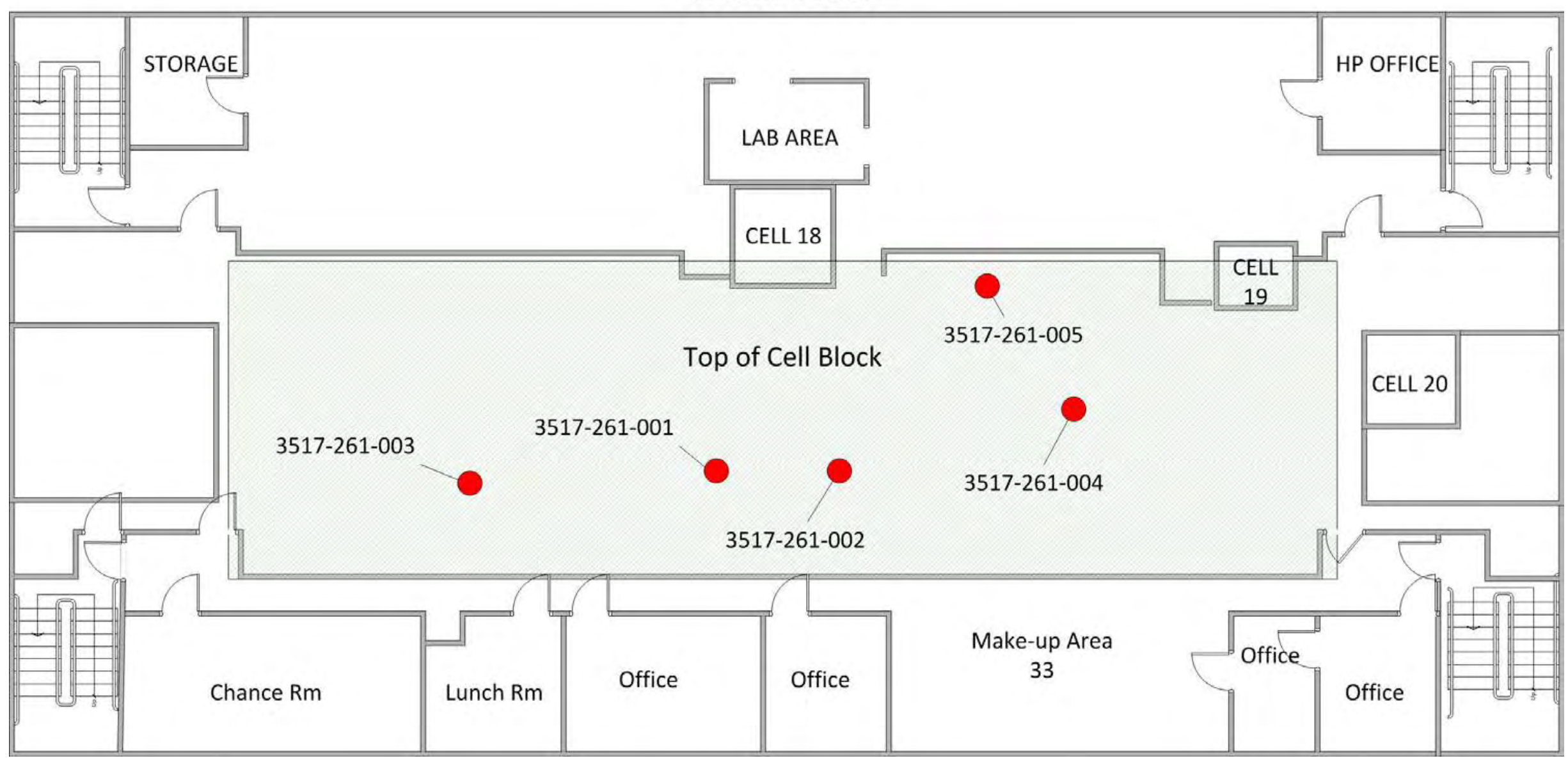




\section{Building 4507}

First Floor

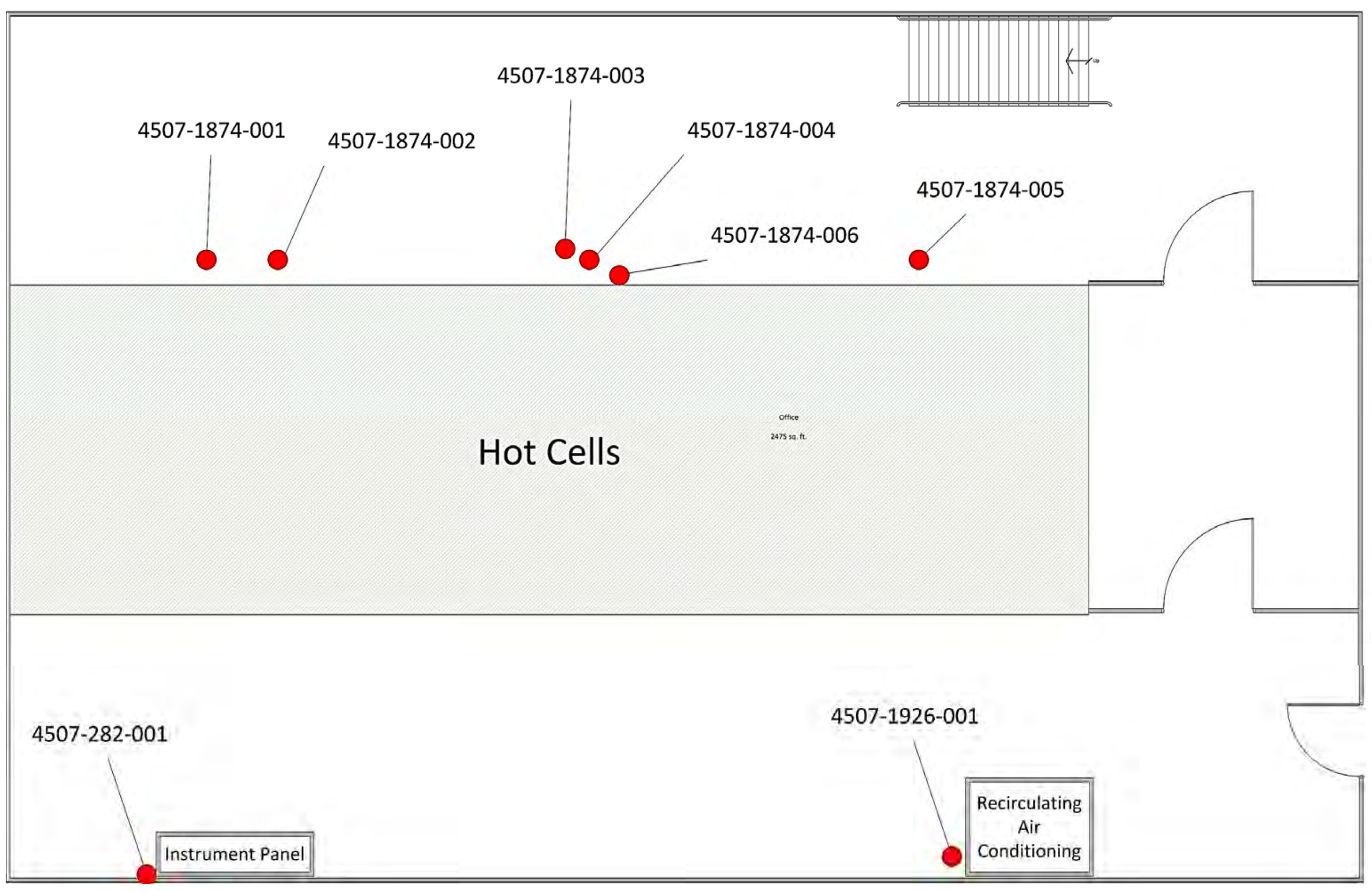




\section{Building 4507}

Third Floor

Penthouse

(N)

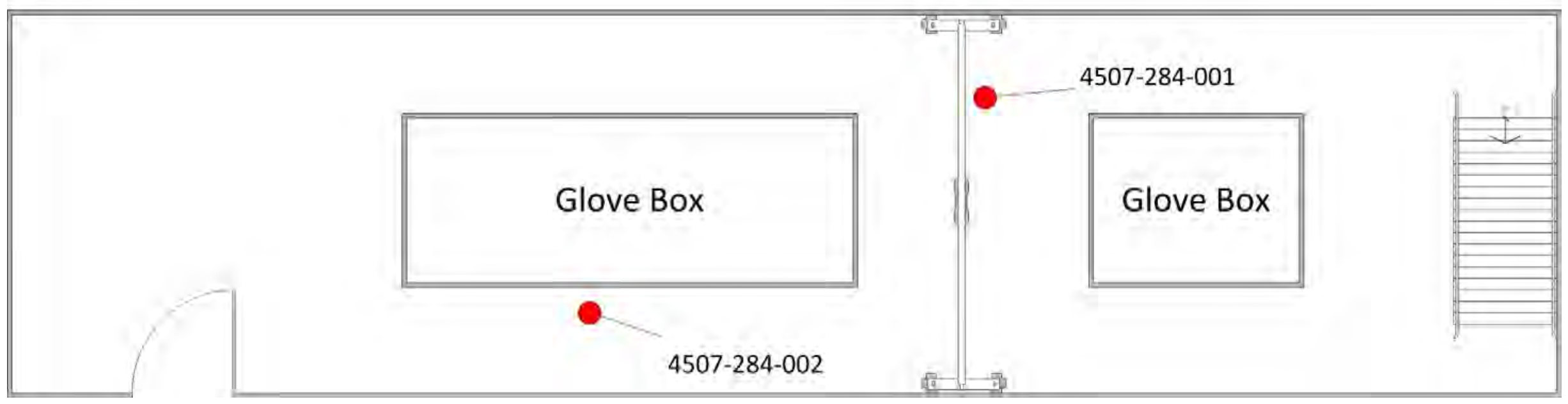


APPENDIX B

PHOTOGRAPHS OF SMEAR LOCATIONS 


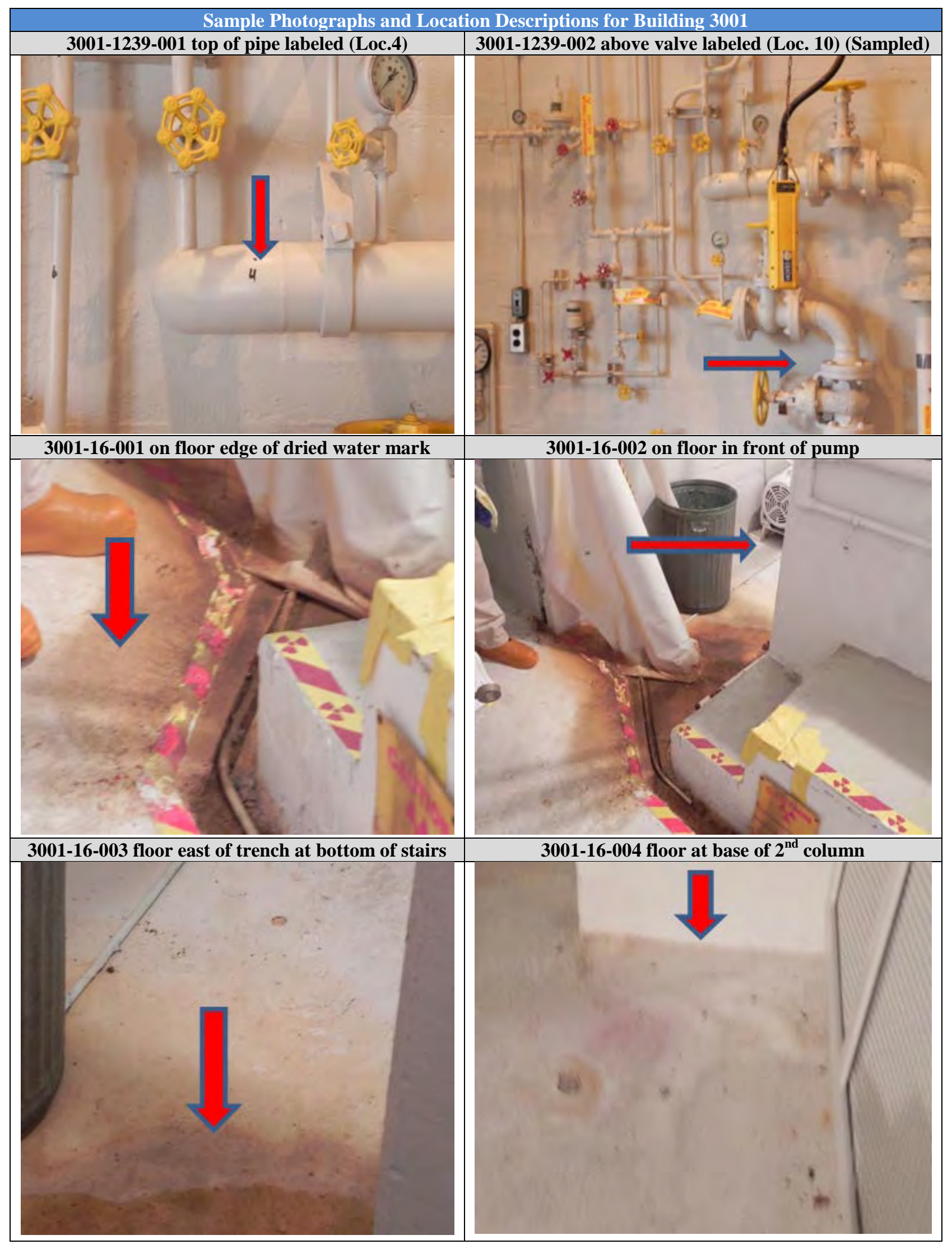




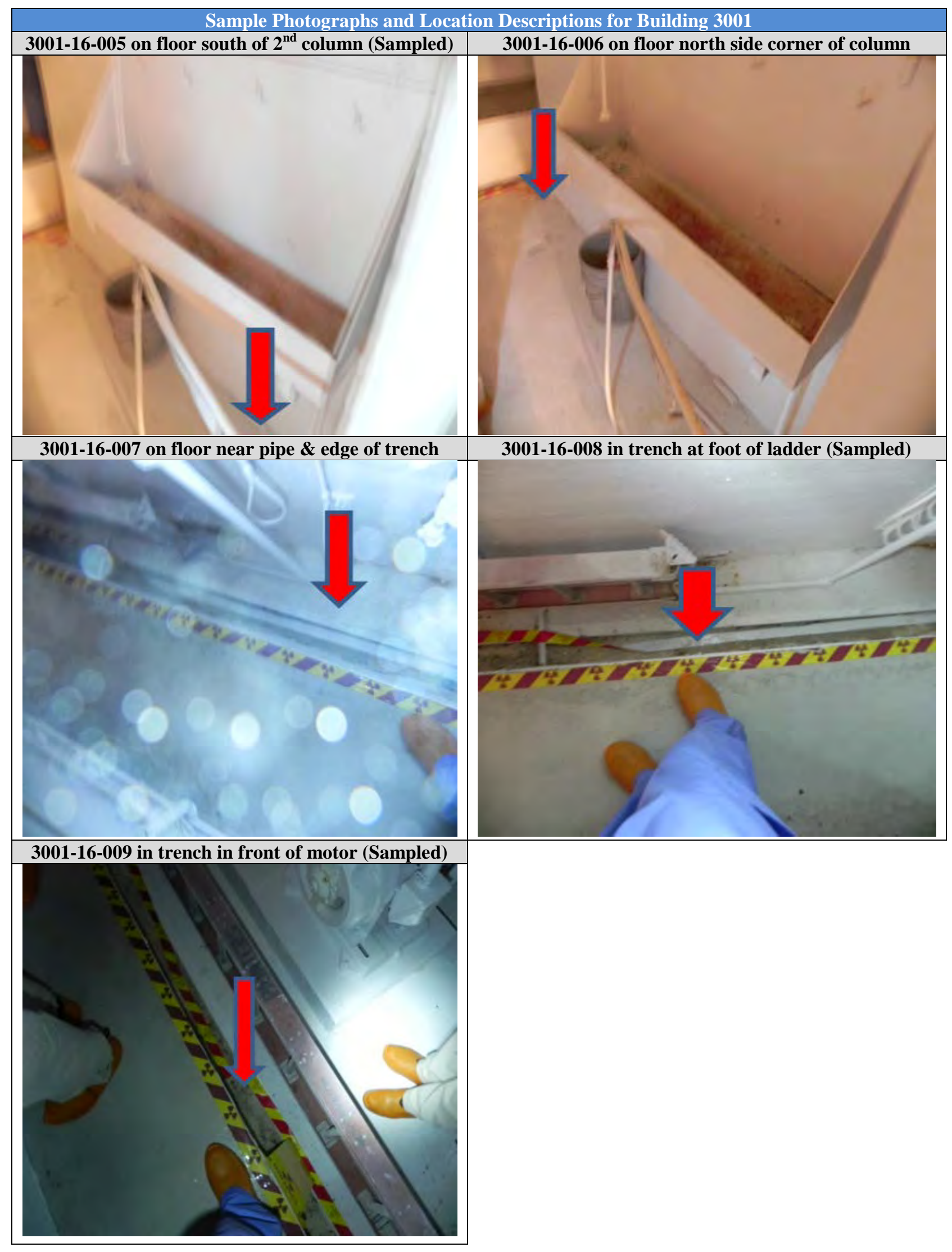




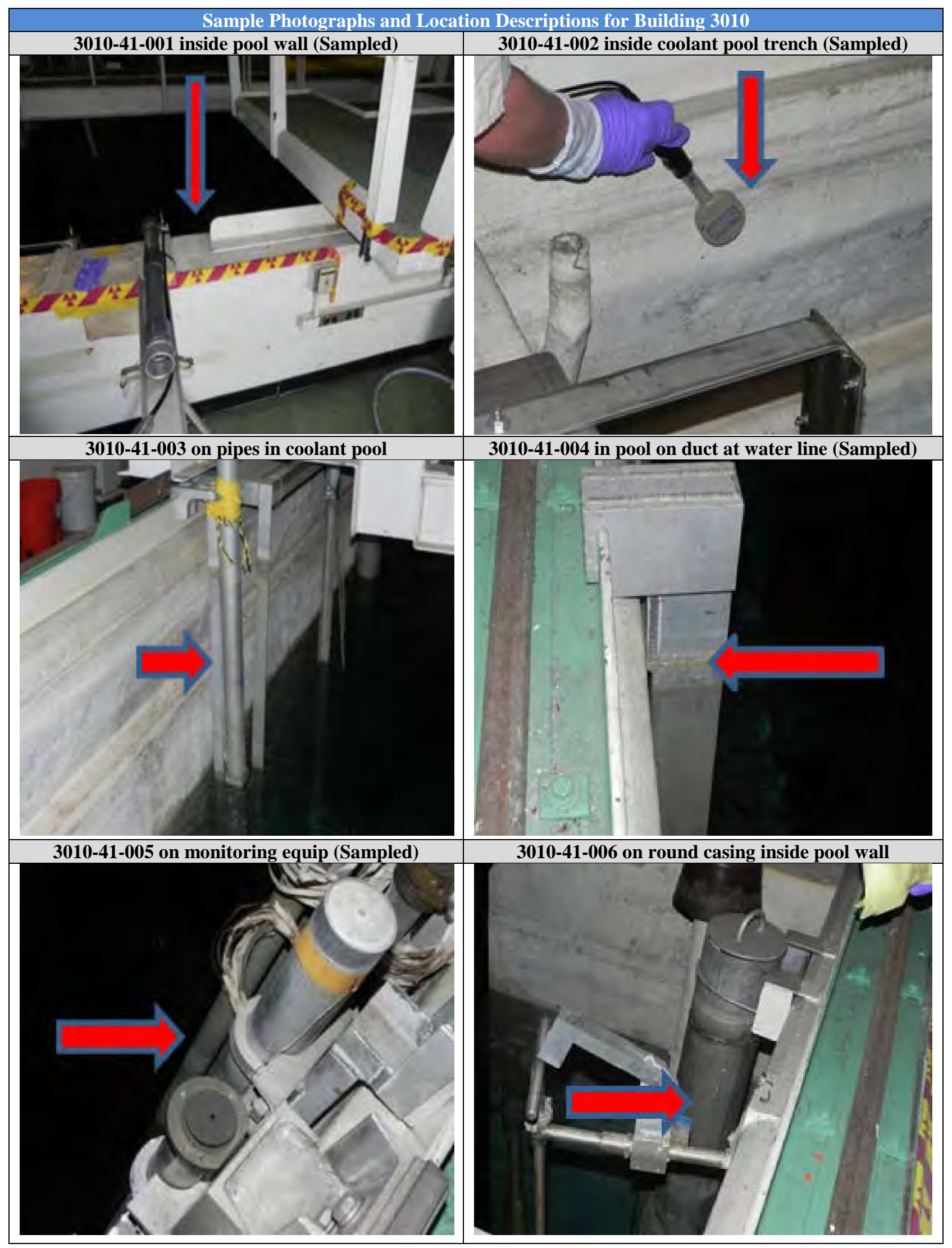




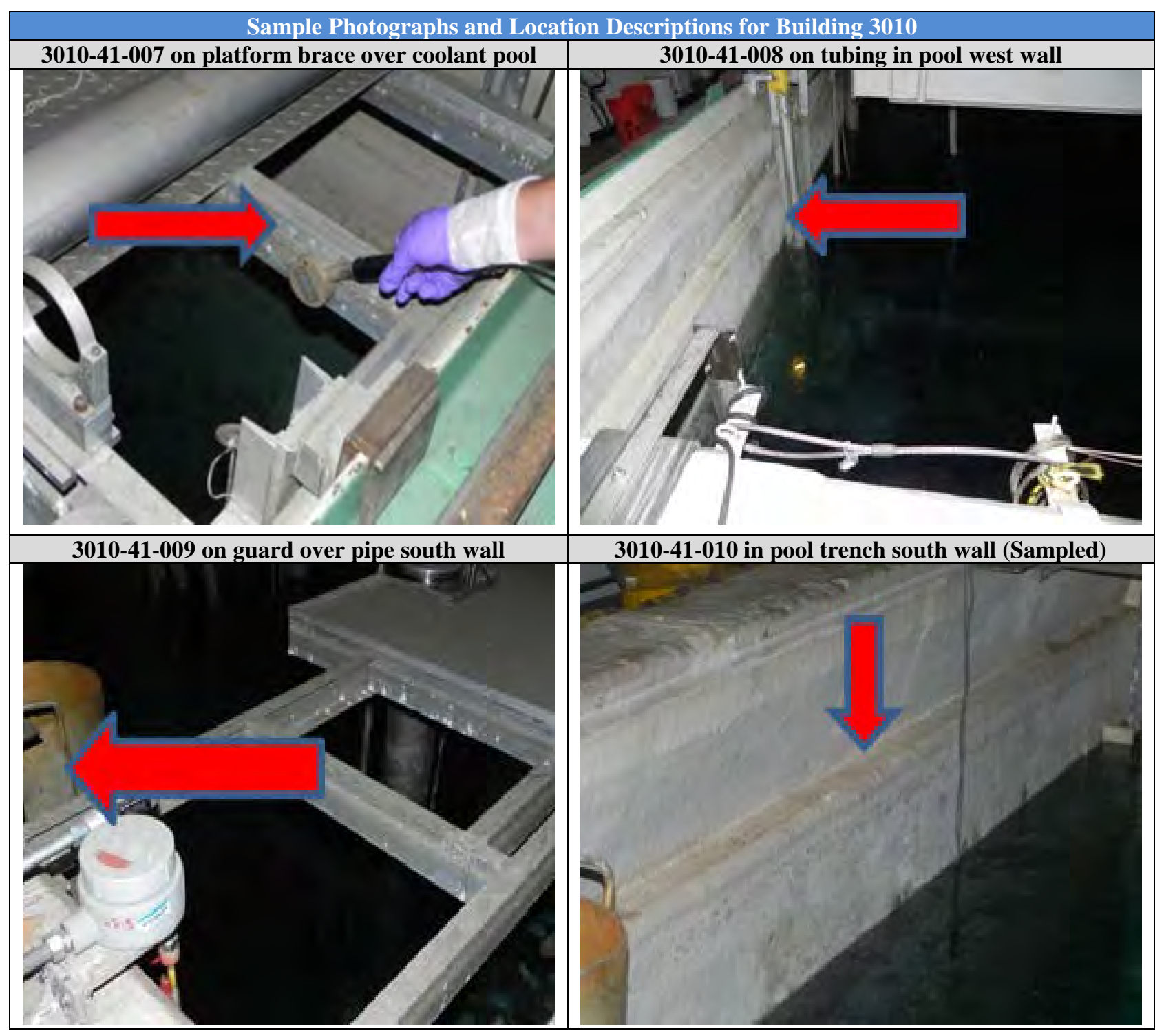




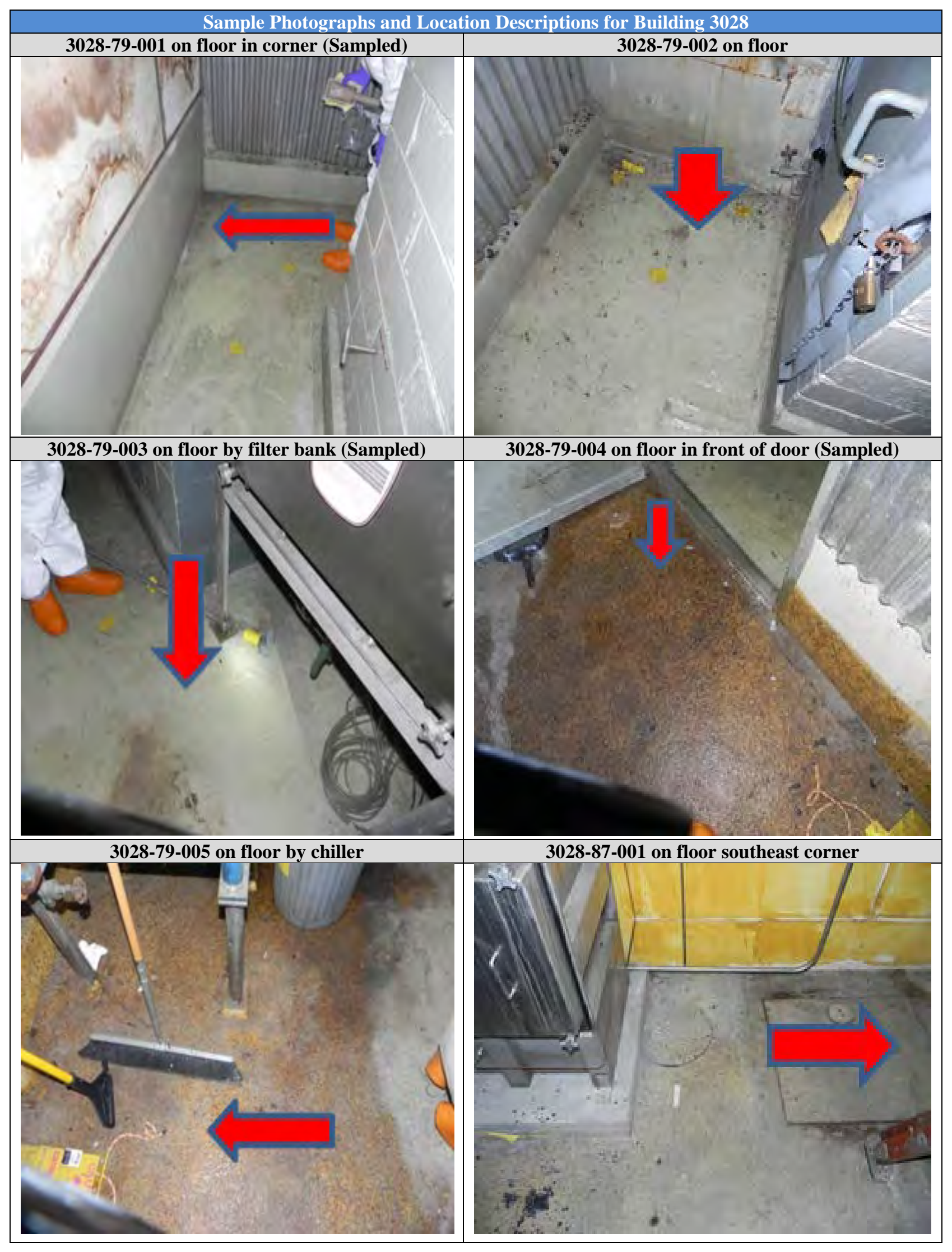




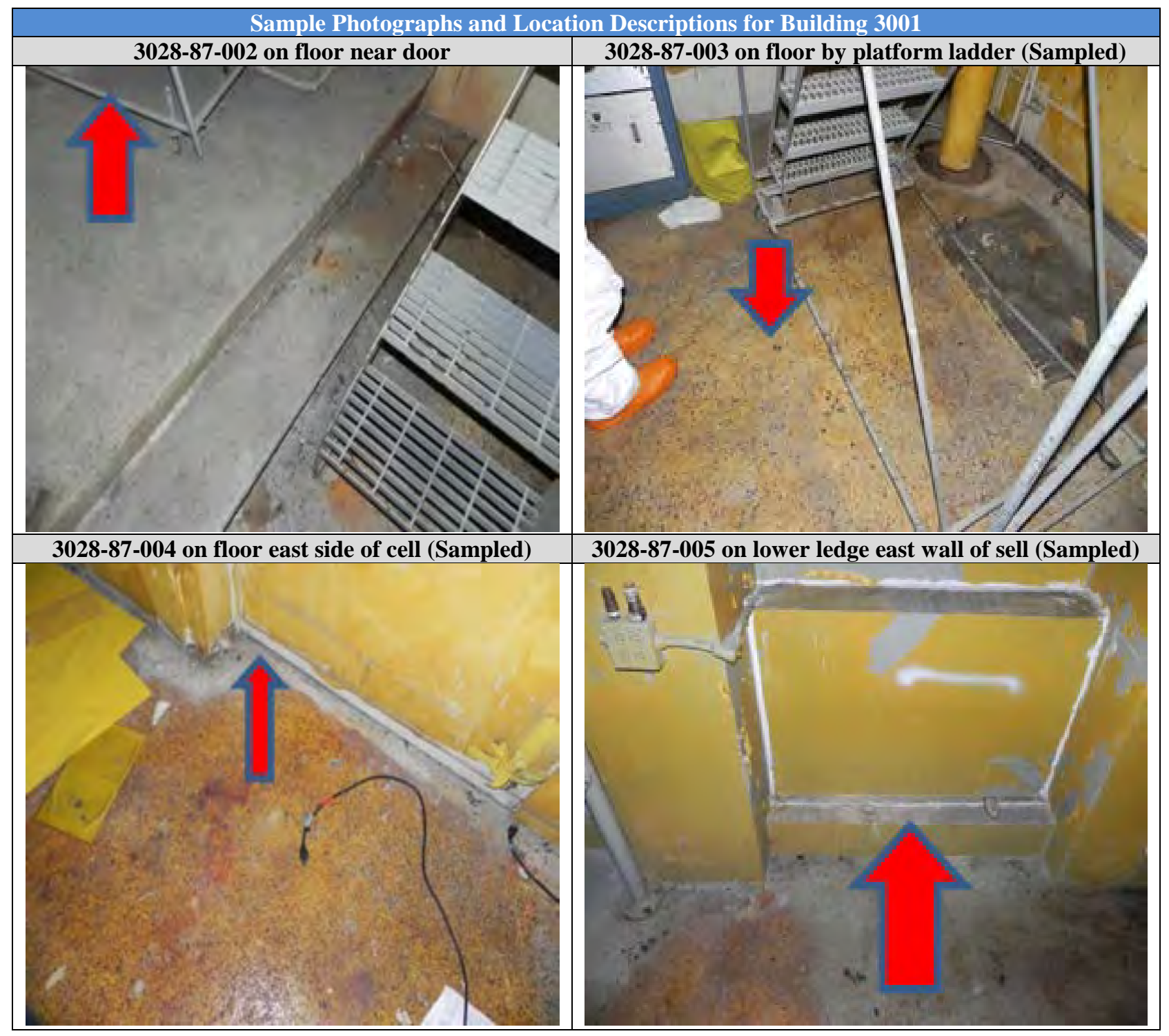




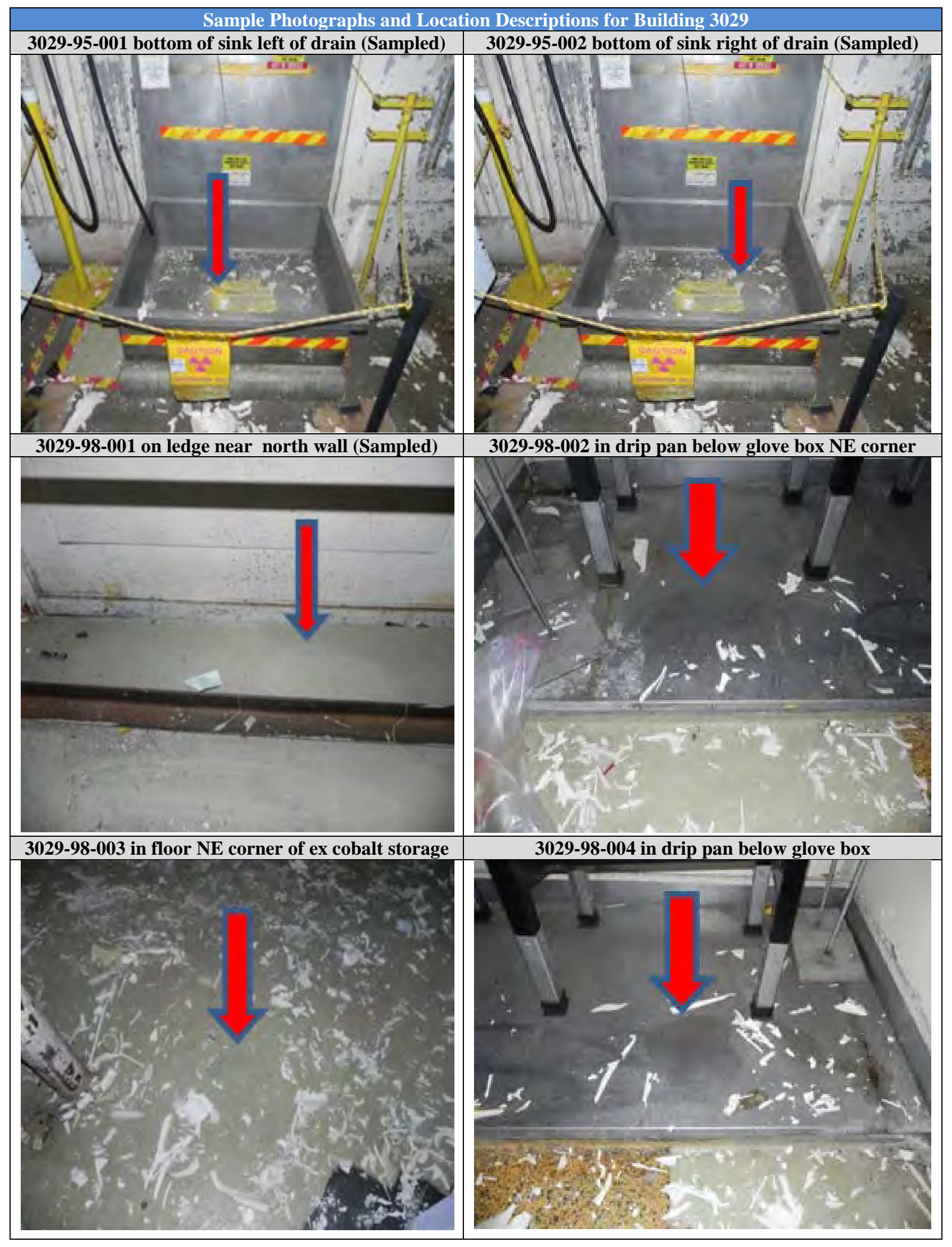




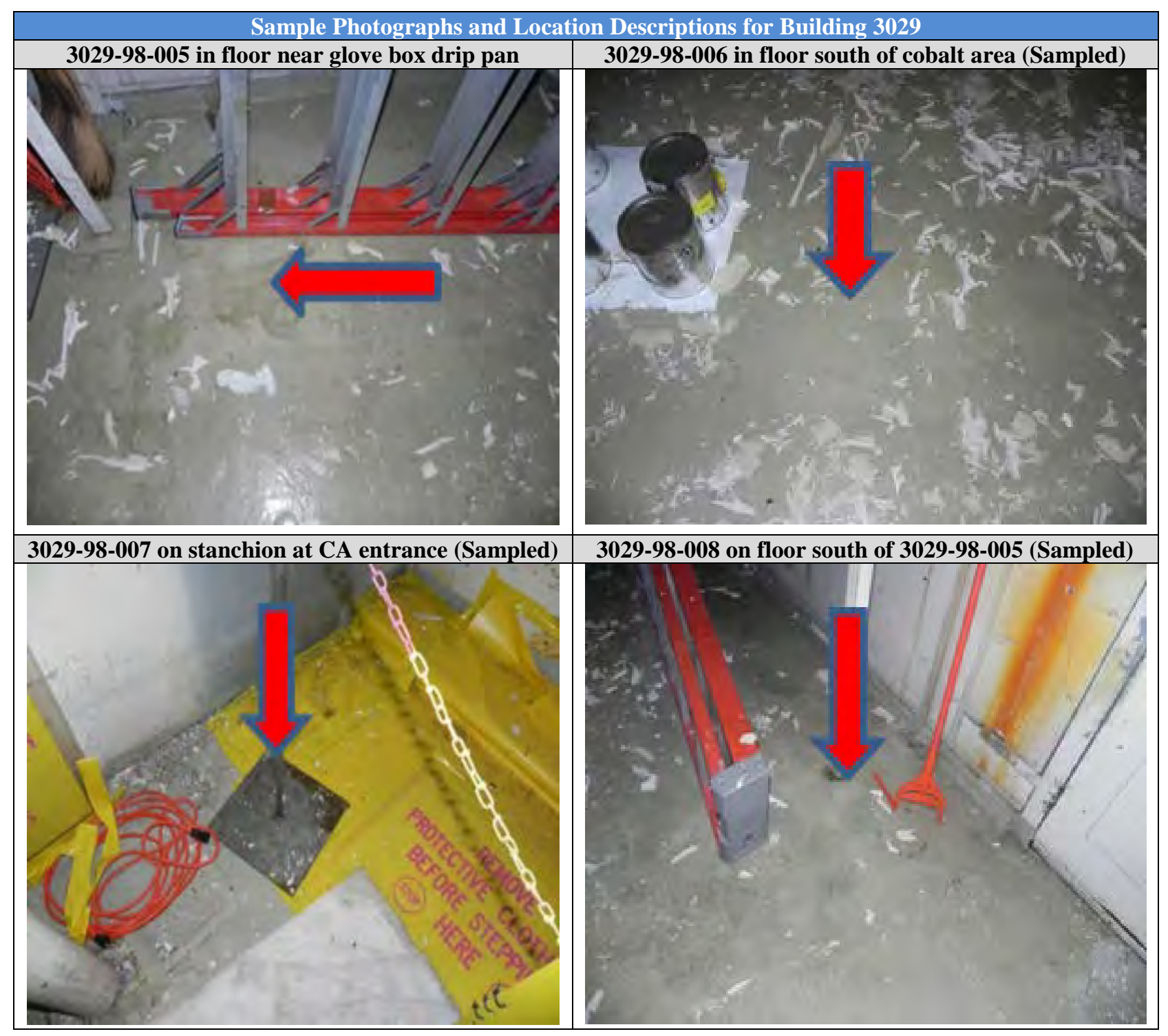




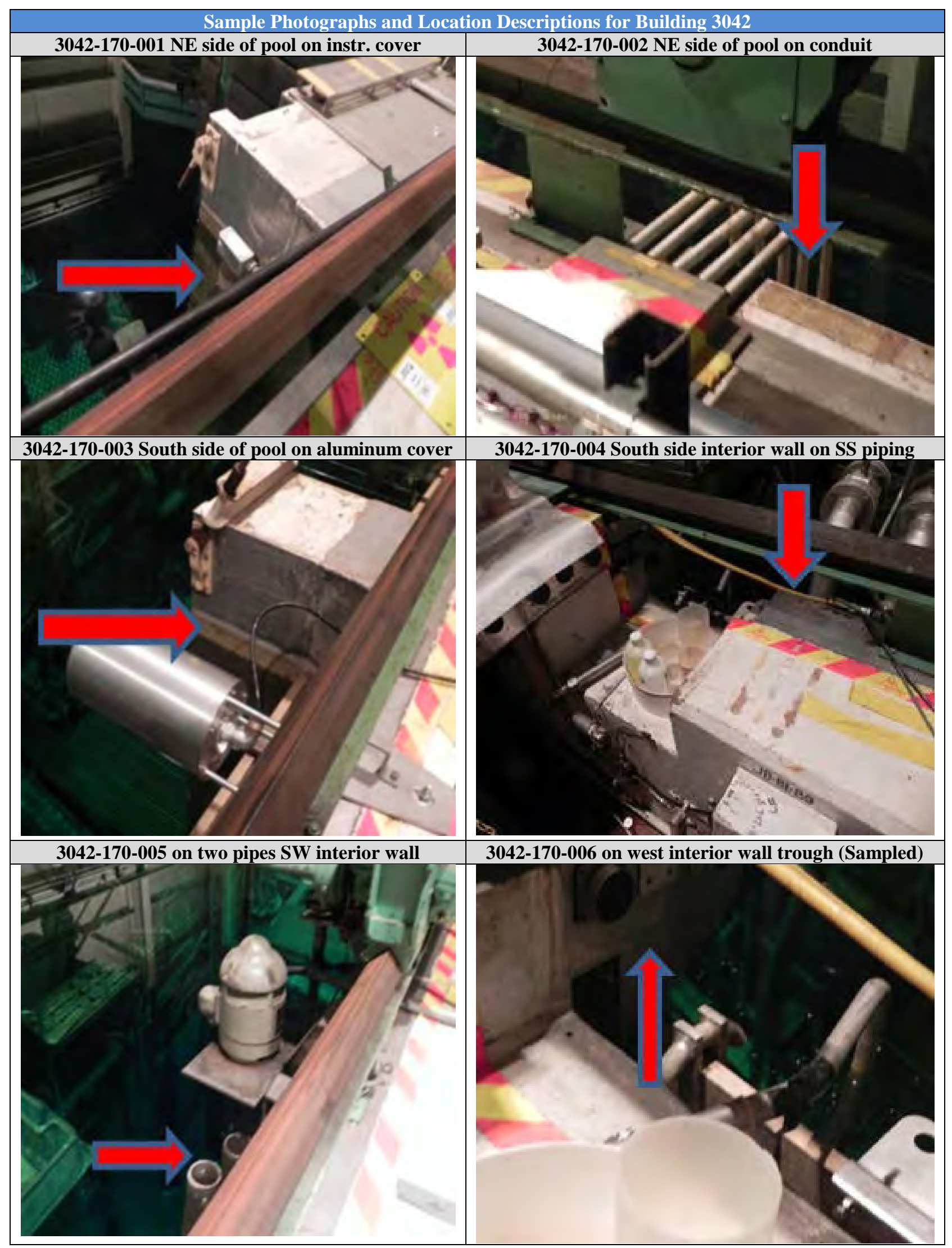




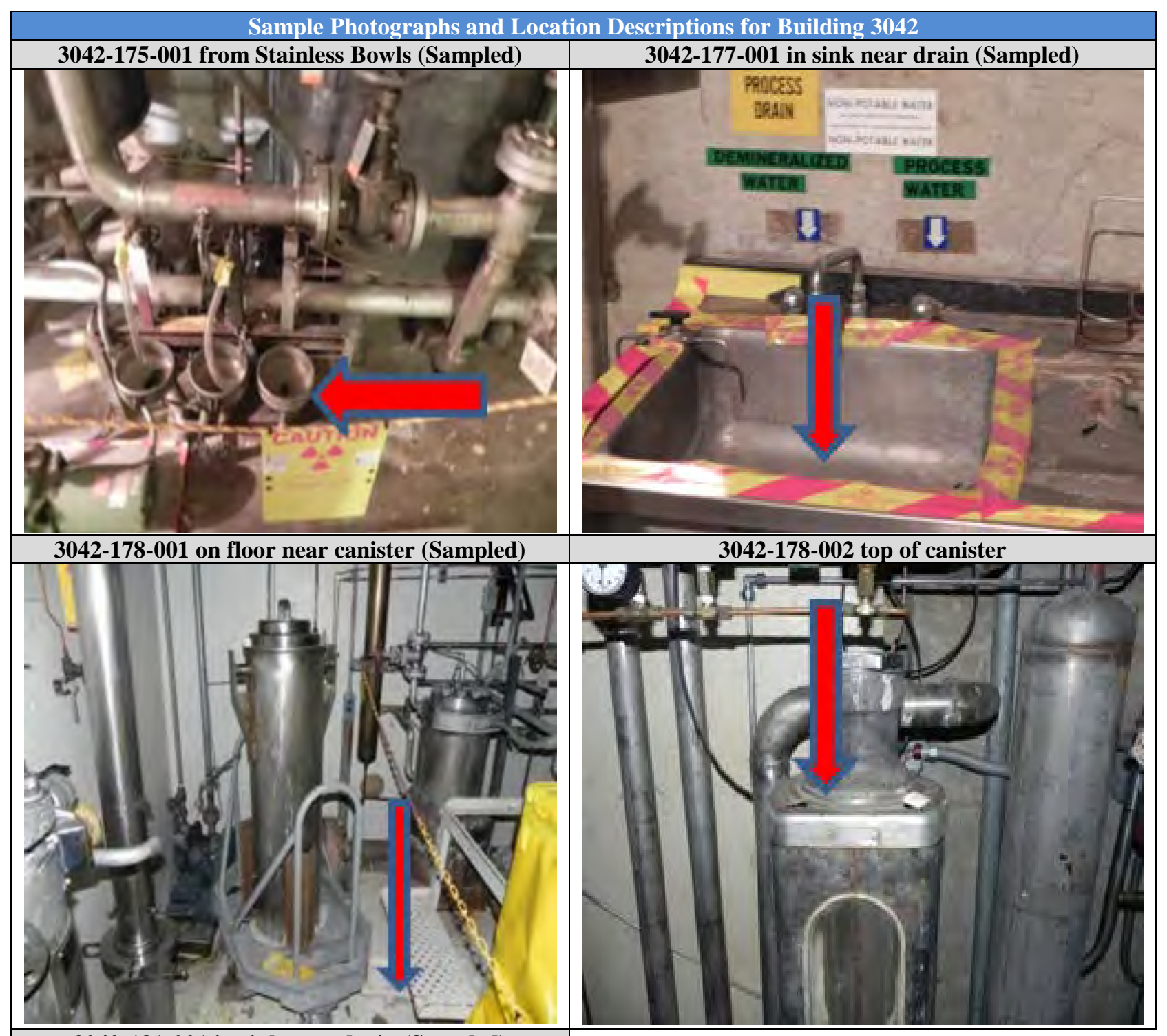

3042-181-001 in sink near drain (Sampled)

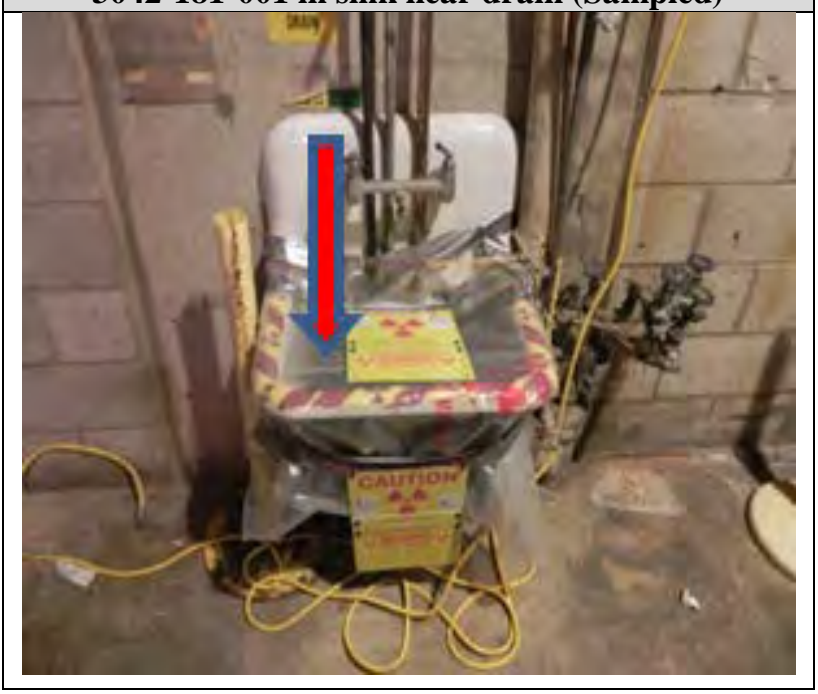


Sample Photographs and Location Descriptions for Building 3517

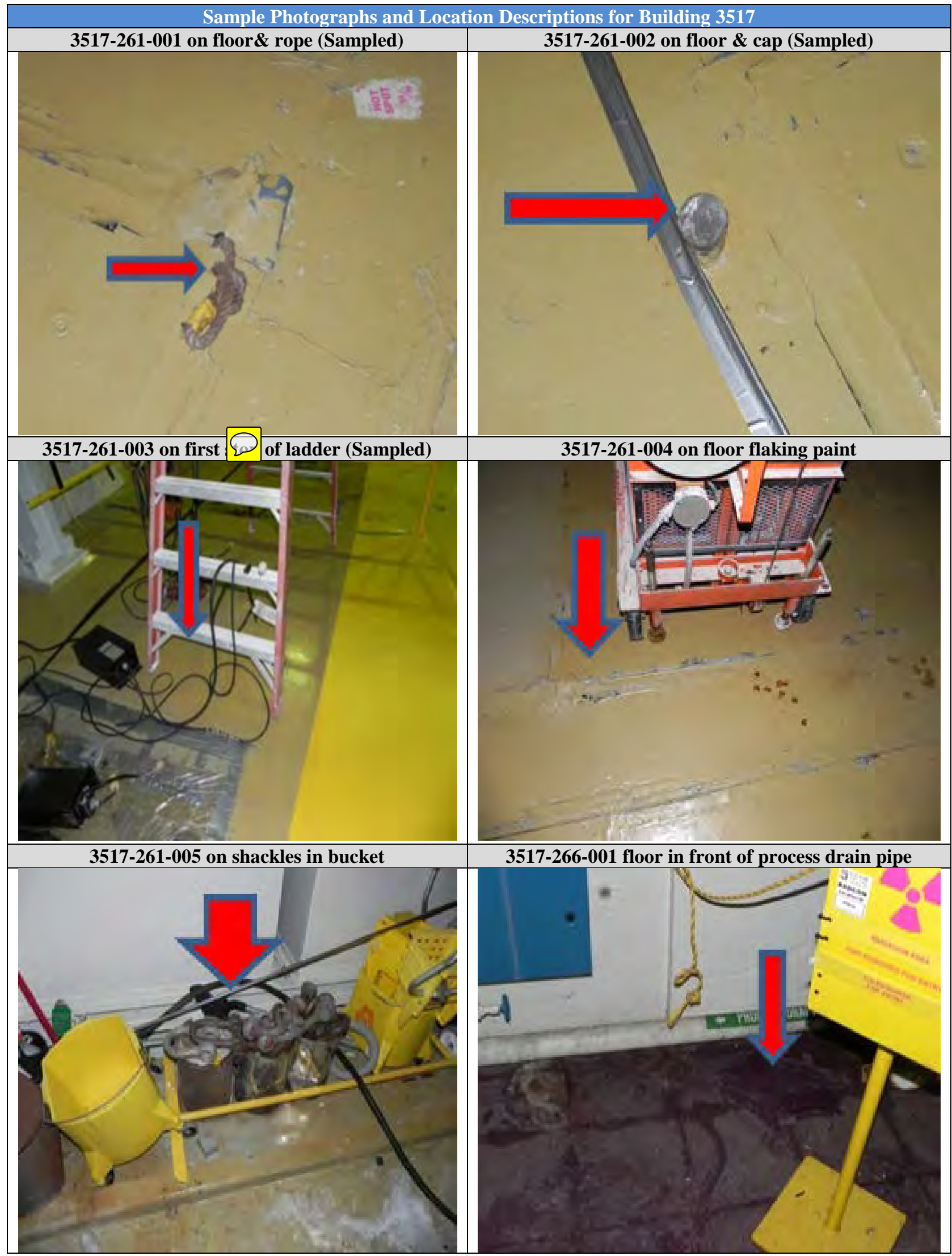


Sample Photographs and Location Descriptions for Building 3517

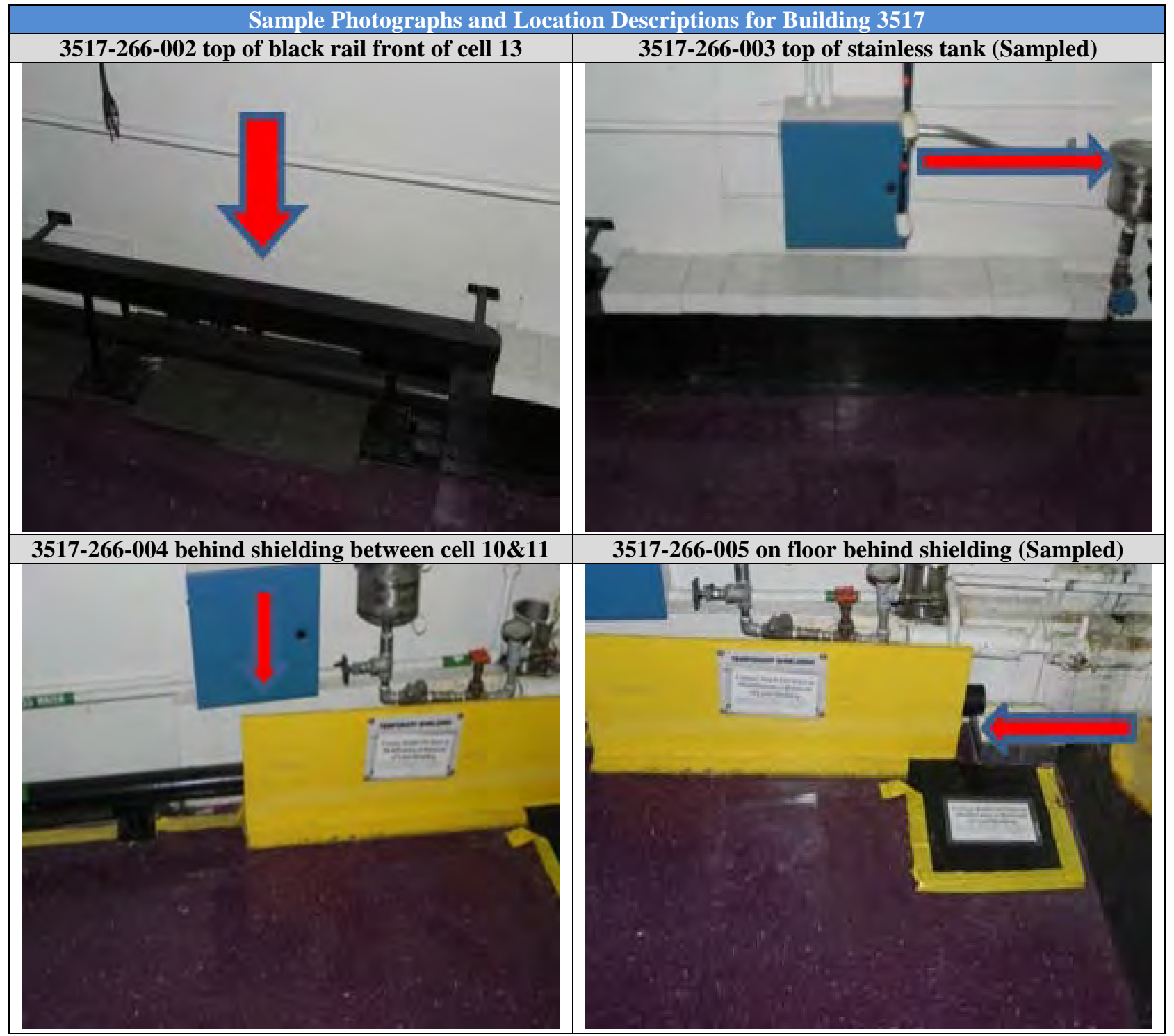




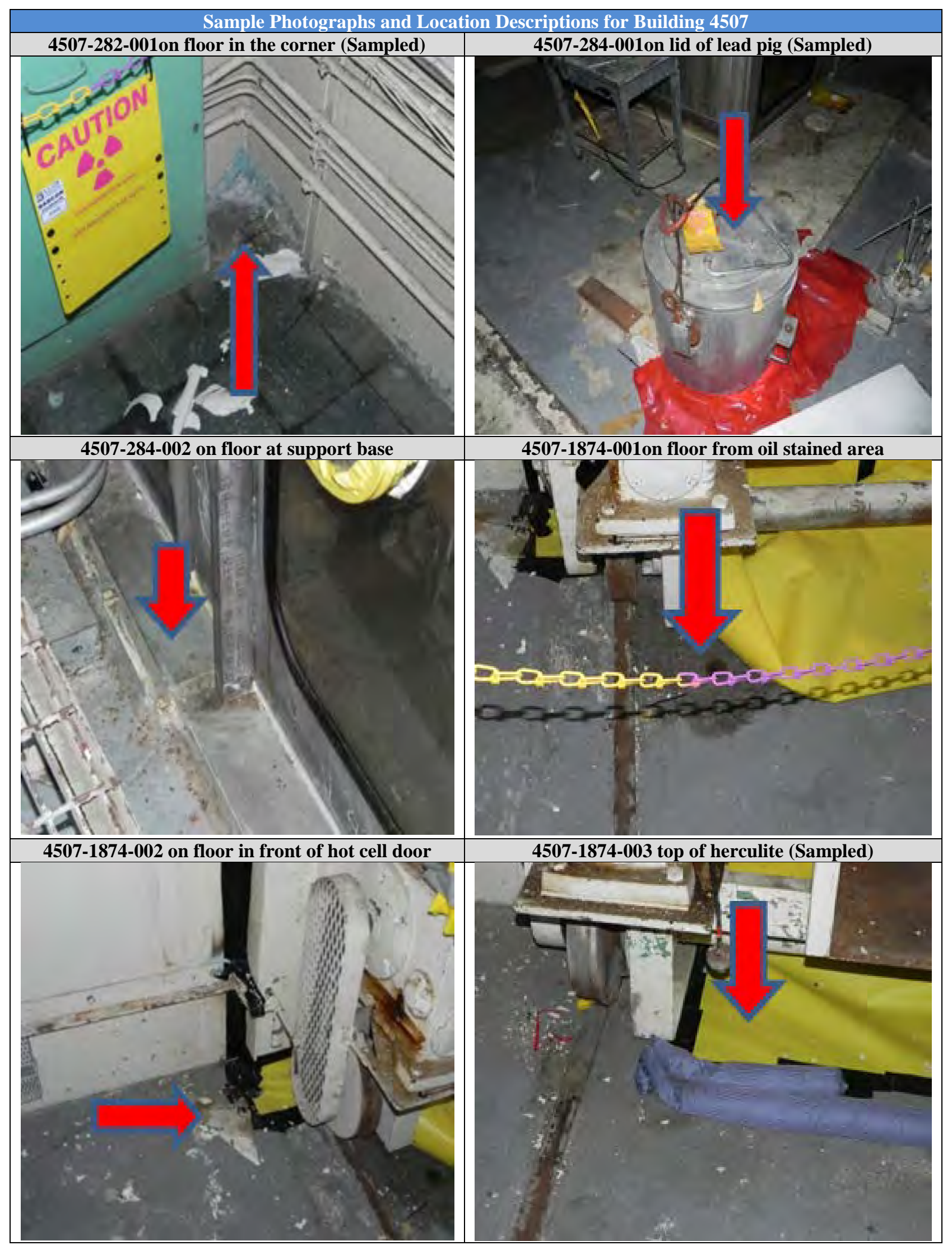




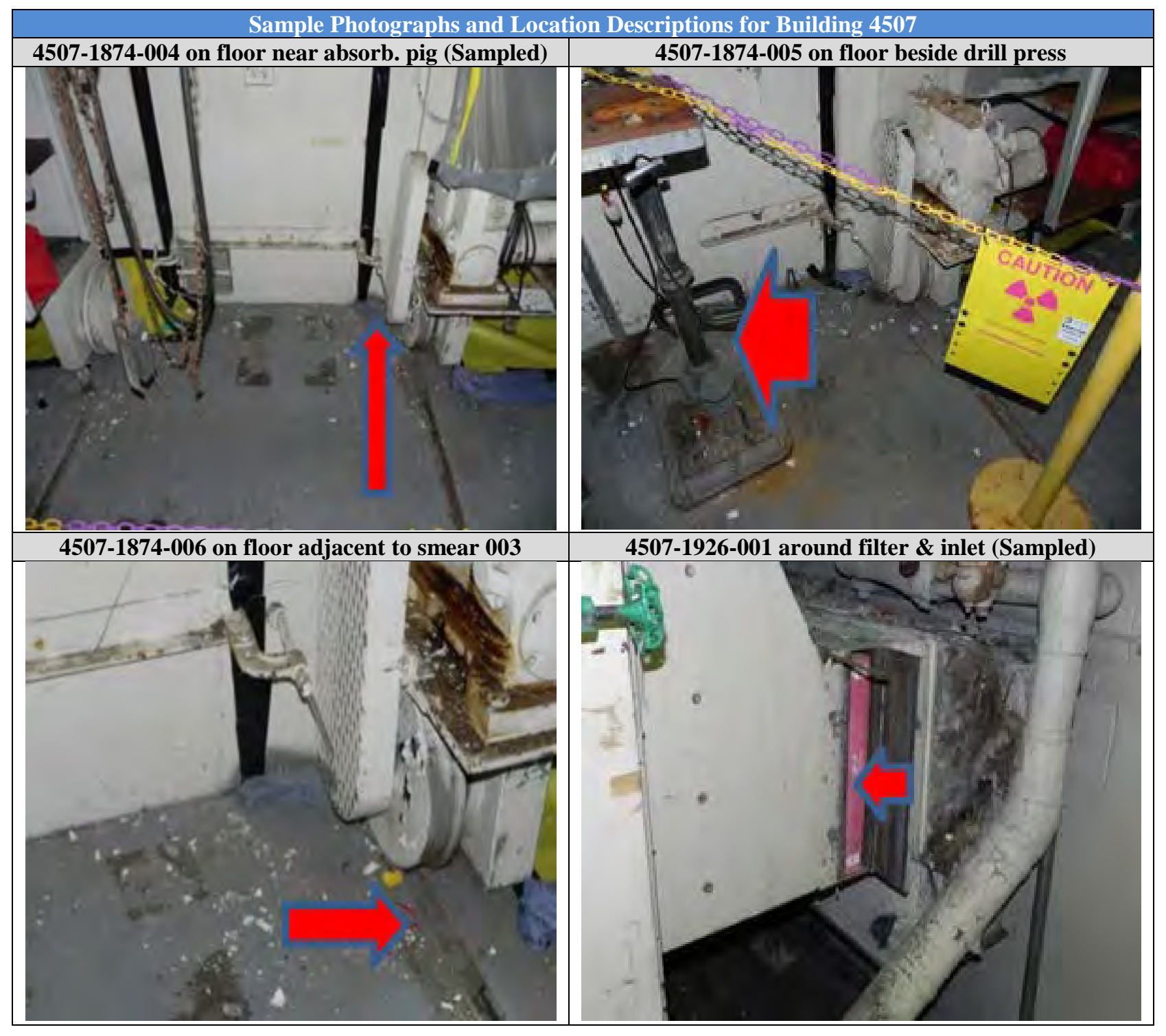


APPENDIX C

STATISTICAL SUMMARY OF LABORATORY ANALYTICAL RESULTS 


\begin{tabular}{|c|c|c|c|c|c|c|c|c|}
\hline \begin{tabular}{|l|} 
Bldg \\
ID
\end{tabular} & Radionuclide Name & $\begin{array}{l}\text { Freq. } \\
\text { of Det. }\end{array}$ & Units & $\begin{array}{l}\text { Det. } \\
\text { Min } \\
\end{array}$ & $\begin{array}{l}\text { Det. } \\
\text { Max }\end{array}$ & $\begin{array}{l}\text { Det. } \\
\text { Ave }\end{array}$ & $\begin{array}{c}\text { Det. } \\
\text { Median }\end{array}$ & $\begin{array}{c}\text { Det. } \\
\text { StdDev }\end{array}$ \\
\hline 3001 & Americium-241 & $3 / 4$ & $\mathrm{pCi} /$ sample & 2.76 & 7.35 & 5.12 & 5.25 & 2.30 \\
\hline 3001 & Americium-243 & $0 / 4$ & $\mathrm{pCi} /$ sample & & & & & \\
\hline 3001 & Carbon-14 & $0 / 4$ & pCi/sample & & & & & \\
\hline 3001 & Cesium-137 & $3 / 4$ & $\mathrm{pCi} /$ sample & 845 & 3010 & 2100 & 2440 & 1120 \\
\hline 3001 & Cobalt-60 & $0 / 4$ & $\mathrm{pCi} /$ sample & & & & & \\
\hline 3001 & Curium-242 & $0 / 4$ & pCi/sample & & & & & \\
\hline 3001 & Curium-243/244 & $0 / 4$ & pCi/sample & & & & & \\
\hline 3001 & Europium-152 & $0 / 4$ & $\mathrm{pCi} /$ sample & & & & & \\
\hline 3001 & Europium-154 & $0 / 4$ & $\mathrm{pCi} /$ sample & & & & & \\
\hline 3001 & Iodine-129 & $0 / 4$ & pCi/sample & & & & & \\
\hline 3001 & Neptunium-237 & $0 / 4$ & $\mathrm{pCi} /$ sample & & & & & \\
\hline 3001 & Nickel-63 & $0 / 4$ & pCi/sample & & & & & \\
\hline 3001 & Plutonium-238 & $0 / 4$ & pCi/sample & & & & & \\
\hline 3001 & Plutonium-239/240 & $3 / 4$ & $\mathrm{pCi} /$ sample & 12.9 & 60.3 & 40.7 & 49.0 & 24.8 \\
\hline 3001 & Protactinium-231 & $0 / 4$ & pCi/sample & & & & & \\
\hline 3001 & Radioactive Strontium (Total) & $3 / 4$ & $\mathrm{pCi} /$ sample & 627 & 2630 & 1440 & 1060 & 1050 \\
\hline 3001 & Radium-226 & $0 / 4$ & pCi/sample & & & & & \\
\hline 3001 & Technetium-99 & $4 / 4$ & pCi/sample & 5.72 & 8.61 & 7.87 & 8.57 & 1.43 \\
\hline 3001 & Thorium-230 & $3 / 4$ & pCi/sample & 4.75 & 5.69 & 5.28 & 5.41 & 0.483 \\
\hline 3001 & Thorium-232 & $0 / 4$ & pCi/sample & & & & & \\
\hline 3001 & Tritium & $0 / 4$ & $\mathrm{pCi} /$ sample & & & & & \\
\hline 3001 & Uranium-233/234 & $1 / 4$ & $\mathrm{pCi} /$ sample & 11.7 & 11.7 & 11.7 & 11.7 & \\
\hline 3001 & Uranium-235/236 & $0 / 4$ & pCi/sample & & & & & \\
\hline 3001 & Uranium-238 & $0 / 4$ & pCi/sample & & & & & \\
\hline 3010 & Americium-241 & $0 / 4$ & pCi/sample & & & & & \\
\hline 3010 & Americium-243 & $0 / 4$ & $\mathrm{pCi} /$ sample & & & & & \\
\hline 3010 & Carbon-14 & $4 / 4$ & $\mathrm{pCi} /$ sample & 236 & 3130 & 1070 & 458 & 1380 \\
\hline 3010 & Cesium-137 & $2 / 4$ & pCi/sample & 64.1 & 177 & 121 & 121 & 79.8 \\
\hline 3010 & Cobalt-60 & $4 / 4$ & pCi/sample & 293 & 2020 & 946 & 735 & 753 \\
\hline 3010 & Curium-242 & $1 / 4$ & pCi/sample & 1.82 & 1.82 & 1.82 & 1.82 & \\
\hline 3010 & Curium-243/244 & $0 / 4$ & $\mathrm{pCi} /$ sample & & & & & \\
\hline 3010 & Europium-152 & $0 / 4$ & pCi/sample & & & & & \\
\hline 3010 & Europium-154 & $0 / 4$ & $\mathrm{pCi} /$ sample & & & & & \\
\hline 3010 & Iodine-129 & $2 / 4$ & pCi/sample & 4.31 & 8.30 & 6.31 & 6.31 & 2.82 \\
\hline 3010 & Neptunium-237 & $0 / 4$ & pCi/sample & & & & & \\
\hline 3010 & Nickel-63 & $4 / 4$ & pCi/sample & 364 & 1760 & 1240 & 1410 & 622 \\
\hline 3010 & Plutonium-238 & $0 / 4$ & pCi/sample & & & & & \\
\hline 3010 & Plutonium-239/240 & $0 / 4$ & pCi/sample & & & & & \\
\hline 3010 & Protactinium-231 & $0 / 4$ & $\mathrm{pCi} /$ sample & & & & & \\
\hline 3010 & Radioactive Strontium (Total) & $1 / 4$ & pCi/sample & 28.6 & 28.6 & 28.6 & 28.6 & \\
\hline 3010 & Radium-226 & $1 / 4$ & pCi/sample & 92.2 & 92.2 & 92.2 & 92.2 & \\
\hline 3010 & Technetium-99 & $4 / 4$ & $\mathrm{pCi} /$ sample & 342 & 2960 & 1410 & 1170 & 1170 \\
\hline 3010 & Thorium-230 & $2 / 4$ & $\mathrm{pCi} /$ sample & 2.62 & 24.5 & 13.6 & 13.6 & 15.5 \\
\hline 3010 & Thorium-232 & $0 / 4$ & pCi/sample & & & & & \\
\hline 3010 & Tritium & $0 / 4$ & pCi/sample & & & & & \\
\hline 3010 & Uranium-233/234 & $2 / 4$ & pCi/sample & 4.53 & 7.91 & 6.22 & 6.22 & 2.39 \\
\hline 3010 & Uranium-235/236 & $0 / 4$ & pCi/sample & & & & & \\
\hline 3010 & Uranium-238 & $1 / 4$ & $\mathrm{pCi} /$ sample & 9.85 & 9.85 & 9.85 & 9.85 & \\
\hline
\end{tabular}




\begin{tabular}{|c|c|c|c|c|c|c|c|c|}
\hline \begin{tabular}{|l|} 
Bldg \\
ID
\end{tabular} & Radionuclide Name & $\begin{array}{l}\text { Freq. } \\
\text { of Det. }\end{array}$ & Units & $\begin{array}{l}\text { Det. } \\
\text { Min } \\
\end{array}$ & $\begin{array}{l}\text { Det. } \\
\text { Max }\end{array}$ & $\begin{array}{l}\text { Det. } \\
\text { Ave }\end{array}$ & $\begin{array}{c}\text { Det. } \\
\text { Median }\end{array}$ & $\begin{array}{c}\text { Det. } \\
\text { StdDev }\end{array}$ \\
\hline 3028 & Americium-241 & $6 / 6$ & pCi/sample & 12.6 & 383 & 96.6 & 47.2 & 143 \\
\hline 3028 & Americium-243 & $1 / 6$ & pCi/sample & 2.92 & 2.92 & 2.92 & 2.92 & \\
\hline 3028 & Carbon-14 & $0 / 6$ & pCi/sample & & & & & \\
\hline 3028 & Cesium-137 & $6 / 6$ & $\mathrm{pCi} /$ sample & 6.81 & 35.6 & 19.0 & 16.6 & 12.1 \\
\hline 3028 & Cobalt-60 & $0 / 6$ & $\mathrm{pCi} /$ sample & & & & & \\
\hline 3028 & Curium-242 & $1 / 6$ & pCi/sample & 8.24 & 8.24 & 8.24 & 8.24 & \\
\hline 3028 & Curium-243/244 & $6 / 6$ & pCi/sample & 182 & 15900 & 3370 & 617 & 6200 \\
\hline 3028 & Europium-152 & $0 / 6$ & $\mathrm{pCi} /$ sample & & & & & \\
\hline 3028 & Europium-154 & $0 / 6$ & pCi/sample & & & & & \\
\hline 3028 & Iodine-129 & $0 / 6$ & pCi/sample & & & & & \\
\hline 3028 & Neptunium-237 & $0 / 6$ & $\mathrm{pCi} /$ sample & & & & & \\
\hline 3028 & Nickel-63 & $1 / 6$ & pCi/sample & 172 & 172 & 172 & 172 & \\
\hline 3028 & Plutonium-238 & $5 / 6$ & pCi/sample & 3.76 & 1210 & 300 & 119 & 511 \\
\hline 3028 & Plutonium-239/240 & $4 / 6$ & pCi/sample & 9.79 & 66.7 & 27.0 & 15.8 & 26.6 \\
\hline 3028 & Protactinium-231 & $0 / 6$ & pCi/sample & & & & & \\
\hline 3028 & Radioactive Strontium (Total) & $4 / 6$ & $\mathrm{pCi} /$ sample & 25.1 & 72.2 & 41.6 & 34.6 & 20.9 \\
\hline 3028 & Radium-226 & $0 / 6$ & pCi/sample & & & & & \\
\hline 3028 & Technetium-99 & $1 / 6$ & pCi/sample & 8.65 & 8.65 & 8.65 & 8.65 & \\
\hline 3028 & Thorium-230 & $6 / 6$ & pCi/sample & 2.84 & 5.02 & 4.00 & 4.10 & 0.872 \\
\hline 3028 & Thorium-232 & $0 / 6$ & pCi/sample & & & & & \\
\hline 3028 & Tritium & $0 / 6$ & $\mathrm{pCi} /$ sample & & & & & \\
\hline 3028 & Uranium-233/234 & $2 / 6$ & pCi/sample & 2.50 & 3.33 & 2.92 & 2.92 & 0.587 \\
\hline 3028 & Uranium-235/236 & $0 / 6$ & $\mathrm{pCi} /$ sample & & & & & \\
\hline 3028 & Uranium-238 & $1 / 6$ & pCi/sample & 1.71 & 1.71 & 1.71 & 1.71 & \\
\hline 3029 & Americium-241 & $2 / 6$ & $\mathrm{pCi} /$ sample & 2.83 & 4.52 & 3.68 & 3.68 & 1.20 \\
\hline 3029 & Americium-243 & $0 / 6$ & pCi/sample & & & & & \\
\hline 3029 & Carbon-14 & $0 / 6$ & $\mathrm{pCi} /$ sample & & & & & \\
\hline 3029 & Cesium-137 & $6 / 6$ & pCi/sample & 121 & 47100 & 9050 & 744 & 18800 \\
\hline 3029 & Cobalt-60 & $2 / 6$ & pCi/sample & 19.4 & 27.3 & 23.4 & 23.4 & 5.59 \\
\hline 3029 & Curium-242 & $0 / 6$ & pCi/sample & & & & & \\
\hline 3029 & Curium-243/244 & $3 / 6$ & pCi/sample & 10.3 & 181 & 74.9 & 33.3 & 92.6 \\
\hline 3029 & Europium-152 & $0 / 6$ & pCi/sample & & & & & \\
\hline 3029 & Europium-154 & $0 / 6$ & $\mathrm{pCi} /$ sample & & & & & \\
\hline 3029 & Iodine-129 & $0 / 6$ & pCi/sample & & & & & \\
\hline 3029 & Neptunium-237 & $0 / 6$ & pCi/sample & & & & & \\
\hline 3029 & Nickel-63 & $2 / 6$ & pCi/sample & 895 & 23800 & 12300 & 12300 & 16200 \\
\hline 3029 & Plutonium-238 & $1 / 6$ & pCi/sample & 19.3 & 19.3 & 19.3 & 19.3 & \\
\hline 3029 & Plutonium-239/240 & $0 / 6$ & pCi/sample & & & & & \\
\hline 3029 & Protactinium-231 & $0 / 6$ & $\mathrm{pCi} /$ sample & & & & & \\
\hline 3029 & Radioactive Strontium (Total) & $5 / 6$ & pCi/sample & 47.4 & 1570 & 373 & 69.3 & 670 \\
\hline 3029 & Radium-226 & $0 / 6$ & pCi/sample & & & & & \\
\hline 3029 & Technetium-99 & $0 / 6$ & $\mathrm{pCi} /$ sample & & & & & \\
\hline 3029 & Thorium-230 & $4 / 6$ & $\mathrm{pCi} /$ sample & 4.71 & 8.39 & 6.07 & 5.60 & 1.62 \\
\hline 3029 & Thorium-232 & $0 / 6$ & pCi/sample & & & & & \\
\hline 3029 & Tritium & $0 / 6$ & $\mathrm{pCi} /$ sample & & & & & \\
\hline 3029 & Uranium-233/234 & $1 / 6$ & pCi/sample & 2.27 & 2.27 & 2.27 & 2.27 & \\
\hline 3029 & Uranium-235/236 & $0 / 6$ & pCi/sample & & & & & \\
\hline 3029 & Uranium-238 & $0 / 6$ & $\mathrm{pCi} /$ sample & & & & & \\
\hline
\end{tabular}




\begin{tabular}{|c|c|c|c|c|c|c|c|c|}
\hline $\begin{array}{l}\text { Bldg } \\
\text { ID }\end{array}$ & Radionuclide Name & $\begin{array}{l}\text { Freq. } \\
\text { of Det. }\end{array}$ & Units & $\begin{array}{l}\text { Det. } \\
\text { Min }\end{array}$ & $\begin{array}{l}\text { Det. } \\
\text { Max }\end{array}$ & $\begin{array}{l}\text { Det. } \\
\text { Ave }\end{array}$ & $\begin{array}{c}\text { Det. } \\
\text { Median }\end{array}$ & $\begin{array}{c}\text { Det. } \\
\text { StdDev }\end{array}$ \\
\hline 3038 & Americium-241 & $5 / 5$ & pCi/sample & 2.49 & 754 & 159 & 10.6 & 333 \\
\hline 3038 & Americium-243 & $0 / 5$ & $\mathrm{pCi} /$ sample & & & & & \\
\hline 3038 & Carbon-14 & $0 / 5$ & pCi/sample & & & & & \\
\hline 3038 & Cesium-137 & $3 / 5$ & pCi/sample & 7.16 & 351 & 130 & 32.2 & 192 \\
\hline 3038 & Cobalt-60 & $0 / 5$ & pCi/sample & & & & & \\
\hline 3038 & Curium-242 & $0 / 5$ & pCi/sample & & & & & \\
\hline 3038 & Curium-243/244 & $0 / 5$ & pCi/sample & & & & & \\
\hline 3038 & Europium-152 & $0 / 5$ & $\mathrm{pCi} /$ sample & & & & & \\
\hline 3038 & Europium-154 & $0 / 5$ & pCi/sample & & & & & \\
\hline 3038 & Iodine-129 & $0 / 5$ & pCi/sample & & & & & \\
\hline 3038 & Neptunium-237 & $0 / 5$ & $\mathrm{pCi} /$ sample & & & & & \\
\hline 3038 & Nickel-63 & $0 / 5$ & pCi/sample & & & & & \\
\hline 3038 & Plutonium-238 & $1 / 5$ & pCi/sample & 22.2 & 22.2 & 22.2 & 22.2 & \\
\hline 3038 & Plutonium-239/240 & $0 / 5$ & pCi/sample & & & & & \\
\hline 3038 & Protactinium-231 & $0 / 5$ & pCi/sample & & & & & \\
\hline 3038 & Radioactive Strontium (Total) & $1 / 5$ & $\mathrm{pCi} /$ sample & 91.9 & 91.9 & 91.9 & 91.9 & \\
\hline 3038 & Radium-226 & $0 / 5$ & $\mathrm{pCi} /$ sample & & & & & \\
\hline 3038 & Technetium-99 & $1 / 5$ & pCi/sample & 8.59 & 8.59 & 8.59 & 8.59 & \\
\hline 3038 & Thorium-230 & $4 / 5$ & pCi/sample & 2.17 & 5.36 & 3.91 & 4.06 & 1.32 \\
\hline 3038 & Thorium-232 & $0 / 5$ & pCi/sample & & & & & \\
\hline 3038 & Tritium & $0 / 5$ & $\mathrm{pCi} /$ sample & & & & & \\
\hline 3038 & Uranium-233/234 & $1 / 5$ & $\mathrm{pCi} /$ sample & 1.76 & 1.76 & 1.76 & 1.76 & \\
\hline 3038 & Uranium-235/236 & $0 / 5$ & pCi/sample & & & & & \\
\hline 3038 & Uranium-238 & $0 / 5$ & pCi/sample & & & & & \\
\hline 3042 & Americium-241 & $4 / 5$ & pCi/sample & 3.82 & 3620 & 926 & 39.8 & 1800 \\
\hline 3042 & Americium-243 & $0 / 5$ & $\mathrm{pCi} /$ sample & & & & & \\
\hline 3042 & Carbon-14 & $1 / 5$ & $\mathrm{pCi} /$ sample & 1280 & 1280 & 1280 & 1280 & \\
\hline 3042 & Cesium-137 & $5 / 5$ & pCi/sample & 150 & 427000 & 91600 & 3190 & 188000 \\
\hline 3042 & Cobalt-60 & $2 / 5$ & pCi/sample & 78.8 & 22000 & 11000 & 11000 & 15500 \\
\hline 3042 & Curium-242 & $0 / 5$ & pCi/sample & & & & & \\
\hline 3042 & Curium-243/244 & $2 / 5$ & pCi/sample & 2.70 & 1050 & 526 & 526 & 741 \\
\hline 3042 & Europium-152 & $2 / 5$ & pCi/sample & 236 & 75500 & 37900 & 37900 & 53200 \\
\hline 3042 & Europium-154 & $1 / 5$ & $\mathrm{pCi} /$ sample & 19400 & 19400 & 19400 & 19400 & \\
\hline 3042 & Iodine-129 & $2 / 5$ & pCi/sample & 19.0 & 453 & 236 & 236 & 307 \\
\hline 3042 & Neptunium-237 & $0 / 5$ & pCi/sample & & & & & \\
\hline 3042 & Nickel-63 & $2 / 5$ & pCi/sample & 442 & 36900 & 18700 & 18700 & 25800 \\
\hline 3042 & Plutonium-238 & $3 / 5$ & pCi/sample & 4.71 & 607 & 240 & 108 & 322 \\
\hline 3042 & Plutonium-239/240 & $2 / 5$ & pCi/sample & 28.5 & 111 & 69.8 & 69.8 & 58.3 \\
\hline 3042 & Protactinium-231 & $0 / 5$ & $\mathrm{pCi} /$ sample & & & & & \\
\hline 3042 & Radioactive Strontium (Total) & $4 / 5$ & pCi/sample & 25.8 & 12200 & 3110 & 105 & 6060 \\
\hline 3042 & Radium-226 & $0 / 5$ & pCi/sample & & & & & \\
\hline 3042 & Technetium-99 & $5 / 5$ & $\mathrm{pCi} /$ sample & 5.81 & 357 & 77.7 & 8.62 & 156 \\
\hline 3042 & Thorium-230 & $3 / 5$ & pCi/sample & 3.87 & 6.49 & 4.85 & 4.18 & 1.43 \\
\hline 3042 & Thorium-232 & $0 / 5$ & pCi/sample & & & & & \\
\hline 3042 & Tritium & $0 / 5$ & $\mathrm{pCi} /$ sample & & & & & \\
\hline 3042 & Uranium-233/234 & $2 / 5$ & pCi/sample & 6.44 & 7.01 & 6.73 & 6.73 & 0.403 \\
\hline 3042 & Uranium-235/236 & $0 / 5$ & pCi/sample & & & & & \\
\hline 3042 & Uranium-238 & $0 / 5$ & $\mathrm{pCi} /$ sample & & & & & \\
\hline
\end{tabular}




\begin{tabular}{|c|c|c|c|c|c|c|c|c|}
\hline \begin{tabular}{|l|} 
Bldg \\
ID
\end{tabular} & Radionuclide Name & $\begin{array}{l}\text { Freq. } \\
\text { of Det. }\end{array}$ & Units & $\begin{array}{l}\text { Det. } \\
\text { Min }\end{array}$ & $\begin{array}{l}\text { Det. } \\
\text { Max }\end{array}$ & $\begin{array}{l}\text { Det. } \\
\text { Ave }\end{array}$ & $\begin{array}{c}\text { Det. } \\
\text { Median }\end{array}$ & $\begin{array}{c}\text { Det. } \\
\text { StdDev }\end{array}$ \\
\hline 3517 & Americium-241 & $1 / 5$ & pCi/sample & 66.7 & 66.7 & 66.7 & 66.7 & \\
\hline 3517 & Americium-243 & $0 / 5$ & pCi/sample & & & & & \\
\hline 3517 & Carbon-14 & $0 / 5$ & pCi/sample & & & & & \\
\hline 3517 & Cesium-137 & $5 / 5$ & $\mathrm{pCi} /$ sample & 67.4 & 12100 & 3730 & 1040 & 5140 \\
\hline 3517 & Cobalt-60 & $0 / 5$ & $\mathrm{pCi} /$ sample & & & & & \\
\hline 3517 & Curium-242 & $1 / 5$ & pCi/sample & 9.26 & 9.26 & 9.26 & 9.26 & \\
\hline 3517 & Curium-243/244 & $5 / 5$ & pCi/sample & 4.78 & 14.6 & 8.66 & 8.24 & 3.66 \\
\hline 3517 & Europium-152 & $0 / 5$ & $\mathrm{pCi} /$ sample & & & & & \\
\hline 3517 & Europium-154 & $0 / 5$ & pCi/sample & & & & & \\
\hline 3517 & Iodine-129 & $0 / 5$ & pCi/sample & & & & & \\
\hline 3517 & Neptunium-237 & $0 / 5$ & $\mathrm{pCi} /$ sample & & & & & \\
\hline 3517 & Nickel-63 & $2 / 5$ & pCi/sample & 297 & 369 & 333 & 333 & 50.9 \\
\hline 3517 & Plutonium-238 & $1 / 5$ & pCi/sample & 1.61 & 1.61 & 1.61 & 1.61 & \\
\hline 3517 & Plutonium-239/240 & $0 / 5$ & $\mathrm{pCi} /$ sample & & & & & \\
\hline 3517 & Protactinium-231 & $0 / 5$ & pCi/sample & & & & & \\
\hline 3517 & Radioactive Strontium (Total) & $5 / 5$ & $\mathrm{pCi} /$ sample & 577 & 11100 & 4620 & 1150 & 5280 \\
\hline 3517 & Radium-226 & $0 / 5$ & pCi/sample & & & & & \\
\hline 3517 & Technetium-99 & $0 / 5$ & pCi/sample & & & & & \\
\hline 3517 & Thorium-230 & $4 / 5$ & pCi/sample & 3.06 & 5.46 & 4.23 & 4.19 & 0.996 \\
\hline 3517 & Thorium-232 & $0 / 5$ & pCi/sample & & & & & \\
\hline 3517 & Tritium & $1 / 5$ & $\mathrm{pCi} /$ sample & 77700 & 77700 & 77700 & 77700 & \\
\hline 3517 & Uranium-233/234 & $2 / 5$ & $\mathrm{pCi} /$ sample & 3.07 & 3.77 & 3.42 & 3.42 & 0.495 \\
\hline 3517 & Uranium-235/236 & $0 / 5$ & pCi/sample & & & & & \\
\hline 3517 & Uranium-238 & $0 / 5$ & pCi/sample & & & & & \\
\hline 4507 & Americium-241 & $4 / 5$ & $\mathrm{pCi} /$ sample & 5.29 & 12.3 & 9.69 & 10.6 & 3.26 \\
\hline 4507 & Americium-243 & $0 / 5$ & pCi/sample & & & & & \\
\hline 4507 & Carbon-14 & $0 / 5$ & $\mathrm{pCi} /$ sample & & & & & \\
\hline 4507 & Cesium-137 & $5 / 5$ & pCi/sample & 11.3 & 1630 & 612 & 127 & 754 \\
\hline 4507 & Cobalt-60 & $0 / 5$ & pCi/sample & & & & & \\
\hline 4507 & Curium-242 & $0 / 5$ & pCi/sample & & & & & \\
\hline 4507 & Curium-243/244 & $3 / 5$ & pCi/sample & 4.52 & 10.5 & 6.54 & 4.60 & 3.43 \\
\hline 4507 & Europium-152 & $0 / 5$ & pCi/sample & & & & & \\
\hline 4507 & Europium-154 & $0 / 5$ & $\mathrm{pCi} /$ sample & & & & & \\
\hline 4507 & Iodine-129 & $0 / 5$ & pCi/sample & & & & & \\
\hline 4507 & Neptunium-237 & $0 / 5$ & pCi/sample & & & & & \\
\hline 4507 & Nickel-63 & $1 / 5$ & $\mathrm{pCi} /$ sample & 181 & 181 & 181 & 181 & \\
\hline 4507 & Plutonium-238 & $2 / 5$ & pCi/sample & 3.22 & 4.57 & 3.9 & 3.9 & 0.955 \\
\hline 4507 & Plutonium-239/240 & $3 / 5$ & pCi/sample & 3.2 & 11.5 & 6.45 & 4.65 & 4.43 \\
\hline 4507 & Protactinium-231 & $0 / 5$ & $\mathrm{pCi} /$ sample & & & & & \\
\hline 4507 & Radioactive Strontium (Total) & $5 / 5$ & pCi/sample & 40.5 & 2080 & 605 & 248 & 836 \\
\hline 4507 & Radium-226 & $0 / 5$ & pCi/sample & & & & & \\
\hline 4507 & Technetium-99 & $2 / 5$ & $\mathrm{pCi} /$ sample & 8.65 & 8.72 & 8.69 & 8.69 & 0.0495 \\
\hline 4507 & Thorium-230 & $3 / 5$ & $\mathrm{pCi} /$ sample & 3.37 & 5.38 & 4.16 & 3.72 & 1.07 \\
\hline 4507 & Thorium-232 & $0 / 5$ & pCi/sample & & & & & \\
\hline 4507 & Tritium & $0 / 5$ & pCi/sample & & & & & \\
\hline 4507 & Uranium-233/234 & $4 / 5$ & pCi/sample & 2.11 & 33.9 & 10.3 & 2.60 & 15.7 \\
\hline 4507 & Uranium-235/236 & $1 / 5$ & pCi/sample & 3.61 & 3.61 & 3.61 & 3.61 & \\
\hline 4507 & Uranium-238 & $0 / 5$ & $\mathrm{pCi} /$ sample & & & & & \\
\hline
\end{tabular}




\begin{tabular}{|c|c|c|c|c|c|c|c|c|}
\hline $\begin{array}{l}\text { Bldg } \\
\text { ID }\end{array}$ & Radionuclide Name & $\begin{array}{l}\text { Freq. } \\
\text { of Det. }\end{array}$ & Units & $\begin{array}{l}\text { Det. } \\
\text { Min }\end{array}$ & $\begin{array}{l}\text { Det. } \\
\text { Max }\end{array}$ & $\begin{array}{l}\text { Det. } \\
\text { Ave }\end{array}$ & $\begin{array}{c}\text { Det. } \\
\text { Median }\end{array}$ & $\begin{array}{c}\text { Det. } \\
\text { StdDev }\end{array}$ \\
\hline
\end{tabular}

All values rounded to three significant digits

Results are only shown for detected radionuclides

Standard Devation only calculated when there is more than one detect 
APPENDIX D

PLOTS OF LABORATORY ANALYTICAL DATA VS. INSTRUMENT

MEASUREMENT DATA 

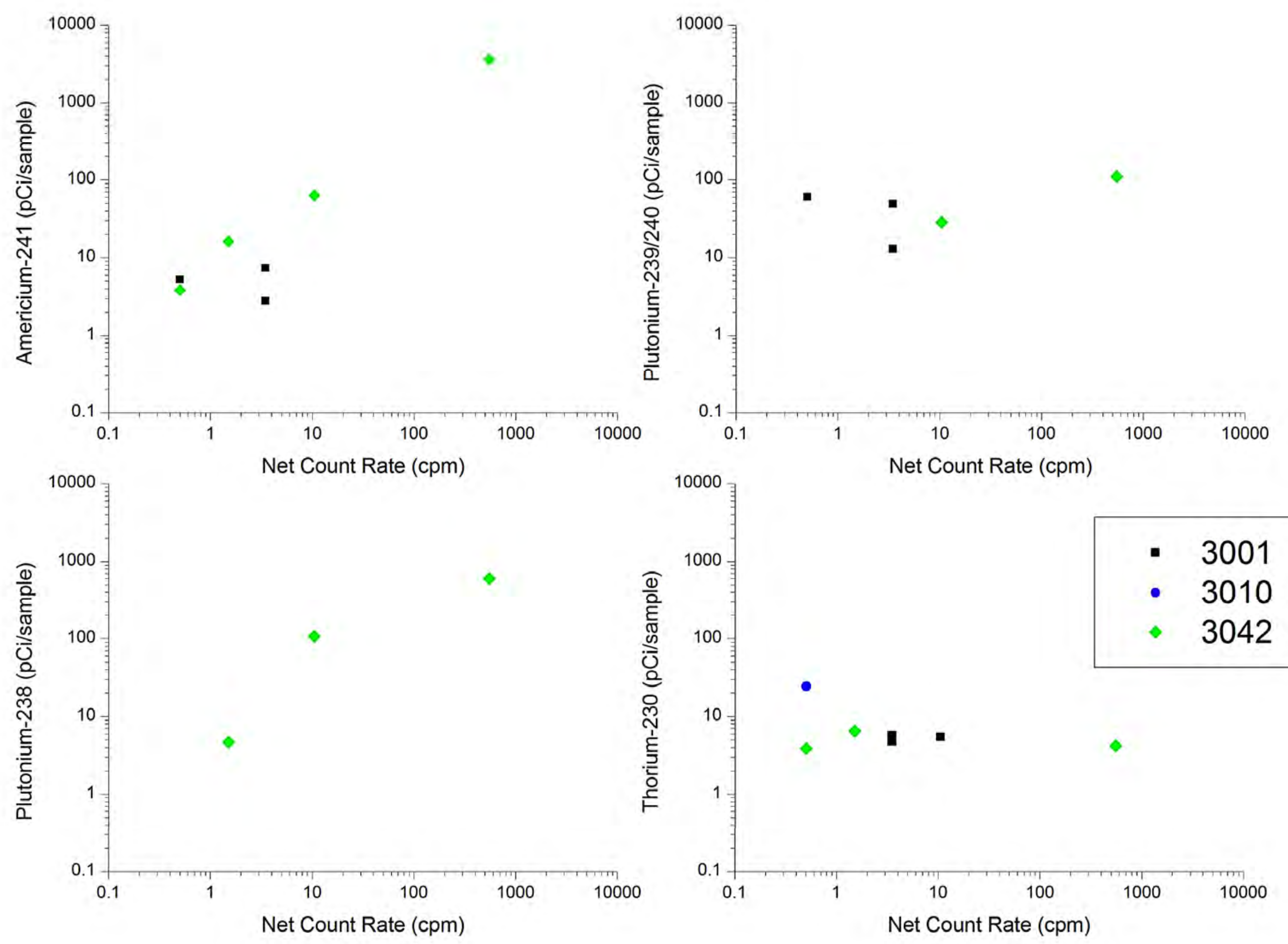


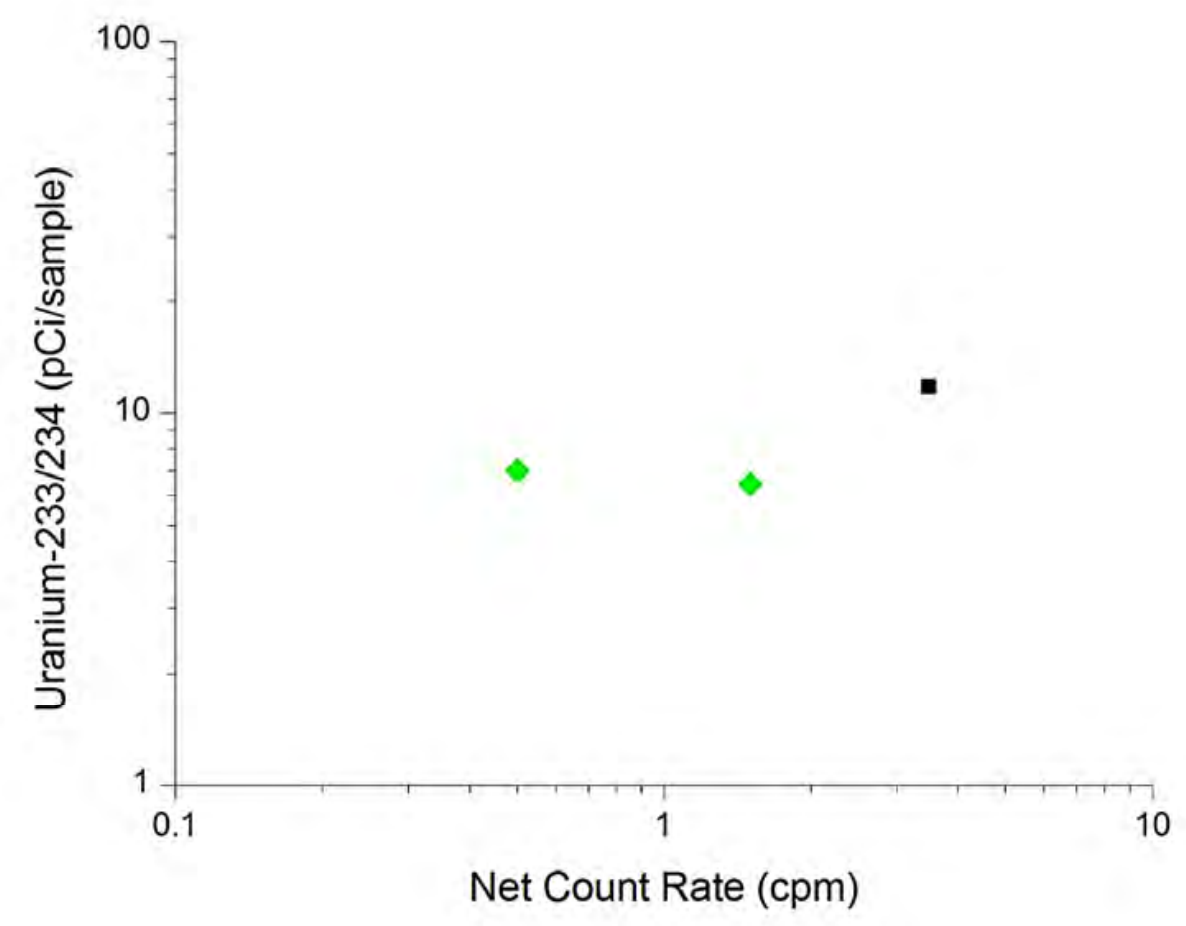
- 3001
- 3010 3042

10 

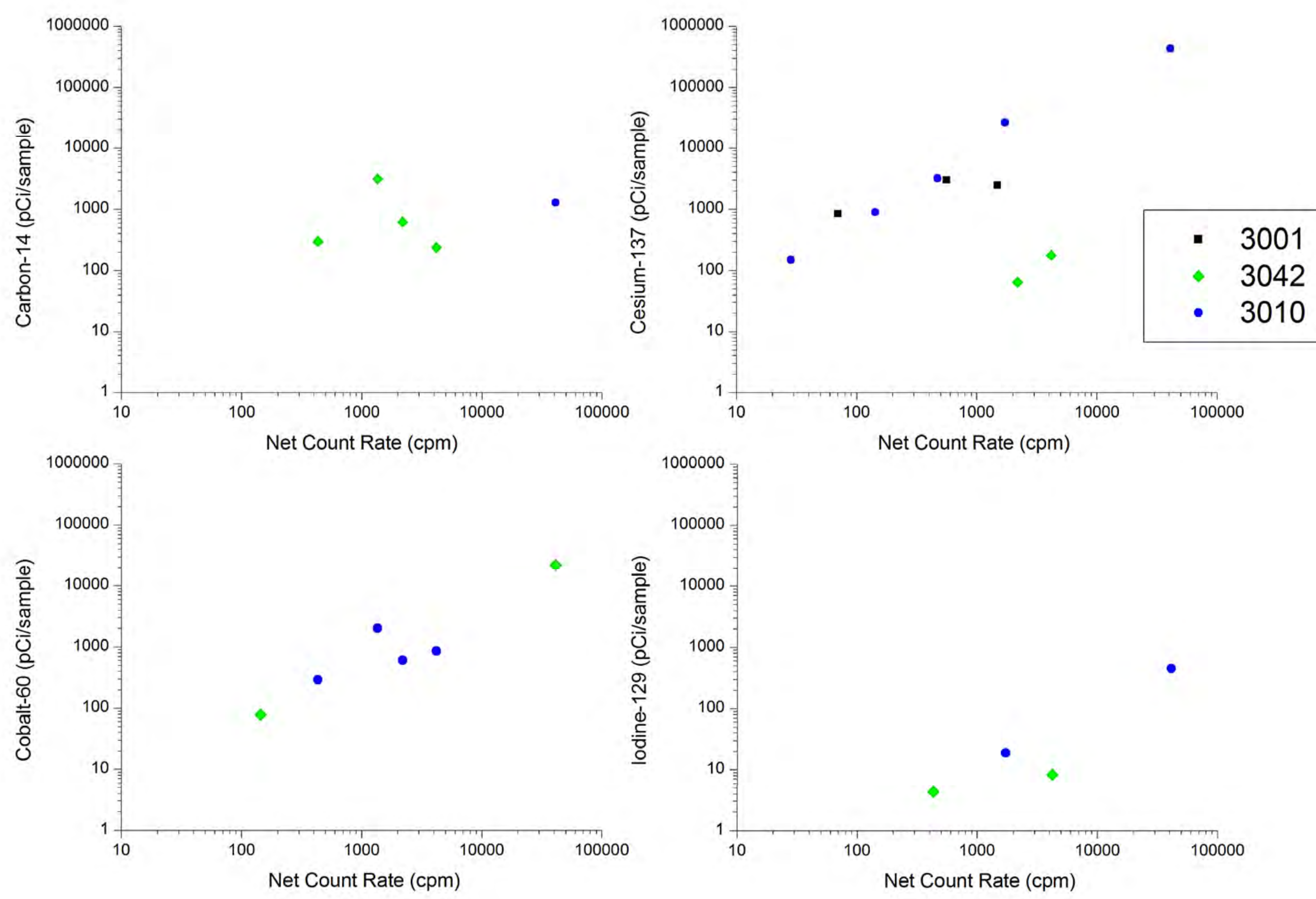

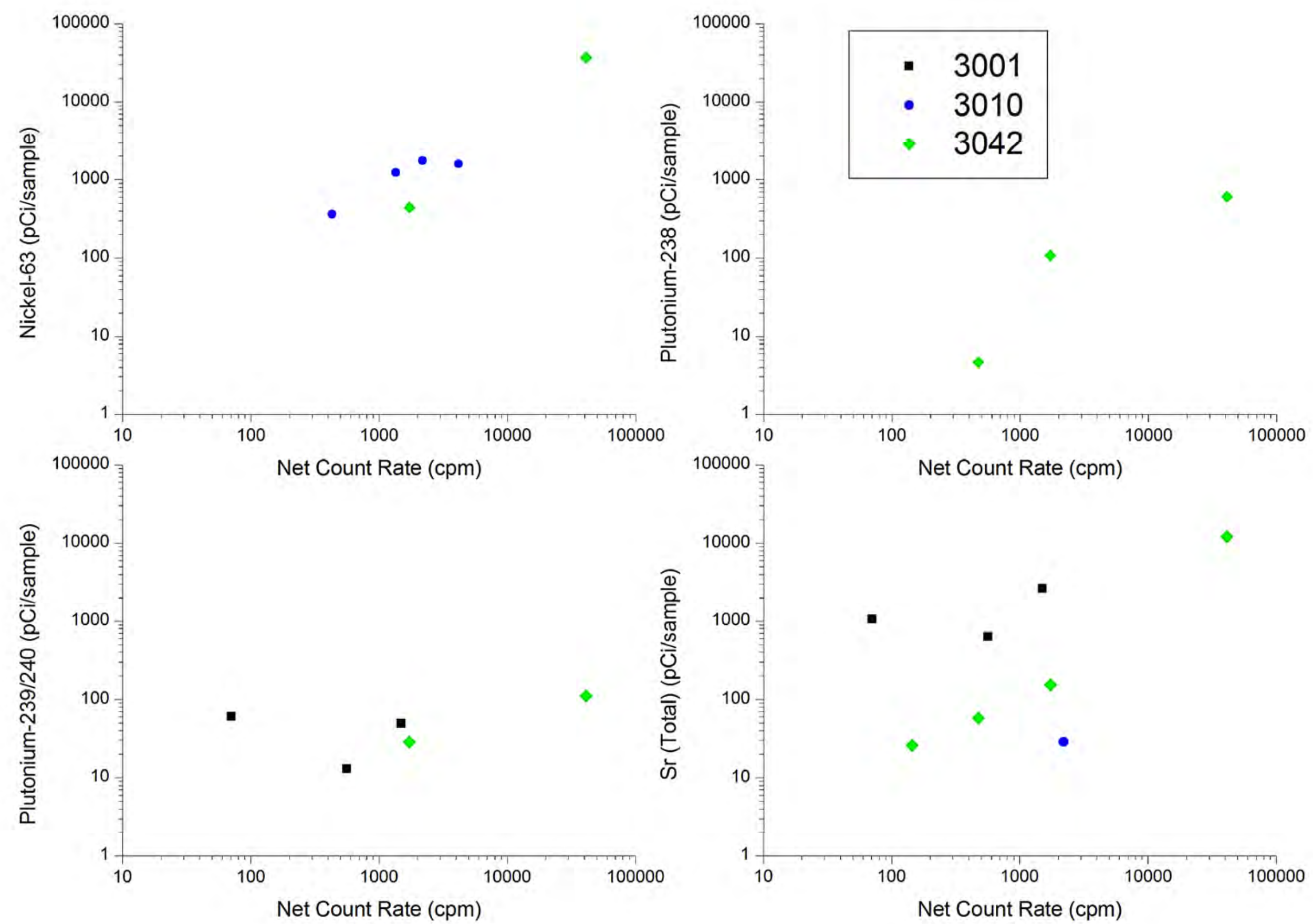

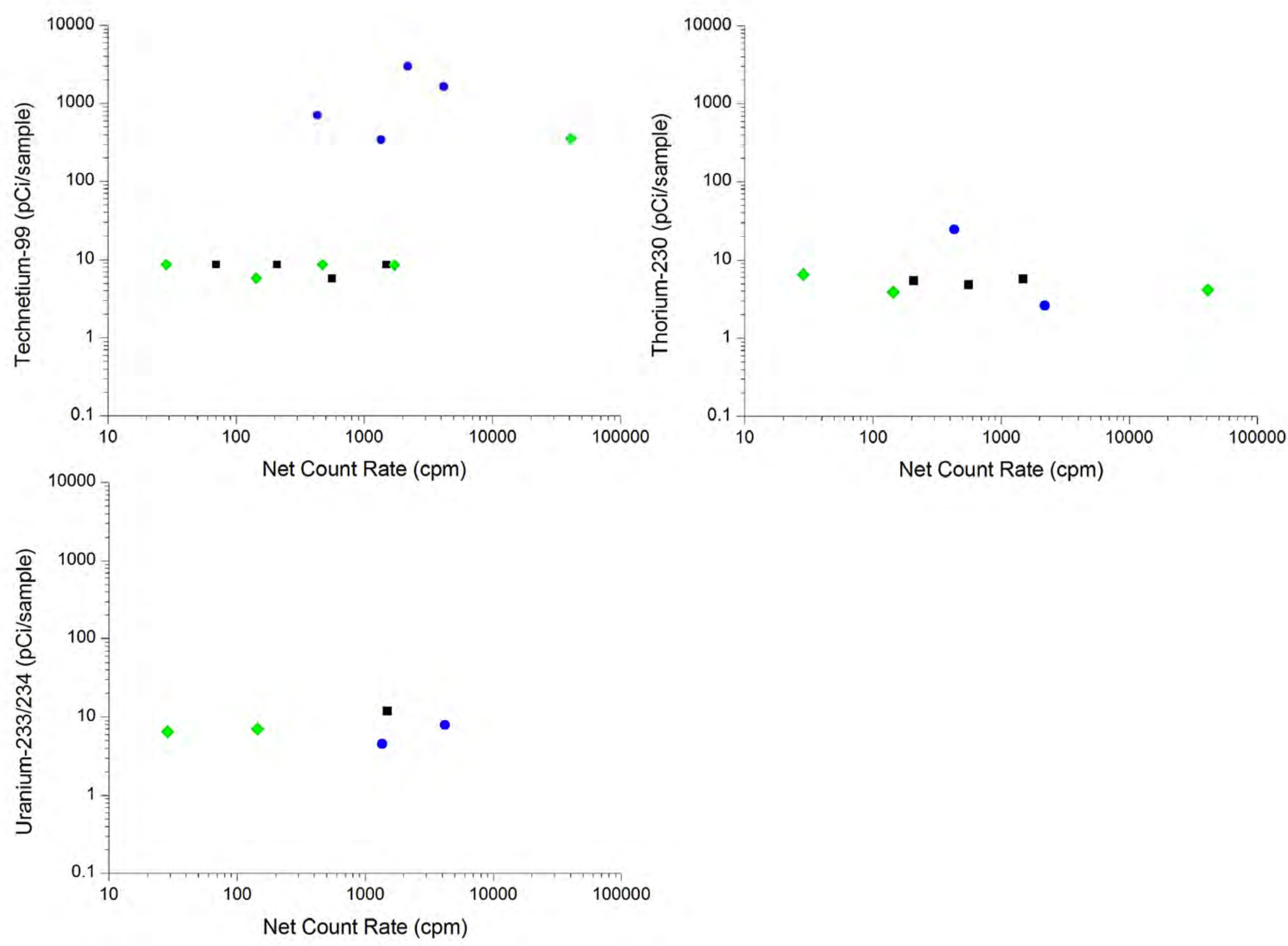

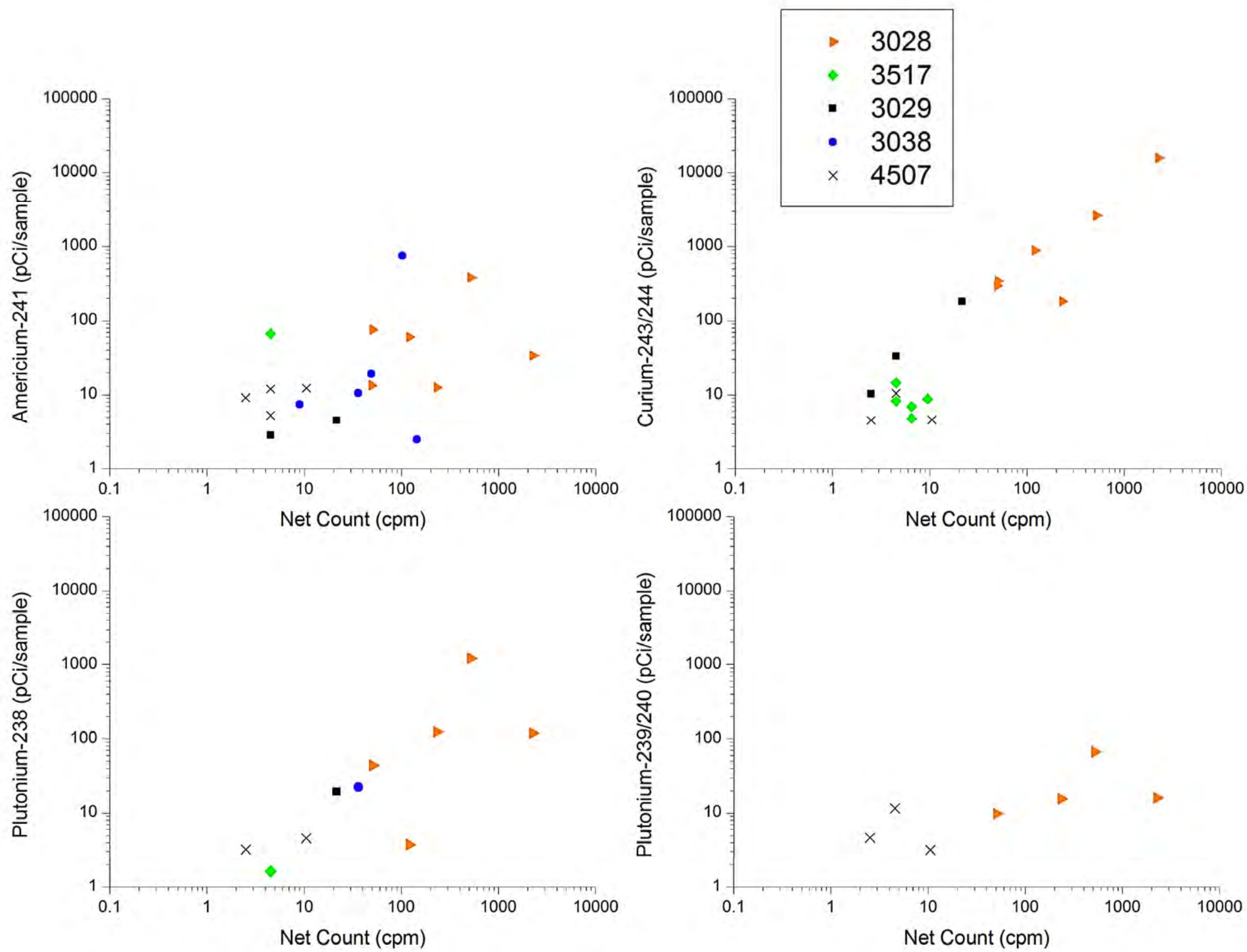

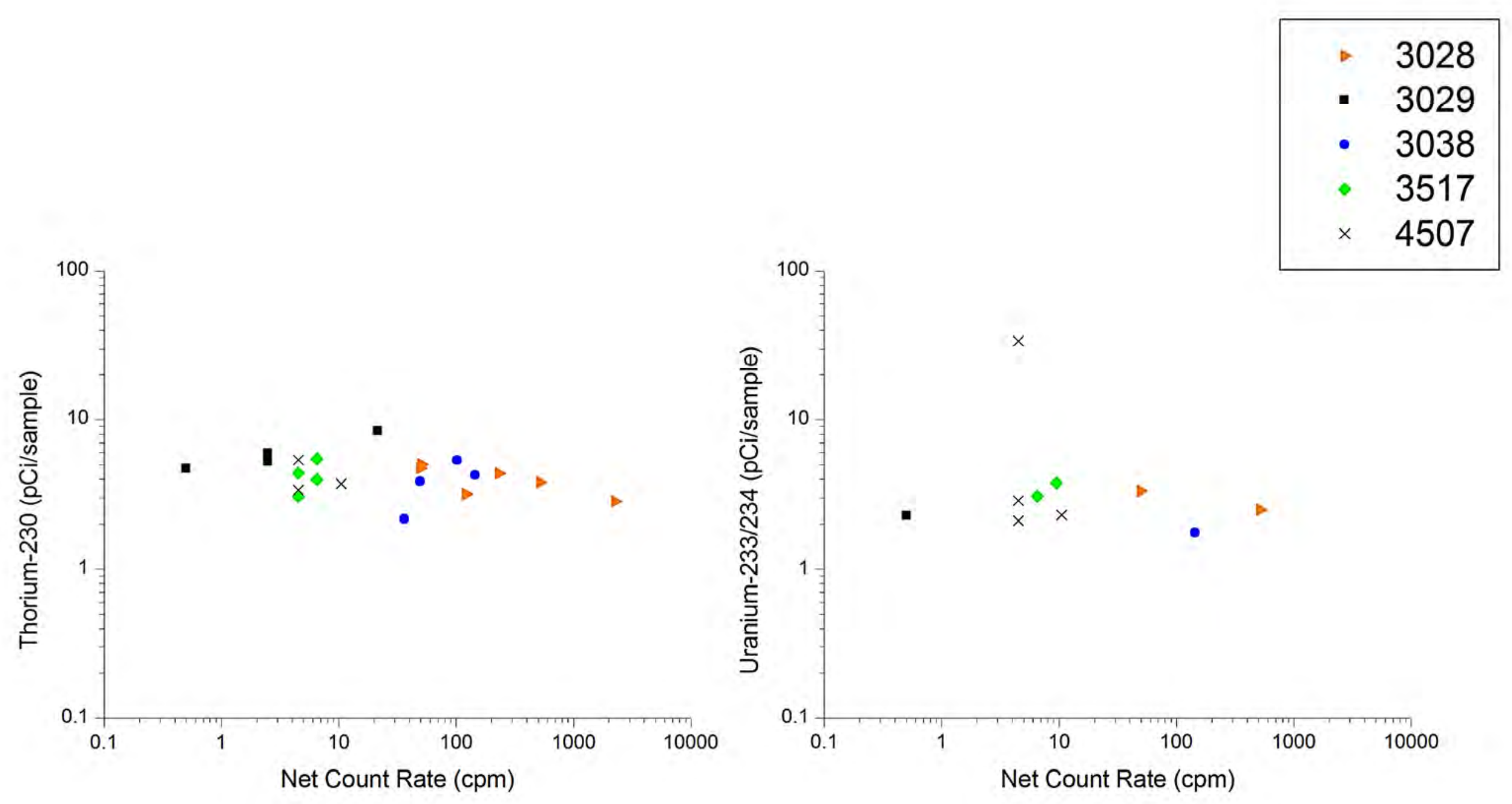

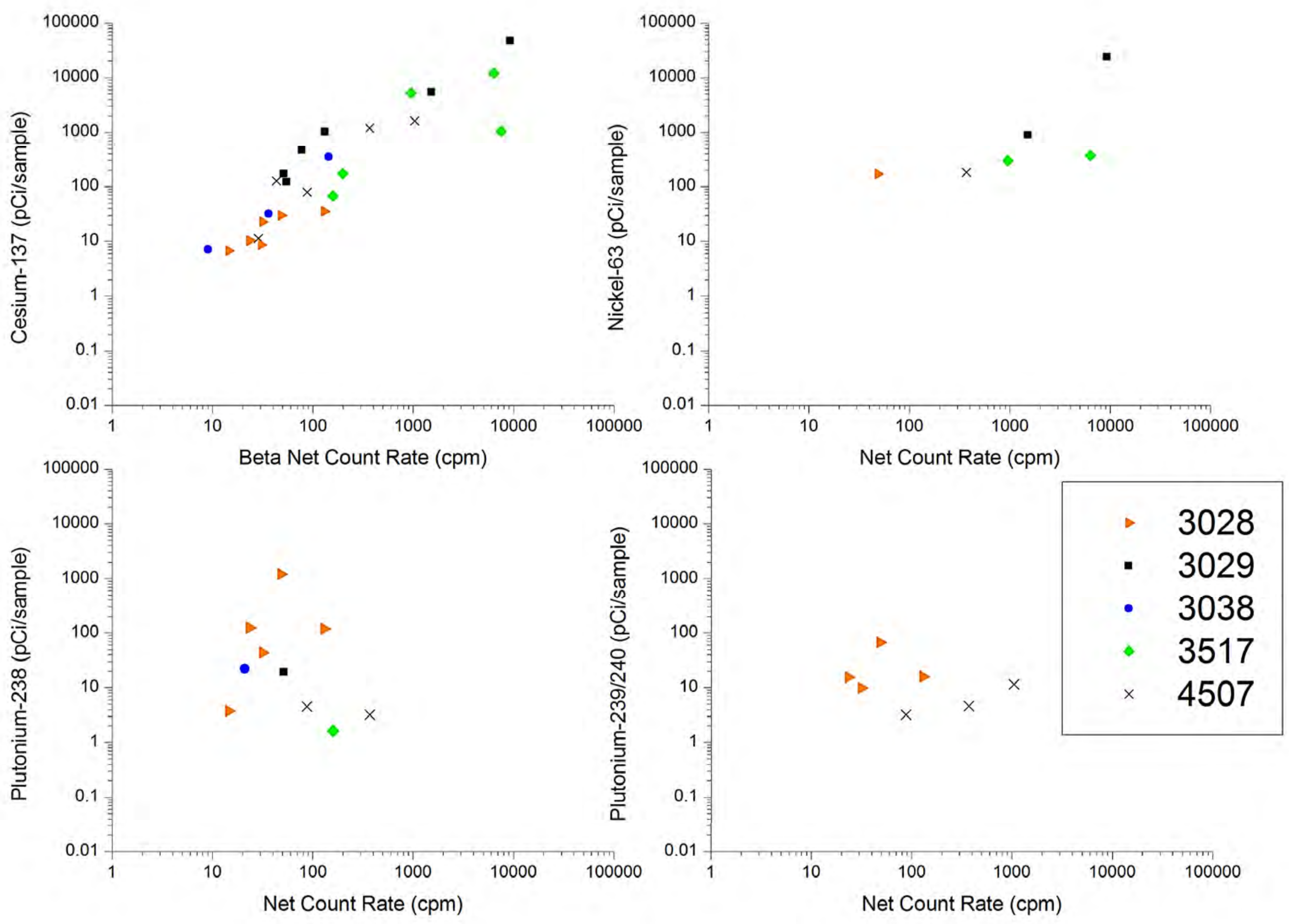

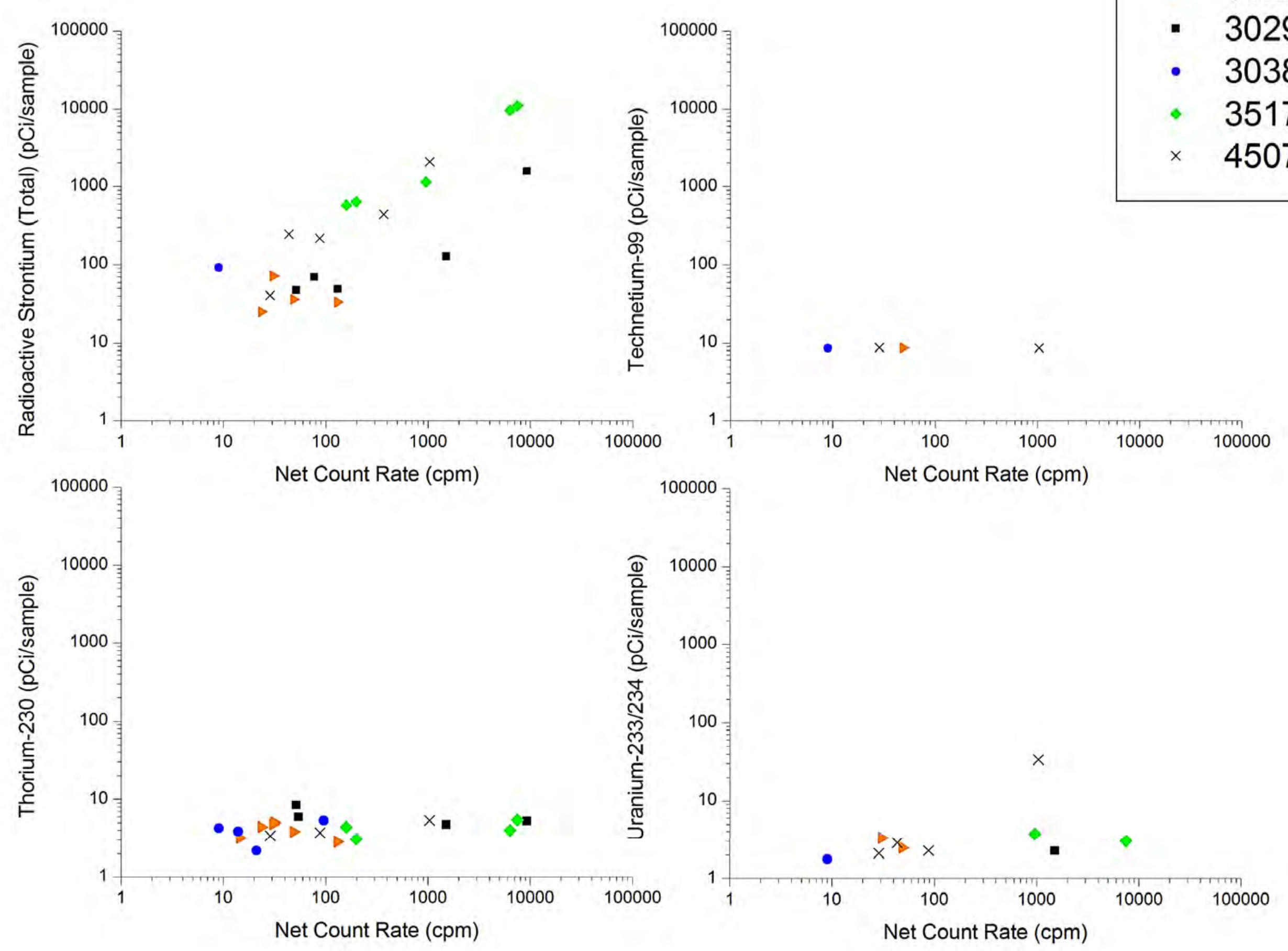
APPENDIX E

LIVING MEMORY SUMMARIES 


\title{
ORAU/ORISE Process Inventory Waste Project ORNL Building 3001
}

\section{LIVING MEMORY SUMMARY}

September 2013

\author{
Linda A. Coffey
}

Waste Management Innovations, Inc.

305 S. Chamberlain Avenue

Rockwood, TN 37854

865-776-5041 


\section{INTRODUCTION}

The following information is derived from interviews of individuals that have personal knowledge pertaining with the Graphite Reactor, Bldg. 3001. This information was collected as part of a process to capture historical insights, usually long after the relevant events occurred. The opinions and comments from interview participants have not been verified, and these statements may contain erroneous or incomplete information.

Discussions were held with four individuals with experience at Bldg. 3001 ranging from 1959 through 2002. One individual was a Chemical Operator, assigned to another facility; two were Facility Managers; and one Operations Supervisor assigned to other facilities. Two individuals are currently employed at the Oak Ridge National Laboratory (ORNL); one individual retired from ORNL in 2008 and continues to supply subcontract support to the ORNL for hot cell and glove box facilities; and one individual retired from the ORNL Isotopes Division in the early 1980s.

\section{INTERVIEWS}

The Chemical Operator stated that in late 1959, plutonium released from an adjacent facility contaminated Bldg. 3001. He and other Chemical Operators, laborers, and maintenance personnel from other facilities were sent to Bldg. 3001 to clean and decontaminate the entire facility. The facility was scrubbed clean from ceiling to floor, everything was wiped down.

One of the Facility Managers served first as the Surveillance and Maintenance Engineer from 1993 to 1995, then as the Facility Manager from 1995 to 2000, and continued to occupy office space there until 2002. The graphite reactor remained internally contaminated. The floor was surveyed and radiological spots were found to be encased in the concrete areas where it was likely that spills occurred during the operational period. The floor was painted to block the activity. Radiological contamination was discovered on the wall below the hatch plates on the back side of the graphite reactor. The walls were cleaned and Plexiglas covers were placed over the hatch plates and sealed with caulk. When the wooden door leading into the hatch room was being replaced, contamination was found in the door jams and framing. The assumption was that the contamination was from the plutonium release that occurred from an adjacent facility in 1959 and contaminated nearby buildings and streets. Historical reports stated that Bldg. 3001 was washed down and repainted. A lot of radiological items were place into the canal. The items were removed and the canal was filled with grout/concrete. The Facility Manager heard a story about a break room sink drain that leaking. When the drain was removed, contamination was found. Mercury was also found in the drain joints. The drain was determined to be an old process line.

The other Facility Manager served from the late 1990s to the early 2000s. There was an underwater canal, about 10-12-ft deep used to transfer the slugs. On one end there was a pit about 20-ft deep. Legacy sources were stored in the canal, as well as radioactive sources from other hot cell facilities that were dumped in there. Because the canal leaked, the sources were removed and the canal was filled with concrete. Other contaminated areas within the facility include the ductwork, the filter house, the reactor pile, and the openings around it.

The Hot Cell Operations Supervisor was responsible for the removal of debris and highly irradiated sources from the canal in 1995. Upon completion, the canal was grouted. 


\section{Record of Communication: Building 3001. Tom Miner}

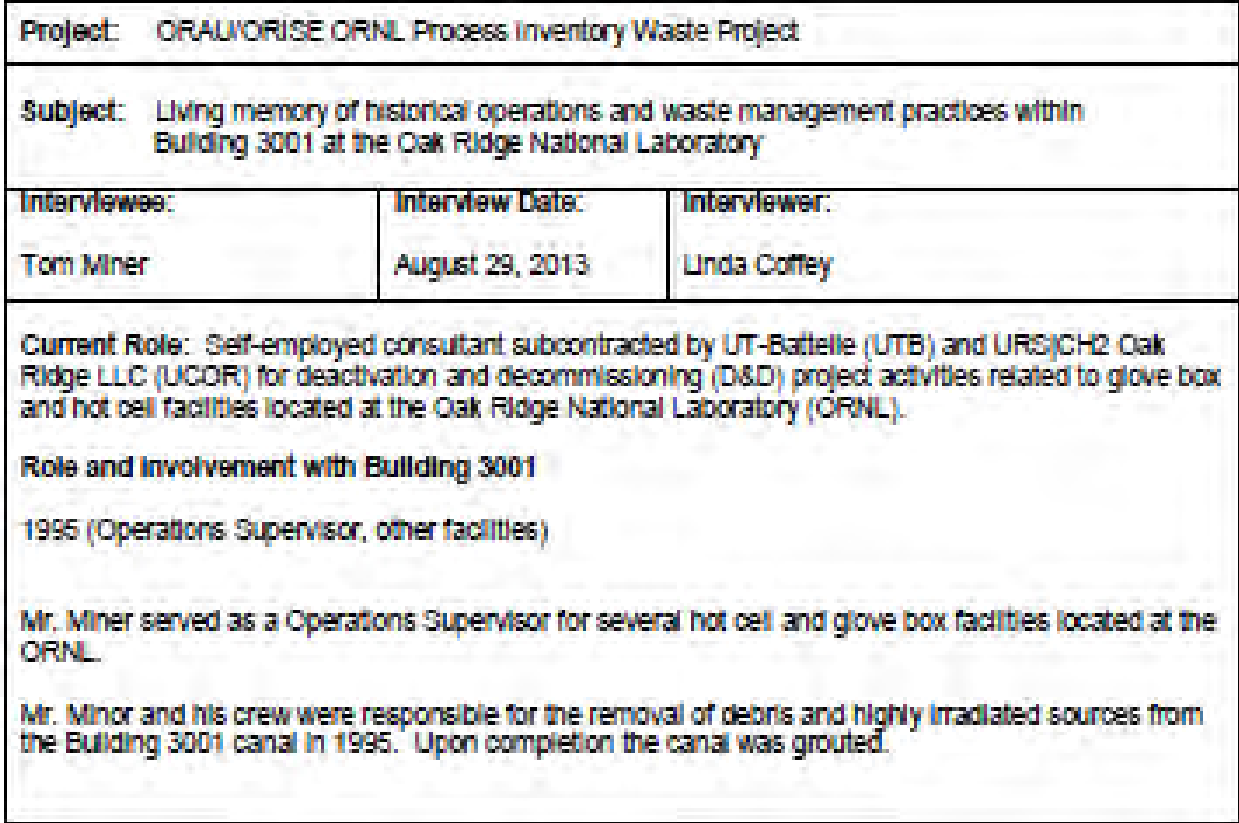

intervewer $\begin{aligned} & \text { Linda } \\ & \text { Coffey }\end{aligned}$


Record of Communication: Building 3001, Mark Ford

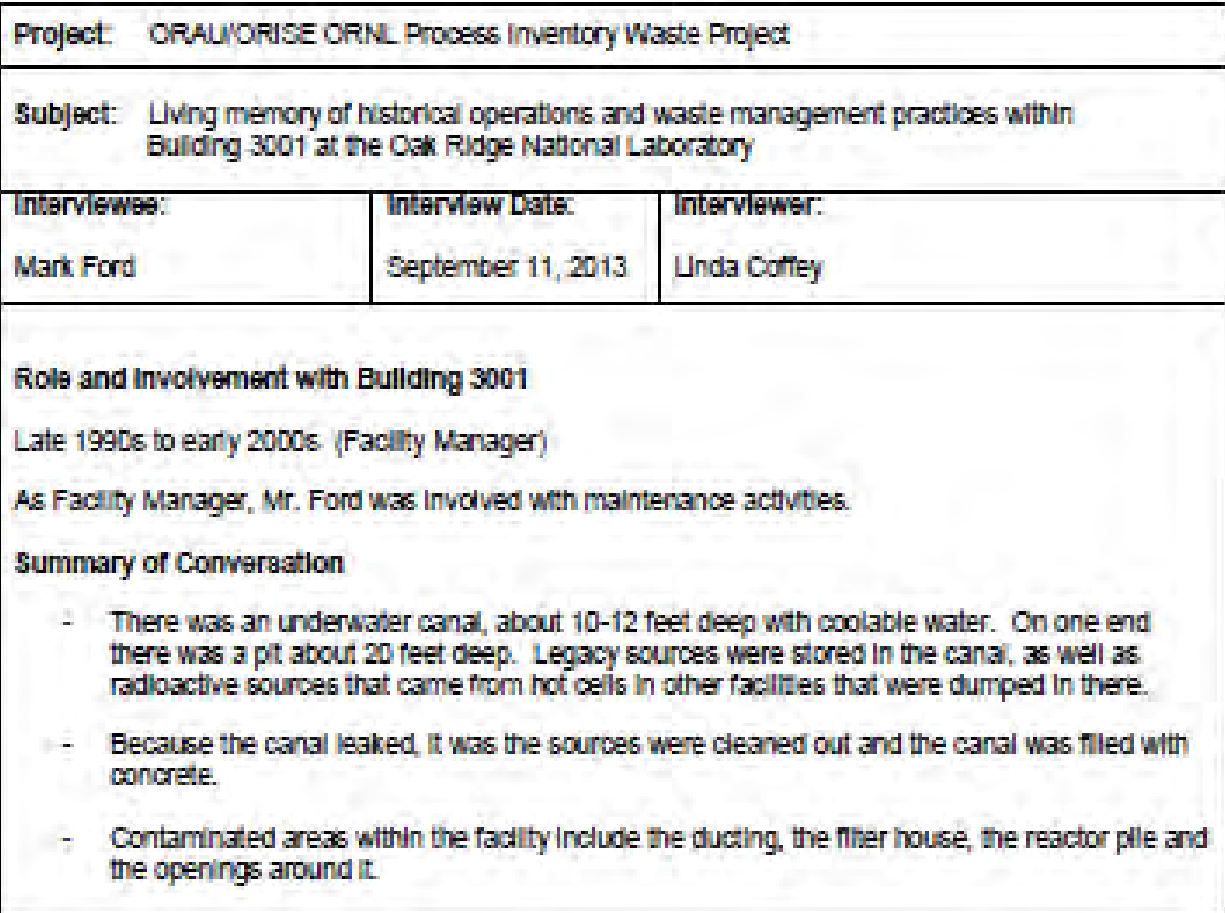

Intervewer. Linda 


\section{Record of Communication: Building 3001, Timothy Cofer}

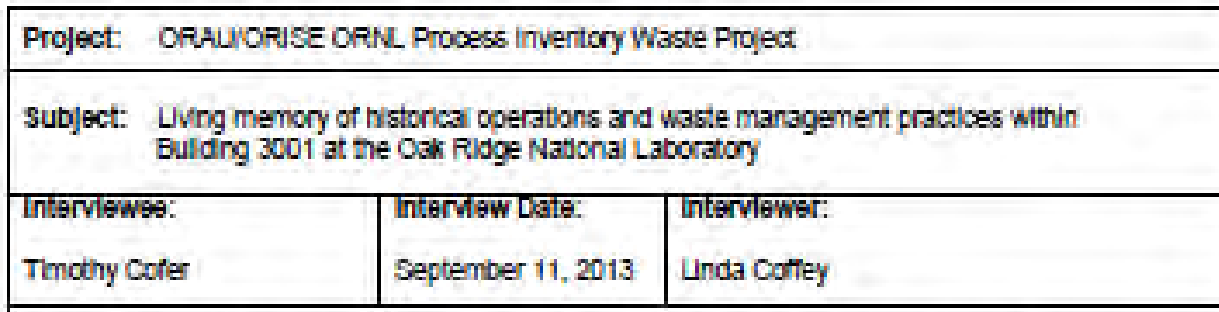

Current Role: Mr. Coter curently has responslblity for the water distrtbuncon plant, the sewer plant. and the storm water colecton system at the Cak Rioge National Laboratory (OFNL).

Role and involvement with Bullding 3001

1993 - 1995 (Survelliance and Maintenance Faclity Engineer)

$1996-2000$ (Facllty Manager)

$2000-2002$ (othoe location)

As the Surveliance and Maintenance (S\&M) Engineer, as wel 36 Facilty Manager, Mr. Cofer was Involved with al survelliance and maintenance activites. In addition, Mr. Cofer's oflice contrived to be located in Eulding 3001. even ater he was no longer responsible for the facilty.

\section{Summary of Converastion}

- There were two sides of the bullding. The graphite reactor side of the bulding w3s a museum. The other side was offce used for offce space.

- In the mid1990s, when the wooden door leadling into the hatch rocm w3s beling replaced, contamination was found in the framing and door jams. The assumpton was Grat the contamination was from the plutonlum relezse that occured from a hot cel in an adlacent faclity that contaminated the neaty buldings and streets. Historical reports stated that Bullding 3001 was washed down and repainted.

- In the mid1990s, radological contamination was found on the wal below the hatch plates on the back side of the graphte reactor. The wals were cleaned and Plexiglas covers were placed over the hatch plates and sealed atth caulic.

- A lot of radological items were placed into the canal. The tems were removed and the cana was tlled win grouticoncrete.

- The graphite reactor remained intemaly contaminated. Whatever was generated during the process was stll gere.

- In the mid1990s, the floor was surveyed and radiologlcal spots were found to be encased in the concrete in areas where it was likely that spills occurred. The foor was painted to biock the actulty.

- Mr. Cofer was told that when the drain connected to a break room sink was being replaced due to leakage, they discovered that it was contaminated. The dran was determined to have been old process ine. Mercury was also found in the drain loints.

intenvewer: Linda 
Record of Communication: Building 3001, Bobby Byrum

Project. ORAuIORIse ORNL. Process inventory waste Project

Subject: Living memory of ivstorical operations and waste management practices within Bulding 3001 at the Oak Ridge National Laboratory

Intarvewe9:

Bobby Byrum

Tnterviewer:

Auguat 15,2013

Unda Cottey

Current Role: Retired from isotopes Division, Oak Ridge National Laboratory (OFNL).

Role and Involvement with Eulding 3001

Late 1959 (Chenical Operator)

Mr. Byrum was working as chemica operator in another facllty when he was sent to assist with decontamination activites inside of Bulding 3001.

summary of Conversation

Mr. Byrum recalled the following informaton related to his experlence while working in Eulding 3001 :

- Chemical operators, laborers, and maintenance persornel from other faclities were sent to Bulliling 3001 to clean and decontaminzte the entre faclity ather plutonlum was released from an adjacent facility.

- The faclity was scrubbed cleaned from celling to foor. Everyting was wiped down, including the bearns and all the iron.

Linda
Coffey 


\section{ORAU/ORISE \\ Process Inventory Waste Project \\ ORNL Building 3005}

\section{LIVING MEMORY SUMMARY}

September 2013

\section{Linda A. Coffey}

Waste Management Innovations, Inc.

305 S. Chamberlain Avenue

Rockwood, TN 37854

865-776-5041 


\section{INTRODUCTION}

The following information is derived from interviews of individuals that have personal knowledge pertaining to the Low-Intensity Testing Reactor, Bldg. 3005. This information was collected as part of a process to capture historical insights, usually long after the relevant events occurred. The opinions and comments from interview participants have not been verified, and these statements may contain erroneous or incomplete information.

Discussions were held with two individuals with experience at Bldg. 3005 ranging from 1993 through 2013. Both individuals have served in the capacity of Facility Manager. Both continue to provide support to ORNL facilities.

\section{INTERVIEWS}

One individual served as the Surveillance and Maintenance Facility Manager from 1993 to 1995, then as the Facility Manager from 1995-2000. He stated that the beryllium shielding was contained within the reactor. Lead paint flakes off in the high bay area. Until recent years, Health Physics personnel occupied one part of the facility. Plant and Equipment personnel occupied the other side.

The other individual has served as the current Facility Manager since 2000. He stated that decommissioning and decontamination (D\&D) work was completed earlier this month in the Annex, located in the northwest portion of the building. The trench located on the northwest side is contaminated with daughter products of reactor fuels. There is contamination in the control room area located on the 3rd floor in the southern part of the building. The reactor area on the upper floors is a radiological buffer area. The facility has electrical power, steam, and an active dry pipe sprinkler system for fire protection. The bottom floor on the west side currently is used for storage. The east side is vacant. The east side low roof is deteriorating and leaks. Mold and mildew is rampant in the east side of the building. The reactor

ventilation system has been isolated. Other contaminated areas within the facility include the ducting, the filter house, the reactor pile and the openings around it. The Preliminary Hazard Screening (PHS) report and the Safety Authorization Basis (SAB) document are available for additional information. 


\section{Record of Communication: Building 3005, Timothy Cofer}

Project. ORAUIORISE ORNL. Process inventory Waste Project

Subject: Living memory of historical operations and waste management practices within Bulding 3005 at the Oak Ridge Nattonal Laboratory

\begin{tabular}{|l|l|l|}
\hline Thtervlewe9: & Intervaw Date: & Interviewer: \\
Timothy Coter & September 11,2013 & Unda Cotity \\
\hline
\end{tabular}

Current Riole: Mr. Cofer curently has responstbilty for the water olstribution plant, the sewer plant, and the storm collecton system Oak Fudge Nationai Laboratory (OFNL).

Role and Involvement with Bullding 3005

1993 - 1995 (Survelliance and Mantenance Facitiy Engineer)

1995 - 2000 (Faclity Manager)

Summary of Conversation

Mr. Coter recalled the following information related to his experience wth Bulding 3005 :

- Beryllum stielding was contained within the reactor.

- Lead paint fakes ofr in the high bay area.

- Unti recent years, Heath Physics persornel occupled one part of the facilty. Plart and Equipment personnel occupled the other side.

Intervewer. Linda A,
Coffey 
Record of Communication: Building 3005, Bruce Howard

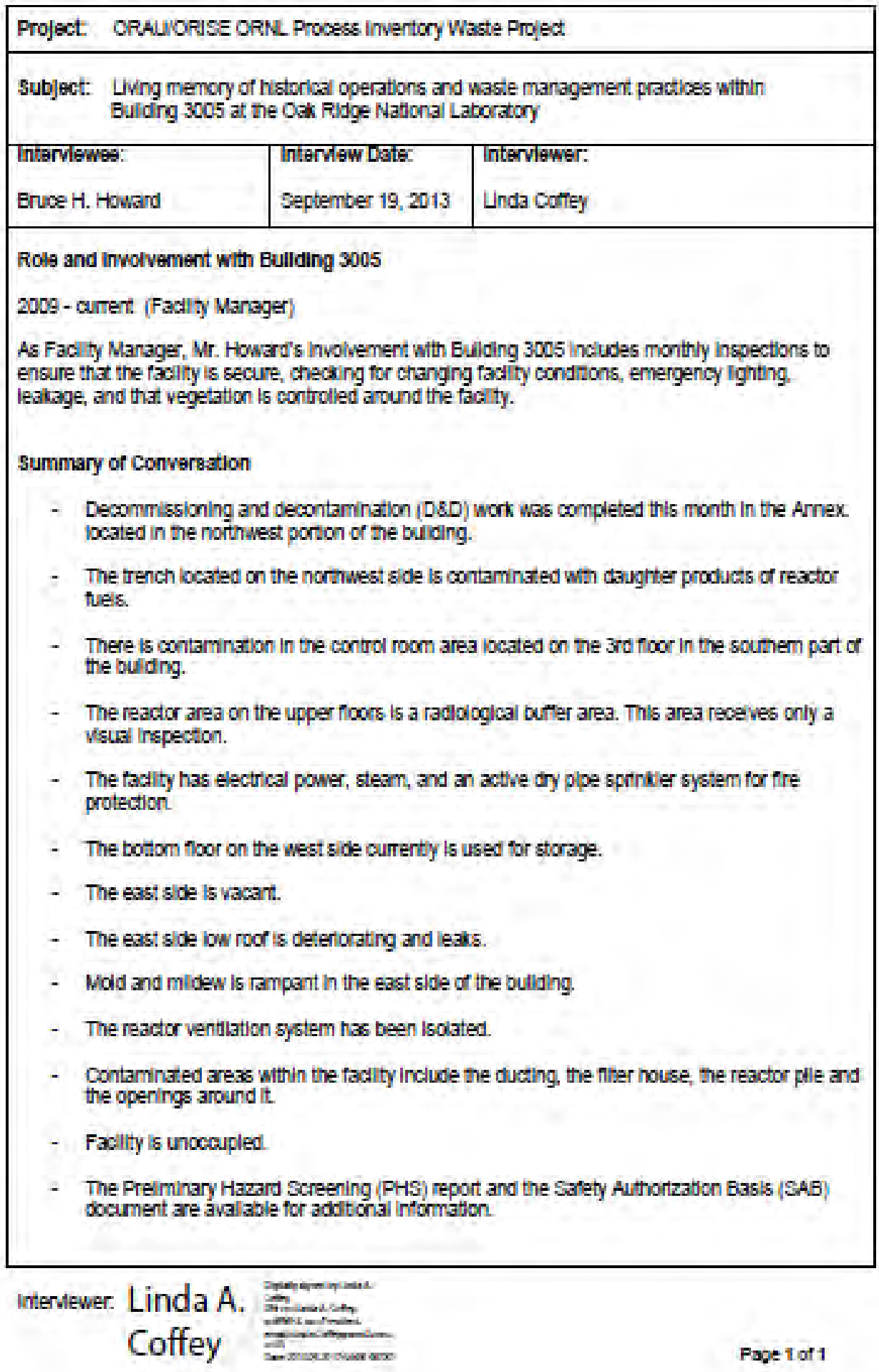




\title{
ORAU/ORISE \\ Process Inventory Waste Project ORNL Building 3010
}

\section{LIVING MEMORY SUMMARY}

September 2013

\author{
Linda A. Coffey
}

Waste Management Innovations, Inc.

305 S. Chamberlain Avenue

Rockwood, TN 37854

865-776-5041 


\section{INTRODUCTION}

The following information is derived from interviews of individuals that have personal knowledge pertaining to the Bulk Shielding Reactor Facility, Building 3010. This information was collected as part of a process to capture historical insights, usually long after the relevant events occurred. The opinions

and comments from interview participants have not been verified, and these statements may contain erroneous or incomplete information.

Discussions were held with two individuals with experience at Building 3010 ranging from 1984 through 2000. Both individuals were Facility Managers and both are currently employed at the Oak Ridge National Laboratory (ORNL).

\section{INTERVIEWS}

One Facility Manager served while the facility was operational from 1984 - 1986. He stated that the Bulk Shielding Reactor had a unique capability with a core that could be moved around in a pool and irradiate experiments while they sat stationary in different parts of the pool. In another area of the pool was the Pool Critical Assembly where Nuclear Engineering Departments from universities would conduct experiments.

The other Facility Manager served from late 1994 - 2000. He stated that the pool reactor had an aluminum liner between the concrete super structure and the walls. The 100,000 gallon pool was bled out from the bottom and replaced with approximately five gallons of demineralized water a minute to minimize corrosion. The control rods were removed, deactivated, and placed in B25 boxes that were encased in concrete for disposal. Most people that were involved with legacy operations in this facility have retired and many have passed away.

Neither of these two individuals recalled or heard stories of contamination events that occurred in this facility. 
Record of Communication: Building 3010, Mark Ford

Project: ORAUIORISE ORN. Process invertory Waste Project

Subject: Liveng memory of historicai coerations and waste management practices within Bulding 3010 at the Oak Ridge National Laboratory

\begin{tabular}{l|l|l}
\hline Intervewe9: & Intervew Date: & Intervewer: \\
Mark Ford & Septenber 11, 2013 & Unda Coffey \\
\hline
\end{tabular}

Role and involvement with Bullding 3010

1984 - 1986 (Operations Facilty Manager)

Mr. Ford was one of the Operations Faclity Managers whlle the facilty was operation.

Summary of Conversation

- The Buk Stielding Reactor had a unique capabilty with a core that could be moved around in a pooi and irradisfe experiments whle eney sat statonary in diferent parts of the poo.

- There was also another area in the pooi caled the Pool Crtica Assembly shere nuciear engineenng departments from universiltes would come in and conduct experiments.

Linda
Intendewer 
Record of Communication: Building 3010, Timothy Cofer

Project: ORAUIORISE ORNL. Process inventory Waste Project

Subject: Living memory of ivstorical operations and waste management practices within Bulling 3010 at the Oak Ridge National Laboratory

\begin{tabular}{|c|c|c|}
\hline Thiarvewe9: & Intervaw Date: & Intervewer: \\
\hline Timothy coter & September 11,2013 & Unda Cott: \\
\hline
\end{tabular}

Current Riole: Mr. Cofer curently has responsiblity for the water distriburon plant, the sewer plant, and the water storm colection system at the Oak Ridge National Laboratory (ORWL).

Role and involvement with Bullding 3010

1994 - 1995 (Maintenance Faclity Engineer)

1995 - 2000 (Faclity Manager)

As the Survellance and Maintenance (SSM) Engineer, $\mathbf{x}$ wel 36 Facllity Manager, Mr. Cofer was involved whin all survellance and maintenance activites.

Summary of Conversation

- The Euk Stielding Reactor was a pool reactor system. The pool had an aluminum iner between the concrete super structure and the walls.

- The 100,000 gallon poo w3s bied out from the botom and repiaced with approximately five galions of demineralzed water a minute to minimize corrosion.

- The contrd rods were removed, deactivated, and placed in B25 boxes that were encased in concrete for dsposa.

- Most people that were involved wth legacy coerations of this facity have retired and many have passed away.

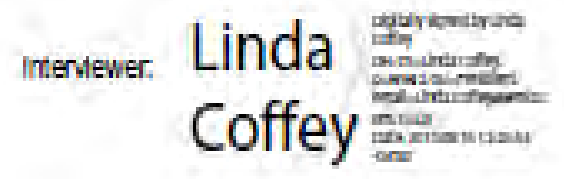




\section{ORAU/ORISE \\ Process Inventory Waste Project ORNL Building 3028}

\section{LIVING MEMORY SUMMARY}

September 2013

\section{Linda A. Coffey}

Waste Management Innovations, Inc.

305 S. Chamberlain Avenue

Rockwood, TN 37854

865-776-5041 


\section{INTRODUCTION}

The following information is derived from interviews of individuals that have personal knowledge pertaining to Bldg. 3028. This information was collected as part of a process to capture historical insights, usually long after the relevant events occurred. The opinions and comments from interview participants have not been verified, and these statements may contain erroneous or incomplete information.

Discussions were held with four individuals with experience in Building 3028 ranging from 1954 through present day. One was a Group Leader in the Isotopes Department, Operations Division between 1954 and 1988; two were Chemical Operators, promoted to Operations Supervisors ranging from 1975 to 2008; and one was an Isotopes Technician. One individual is currently employed at the Oak Ridge National Laboratory (ORNL), one retired individual continues to supply subcontract support to the ORNL for hot cell and glove box facilities, and two individuals retired from the Isotopes Operations Division in 1980s.

\section{INTERVIEWS}

One retiree worked in several of the Isotopes Facilities during his forty-one year career as the Group Leader in the Isotopes Department, Operations Division. He was also responsible for the decommissioning and decontamination (D\&D) of fourteen facilities, as well as the lead author of the ORNL Final D\&D Report for Bldg. 3028. In the early years of his career he conducted shielding calculations for multiple processes. He was involved in the reactor production of isotopes, including the target materials, as well as research and development work to resolve transportation problems. He stated that the facility consisted of two cell blocks. One was a water shielded alpha handling facility where a substantial amount of curium-242, curium-244, and americium-241 work was done. The other block was an iodine-131 processing facility. Both cell blocks were decommissioned and decontaminated in the late 1990s. Contamination was reduced to acceptable levels where they could have been taken apart, size reduced, and destroyed at that time. The central part of the building was four stories in order to accommodate the ion-exchange column for the separation of promethium-147. The Xenon facility operated from the early 1960s through 1980. Smear results, radiation levels, operations history, drawings, and drawing lists can be found in the ORNL Building 3028 D\&D Final Report.

One current employee served as Chemical Operator, then as an Operations Supervisor for Bldg. 3028, and was also involved in the decontamination and facility stabilization in the early 1990s. Short-life isotopes were processed on the west end of the building and the contamination in that area of the facility should be minimal. The alpha handling curium cells were all highly contaminated, including the back side of the cells and the crawl space under the cells. Curium levels were so high under the cells that they used three layers of coveralls in order to apply yellow strippable (peelable) paint to the crawl space under the cells. Two people had uptakes from that event. The pit behind the cell operating area was very contaminated where the valves discharged into the tanks. The upper floors were utilized for mice research and contamination levels were low there. At one time there was an attempt to dig out one of the drain lines between Bldgs. 3028 and 3029. They dug down ten feet and were getting 300R readings. The hole was too contaminated to deal with, so they filled the hole up.

One former employee served as a Chemical Operator/Operations Supervisor before retiring from UTBattelle LLC (UTB) in 2008 and continues to supply subcontract support for D\&D activities in Bldg. 3028. He stated that the operating areas around Cells 1-5 were highly contaminated. There were numerous releases in the back of these cells. Curium-244 easily gets airborne and it was difficult to contain. The entire area was contaminated from the ceiling to floor, from the operating area to the charging area in the east wing. Residual quantities of curium remained in the hot cells from legacy work. 
Routine operations were shut down prior to 1985. Activities since that time included ongoing facility maintenance, surveillance and maintenance and cleanout of the curium hot cells in mid 1990s.

One retired individual served as a Radioisotopes Target Technician in the Isotopes Facilities. During the 1970s he worked with tritium targets on the third floor of Bldg. 3028. He stated that residual tritium contamination would be gone by now. He recalls that a lot of curium work was done in Bldg. 3028. They had to place the pellet on a copper block and ran cold water through it to keep it from getting too hot.

Record of Communication: Building 3028, Tom Miner

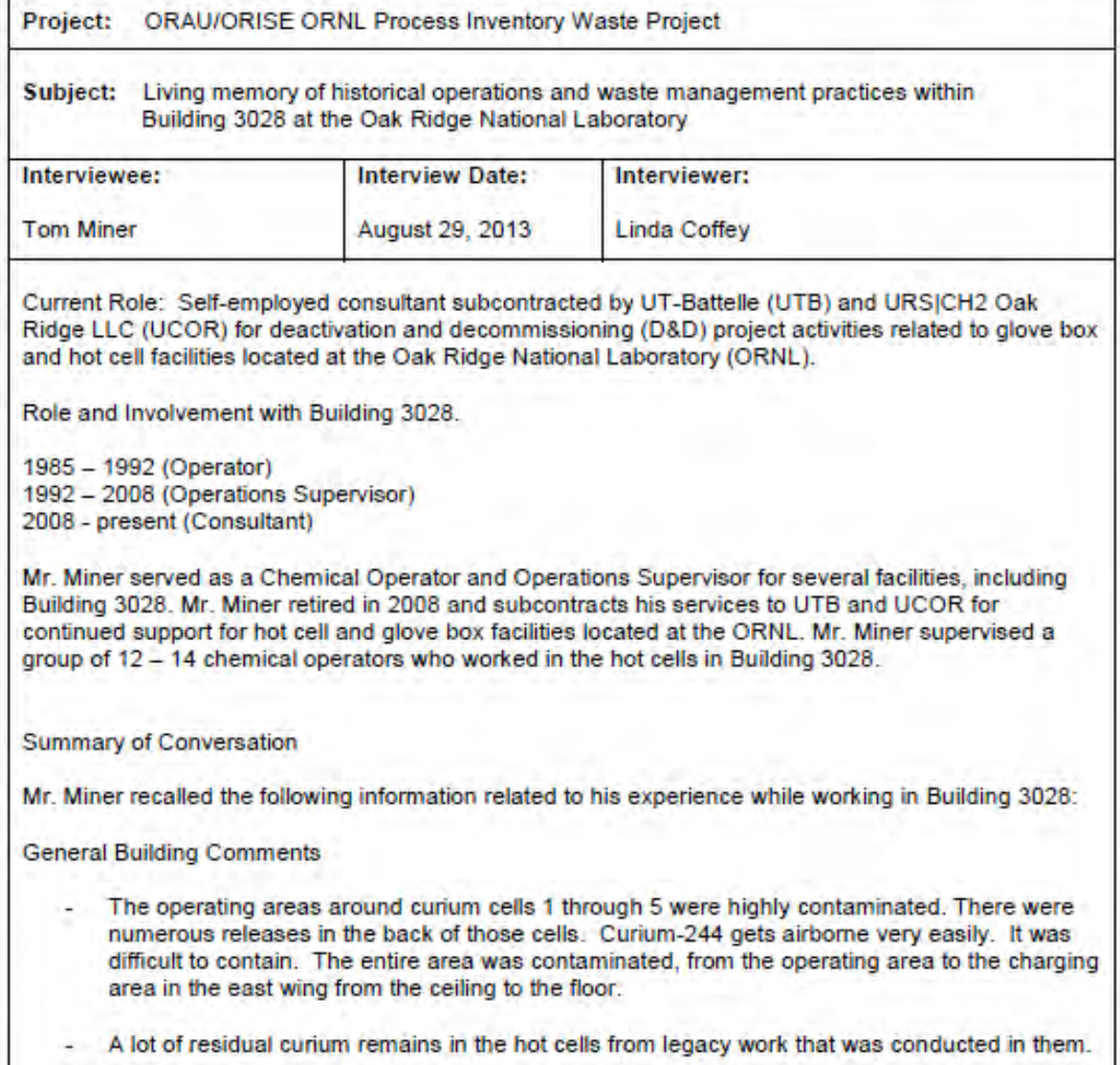

- The operating areas around curium cells 1 through 5 were highly contaminated. There were numerous releases in the back of those cells. Curium-244 gets airborne very easily. It was difficult to contain. The entire area was contaminated, from the operating area to the charging area in the east wing from the ceiling to the floor.

- A lot of residual curium remains in the hot cells from legacy work that was conducted in them.

Mr. Miner was interviewed by Acceptable Knowledge Expert Tom Krause on May 18, 2005 related to "Historical operations and waste management at Building 3028." The following report represents Mr. Miner's responses during that interview (Tracking Document C097, TRU/Alpha Low Level Waste Treatment Project, TRU-OPS-1110, Rev. B.).

Note: Mr. Miner has been involved with Buildings 3028, 3038, 3517, 3026D, 3029, 3505, 4507,3042 (ORR), and 3010 (BSR).

What is your connection with Building 3028 ? -Or- What was your position at Building 3028 ? Mr. Miner was an operator from 1985 to 1992 and has been the Operations Supervisor from 1992 to present.

During what time frame were you involved with operations at Building 3028 ? 1985 to present. 


\title{
Record of Communication: Building 3028, Tom Miner
}

\author{
What programs or projects were conducted in this building? \\ Routine operations in this building were shut down prior to 1985. Activities since that time included
} ongoing facility maintenance, SNM maintenance and cleanout of the Curium hot cells in 1996-97.

What is your recollection of waste management practices at this building?

Waste from this building was managed in accordance with ORNL wide procedures in place at the time.

Are you familiar with how waste from this building was packaged? If yes, can you provide details (e.g., layers of confinement, filters on drum or inner packages, rigid drums liners)?

Individual items were double-bagged and placed in stainless steel drums with another bag liner used to hold all waste within a drum. No liquids, powders, or sealed containers were placed in the drums.

Was waste segregated at this building? If yes, how?

Waste was not segregated as all the hot cells were interconnected.

Is there anyone else that may be able to help with this information? No one else still available."

\begin{tabular}{|c|c|}
\hline terviewer: & 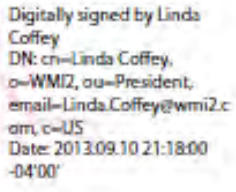 \\
\hline
\end{tabular}




\section{Record of Communication: Building 3028, David Drake}

\section{Project. ORAuIORISE ORNL. Process inventory Waste Project}

Subject: Living memory of historical operations and waste management practices within Bulding 3028 at the Oak Ridge National Laboratory

\begin{tabular}{|l|l|l|}
\hline Intarvewe9: & Intervew Date: & Interviewer: \\
David Drake & Augukt 15,2013 & Unda Coftey \\
\hline
\end{tabular}

Current Role: Group Leader for Nuclear Research Support and Nuclear Sclence and Engineering Directorate at UT-Eathele (UTB) located at the Oak Roge National Laboratory (OFNL).

Role and Invovement win Bulding 3028

1975 - 1985 (Chemical Operator)

1985 - 1991 (Operations Supervisor)

Mr. Drake whlle serving as a chemical operator and as an operations supervisor between 1975 and 1991, Mr. Drake worked in several, incuding Eulding 3028.

\section{Summary of Conversation}

Mr. Drake recaled the folowing information related to tis experlence while working in Bulding 3028 :

- Short life isotopes were processed on the west end of the bulding in the Short-Lved flssion Product Facilty, contaminaton issues should be minimal in that area.

- The alpha handing curtum cels were all highly contaminated, including the back side of the cels, and the cram space under the cells. Cunum levels were so high under the cals that they triple dressed in order to apply yellow strippable (peelable) paint to the crasl space under the cells. Two pecple had uptakes from that event.

- The pit behind the cell operating area was very contaminated where the valves discharged into the tanks.

- The upper foors were pretty much a mice research work area, so contamination levels were low there.

- There was an attempt to dg out one of the drain ines between 3028 and 3029 . They went down ten feet $\left(10^{\prime}\right)$ and were getting 300 r readings. So they nlled the hoie up and waked away. It was just too contaminated to deal with.

- Mr. Drake was also involved in decontamination and facilty stabilzation hn the early 19906.

intervewer.

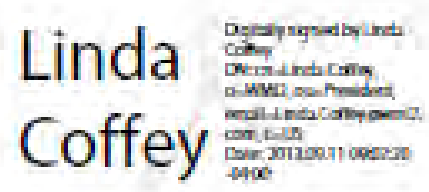




\begin{tabular}{|c|c|c|c|}
\hline \multicolumn{4}{|c|}{ Project: ORALIORISE ORRL Process inventory waste Project } \\
\hline Subjec: & \multicolumn{3}{|c|}{$\begin{array}{l}\text { Living memory of historical operations and waste management practices within } \\
\text { Bulding } 3028 \text { at the Oak Ridge National Laboratory }\end{array}$} \\
\hline \multirow{2}{*}{\multicolumn{2}{|c|}{$\begin{array}{l}\text { Tntarvew9: } \\
\text { Kart Hat }\end{array}$}} & Intervaw Date: & Intervewer: \\
\hline & & September 3,2013 & Unda Cottey \\
\hline \multicolumn{4}{|c|}{ Current Role: Retired, ORNL isotopes Division } \\
\hline \multicolumn{4}{|c|}{ Role and invoivement win Eulding 3028} \\
\hline \multicolumn{4}{|c|}{$\begin{array}{l}1954 \text { (Co-0p Student) } \\
1959-1988 \text { (isotopes Opersilion Division, Group Leader }\end{array}$} \\
\hline \multicolumn{4}{|c|}{$\begin{array}{l}\text { Mr. Hat worked in several of the isctopes Facilties during his } 41 \text { year career. In the earty years of his } \\
\text { career he concucted shielding caiculations for multple processes and he was very Involved in the } \\
\text { reactor producton of Isotopes and caiculating the target materials required, as well } 36 \text { research and } \\
\text { development work to resove transportation problems. }\end{array}$} \\
\hline \multicolumn{4}{|c|}{$\begin{array}{l}\text { Mr. Hart became involwed with Bulding } 3028 \text { in the mid } 1980 \text { 's. He was responsible for } \\
\text { deconmissioning and decontamination (DSD) for fourteen faciltes, Including Bulding } 3028 \text {. }\end{array}$} \\
\hline \multicolumn{4}{|c|}{ Summary of Converaation } \\
\hline \multicolumn{4}{|c|}{ Mr. Hatf stated the following information Bullding 3026 : } \\
\hline & \multicolumn{3}{|c|}{$\begin{array}{l}\text { The faclity consisted of two cell biocks. One was a water shlelded aipha handing facilty } \\
\text { where a lot of curlum-242, curlum-244, and americum-241 work were done. The other cell } \\
\text { block was an lodine-131 processing facily. }\end{array}$} \\
\hline - & \multicolumn{3}{|c|}{$\begin{array}{l}\text { Both cell blocks were decommissioned and decontaminated in the late } 19905 \text {. Contaminaton } \\
\text { was reduced to acceptable leveis where they could have been taken apart and destroyed at } \\
\text { that time. }\end{array}$} \\
\hline & \multicolumn{3}{|c|}{$\begin{array}{l}\text { The central part of the bullaing is four stores in order to accommodate the lon-exchange } \\
\text { column for the separation of promethum- } 147 \text {. Various sundry actitles took place on al the } \\
\text { levels of that buliding. }\end{array}$} \\
\hline- & \multicolumn{3}{|c|}{ Xenon- 133 facily operated from the earty 1960 s trrough 1960 . } \\
\hline- & \multicolumn{3}{|c|}{$\begin{array}{l}\text { Wr. Hatl was the lead aumor of the DSD reports for Bulding } 3028 \text {. Smear resuls, radiation } \\
\text { levels, operations history, drawngs, and drawting lists can be found in the Final Bullding } 3028 \\
\text { DSD report. }\end{array}$} \\
\hline
\end{tabular}

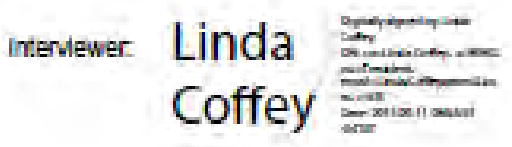




\section{Record of Communication: Building 3028, Bobby Byrum}

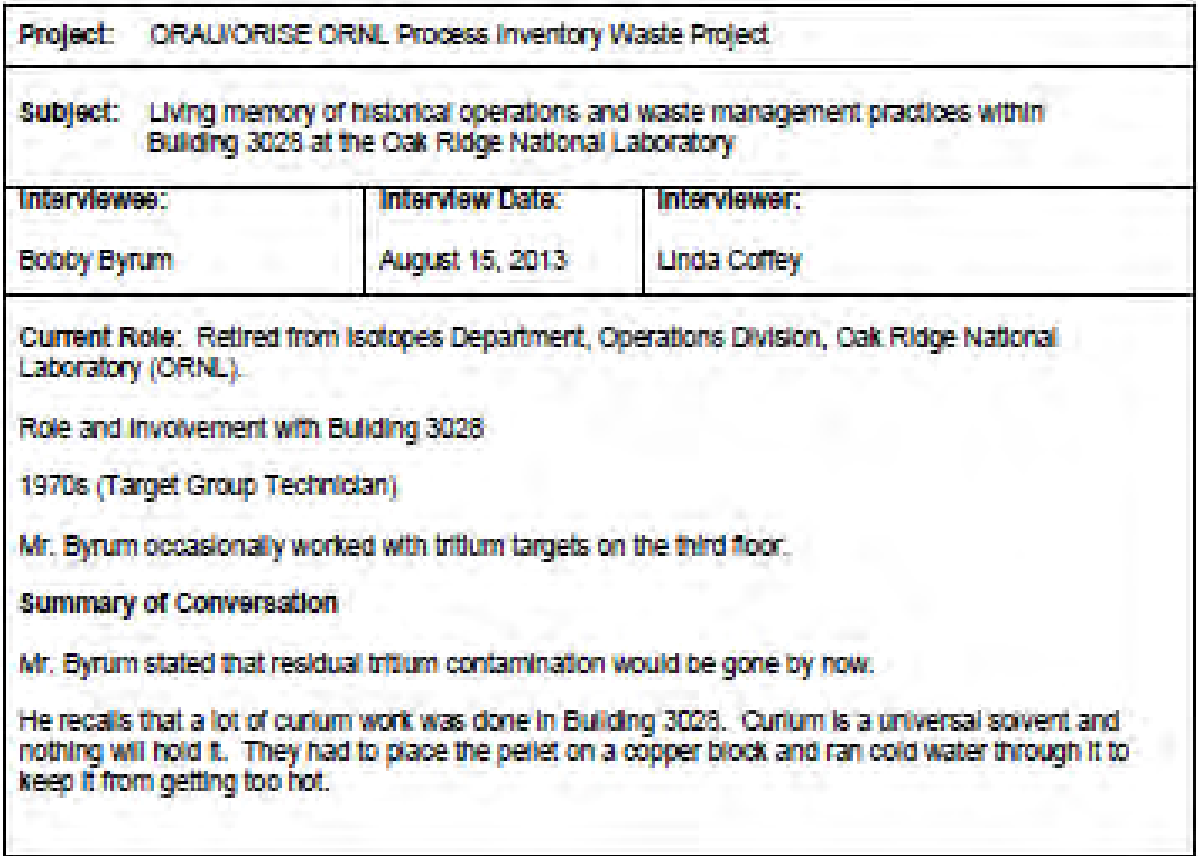

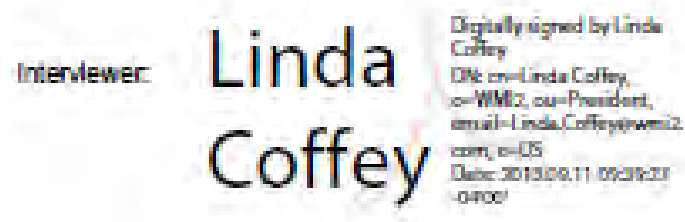




\section{ORAU/ORISE \\ Process Inventory Waste Project ORNL Building 3029}

\section{LIVING MEMORY SUMMARY}

September 2013

\section{Linda A. Coffey}

Waste Management Innovations, Inc.

305 S. Chamberlain Avenue

Rockwood, TN 37854

865-776-5041 
The following information is derived from interviews of individuals that have personal knowledge pertaining to the Source Development Laboratory, Bldg. 3029. This information was collected as part of a process to capture historical insights, usually long after the relevant events occurred. The opinions and comments from interview participants have not been verified, and these statements may contain erroneous or incomplete information.

Discussions were held with three individuals with experience in Bldg. 3029 ranging from 1954 through present day. Two were Chemical Operators, promoted to Operations Supervisors between 1975 to present; and one was the Group Leader in the Isotopes Operations Division. One individual is currently employed at the Oak Ridge National Laboratory (ORNL), one continues to supply subcontract support to the ORNL, and one individual retired from ORNL in 1988.

One former employee served as a Chemical Operator/Operations Supervisor before retiring from UT-Battelle in 2008 and continues to supply subcontract support for decontamination and decommissioning (D\&D) activities in Bldg. 3029. He stated that all the cobalt sources were removed from the Co-60 Garden, except for two. Two were so badly degraded that they could not be removed. The hot cells and charging stations were highly contaminated, especially Cells 1, 2, and 3. Other areas of contamination include the glove boxes, drains, and vent pipes.

The other Chemical Operator/Operations Supervisor worked in the facility from 1975-1991. He stated that the grass and dirt in the area of the west end had continuously high levels of contamination. In the late 1980s, the area was covered with concrete. The ground area around the remainder of the building should be suspect for contamination. Alpha contamination was discovered while repairs were being made to the two fume hoods. There was never a record of alpha being in the facility. However, from the 1940s through the 1970s, a lot of historical records were lost. The most common remedy was to paint over contamination. Non-surface areas were suspect for all types of contamination.

The Isotopes Operations Division Group Leader was the lead author of the final decontamination and decommissioning (D\&D) report for Bldg. 3029. He stated that this report contains smear results, radiation levels, operations history, drawings, and drawing lists. 
Record of Communication: Building 3029, David Drake

Project: ORALIORJSE ORNL Process inventory Waste Project

Subject: Living memory of historica operations and waste management practices within Bulding 3029 at the Ock Ridge Natlonal Laboratory

\begin{tabular}{|c|c|c|}
\hline Intervlewค9: & Intervew Date: & Intrivewer: \\
\hline David Drake & August 15,2013 & Unda Colley \\
\hline
\end{tabular}

Current Role: Group Leader for Nuclear Research Support and Nuclear Sclence and Engneening Directorate at UT-Eathele (UTB) located at the Oak Ridge National Laboratory (ORNL).

Role and invovement whe Eulding 3029

1975 - 1985 (Chemicai Operator)

1985 - 1991 (Operations Supervisor)

Mr. Drake while serving $a$ a chenical operator and as an operations supervisor between 1975 and 1991 , Mr. Drake worked in several, incuding Euling 3029.

\section{Summary of Conversation}

Mr. Drake recaled the foliowing information related to his experlence whlle working in Bulding 3029 :

- The grass ard dit in the area of the west end had conthuously high leveis of contamination. In the late 1980s, the area was covered with concrete. The ground area around the bulding should be suspect for contamination.

- Aipha contamination was dscovered whle repairs were beling made to two fume hoock. There wasnit a record of aipha ever being in that faclity. However, in the $19405-1970$ s a lot of historical records were lost and the way programs changed during that time non-surface areas were suspect for all types of contamination. The most common remedy was to paint over it.

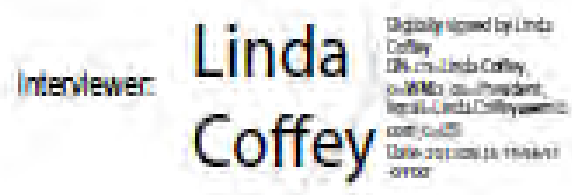




\section{Record of Communication: Building 3029, Karl Haff}

Project ORALIORISE ORNL Process inventory Waste Project

Subject: Living memory of historical operations and waste management practices within Bulding 3029 at the Oak Ridge National Laboratory

\begin{tabular}{|l|l|l}
\hline Intervew99: & $\begin{array}{l}\text { Intervew Date: } \\
\text { Karl Hatt }\end{array}$ & Interviewer: \\
September 3, 2013 & unda Cottey
\end{tabular}

Current Role: Retired, ORNL isotopes Division

Role and Invovement win Eulding 3029

1954 (Co- 00 Student)

1959 - 1983 (isotopes Operstion Division, Group Leader)

Mr. Hart worked in several of the isotopes Facitles during his 41 year career. In the early years of his career he conducted shleiding calculations for multple processes and he was very involved in the reactor producton of isotopes and calculating the target materlals required, as wel as research and development work to resoive transportation problems.

Mr. Hart became involved wth Bulding 3029 in the mid 1980's. He was responsible for decommissioning and decontamination (DSD) for fourteen faciltes, including Bulding 3029.

Summary of Conversation

Mr. Hart stated the following information Buliding 3029:

- Mr. Hatr was the lead aunor of the DSD reports for Bulding 3029. Smear result, radation levels, operations history, drawngs, and drawing lists can be found in the Final Bullding 3029 DBD report.

Intervewer:

Linda Coffey $=$ 


\section{Record of Communication: Building 3029, Tom Miner}

Project: ORAMIORISE ORNL. Process inventory Waste Project

Subject: Living memory of historica operations and waste management practices within Bulding 3029 at the Oak Ridge National Laboratory

\begin{tabular}{|l|l|l|}
\hline $\begin{array}{l}\text { Interverwe9: } \\
\text { Tom Miner }\end{array}$ & $\begin{array}{l}\text { Intervew Date: } \\
\text { August 29, 2013 }\end{array}$ & $\begin{array}{l}\text { intervewer: } \\
\text { Unda Cottey }\end{array}$ \\
\hline
\end{tabular}

Current Role: Serf-employed consuttant subcontracted by UT-Batelle (UTB) and URSiCH2 Qak Ridge LLC (UCOR) for deactivation and decommissioning (DSD) project actuitles related to glove box and hot cell faclities located 3 , the Oak Fudge National Laboratory (ORNL).

Role and involvement in leotope Production at ORNL

1985 - 1992 (Operator)

$1992-2008$ (Operations Supervisor)

2008 - presert (Consultant)

Mr. Miner served as an operator and Operattons Supervisor for several faclitles, including Butong 3029. Mr. Miner retired in 2008 and subcontracts his servces to UTB and UCOR for continued support for hot cel and glove box faclitles located at the ORNL

Summary of Converaation

Mr. Miner recaled the following information related to ris experlence with Bulding 3029 :

Genera Bulding Comments

- The hot cels, especially cells 1,2, and 3, and the charging area were highly contaminated.

- Other areas of contamination included the glove bowes, drains, and vent pipes.

- All cobalt sources were removed from the Cobat Garden, except for two. Two were so badly degraded that they could not be removed.

- The paint on the celing and walls was coming on, under the paint there was contamination.

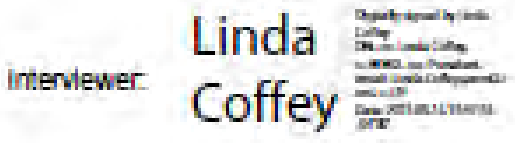




\section{ORAU/ORISE \\ Process Inventory Waste Project ORNL Building 3038}

\section{LIVING MEMORY SUMMARY}

September 2013

\section{Linda A. Coffey}

Waste Management Innovations, Inc.

305 S. Chamberlain Avenue

Rockwood, TN 37854

865-776-5041 


\section{INTRODUCTION}

The following information is derived from interviews of individuals that have personal knowledge pertaining to Bldg. 3038. This information was collected as part of a process to capture historical insights, usually long after the relevant events occurred. The opinions and comments from interview participants have not been verified, and these statements may contain erroneous or incomplete information.

Discussions were held with six individuals with experience in Bldg. 3038 ranging from 1962 through present day. Two were Target Group Technicians, promoted to Supervisors between 1962 and 1980; two were Chemical Operators, promoted to Operations Supervisors between 1975 and 1985; two supported the ORNL Hot Cells Project OR-22 during 2012, one as the Building Engineer and one as the Industrial Hygienist. Three of these individuals are currently employed at the Oak Ridge National Laboratory (ORNL), two continue to supply subcontract support to the ORNL, and one individual retired from the Isotopes Division in 1988.

\section{INTERVIEWS}

One former employee served as a Chemical Operator/Operations Supervisor before retiring from UT-Battelle in 2008 and continues to supply subcontract support for decontamination and decommissioning (D\&D) activities in Bldg. 3038. He stated that Bldg. 3038 was a research facility where many small-scale experiments and research from several divisions were conducted. Yttrium-90 and Strontium-90 work were conducted in the hot cells in the east end of the building. Plutonium sealed source welding was conducted in glove boxes in the west end of the building. These glove boxes went through D\&D in 2004 or 2005. Waste from this building was managed in accordance with ORNL procedures in place at the time. Individual waste items were double bagged and placed in stainless steel drums with another bag liner used to hold all waste within a drum. Strontium-90 and transuranic contaminated waste were segregated. The transuranic contaminated waste from the east and west ends of this building were segregated by operating area. Transuranic waste was not segregated by project or activity. The middle portion of the building was used for isotope shipping (including isotope sales) of radiological materials. The east end of the facility has been remediated. Glove boxes and hot cells on the east end and the yttrium cell were filled with foam in 2003. Bottles of liquid isotopes that were located in the Isotope Shipping Area, also known as the barricade area, have been removed. Ductwork located in the attic throughout the facility is deteriorating and suspect for contamination. The yttrium-90 duct that went to the filter house is deteriorating and causing yttrium and strontium contamination to fall into the operating area. The Alpha Handling Facility (AHF) hot cells are highly contaminated with plutonium, curium, americium, and other isotopes. The seven glove boxes in the AHF Annex are highly contaminated with numerous actinide isotopes, including plutonium and tritium. All were sprayed with fixative. Lots of releases occurred in the AHF area though the years. The facility has been decontaminated, however, contamination still exists in difficult to clean areas, (i.e., the shield tanks and under the hot cells are likely to be highly contaminated).

The other Chemical Operator/Operations Supervisor was involved primarily with the hot cells and glove box operations located on the west side of Bldg. 3038 in the AHF. Occasional radiological releases occurred when sources were transferred out of the hot cells. Contamination was usually confined to the area around the hot cells. However, contamination should be suspected underneath any painted surface, as painting over contaminated surfaces was a common decontamination technique. A major release occurred in the Shipping Area in the 1980s. The entire building was shut down for several months for

decontamination. The cause of the release was from a broken bottle of liquid that was stored in the barricade. As the liquid dried, it became airborne and contaminated the entire facility. 
The Isotope Research Materials Laboratory (IRML) is on the east side of Building 3038. While working with the IRML Group in the IRML Facility, one research technician/supervisor stated that he worked in glove boxes; supervised others that worked in glove boxes; worked with ceramics, metallurgy, high temperature metal and oxide evaporation processes; as well as ran the x-ray unit. Americium, plutonium, curium, and small amounts of californium were processed using reduction-distillation and oxide evaporation techniques. The Chemical Technology Division glove boxes were used primarily for plutonium oxide and plutonium carbide thermal dynamic studies. One glove box was used for promethium doped crystals, glasses and single crystals. On the north wall of the AHF were two glove boxes used for research and development $(R \& D)$. One $R \& D$ glove box contained a hot press where americium and curium pellets were processed for the fast breeder reactor program. Neptunium-237, uranium-233, and plutonium-239 were vaporized in an electron beam evaporator. Two of the hot cells were used to make plutonium-beryllium neutron sources. Contamination is likely to be everywhere in the IRML due to the number of releases of mostly actinides and some promethium. Some areas were not easily accessible for cleanup. Contamination should be expected when items are moved, as well as when the building is demolished. Three specific release events in the IRML were referenced during the discussion:

1. An americium release occurred in 1984 or 1985 which created contamination all over the east end of the building with count rates up to one million disintegrations per minute. All surfaces were contaminated. Duct work had to be removed. The room was cleaned up and put back into service. However, some areas were inaccessible for clean-up.

2. On the south side of the IRML a release occurred in front of the Low Geometry Counting Hood when a bottle of promethium-147 was shaken. The bottle cap had been ventilated, so as the bottle was shaken solution splattered all over the surrounding area.

3. Curium was released near the roll-mill glove box. The contamination cleanup included replacement of floor tiles.

The retired Target Group Technician worked in glove boxes and evaporators in both the IRML and the AHF, as well as in the hot cells in the AHF. He recalled that plutonium sources were welded in the welding box on the west end of the building. He also recalled that the numerous contamination events occurred during the time he worked there and referenced two specific events:

1. A spill occurred in the east end of the building when a source, encapsulated in plastic, was removed from a cell to show visitors touring the area. The plastic had a crack in it and contaminated the area, including the shoes of the visitors.

2. A release occurred in the AHF from a drain line that had a brass or copper fitting (instead of a stainless steel fitting) that wasn't welded. When an acid solution was poured down the hot drain, the drain leaked curium under the cell and onto the floor.

The Bldg. 3038 Engineer, who supported Hot Cells Project OR-22 as part of the U.S. Department of Energy (DOE) D\&D contractor team, conveyed information related to the condition of the building while he worked in the facility during 2012. The building was operated on temporary power provided by an auxiliary source because power panels had previously been removed from the building. The entire west end facility was smeared, external of the hot cells and glove boxes, for beryllium. The results for beryllium were negative within the facility. Legacy reports indicated that beryllium sources were used in the hot cells. Glove boxes and hoods throughout the facility were sprayed with fixative in preparation for 
removal, and some were removed. The AHF receiving area was used as a staging area for dress out and egress. Each of the five hot cells in the AHF has removable shielding that can be taken off the front of each hot cell. Removal of these hot cells will be difficult, as the shielding goes between the cells. There is a welded pass through between the cells. The manipulators in four of the hot cells were in very poor condition and unusable. The window of Cell 3 had been modified and made into a glove box type area with bag-in and bag-out ports. Cell 3 did not have any manipulators. The hot cells ventilation systems remained on-line. The inside of Hot Cells 2, 3, 4, and 5 were smeared but never analyzed. The glove boxes on the north side of the AHF were removed. The glove boxes in the AHF Annex were sprayed with fixative in preparation for removal. Containers, labeled as empty, were left inside the glove boxes. ORNL radiography confirmed that they were empty. Some of the glove boxes were approved for transfer to the TRU Waste Processing Center (TWPC) due to high transuranic activity content inside the glove boxes. Other glove boxes were to go out as low-level waste. The glove boxes remained on-line on the Bldg. 3038 ventilation system. Most of the bottles in the storage wells in the Isotope Shipping Area were removed. The lazy susan area remained highly contaminated with gamma activity. Lots of vials in the lazy susan area were packaged and removed. The tunnel is empty. All containers and the conveyer were removed. The IRML office and counting room were clean areas. The office was used as a dress area to prepare for entry into the facility. The remainder of the IRML was considered a contamination area. Hoods and glove boxes were foamed with fixative in preparation for removal. The Isotope Technology Process Area had several ceiling panels removed in order to access the ductwork to obtain radiological smears for characterization. Hoods in this area were foamed and removed.

The Industrial Hygienist stated that smears were collected from the ventilation systems throughout Bldg. 3038. These smears were analyzed for perchlorates and the results were negative. Four of the five hot cells were sampled before the Hot Cells Project OR-22 was shut down. The smears were stored in Cell 3. The smears collected from inside the hot cells in the AHF were not analyzed. 


\section{Record of Communication: Building 3038, Bobby Byrum}

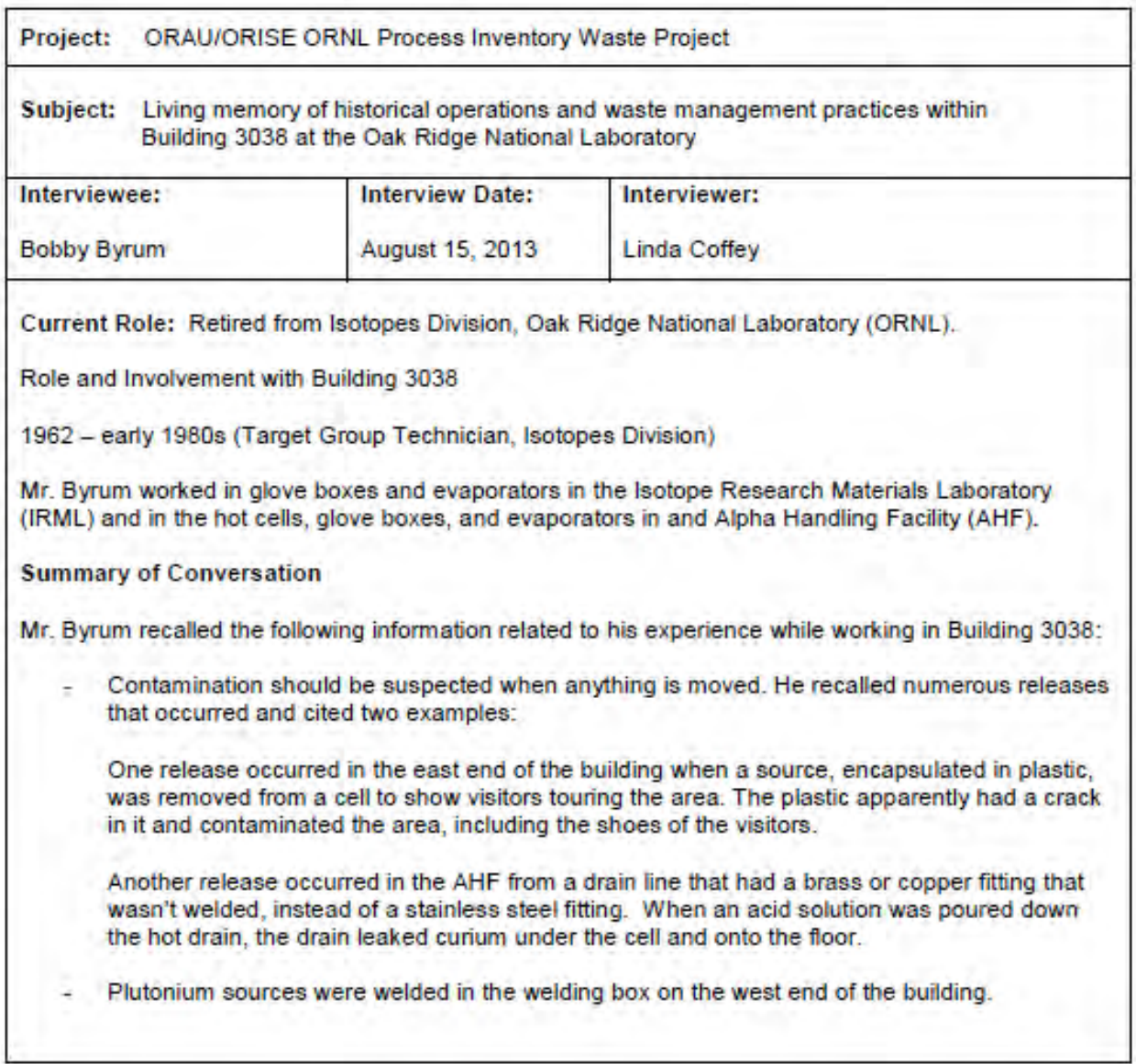

\begin{tabular}{|c|c|}
\hline Interviewer: & 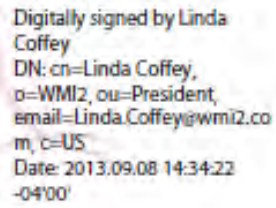 \\
\hline
\end{tabular}


Record of Communication: Building 3038, Tom Miner

\begin{tabular}{|l|l|l|}
\hline Project: & ORAU/ORISE ORNL Process Inventory Waste Project \\
\hline Subject: & $\begin{array}{l}\text { Living memory of historical operations and waste management practices within } \\
\text { Building } 3038 \text { at the Oak Ridge National Laboratory }\end{array}$ \\
\hline $\begin{array}{l}|l| \\
\text { Interviewee: }\end{array}$ & $\begin{array}{l}\text { Interview Date: } \\
\text { Tom Miner }\end{array}$ & $\begin{array}{l}\text { Anterviewer: } \\
\text { Linda Coffey }\end{array}$ \\
\hline
\end{tabular}

Current Role: Self-employed consultant subcontracted by UT-Battelle (UTB) and URS|CH2 Oak Ridge LLC (UCOR) for deactivation and decommissioning (D\&D) project activities related to glove box and hot cell facilities located at the Oak Ridge National Laboratory (ORNL).

Role and Involvement in Isotope Production at ORNL

1985 - 1992 (Operator)

1992 - 2008 (Operations Supervisor)

2008 - present (Consultant)

Mr. Miner served as an operator and Operations Supervisor for several facilities, including Building 3038. Mr. Miner retired in 2008 and subcontracts his services to UTB and UCOR for continued support for hot cell and glove box facilities located at the ORNL.

\section{Summary of Conversation}

Mr. Miner recalled the following information related to his experience while working in Building 3038 during 2012:

General Building Comments

- The east end of the facility has been remediated. All of the glove boxes and hot cells on the east end and the yttrium cell were filled with foam in 2003.

- All bottles of liquid isotopes that were located in the Isotope Shipping Area, also known as the barricade area, have been removed. There are plans for this area to be sprayed with fixative within the next two to three weeks.

- All ductwork located in the attic throughout the facility is deteriorating and suspect for contamination. The yttrium-90 duct that went to the filter house is deteriorating and causing yttrium and strontium contamination to fall into the operating area.

- The Alpha Handling Facility (AHF) hot cells are very contaminated with plutonium, curium, americium, and other isotopes. There are plans to spray these with fixative. Because they are so contaminated, smears will be taken after the fixative is applied, not before. The goal is to get the facility below a category 3 .

- The seven glove-boxes in the AHF annex are highly contaminated with numerous isotopes, including one with plutonium and another with tritium. Safety and Ecology Corporation (SEC) sprayed these with fixative last year however, smears were not taken at that time.

- Lots of releases occurred in the AHF area though the years. It's been decontaminated, however, contamination still exists in difficult to clean areas for example the shield tanks and under the hot cells is likely to be highly contaminated. 


\title{
Record of Communication: Building 3038, Tom Miner
}

\begin{abstract}
Mr. Miner was interviewed by Acceptable Knowledge Expert Tom Krause on May 18, 2005 related to "Historical operations and waste management at Building 3038." The following report represents Mr. Miner's responses during that interview (Tracking Document C097, TRU/Alpha Low Level Waste Treatment Project, TRU-OPS-1110, Rev. B.).
\end{abstract}

^Note: Mr. Miner has been involved with Buildings 3028, 3038, 3517, 3026D, 3029, 3505, 4507,3042 (ORR), and 3010 (BSR).

What is your connection with Building 3038 ? -Or-What was your position at Building 3038 ? Mr. Miner was an operator from 1985 to 1992 and has been the Operations Supervisor from 1992 to present.

During what time frame were you involved with operations at Building 3038 ?

1985 to present.

What programs or projects were conducted in this building?

This was a research facility and many small-scale experiments and research from many divisions was conducted here. Y-90/Sr-90 work was conducted in the hot cells in the East end of the building.

$P u$ sealed source welding was conducted in glove boxes in the West end of the building. These glove boxes were $D \& D^{\prime} d$ in 2004.2005 . The middle portion of the building was used for rad materials storage and shipping (including isotope sales).

What is your recollection of waste management practices at this building?

Waste from this building was managed in accordance with ORNL wide procedures in place at the time.

Are you familiar with how waste from this building was packaged? If yes, can you provide details (e.g., layers of confinement, filters on drum or inner packages, rigid drums liners)?

Individual items were double-bagged and placed in stainless steel drums with another bag liner used to hold all waste within a drum.

Was waste segregated at this building? If yes, how?

Sr-90 waste and PU-contaminated waste was segregated. TRU-contaminated waste from the east and west ends of this building were segregated by area, only due to convenience. TRU waste was not segregated by project or activity.

Is there anyone else that may be able to help with this information? No one else still available."

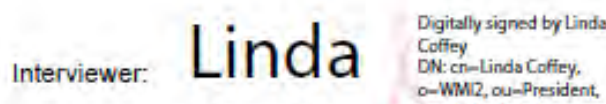

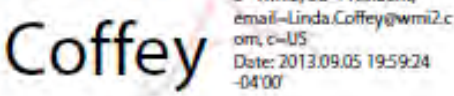


Record of Communication: Building 3038, David Drake

\begin{tabular}{|l|l|l|}
\hline Project: & ORAU/ORISE ORNL Process Inventory Waste Project \\
\hline Subject: & $\begin{array}{l}\text { Living memory of historical operations and waste management practices within } \\
\text { Building } 3038 \text { at the Oak Ridge National Laboratory }\end{array}$ \\
\hline $\begin{array}{l}\text { Interviewee: } \\
\text { David Drake }\end{array}$ & $\begin{array}{l}\text { Interview Date: } \\
\text { August 15, 2013 }\end{array}$ & $\begin{array}{l}\text { Interviewer: } \\
\text { Linda Coffey }\end{array}$ \\
\hline
\end{tabular}

Current Role: Group Leader for Nuclear Research Support and Nuclear Science and Engineering Directorate at UT-Battelle (UTB) located at the Oak Ridge National Laboratory (ORNL).

Role and Involvement with Building 3038

1975 - 1985 (Chemical Operator)

1985 - 1991 (Operations Supervisor)

Mr. Drake while serving as a chemical operator and as an operations supervisor between 1975 and 1991, Mr. Drake worked in several, including Building 3038.

Summary of Conversation

Mr. Drake recalled the following information related to his experience while working in Building 3038 :

- Mr. Drake's involvement with Building 3038 was in the west side of the building, in the Alpha Handling Facility (AHF) with the hot cells and the glove box operations.

- From time to time releases occurred when sources were transferred out of the hot cells. Normally contamination was confined to the area where the work was being done.

- Mr. Drake stated that contamination should be suspected underneath any painted surface, as that was a common technique for cleanup.

- Mr. Drake recalls a major release in the Shipping Area in the late $1980 \mathrm{~s}$. The entire building was shut down for several months for decontamination clean up. He recalled that the cause of the release was from a broken bottle of liquid that was stored in the barricade. As the liquid dried, it became airborne and contaminated the entire facility.

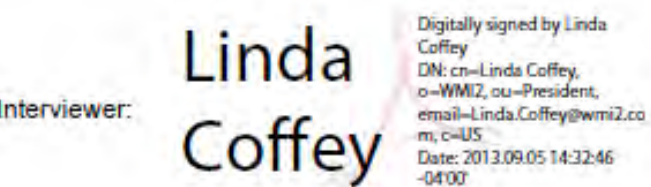


Record of Communication: Building 3038, Marwan Bader

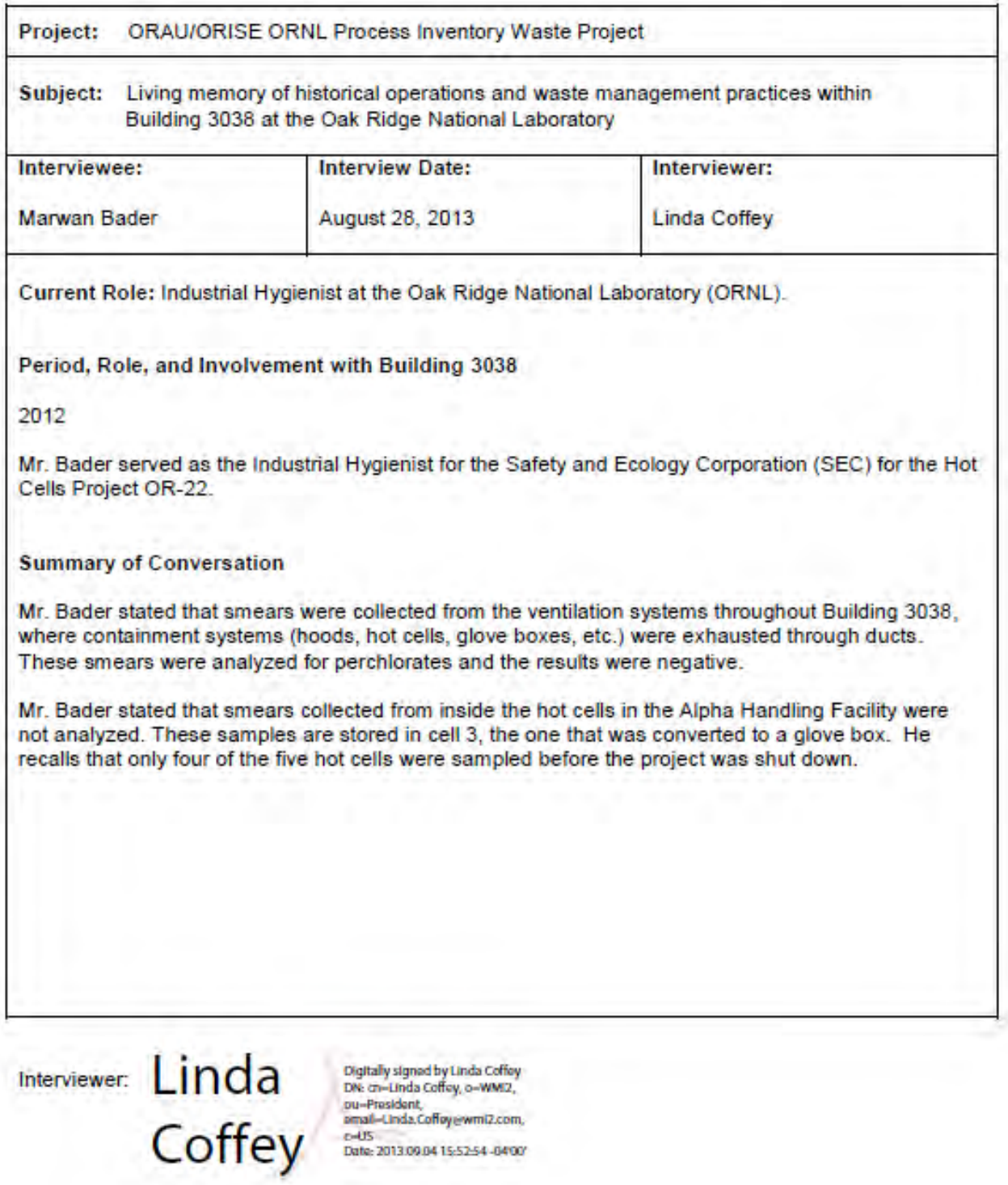


Record of Communication: Building 3038 , Kenton Wilson

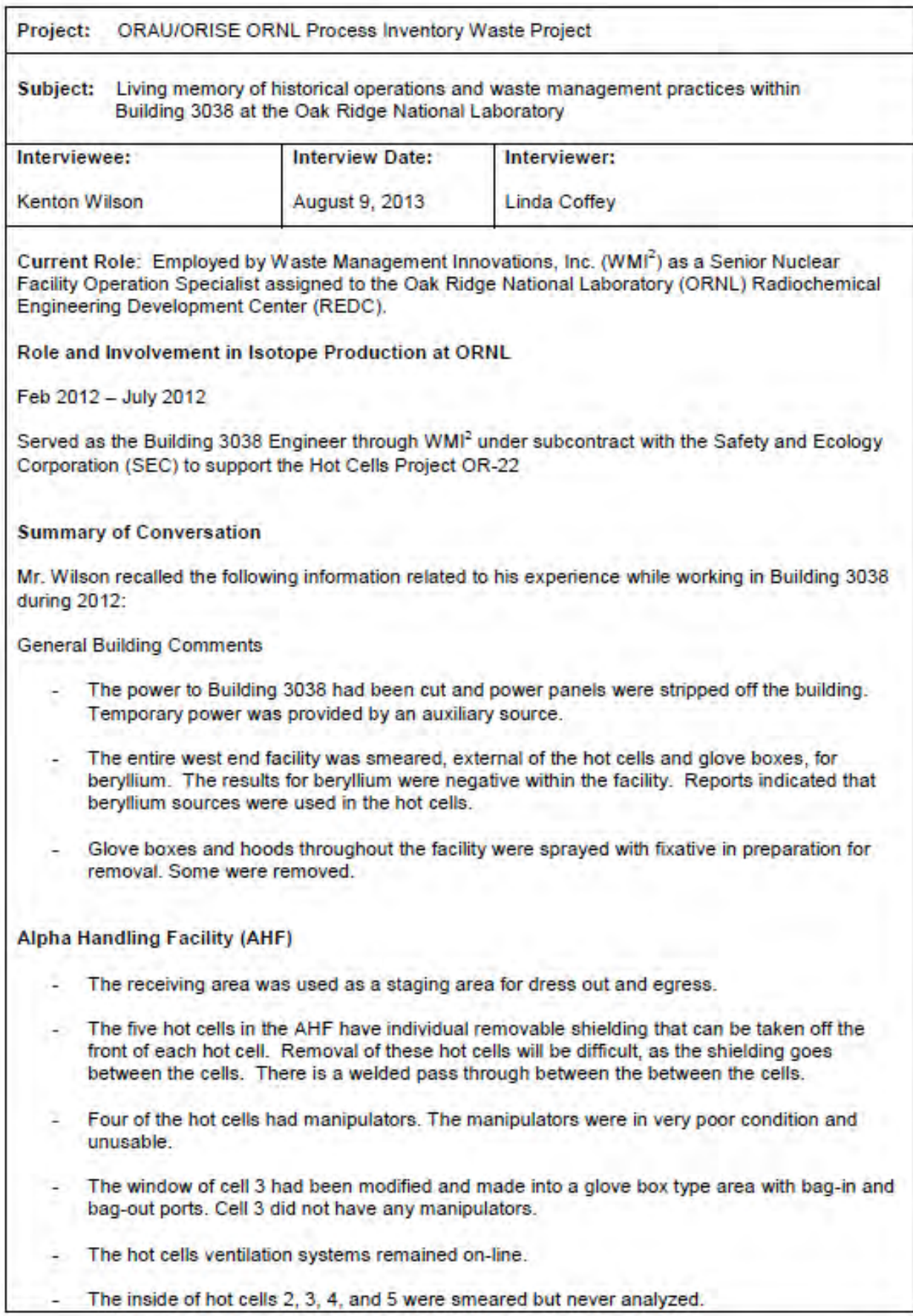

Page 1 of 2 
Record of Communication: Building 3038, Scott Aaron

\begin{tabular}{|l|l|l|}
\hline Project: ORAU/ORISE ORNL Process Inventory Waste Project \\
\hline $\begin{array}{l}\text { Subject: } \\
\text { Living memory of historical operations and waste management practices within } \\
\text { Building } 3038 \text { at the Oak Ridge National Laboratory }\end{array}$ \\
\begin{tabular}{l|l|l|} 
Interviewee: & Interview Date: & Interviewer: \\
W. Scott Aaron & August 19,2013 & Linda Coffey \\
\hline
\end{tabular}
\end{tabular}

Current Role: Isotope Development Group, Nuclear Science \& Technology Division at the Oak Ridge National Laboratory (ORNL)

Period, Role, and Involvement with Building 3038

$1976-1990$

Mr. Aaron began working in Building 3038 as a technician with the Isotope Research Materials Laboratory (IRML) group. While working in the facility, he worked in glove boxes; supervised others that worked in glove boxes; worked with ceramics, metallurgy, high temperature metal and oxide evaporation processes; he also ran the $x$-ray unit; as well as assisted with development of the Safety Analysis Reports (SARS) for Building 3038.

\section{Summary of Conversation}

Mr. Aaron recalled the following details related to the IMRL areas on the east end of Building 3038:

- Americium, plutonium, curium, and small amounts of californium were processed using reduction-distillation and oxide evaporation techniques.

- The Chemical Technology glove boxes were used primarily for plutonium oxide and plutonium carbide thermal dynamic studies.

- One glove box was used for promethium doped crystals, glasses and single crystals.

Mr. Aaron recalled the following details related to the Alpha Handling Facility in Building 3038 :

- On north wall there were two glove boxes that contained evaporators and a glove box were used for research and development (R\&D). Neptunium-237, uranium-233, and plutonium-239 were vaporized in an electron beam evaporator.

- The R\&D glove box contained a hot press where americium and curium pellets were processed for the fast breeder reactor program.

- Two of the hot cells were used to make plutonium beryllium neutron sources.

When asked about areas likely to have contamination today, Mr. Aaron stated that contamination is likely to be everywhere in the IMRL as there was a lot of potential for release of almost all the actinides and some promethium as well. Some areas were not easily accessible for cleanup. Contamination should be expected when items are moved, as well as, when the building demolished. He recalled three specific release incidents in the IMRL area: 


\section{Record of Communication: Building 3038, Scott Aaron}

1. An americium release occurred in 1984 or 1985 which created contamination all over the east end of the building, up to one million DPM. All surfaces were contaminated. Duct work had to be removed. The room was cleaned up and put back into service. However, some areas were inaccessible for clean-up.

2. On the south side of the IRML a second release occurred in front of the Low Geometry Counting Hood when a bottle of promethium-147 was shaken. The bottle cap was ventilated, so as the bottle was shaken, solution splattered all over the surrounding area.

3. Curium was released near the roll-mill glove box. The contamination cleanup included replacement of floor tiles.

While Mr. Aaron did not work in the west end of Building 3038, he recalled activities that occurred in the Isotope Technology Process Area:

- Stronium-90 and yttrium-90 were processed in a hot cell for medical applications.

- Tritium may have been used in this area to support the tritium light program. Glass tubes were containing fluorescent powder were filled with tritium to produce radio-luminescent lights.

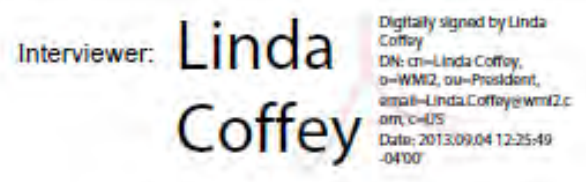




\section{ORAU/ORISE \\ Process Inventory Waste Project \\ ORNL Building 3042}

\section{LIVING MEMORY SUMMARY}

September 2013

\section{Linda A. Coffey}

Waste Management Innovations, Inc.

305 S. Chamberlain Avenue

Rockwood, TN 37854

865-776-5041 


\section{INTRODUCTION}

The following information is derived from interviews of individuals that have personal knowledge pertaining to the Oak Ridge Research Reactor, Bldg. 3042. This information was collected as part of a process to capture historical insights, usually long after the relevant events occurred. The opinions and comments from interview participants have not been verified, and these statements may contain erroneous or incomplete information.

Discussions were held with two individuals with experience ranging from 1983 to 2013 in Bldg. 3042. Both individuals served as the Facility Manager; one also served as the Assistant Facility Manager while the facility was operational. Both individuals continue to provide support to ORNL facilities.

\section{INTERVIEWS}

One individual was the Assistant Facility Manager from 1983-1986, then Facility Manager from 19871997. He stated that the piping had numerous leaks due to the original reactor design where aluminum piping was used. The line was removed and the loop was shortened. All that was left was an abandoned primary piping line out in the field that went toward a heat exchanger and a cooling tower that are no longer in existence.

The current Facility Manager has served in this capacity since 2008. He stated the facility is in standby, waiting for D\&D. The building is in pretty good condition. Operational systems include a wet pipe sprinkler system, steam for heat, electricity, plant air, potable water, a radiation alarm system, and the reactor pool circulation system. There is legacy material in the reactor pool. Demineralized water supplied to the reactor pool helps to minimize corrosion of the pool liner. The inactive filter pits for the cell vent system have been isolated. 
Record of Communication: Building 3042, Bruce Howard

Project ORAuONSE ORN Process inventory Wate Projed

Subject: Living menory of historical operatons and waste mangoment practces wtthin Bulding 3042 at the Oak Ridge Natlonal Laboratory

\begin{tabular}{|l|l|l}
\hline Intervewos: & Intervaw Date: & Intarvawer: \\
Eruce H. Howard & Septenber 19, 2013 & Unda Cotley \\
\hline
\end{tabular}

Role and invovement with Bullding 3042

2008 - presert (frad iy Manzog)

As Facd ty Marager, Mr. Howard's Inohement with Eulding 3042 hcudes monthly hopectlons to ensure that the facilty ls secure, checks tor changing tacilty condtons, emegency lighting, lakage, and that vegetation is controled around the fac ly.

Summary of Converaation

- The tadity is in standby awailing decomnissioning and decontamination (DSDL.

- Faclity is unocapled.

- Operatonai systems indude a wet pjpe sornkjer system, steam for heat, electity, pant ari: and ootable water.

- The reador poo draiston syetem ls cperatonal

- There is legacy matetal in the rescor pool.

- Demheralzed water suppled to he reactor pool helps to mininge corrobn of the pool liner.

- The buiding is in prety good conditon, no signiflbart leaks.

- The fadity has an coeratonal radaton alam system

- The inache firer pls tor the cel vent system have been isolated.

intevewer Linda A.

Coffey 
Record of Communication: Building 3042, Mark Ford

Project: ORALIOFISE ORNL. Process inventory waste Project

Subject: LJving menory of historica operations and waste managenent practices within Bulding 3042 at the Oak Ridge National Laboratory

\begin{tabular}{|l|l|l}
\hline Interviewe9: & $\begin{array}{l}\text { Intervew Date: } \\
\text { Mark Ford }\end{array}$ & $\begin{array}{l}\text { September 11, 2013 } \\
\text { Sewda Cotley }\end{array}$ \\
\hline
\end{tabular}

Role and involvement with Bullding 3042

1963 - 1986 (Assistant Faclity Manager)

1987 - 1997 (Faclity Manager

As Assistant Faclity Manzger, Mr. Ford was responslble for reports and experiments conducted in the faclity. As the Facility Manager, Mr. Ford w3s responsible for survellance and maintenance

activities.

Summary of Conversation

- The piping had numerous leaks due to the original reactor design where alumirum piping was used. The ine was removed and the locp was shortened. As that was left was an abandoned primary piping ine out in the feld that went toward a heat exchanger and a cocling tower that are no longer hin existence.

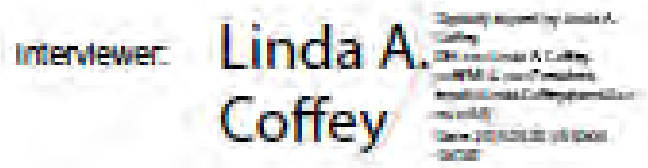




\title{
ORAU/ORISE \\ Process Inventory Waste Project ORNL Building 3517
}

\section{LIVING MEMORY SUMMARY}

September 2013

\author{
Linda A. Coffey
}

Waste Management Innovations, Inc.

305 S. Chamberlain Avenue

Rockwood, TN 37854

865-776-5041 


\section{INTRODUCTION}

The following information is derived from interviews of individuals that have personal knowledge pertaining to the Fission Product Development Laboratory, Bldg. 3517. This information was collected as part of a process to capture historical insights, usually long after the relevant events occurred. The opinions and comments from interview participants have not been verified, and these statements may contain erroneous or incomplete information.

Discussions were held with five individuals with experience in Bldg.3517 ranging from 1959 through present day. One was a Chemical Operator; two were Chemical Operators, promoted to Operations Supervisors; one was a Program Manager for the Isotope Facility Shutdown and currently serves as the Facility Manager; and one was the Group Leader in the Isotopes Operations Division. Three individuals continue to supply support to ORNL, and two individuals retired from ORNL.

\section{INTERVIEWS}

One retiree served in Bldg.3517 as a Chemical Operator from 1959 to 1962 . He stated that Bldg.3517 was a production facility that was highly contaminated with lead, strontium-90, and cesium-137. Cell 11 had a storage well with multiple sources stored in it. Cell 13 had holes in the stainless steel bottom and leaked. Cell 14 had a lot of lead stored in it and will be difficult to remove since the window views are obstructed. Hydrochloric acid was often used for decontamination and cleanup. Contamination should be expected when items are moved. He was told that the dirt under Bldg.3517 was contaminated.

One current ORNL employee served as a Chemical Operator from 1979 to 1985. He worked in several facilities during that time, including Building 3517. He was Operations Supervisor for several facilities until 1991. He did not have direct responsibility for Bldg.3517; however, he filled in as Operations Supervisor there, as needed. He stated that the chemical cell bank valves were highly contaminated; one had a dose rate up to $300 \mathrm{R} / \mathrm{hr}$. The manipulator cells had multiple contaminates primarily, strontium-90 and cesium-137. Manipulators were pulled weekly, and sometimes daily, there was always contamination. The facility produced numerous isotopes. All floors, including the lunch room, were contaminated. Contamination was found in the ventilation ductwork in the late 1980s or early 1990s. Everything in the entire building was suspect for radioactive contamination. He heard that the high bay area was cleaned up and no longer considered a contamination zone.

One retiree worked in several of the Isotopes Facilities during his forty-one year career as the Group Leader in the Isotopes Department, Operations Division. He was also responsible for the D\&D of fourteen facilities, as well as the lead author of the ORNL Final D\&D Report for Bldg.3517. He retired in 1988. He stated that this facility was used for separating fission products, primarily strontium-90 and cesium-137, as well as cerium-144, promethium-147, and technetium-99. Cesium-137 was used in numerous applications. Strontium-90 was used as power sources for electric generators. Smear results, radiation levels, operations history, drawings, and drawing lists can be found in the final Bldg.3517 D\&D report.

Before retiring in 2008, one individual served as a Chemical Operator from 1985 to 1992, then as an Operations Supervisor. He currently supplies subcontract support for hot cell and glove box facilities located at the ORNL. He stated that more sources were fabricated in this facility than any other isotope facility. Cesium and strontium were the major elements of concern in this facility. Releases occurred all over the facility. Areas covered by paint, areas above seven to eight feet, and areas under floor tiles should be suspect for contamination. This facility has as much inventory as it did in 1975. The RTGs that 
were stored outside near the northeast corner of the building are gone, except for the BUP-500. The storage well in Cell 10 west currently has sealed sources stored in it that have not been viewed in at least five years; the cell window is cloudy. The chiller unit that kept the storage well cool has been out of service for several years, so no one knows the condition of these sources. There are many other hazards in this facility and they are identified in The Documented Safety Analysis for Building 3517, DSA-OR-35170013,, Rev. 12, U.S. Department of Energy Office of Environmental Management, Oak Ridge, TN.

One individual served as the ORNL Program Manager from 1991 to 2004 and currently serves as the Facility Manager. He stated systems are on stand-by. The primary concern is the deteriorating utility infrastructure, old piping, steam pipes, water pipes, air lines, etc., as they age. The facility has poor lighting. Utilities are shut off to the cells. Cell 11 has a laboratory beaker with sources in it. Cell 10 west has a water-filled storage well with sources down in it. The cell window is clouded up and cannot be seen through. The underground cells behind the building contain contaminated waste tanks. The underground ductwork on the east side that ventilates the building is contaminated. Directly behind the building are two waste tanks, W19 and W20, and the soil around them is contaminated. Sometimes the contamination washes off to the north tank pad and causes it to be contaminated. 
Project: ORAuIORISE ORNL Process inventory Waste Project

Subject: Living memory of ivstorical operations and waste management practices within Bulding 3517 at the Oak Ridge National Laboratory

\begin{tabular}{|c|c|c|}
\hline Thizervewe9: & Intervaw Date: & Tinterviewer: \\
\hline Kart Hat' & September 3,2013 & Unda Cottey \\
\hline
\end{tabular}

Current Riole: Retired, ORNL isotopes Division

Role and involvernent with Bullding 3517

1980 - 1968 (Isotopes Operation Dvision, Group Leader)

Mr. Hart worked in several of the ORNL faclities durng his 41 year career. He became involved with Eulding 3517 in the late 1960's. He was responsible for decormmissioning and decortamination (DSD) for fourteen faci ties, Inciuding Bulding 3517.

Summary of Conversation

Mr. Haif stated the following information Bullding 3517 :

- This was a facility used for separating fission products, primarlly strontum-90 and ceslum137 , as well as centum-144, promethium-147, and fechnedum-99. Cesium-137 was used in numerous appications. Strontum-90 was used as power sources for electric generators. He was involved in some of the work on the power generators.

- Mr. Hatl was the lead author of the DSD reports for Bulding 3517. Smear result, radation levels, operatiors history, drawings, and crawing lists can be found in the Final Bulliding 3517 DSD report.

Intervewer.

Linda A. Coffey

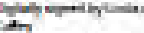
sinimatis 


\section{Record of Communication: Building 3517, David Drake}

Project: ORAuIORISE ORNL Process inventory Waste Project

Subject: Living memory of historical operations and waste management practices within Bulding 3517 at the Oak Ridge National Laboratory

\begin{tabular}{|l|l|l|}
\hline Intarvewe9: & Intervaw Date: & Interviewer: \\
David Drake & August 15,2013 & Unda Cottey
\end{tabular}

Current Fiole: Group Leader for Nuclear Research Support and Nuclear Sclence and Engineering Directorate at UT-Exatele (UTB) located at the Oak Ridge National Laboratory (OFNL).

Role and Involvement with Bullding 3517

$1979-1985$ (Chemicar Cperator -2 years were in Euliding 3517 )

1985 - 1991 (Operations Supervisor)

Mr. Drake was a chemical operator in Buliding 3517 for two years. Bulding 3517 was not one of the facilites that he was responslole for as an Operations Supervisor, however he flled in 36 supervisor

as needed.

Summary of Conversation

Mr. Drake recaled the folowing information related to his experience whle working in Bulding 3517 :

- Av the cell barks were highly contaminated. The manipuator cels had multiple contaminates, primarily, strontum-90 and cesium- 137 .

- He heard that the high bay area was cleaned up and not considered a " $C$ " zone anymore.

- The chemical cell banks, which dd not have manipuators, had a lot of very hot valves. One cel they were tying to clean out w36 $300 \mathrm{R} / \mathrm{hr}$, and they couldn't deal with it that hot.

- The faclity produced numercus isotopes.

- Manipulators were puled weekly and sometmes daly. There was always contamination.

- Av the foors, even the lunch room were contaminated from contaminaton that was tracked in.

- Contamination was found in the HVAC ductwork in the late 1960 s to eariy 19906.

- Everything in the entlire buliding was suspect for radoactive contamination.

Intervewer. Linda A.
Coffey 


\section{Record of Communication: Building 3517, Bobby Byrum}

\begin{tabular}{|c|c|c|c|}
\hline subject: & \multicolumn{3}{|c|}{$\begin{array}{l}\text { Living memory of historical operations and waste management practices within } \\
\text { Bulloing } 3517 \text { at the Oak Ridge National Laboratory }\end{array}$} \\
\hline Tntervewe & & InterWew Date: & Tntervewer: \\
\hline Bobby Eyr & rum & August 15,2013 & Unda Cortey \\
\hline
\end{tabular}

Current Fiole: Retired from isotopes Division, Qak Ridge National Laboratory (ORNL).

Role and involvement with Bullding 3517

1959 - 1962 (Chemical Operator)

summary of Conversation

Mr. Byrum recalled the following informaton reizted to Ns experlence whlle wotking in Buiding 3517 :

- Bullding 3517 was a production facilty and was highly contaminated wth lead, stronttum-90, and cesium-137. Cortamination should be expected when items are moved.

- Hydrochiortc acid was ofen used for decontamination and cleanup.

- Cel 11 had a storage well wth multple sources stored in it.

- Cell 13 had holes in the stainless steel bottom and leaks.

- Cell 14 had a lot of lead stored in it, which wil be diffcut to remove since you cannot see through the windows.

- He was toid that the dirt under Bulding 3517 is contaminated from it.

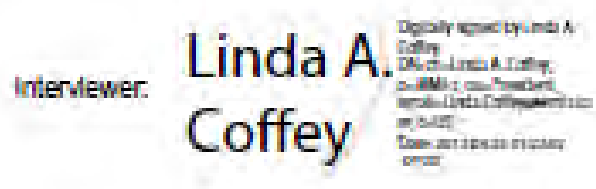


Project. ORAUIOFISE ORN Process Inventory Waste Project.

Subject: Living memory of historica coerations and waste management practioes within Bulding 3517 at the Oak Rldge National Laboratory

Tnterverwe9:

Tom Miner

\begin{tabular}{|l|l}
\hline Intervew Date: & Interviewer: \\
August 29,2013 & unda Cottey
\end{tabular}

Current Role: Ser-employed consultant subcontracted by UT-Battelle (UTB) and URSICH2 Oak Ridge LLC (UCOR) for deactivation and decommissioning (DSD) project actilties reiated to glove bor and hot cel faclities located at the Oak Fudge Natoral Laboratory (CRNL).

Role and involvement with Bullding 3517

1985 - 1992 (Operator)

$1992-2009$ (Coerations Supervison)

2008 - presert (Consultant)

Mr. Miner served as an operator and Operations Supervisor for several faciltes, Including Bulding 3517. Mr. Miner retired in 2008 and subcontracts his services to UTB and UCOR for continued support for hot cell and glove box faclitles located at the CRNL

Summary of Conversation

Mr. Miner recaled the following information related to his experlence with Euiding 3517:

- Ceslum and strontum are the major isotopic concerrs in this facility.

- Releases occurred all over the faclity. Aveas covered by paint, areas above seven to elght feet, and areas under toor tles most ikey are cortaminated.

- More scurces were fabricated in this faclity than any other isolope taclity.

- This tacllity has as much inventory $x$ It dd in 1975 . Nothing has been unioaded out of this faclity. It's not realy a survellance and mantenance faclity, it contains material.

- The Radiolsotope Thermoelectric Generators (RT GS) that were stored outside by the northeast comer of the buliding are gone, except for the BUP. 500 .

- The storage well in cell 10 west has seared sources stored in it that have not been vieved in at least tive years. The cel window is doudy and you cannot see inslde. The chller unit that kept the storage well cool has been out of senvice for several years, 50 no one knows the condtion of these sources.

- There are many other hazards with this faclity and they are identilned in The Documented Sarety Arialysis for Eullohng 3517, DSA-CR-3517-0013, Rev. 8.

intervewer:

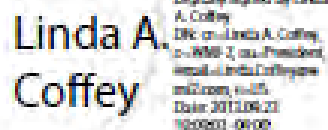

Poge 1 of 1 
Record of Communication: Building 3517, Robert Eversole

\begin{tabular}{|c|c|c|c|}
\hline Project: & \multicolumn{3}{|c|}{ ORALUORISE ORN Process Inventory Waste Project } \\
\hline Subject: & \multicolumn{3}{|c|}{$\begin{array}{l}\text { Living memory of historical operations and waste management practices within } \\
\text { Bulding } 3517 \text { at the Ozk Ridge National Laboratory }\end{array}$} \\
\hline $\begin{array}{l}\text { Tntarvewe } \\
\text { Robert ( } \mathrm{Bc}\end{array}$ & D): Eversole & $\begin{array}{l}\text { Intervew Date: } \\
\text { September } 18,2013\end{array}$ & $\begin{array}{l}\text { Tnterviewer: } \\
\text { Unda Cottey }\end{array}$ \\
\hline
\end{tabular}

Role and Inwolvement with Bullding 3517

1991 - 2004 (Program Manager)

2004 - present (Facilty Nanager)

Summary of Conversation

Mr. Eversole recalied the following information related to his experlence with Bus ding 3517 :

- Primary concern is the deteriorating urility intrastuchure, old piping, steam pipes, water plpes, air lines, etc., as they age.

- The faclity has poor lighting. There is a maintenance request to replace a lot of the light foctures and balasts.

- Cell 11 has a laborztory becker whth sources in it.

- Cell 10 west has a water-flled storage well wrin sources down in it. The cell window is couded up and cannot be seen through

- Uoirtes are shut or to the cells.

- The underground cels behind the bullding cortain waste tanks that are contaminated

- The ductwork that ventlates the bullding is underground on the east side is contaminated.

- Directy behind 3517 are two waste tanks, W19 and W20. The soll around them is contaminated. Sometimes the contamination washes of to the north tank pad and causes it be a contaminated area.

- Systems are on stand-by.

Imtervewer. Linda A
Coffey 


\section{ORAU/ORISE \\ Process Inventory Waste Project \\ ORNL Building 4507}

\section{LIVING MEMORY SUMMARY}

September 2013

\section{Linda A. Coffey}

Waste Management Innovations, Inc.

305 S. Chamberlain Avenue

Rockwood, TN 37854

865-776-5041 


\section{INTRODUCTION}

The following information is derived from interviews of individuals that have personal knowledge pertaining to the High Radiation Level Chemical Development Laboratory in Bldg. 4507. This information was collected as part of a process to capture historical insights, usually long after the relevant events occurred. The opinions and comments from interview participants have not been verified, and these statements may contain erroneous or incomplete information.

Discussions were held with two individuals with experience in Bldg.4507 ranging from 1963 through present day. One was a Hot Cell Operator in 1963 and 1964 and one was an Operations Supervisor from 2003 through 2005. One individual is currently employed with Waste Management Innovations, Inc. $\left(\mathrm{WMI}^{2}\right)$ as a Senior Nuclear Facility Operation Specialist currently assigned to the Oak Ridge National Laboratory (ORNL) Radiochemical Engineering Development Center (REDC), Bldg.7920; and the other individual retired from ORNL in 2008 and continues to supply subcontract support to the ORNL for hot cell and glove box facilities.

\section{INTERVIEWS}

The Hot Cell Operator developed chemical processing steps, procedures, and process flowsheets that were later used at the Radiochemical Engineering Development Center, Bldg.7920. The processes conducted in cells 3 and 4 were curium recovery processes. Cells 3 and 4 were used for preliminary operations for the 7920 facility. There were two campaigns to produce curium-242 for the Oak Ridge Research Reactor. Curium received from Savannah River was stored in an underground tank outside Bldg.4507. The solution was piped into Cell 4 so material could be drawn from outside for processing. In the late 1960s, the processing operation was moved to the newly constructed REDC facility. After the move, curium continued to be drawn into cell 4 in preparation for relocation of the material to the REDC operations. During the 1990s, the underground processing tank was removed and the processing lines were cut. The decommissioning crew assumed the tank was empty and never used. The tank was empty and had been flushed, however it was not free from activity and resulted in a contamination release to the immediate work area and uptakes to a couple of the workers.

The former Operations Supervisor was involved in decontamination activities in recent years. The only place in the facility he found contamination was in the hot cells. The charging areas to the hot cells were fairly clean. Fixative was applied to the hot cells and the hot cell ventilation system going to the 3039 Stack was shut off. A small local unit was installed in its place. Smears were taken before the hot cells were fogged with fixative and he has the data. There was contamination in the filter pit ductwork, so at some point in time there had been a lot of contamination released inside the building. The filter was removed from the filter pit and the pit was grouted in 2013. The small glove box on the second level of the mezzanine had lead in it. It was foamed with fixative and disposed of. The small cell above the hot cell bank in the penthouse, was also sprayed with fixative and disposed of. When he began working on decontamination activities, he tried to locate someone with legacy process knowledge and was unable to locate anyone that actually operated the facility. 


\section{Record of Communication: Building 4507, Tom Miner}

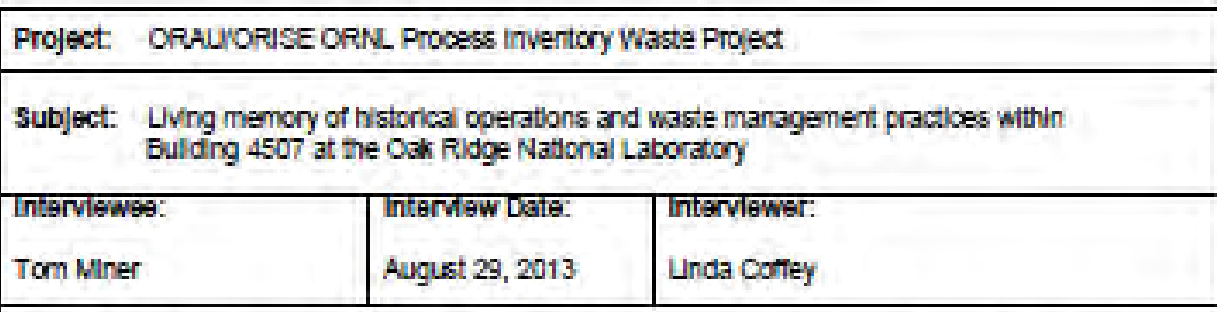

Current fole: Seliemoloyed consultant subcontracted by UT-Bathele (UTB) and URS|CH2 Oak Ridge LLC (UCOR) for deactivation and decommissioning (DSD) project actulties related to glove box and hot cell faclitles located at the Oak Fidge National Laboratory (ORNL)

Role and invoivement with Euliding 4507.

2003 - 2005 (Operations Supervisor)

2008 - presert (Consultant)

Mr. Miner served as a Chemical Operator and Operatons Supervisor for severa hot cell and giove box facilties located at the OFNL induding Building 4507, Mr. Miner conducted DSD activiles in Eulding 4507 .

Summary of Conversarion

Mr. Miner recaled the following information related to ris experlence uhlle working in Eulding 4507:

- The ony place in the facility he found contamination was in the hot cels. The charging areas to the hot cels were pretty clean, 36 far as contamination goes.

- Fxattve was appled to the hot cels, so the hot cel ventiation system going to the 3039 Stack could be shut of. A small local unit was installed in Its place. Smears were taken before the hot cels were fogged woth forative. Mr. Miner has the data.

- The fiter was removed fton the filter pt, and the pit was grouted in 2013. There was contamination in the ilter pit ductuork, so at. some point in time there had been a lot of contamination released inside 4507.

- The smal glove box on the second level of the mezzanine had lead in it. It was foamed with fixative, removed, and disposed of.

- The small cel above the hot cel bark in the penthouse, was also sprayed win flxative and disposed $\alpha$.

- Mr. Miner trled to locate someone with some legacy process knowledge and was unable to locate anyone that actualy operated the facility.

$\begin{aligned} & \text { intervewer. Linda } \text { (offey } \\ & \text { Cof }\end{aligned}$ 


\section{Record of Communication: Building 4507, Fred Chattin}

Project: ORAUNORISE ORNL Process inventory waste Project.

Subject: Living memory of listorical operations and waste management practices within Bulding 4507 at the Oak Ridge National Laboratory

\begin{tabular}{|c|c|c|}
\hline Thtarvewอ9: & Intervaw Date: & Tinterviewer: \\
\hline Fred Chattin. & September $4,2 \mathrm{D} 13$ & Unda Cottey \\
\hline
\end{tabular}

Current Fole: Emproyed by Waste Management Innovatons, inc. (WMII ${ }^{2}$ ) as a Serior Nuclear Facility Operaton Specialist assigned to the Oak Rudge National Laboratory (OFNL) Radlochemica: Englineering Development Center (REDC)

Role and Invoivement win Bullaing 4507

1963 - 1964 (Hot Cell Operator)

As a Hot Cell Operator working in Bullding 4507, Mr. Chatth, developed chemical processing step6, procedures, and process fowsheets that were later used at the Radlochemical Engineering

Development Center in Bulding 7920 .

Summary of Conversation

Mr. Chatun recalled the folowing infomation related to his expenence whlle working in Bulding 4507:

- The processes conducted in cells 3 and 4 were a curtum recovery processes. Cells 3 and 4 were used for prellminary operatlons for the 7920 facilin. Curtum recelved from Savannah Fiver was stored in an underground tark outside 4507 . The solution was piped into cell 450 material could be drawn from outside for processing. Even atter the process was moved into the 7920 facilty in the late $1960 \mathrm{~d}$, the curium contrued to be drawn into cell 4 in preparation for relocation of the material to the REDC.

- During the 19906, the underground processing tark w3s removed and processing lines were cut. The decommlssioning crew assumed the tark was empty and never used. The tank was empty and fushed of processing solution, however it was not free from activity and resuted in a coniamination reiease to the inmedlate work area and uptakes to a couple of the workers.

- There were two campaigs to produce curtum-242 for the Oak Roge Research Reactor. intervewer: Linda

Coffey 


\section{ORAU/ORISE \\ Process Inventory Waste Project ORNL Building 7500}

\section{LIVING MEMORY SUMMARY}

September 2013

\section{Linda A. Coffey}

Waste Management Innovations, Inc.

305 S. Chamberlain Avenue

Rockwood, TN 37854

865-776-5041 


\section{INTRODUCTION}

The following information is derived from interviews of individuals that have personal knowledge pertaining to the Homogeneous Reactor Experiment Facility, Bldg. 7500. This information was collected as part of a process to capture historical insights, usually long after the relevant events occurred. The opinions and comments from interview participants have not been verified, and these statements may contain erroneous or incomplete information.

Discussions were held with three individuals with experience at Bldg. 7500 ranging from 1987 through 2013. All three individuals have served in the capacity of Facility Manager. All three continue to provide support to ORNL facilities.

\section{INTERVIEWS}

One individual was the Facility Manager from 1987-1997. He stated that the underground storage tank (7562) was removed due to leaks that caused it to overflow. The tank was remediated and pulled.

Another individual served as the Surveillance and Maintenance (S\&M) Engineer from 1993 to 1995, then as the Facility Manager until 2000. There was a scale model of the building enclosed in a case that remained in the building. He states that this model could be useful when the facility is torn down. He heard that sometime during the operational period contamination was found in the bottom of a pit after a chemical operator who had been down in the pit tracked contamination from the pit to the nearest shower. Another incident occurred when he was removing papers from a desk drawer and a dried out dead mouse rolled over his hand, contaminating his hand.

The current Facility Manager has served in this capacity since 2008. He stated that the entire facility is a contamination area, requiring a Radiological Work Permit (RWP) and dress out before entry into the facility. The facility also has an asbestos area that requires respirator protection to enter the building, due to deteriorating pipe insulation. The building is almost unsafe to enter due to asbestos contamination. The roof of the facility is in poor condition and it leaks. The inside of the facility has mold and mildew issues. The facility has a north and south basement, each with approximately 5-ft of standing water. The water is contaminated with asbestos and radiological contamination. Periodically, water is pumped out of the basements. The equipment gravel area on the east side of the facility is contaminated. The area is posted as a contamination area (CA). The concrete under the shed on the northeast side of the facility is contaminated. The area is posted as a Radioactive Material Area (RMA). The contamination is mostly from by-products of uranium/fission, primarily, strontium and cesium. The facility is cold and dark. There is power going to only two pumps in the facility. 
Record of Communication: Building 7500, Bruce Howard

Project: ORAUIORISE ORN Process Inventory Waste Project

Subject: LIVng memory of hstorical coerations and waste management practices within Bulding 7500 at the Oak Ridge National Laboratory

\begin{tabular}{|l|l|l|}
\hline Intervew9: & Intervew Date: & Intervewer: \\
Eruce H. Howard & September 19,2013 & Unda Cottey \\
\hline
\end{tabular}

Role and Involvement with Bullding 7500

Late 2008 - present (Facilty Manager)

As Facity Manager, Mr. Howaro's involvement with Bulding 7500 incudes quarterty inspections to ensure that the facility is secure, checks for changing faclity condtions, emergency lighting, leakage, and that vegetation is controlied around the faclify.

\section{Summary of Conversation}

- The entre facility is a contamination area, requiring a Radlological Work Permit (RWP) and cress out before entry into the faclity. The faclity also has an asbestos area that requires respirator protection to enter the bulding, due to detertoraing pipe insulation. The building is almost unsate to enter due to asbestos contamination.

- The roct of the facilty is in poor condtion and it leaks.

- The inside of the faclity has moid and mildew issues.

- The faclity has a north and south basement that has approximately 5 feet of standing water in them. The water is contaminated with 3 sbestos and radiologlcal contamination. Periodicaly, water is pumped out of the basemerts.

- The equipment gravel area on the east side of the faclity is contaminated. The area is posted x a Contamination Area (CA).

- The concrete under the shed on the northeast side of the faclity is cortaminated. The area is posted a Radlosctive Materia Area (FMA).

- Contamination in this facility is primarty from reactor products of uranium. It was an experiment where teey used moten sait. It only operated for a couple of years. The contamination is mostly from by-products of uraniumilission, primarly, strontlum and cestum.

- The faclity is cold and dar.

- There is power going to only two pumps in the faclity.

Intervewer. Linda A.
Coffey 
Record of Communication: Building 7500, Mark Ford

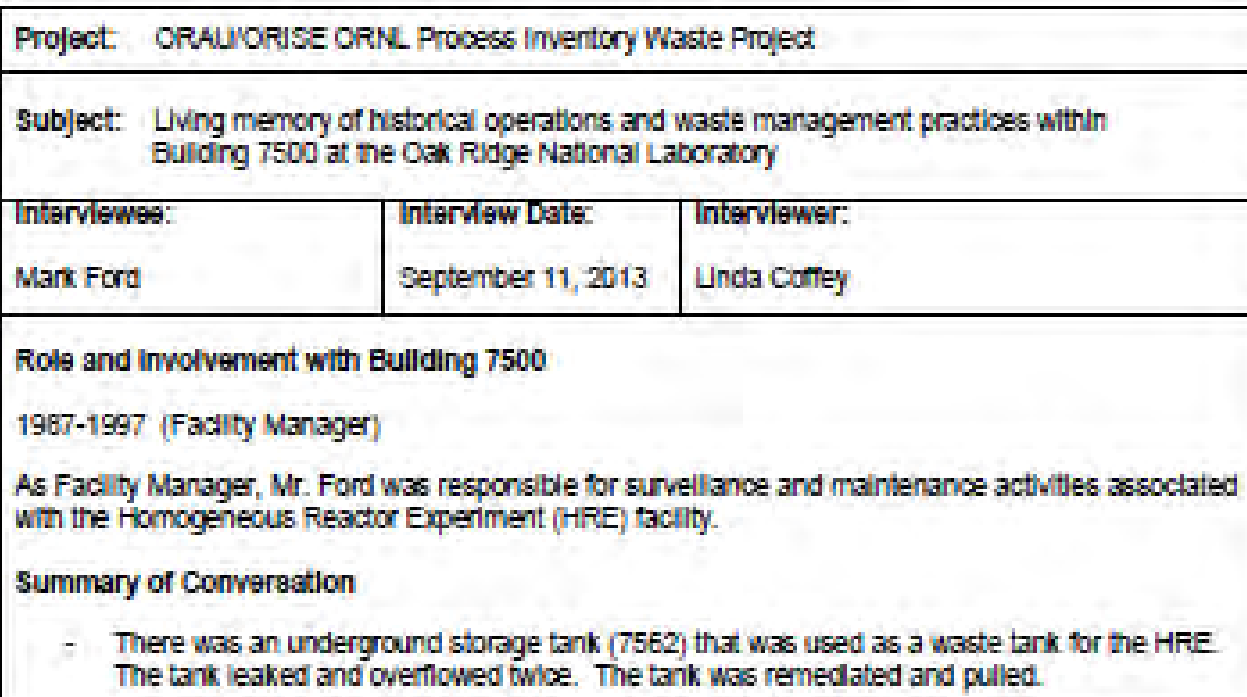
The tank leaked and overflowed twice. The tank was remedated and pulled.

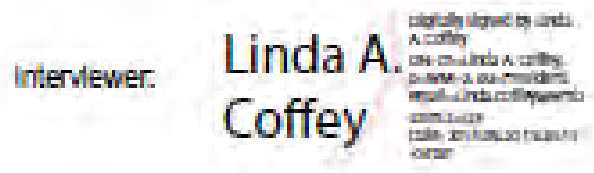




\section{Record of Communication: Building 7500, Timothy Cofer}

Project: ORALIORISE ORNL. Process inventory waste Project

Subject: Living memory of historical operations and waste management practices within Bulding 7500 at the Oak Ridge Nattonal Laboratory

\begin{tabular}{l|l|l}
\hline Intervewes: & Intervew Date: & Interviewer: \\
Timothy Cofer & September 11,2013 & Unda Cottey
\end{tabular}

Current Role: Mr. Coter currently has responsibility for the water distributon plant, the sewer plant, and the storm collecton system Oak Fuoge National Laboratory (OFNL).

Roie and Involvement with Bullding 7500

1993 - 1995 (Survelliance and Maintenance Facility Engineer)

1996 - 2000 (Faclity Manzger)

Summary of Conversation

- The faclity nas three storles, four are underground.

- There was a scale model of the building encosed in a case that was in the builing. This model cousd be usefu when the facility's tom down.

- Mr. Cofer heard that contaminaton was found in the bottom of a pit aner a chemical coerstor who had been down in the plt tracked contaminaton from tee pit to the nearest shower.

- There was an Incident when Mr. Cofer was removing pepers tom a desk drauer and a drled out, dead mouse rolled over his hand, contaminating his hand.

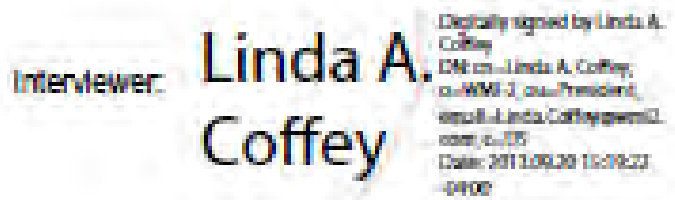


APPENDIX F

HAZARDOUS MATERIALS OF CONCERN 


\section{ORAU/ORISE \\ Process Inventory Waste Project}

\section{Chemical Contamination and Hazardous Materials of Construction ORNL Building 3001}

September 2013

Prepared by

Waste Management Innovations, Inc.

305 S. Chamberlain Avenue

Rockwood, TN 37854

865-776-5041 


\section{INTRODUCTION}

The purpose of this document is to provide an overview of the types and extent of chemical contamination and hazardous materials of concern that were observed during a recent tour of the facility. Information was also obtained by reviewing existing facility specific documentation and through discussion with the Facility Manager.

\section{CHEMICAL CONTAMINAITON}

Historical Chemical Use: Demineralizer utilized ion exchange resins (UCOR 2013).

Chemical Storage: Demineralizer resins have been removed. Process fluids and chemicals have been removed from this facility (UCOR 2013). Current hazardous materials inventory limited to small quantities of household cleaning solutions. No additional hazardous chemicals exceeding their respective 40 CFR 302.4 Reportable Quantity (RQ) will be introduced. Procedures are in place to ensure that the Facility Manger approves all activities, including those involving an increase in the radiological or chemical materials inventory (UCOR 2013).

Waste Accumulation: There was no evidence of speculative accumulation of any items that require additional characterization for chemical constituents or management as RCRA or TSCA regulated waste.

The CERCLA waste management area near the canal entrance containing "legacy waste” was observed and found to contain non-hazardous and rad waste only.

All RCRA containing constituents, including an estimated 275 gallons of sludge (0.5 inches) were removed from the canal as part of the RCRA closure of the canal in 1992 (UCOR 2013).

Beryllium Contamination: Historical literature does not indicate any use or storage of beryllium at this facility.

Chemical Contamination of Process Systems: The process systems have all been drained and the process fluids and chemicals have been removed from this facility (UCOR 2013). However, residual quantities of resin may still be present in ion exchange columns. The demineralizer room was not included on the tour.

The specific chemicals used in Building 3001 are not known. The waste stream from D\&D of this building will not exhibit the characteristic of ignitability if all traces of ignitable liquids and oxidizing solutions absorbed into the process equipment components and the resulting waste stream is solid debris. Additionally, the waste stream from D\&D of this building will not exhibit the characteristic of corrosivity if all traces of corrosive liquids have been absorbed into the process equipment components and the resulting waste stream is solid debris. 
The EPA has provided a regulatory clarification that the F001-listing is only appropriate when the listed solvents are used in a large-scale degreasing operation such as cold cleaning or vapor degreasing on an industrial scale. Large-scale degreasing operations were not identified in the Research Reactor facilities. Therefore, debris from D\&D of Bldg. 3001 will not carry the EPA hazardous waste number F001.

The F003-listed solvents acetone, methanol, and xylene could have potentially been used in this facility and may be present as contaminants of the process systems. However, F003-listed waste is listed solely for ignitability, and the waste stream from D\&D of this building will not exhibit the characteristic of ignitability if all traces of solvent residues were absorbed into the process equipment components and the resulting waste stream is solid debris. Therefore, the process equipment from this building will not meet the definition of an F003-listed hazardous waste.

The operations conducted by the Research Reactor facilities did not contain hazardous waste from any of the specific sources in 40 CFR 261.32, and therefore, any debris generated during D\&D of this facility will not be a K-listed waste.

\section{HAZARDOUS MATERIALS OF CONSTRUCTION}

Hood / Hot Cell Materials of Construction Concerns: The graphite pile / core of the reactor remains intact in the National Historic Monument museum portion of the facility.

Lead Bricks / Shielding: None encountered

Facility Infrastructure (Hoists, Cranes, Elevators): Facility infrastructure (hoists, cranes, elevators): 2 ton crane housed in room 110A (no visible oil stains on crane or concrete floor below). The facility crane is out of service (UCOR 2013).

PCBs: End point of duct posted 'Caution PCB Contaminated Gaskets'; located near the NW corner of the reactor's concrete enclosure on $1^{\text {st }}$ floor; this section of duct has been abandoned in place and is sealed shut with plywood; no visible stains or evidence of residue leaking from gasket.

The majority of mercury vapor, sodium vapor, and fluorescent lighting fixtures installed at this facility are equipped with ballasts that contain small power correction capacitors. The ballasts manufactured before 1979 contain dielectric fluid consisting of pure PCBs, typically defined as concentrations $>9,000 \mathrm{ppm}$. Fluorescent lighting ballast contains roughly one ounce of dielectric fluid, and each fluorescent fixture is typically equipped with two ballasts. The mercury vapor and sodium vapor lighting fixtures have one large ballast equipped with 2 or more three ounce capacitors. 
Thermostats and/or Mercury Switches: Multiple wall mounted thermostats observed near chillers on first floor; rooms on north end of second floor equipped with window unit air conditioners which have internal thermostats.

Refrigerants: All chillers, window unit a/c, and drinking fountains in this building are active with fully charged compressors.

Asbestos-Containing Material (ACM): Asbestos insulation on the steam pipes in Building 3001 was not removed during facility deactivation activities. There are also asbestos containing linoleum floor tiles (UCOR 2013). Pipe insulation throughout building is clearly labeled as ACM and appears to be in good condition; Floor tile also observed to be in good condition.

Lead Based Paint: The interior walls of Building 3001 are painted with lead-based paint. The lead in the paint is chemically bound and unavailable for release (UCOR 2013); Paint appears intact on interior structural material, wall surfaces, and concrete floors; slight evidence of peeling observed on exterior corrugated metal walls.

Light Bulbs: The mercury vapor, sodium vapor, fluorescent, and incandescent bulbs installed throughout this building were manufactured with toxic level of heavy metals. When subject to the TCLP, the mercury vapor and fluorescent bulbs will exceed the toxicity limits established for mercury, and sodium vapor and incandescent bulbs will exceed limits established for lead.

\section{REFERENCES}

UCOR, July 2013, Preliminary Hazard Screening for Building 3001, Oak Ridge Graphite Reactor, and Support Buildings (3002, 3003, and 3018) Oak Ridge National Laboratory, Oak Ridge Tennessee, PHS-OR-OGR-0998/Rev. 2. 


\section{ORAU/ORISE \\ Process Inventory Waste Project}

\section{Chemical Contamination and Hazardous Materials of Construction \\ ORNL Building 3005}

September 2013

Prepared by

Waste Management Innovations, Inc.

305 S. Chamberlain Avenue

Rockwood, TN 37854

865-776-5041 


\section{INTRODUCTION}

The purpose of this document is to provide an overview of the types and extent of chemical contamination and hazardous materials of concern that are assumed to be present based on the age and history of this facility. Information was obtained by reviewing existing facility specific documentation and through discussion with the Facility Manager.

\section{CHEMICAL CONTAMINAITON}

Historical Chemical Use: The Low Intensity Test Reactor (LITR) at Bldg. 3005 was converted from a hydraulic mockup of materials testing reactor to an operating reactor for the purpose of supplying a variety of irradiation facilities for ORNL and other research groups (Ford and Holder 1992). This facility did not house or involve operations that used production quantities of process chemicals.

Chemical Storage: Building 3005 is one of the ORNL facilities classified as "Other Industrial" which indicates a low quantity of hazardous chemical inventory (UCOR 2013).

Waste Accumulation: The limited access to this facility prevented a tour of the waste accumulation areas. The facility manager (FM) for Building 3005 is also responsible for Buildings 3001, 3010, and 3042, and the waste accumulation areas of the other facilities were consistently well maintained with no evidence of speculative accumulation of RCRA or TSCA regulated waste.

Beryllium Contamination: The LITR was a water-moderated and water-cooled reactor that used beryllium as a reflector until it was shut down in 1968. At that time, all fuel and shim-safety rods were removed, the water was drained. The space around the LITR defueled reactor core is filled to a height of 38 in. with solid blocks of 2-in, by 2-in, by 8-in, beryllium reflector. The beryllium is in a solid form. The thickness of this layer is at least $8 \mathrm{in}$. and is thicker on the north and south sides where it filled the void completely to the reactor tank shell. The estimated quantity of beryllium is $4500 \mathrm{lb}$. The beryllium is not considered releasable in its current form (2013).

Chemical Contamination of Process Systems: The specific chemicals used in Building 3005 are not known. The waste stream from D\&D of this building will not exhibit the characteristic of ignitability if all traces of ignitable liquids and oxidizing solutions absorbed into the process equipment components and the resulting waste stream is solid debris. Additionally, the waste stream from D\&D of this building will not exhibit the characteristic of corrosivity if all traces of corrosive liquids have been absorbed into the process equipment components and the resulting waste stream is solid debris.

The EPA has provided a regulatory clarification that the F001-listing is only appropriate when the listed solvents are used in a large-scale degreasing operation such as cold cleaning or vapor degreasing on an industrial scale. Large-scale degreasing operations were not identified in the 
Research Reactor facilities. Therefore, debris from D\&D of Bldg. 3005 will not carry the EPA hazardous waste number F001.

The F003-listed solvents acetone, methanol, and xylene could have potentially been used in this facility and may be present as contaminants of the process systems. However, F003-listed waste is listed solely for ignitability, and the waste stream from D\&D of this building will not exhibit the characteristic of ignitability if all traces of solvent residues were absorbed into the process equipment components and the resulting waste stream is solid debris. Therefore, the process equipment from this building will not meet the definition of an F003-listed hazardous waste.

The operations conducted by the Research Reactor facilities did not contain hazardous waste from any of the specific sources in 40 CFR 261.32, and therefore, any debris generated during D\&D of this facility will not be a K-listed waste.

\section{HAZARDOUS MATERIALS OF CONSTRUCTION}

Hood / Hot Cell Materials of Construction Concerns: The LITR was removed from service in 1968. At that time, all fuel and shim-safety rods were removed, the water was drained. The only toxic materials identified in the LITR are lead. The lead bricks are stacked around the reactor vessel exterior and over the top for the purposes of shielding. The lead over the top is also in cloth bags. Several of the bags have broken open, and the lead shot pellets are lying around loose. There is additional bulk lead shielding over piping throughout the facility. The estimated lead mass is approximately 47,000 lb. Lead shielding is not considered releasable in its current form (UCOR 2013).

Lead Bricks / Shielding: The use of bulk lead for shielding purposes is discussed in the Hood / Hot Cell Materials of Construction Concerns section.

Facility Infrastructure (Hoists, Cranes, Elevators): This building was not specifically inspected for the presence of hoists and cranes.

PCBs: Building 3005 was constructed in 1951. The majority of mercury vapor, sodium vapor, and fluorescent lighting fixtures installed at ORNL facilities of similar age are equipped with ballasts that contain small power correction capacitors. Ballasts manufactured before 1979 contain dielectric fluid consisting of pure PCBs, typically defined as concentrations $>9,000 \mathrm{ppm}$. Fluorescent lighting ballast contains roughly one ounce of dielectric fluid, and each fluorescent fixture is typically equipped with two ballasts. The mercury vapor and sodium vapor lighting fixtures have one large ballast equipped with 2 or more three ounce capacitors. 
This building was not specifically inspected for the presence of light fixtures that may contain PCB ballasts. However, any of the light fixtures described in this section are assumed to contain PCB capacitors and should be managed in accordance with the facility specific waste handling plan when the facility becomes available for demolition.

Thermostats and/or Mercury Switches: This building was not specifically inspected for the presence of window unit air conditioners $(\mathrm{a} / \mathrm{c})$, wall mounted thermostats, or fire alarm pull boxes. However, all of these devices contain mercury and if encountered, should be removed from the facility prior to the initiation of demolition activities.

Refrigerants: This building was not specifically inspected for the presence of chillers, window unit $\mathrm{a} / \mathrm{c}$, or drinking fountains. Any of these devices remaining in this building are assumed to still contain refrigerants and oil in the compressors and should be removed from the facility prior to the initiation of demolition activities.

Asbestos-Containing Material (ACM): This building was not specifically inspected for the presence ACM. Documents containing estimates of the type and condition of ACM installed at Building 3005 or the results of previous asbestos surveys at this facility could not be located.

Lead Based Paint: The interior of Building 3005 is assumed to be painted with lead-based paint. The interior of this facility was not inspected to determine the condition of painted surfaces.

Light Bulbs: Building 3005 was constructed in 1951. The mercury vapor, sodium vapor, fluorescent, and incandescent bulbs installed in ORNL facilities of similar age were manufactured with toxic level of heavy metals. When subject to the TCLP, the mercury vapor and fluorescent bulbs will exceed the toxicity limits established for mercury, and sodium vapor and incandescent bulbs will exceed limits established for lead.

\section{REFERENCES}

UCOR, June 2013, Preliminary Hazard Screening for Miscellaneous Areas and S\&M Activities at ORNL, Oak Ridge Tennessee, PHS-OR-S\&M-1000, Rev. 10.

Ford, M.K. and L. Holder, Jr., July 1992. Surveillance and Maintenance Plan for the ORNL Decontamination and Decommissioning Program, FY 1993 - 2002, prepared by Waste Management and Remedial Action Division, Oak Ridge, Tennessee. 


\section{ORAU/ORISE \\ Process Inventory Waste Project}

\section{Chemical Contamination and Hazardous Materials of Construction ORNL Building 3010}

September 2013

Prepared by

Waste Management Innovations, Inc.

305 S. Chamberlain Avenue

Rockwood, TN 37854

865-776-5041 


\section{INTRODUCTION}

The purpose of this document is to provide an overview of the types and extent of chemical contamination and hazardous materials of concern that were observed during a recent tour of the facility. Information was also obtained by reviewing existing facility specific documentation and through discussion with the Facility Manager.

\section{CHEMICAL CONTAMINAITON}

Historical Chemical Use: This facility housed a demineralizer system which served the reactor pool. The Low Temperature Neutron Irradiation Facility (LTNIF) used helium. There is an active natural gas line (2 in diameter) that runs the length of Building 3010 on the eastern side (UCOR 2013).

Chemical Storage: Two nitrogen cylinders remain connected to evacuation alarm in reactor bay. The demineralizers have been removed from the building. Tank I3822 (500 gallon stainless steel tank to supply helium for Bldg. 3010) has been emptied of its contents and isolated from the building (UCOR 2013).

Waste Accumulation: Various rad contaminated items were found on the north end of the reactor bay floor under the stairs including a drum of 'Pool Filters Non-Hazardous' and a pool basket. An unmarked drum with yellow tape covering the bungs was observed through the window of the PCA control room. A CERCLA waste management area north of the reactor pool consisted of a B-25 box with the lid secured. With the exception of the unmarked drum, there was no evidence of speculative accumulation of any items that require additional characterization for chemical constituents or management as RCRA or TSCA regulated waste.

Beryllium Contamination: The reactor had a beryllium reflector consisting of a series of moveable beryllium oxide blocks (Breazeale 1951). The facility manager stated that the reflector is no longer in place. There is no evidence that storage of solid forms of beryllium in a reactor pool resulted in beryllium contamination of the high bay or any of the remaining components of the demineralizer system (piping, filters, pump).

Chemical Contamination of Process Systems: The specific chemicals used in Building 3010 are not known. The waste stream from D\&D of this building will not exhibit the characteristic of ignitability if all traces of ignitable liquids and oxidizing solutions absorbed into the process equipment components and the resulting waste stream is solid debris. Additionally, the waste stream from D\&D of this building will not exhibit the characteristic of corrosivity if all traces of corrosive liquids have been absorbed into the process equipment components and the resulting waste stream is solid debris. 
The EPA has provided a regulatory clarification that the F001-listing is only appropriate when the listed solvents are used in a large-scale degreasing operation such as cold cleaning or vapor degreasing on an industrial scale. Large-scale degreasing operations were not identified in the Research Reactor facilities. Therefore, debris from D\&D of Bldg. 3010 will not carry the EPA hazardous waste number F001.

The F003-listed solvents acetone, methanol, and xylene could have potentially been used in this facility and may be present as contaminants of the process systems. However, F003-listed waste is listed solely for ignitability, and the waste stream from D\&D of this building will not exhibit the characteristic of ignitability if all traces of solvent residues were absorbed into the process equipment components and the resulting waste stream is solid debris. Therefore, the process equipment from this building will not meet the definition of an F003-listed hazardous waste.

The operations conducted by the Research Reactor facilities did not contain hazardous waste from any of the specific sources in 40 CFR 261.32, and therefore, any debris generated during D\&D of this facility will not be a K-listed waste.

\section{HAZARDOUS MATERIALS OF CONSTRUCTION}

Hood / Hot Cell Materials of Construction Concerns: Several activated components are stored in the reactor pool which contains 130,000 gallons of water for shielding. The reactor control rods are still in place. They include 2 shim (i.e. safety) rods and 1 regulating rod described as cadmium-lead with a cross section of 1-1/4 in x 2-1/2 in (Breazeale 1951).

Lead Bricks / Shielding: Observed the following: two lead pigs (10 in dia x $3 \mathrm{ft}$ ) in the reactor bay; lead bricks have been removed from the reactor bay valve pit and are staged in the south annex.

Facility Infrastructure (Hoists, Cranes, Elevators): Two bridge cranes are installed in the high bay; a small crane is mounted in the south annex west end.

PCBs: The original fluorescent light fixtures were abandoned in place above the drop ceiling in the south annex control room (the set of fluorescent light fixtures installed in the dropped ceiling are still in service). The majority of mercury vapor, sodium vapor, and fluorescent lighting fixtures installed at this facility are equipped with ballasts that contain small power correction capacitors. The ballasts manufactured before 1979 contain dielectric fluid consisting of pure PCBs, typically defined as concentrations $>9,000 \mathrm{ppm}$. Fluorescent lighting ballast contains roughly one ounce of dielectric fluid, and each fluorescent fixture is typically equipped with two ballasts. The mercury vapor and sodium vapor lighting fixtures have one large ballast equipped with 2 or more three ounce capacitors.

Thermostats and/or Mercury Switches: Window unit air conditioners (a/c) typically contain internal thermostats. The control room of the south annex and the PCA control room at the reactor 
bay are equipped with window unit a/c units. Wall mounted thermostats were observed in the south annex. The office area was not inspected.

Refrigerants: All chillers, window unit a/c, and drinking fountains in this building are assumed to still contain refrigerants and oil in the compressors.

Asbestos-Containing Material (ACM): Reactor bay steam pipe insulation is not specifically labeled as ACM but is in good condition; floor tiles are in place on the floor of the reactor bay and were found to be in poor condition; the south annex floor tile is in fair condition; one piece of scrap equipment in the south annex was tagged as ACM.

Lead Based Paint: The interior of Building 3010 is assumed to be painted with lead-based paint. The painted surfaces of the reactor bay and annex were inspected during the walk down and mostly found to be in good condition with the exception of the exterior of the reactor pool walls.

Light Bulbs: The mercury vapor, sodium vapor, fluorescent, and incandescent bulbs installed throughout this building were manufactured with toxic level of heavy metals. When subject to the TCLP, the mercury vapor and fluorescent bulbs will exceed the toxicity limits established for mercury, and sodium vapor and incandescent bulbs will exceed limits established for lead.

\section{REFERENCES}

UCOR, July 2013, Preliminary Hazard Screening for Building 3001, Oak Ridge Graphite Reactor, and Support Buildings (3002, 3003, and 3018) Oak Ridge National Laboratory, Oak Ridge Tennessee, PHS-OR-OGR-0998/Rev. 2.

Breazeale, William M. 1951, The New Bulk Shielding facility at Oak Ridge National Laboratory, ORNL-991. 


\section{ORAU/ORISE \\ Process Inventory Waste Project}

\section{Chemical Contamination and Hazardous Materials of Construction \\ ORNL Building 3028}

September 2013

Prepared by

Waste Management Innovations, Inc.

305 S. Chamberlain Avenue

Rockwood, TN 37854

865-776-5041 


\section{INTRODUCTION}

The purpose of this document is to provide an overview of the types and extent of chemical contamination and hazardous materials of concern that were observed during a recent tour of the facility. Information was also obtained by reviewing existing facility specific documentation and through discussion with the Facility Manager.

\section{CHEMICAL CONTAMINAITON}

Historical Chemical Use: The curium source fabrication operations involved precipitating the curium solution.

Chemical Storage: No chemical storage observed. Minor quantities of chemicals that may be present (e.g., decontamination solutions, janitorial supplies, lubricants for motors, etc.) are standard industrial by nature (UCOR 2012).

Waste Accumulation: The majority of waste staged in this facility was observed in the east air lock and consisted of general trash (paper, plastic, PPE). The access area to cells 1-5 contained several wood ladders, hand tools, bags of PPE, and extra prefilters for the chiller. Even though the housekeeping in this facility was poor and various items had been abandoned inside the rad areas, there was no evidence of speculative accumulation of any items that require additional characterization for chemical constituents or management as RCRA or TSCA regulated waste.

Beryllium Contamination: One of the operations conducted by the Isotopes Programs facilities was the fabrication of neutron radiation sources using beryllium powder and $\mathrm{Am}-241, \mathrm{Pu}-238$, or Pu-239 oxide. However, there is no mention throughout the historical literature of Building 3028 being one of the facilities utilized for this type of work or any other use or storage of beryllium (CCP-AKORNL-008).

Chemical Contamination of Process Systems: Ignitable liquids used in the Isotopes Programs facilities included acetone, benzene, diethylbenzene, dodecane, isopropyl alcohol, methanol, toluene, xylene, and several commercial products. Solutions containing oxidizing compounds were also used in the Isotopes Programs facilities, including aluminum nitrate, ammonium peroxysulfate, ammonium nitrate, hydrogen peroxide, hydroxylamine nitrate, potassium permanganate, and sodium nitrite (CCP-AK-ORNL-008). The specific chemicals used in Building 3028 are not known. The waste stream from D\&D of this building will not exhibit the characteristic of ignitability if all traces of ignitable liquids and oxidizing solutions absorbed into the process equipment components and the resulting waste stream is solid debris. 


\section{Chemical Contamination/Hazardous Materials of Construction \\ ORNL Building 3028}

September 2013

Corrosive liquids used in the Isotopes Programs facilities included acids (e.g., acetic, formic, hydrochloric, hydrofluoric, nitric, oxalic, sulfuric, sulfurous, and trichloroacetic) and caustics (e.g., ammonium hydroxide, sodium hydroxide) (CCP-AK-ORNL-008). The specific corrosive chemicals used in Building 3028 are not known. However, the waste stream from D\&D of this building will not exhibit the characteristic of corrosivity if all traces of corrosive liquids have been absorbed into the process equipment components and the resulting waste stream is solid debris.

There is the potential that U-listed chemicals may have been used for cleaning, as a dissolution agent, or as an eluent in this facility. As a result, portions of the related process equipment (hoods, glove boxes, piping, ventilation ducts, filter media, etc.) may be contaminated with residuals of these chemicals. Debris from D\&D of these process systems would not meet the definition of a U-listed waste if the original U-listed chemicals were used as sole-active ingredients for their intended purpose.

The EPA has provided a regulatory clarification that the F001-listing is only appropriate when the listed solvents are used in a large-scale degreasing operation such as cold cleaning or vapor degreasing on an industrial scale. Large-scale degreasing operations were not identified in the Isotopes Programs facilities. Therefore, debris from D\&D of Bldg. 3028 will not carry the EPA hazardous waste number F001.

The F003-listed solvents acetone, methanol, and xylene could have potentially been used during this facility's chemical processing operations and may be present as contaminants of the process systems. However, F003-listed waste is listed solely for ignitability, and the waste stream from D\&D of this building will not exhibit the characteristic of ignitability if all traces of solvent residues were absorbed into the process equipment components and the resulting waste stream is solid debris. Therefore, the process equipment from this building will not meet the definition of an F003-listed hazardous waste.

The operations conducted by the Isotopes Program facilities did not contain hazardous waste from any of the specific sources in 40 CFR 261.32, and therefore, any debris generated during D\&D of this facility will not be a K-listed waste.

\section{HAZARDOUS MATERIALS OF CONSTRUCTION}

Hood / Hot Cell Materials of Construction Concerns: The Short Lived Fission Product Facility on the west end of the building contains Cell 7. The shielding for cell 7 includes a 1-ft east wall lined with 4 in. of lead; an armor-plate, lead-lined steel access port in the west wall; and two lead-glass, mineral-oil-filled windows. Replacement of the windows was partially completed (during the 80s?) and they now have only a single pane of glass (UCOR 2012). 
Curium source fabrication and Alpha powder handling operations were conducted at the east end of Bldg. 3038 in Cells 1, 2, 3, 4, 5. Cells 1 - 4 are manipulator cells with one observation window each. The water-filled cell windows for Cells 1-4 have been drained. Cell 5 has a lead-glass, oil filled window. The water shield tanks at the top of each cell are now drained.

The center portion of the building houses Cell 6 which was originally used for production of ${ }^{147} \mathrm{Pm}$. This cell has lead shielding along the east wall; steel-clad-lead access doors in the north and east walls. Cell 6 is a process cell and has no window (UCOR 2012).

The hot cells were typically equipped with a combination of incandescent spot lights and mercury vapor lamps which are conservatively assumed to still be in place.

Lead Bricks / Shielding: Solid lead is present as bulk shielding material of hot cells and was discussed in the Hood / hot cell materials of concern section.

Facility Infrastructure (Hoists, Cranes, Elevators): Two small hoists present in west air lock; large crane installed on the north east end of the access area for cells 1-4.

PCBs: Transformers and large pieces of equipment containing greater than $50 \mathrm{ppm}$ PCBs were not identified in any of the Isotopes Program facilities (CCP-AK-ORNL-008, 2010).

The majority of mercury vapor, sodium vapor, and fluorescent lighting fixtures installed at this facility are equipped with ballasts that contain small power correction capacitors. The ballasts manufactured before 1979 contain dielectric fluid consisting of pure PCBs, typically defined as concentrations $>9,000 \mathrm{ppm}$. Fluorescent lighting ballast contains roughly one ounce of dielectric fluid, and each fluorescent fixture is typically equipped with two ballasts. The mercury vapor and sodium vapor lighting fixtures have one large ballast equipped with 2 or more three ounce capacitors.

Thermostats and/or Mercury Switches: Wall mounted thermostats present at powder cell access area to control chiller.

Refrigerants: All chillers in this building are assumed to still contain refrigerants and oil in the compressors.

Asbestos-Containing Material (ACM): Steam piping throughout the facility is labeled as ACM and was observed to be in good condition. The Facility Manager estimates that there are approximately $5000 \mathrm{ft}^{3}$ of facility structures containing asbestos in the form of resilient floor tiles, sheet vinyl flooring, roof felts, asphalt tiles, transite roofing shingles, transite siding, and transite pipe (UCOR 2012). 
Lead Based Paint: The interior of Building 3028 is painted with lead-based paint. The painted surfaces were inspected during the walk down and mostly found to be in good condition with limited peeling/chipping. The paint on the concrete floor of the east air lock is peeling/cracking.

Light Bulbs: The mercury vapor, sodium vapor, fluorescent, and incandescent bulbs installed throughout this building were manufactured with toxic level of heavy metals. When subject to the TCLP, the mercury vapor and fluorescent bulbs will exceed the toxicity limits established for mercury, and sodium vapor and incandescent bulbs will exceed limits established for lead.

\section{REFERENCES}

UCOR April 2012, Hazard Assessment Document for Building 3028, Oak Ridge National Laboratory, Oak Ridge Tennessee, HAD-OR-3028-0058, Rev. 3.

CCP-AK-ORNL-008, Central Characterization Project Acceptable Knowledge Summary Report for Oak Ridge National Laboratory Isotopes Programs Contact-Handled Transuranic Wastes, July 06, 2010 (no author sited). 


\section{ORAU/ORISE \\ Process Inventory Waste Project}

\section{Chemical Contamination and Hazardous Materials of Construction \\ ORNL Building 3029}

September 2013

Prepared by

Waste Management Innovations, Inc.

305 S. Chamberlain Avenue

Rockwood, TN 37854

865-776-5041 


\section{INTRODUCTION}

The purpose of this document is to provide an overview of the types and extent of chemical contamination and hazardous materials of concern that were observed during a recent tour of the facility. Information was also obtained by reviewing existing facility specific documentation and through discussion with the Facility Manager.

\section{CHEMICAL CONTAMINAITON}

Historical Chemical Use: Operations in this facility included chemical processing of radioactive materials. Cell 4 was used for chemical processing of short half-life materials (UCOR 2011).

Chemical Storage: None observed. Minor quantities of chemicals that may be present (e.g., decontamination solutions, janitorial supplies, lubricants for motors, etc.) are standard industrial by nature (UCOR 2011).

Waste Accumulation: The abandoned / discarded items in this facility included the following: a 16gallon drum in the center lab labeled 'Returned from SMO' and 'Cell 2'; two empty 5-gallon drums previously used as shipping containers staged in the north foyer; a gallon container of floor cleaner staged in the north foyer; a 55-gallon drum labeled 'Used Oil' staged in the north foyer. With the exception of the used oil, there was no evidence of speculative accumulation of any items that require additional characterization for chemical constituents or management as RCRA or TSCA regulated waste.

Beryllium Contamination: One of the operations conducted by the Isotopes Programs facilities was the fabrication of neutron radiation sources using beryllium powder and Am-241, Pu-238, or Pu-239 oxide. However, there is no mention throughout the historical literature of Building 3029 being one of the facilities utilized for this type of work or any other use or storage of beryllium (CCP-AKORNL-008).

Chemical Contamination of Process Systems: Ignitable liquids used in the Isotopes Programs facilities included acetone, benzene, diethylbenzene, dodecane, isopropyl alcohol, methanol, toluene, xylene, and several commercial products. Solutions containing oxidizing compounds were also used in the Isotopes Programs facilities, including aluminum nitrate, ammonium peroxysulfate, ammonium nitrate, hydrogen peroxide, hydroxylamine nitrate, potassium permanganate, and sodium nitrite (CCP-AK-ORNL-008). The specific chemicals used in Building 3029 are not known. The waste stream from D\&D of this building will not exhibit the characteristic of ignitability if all traces of ignitable liquids and oxidizing solutions absorbed into the process equipment components and the resulting waste stream is solid debris. 


\section{Chemical Contamination/Hazardous Materials of Construction \\ ORNL Building 3029}

September 2013

Corrosive liquids used in the Isotopes Programs facilities included acids (e.g., acetic, formic, hydrochloric, hydrofluoric, nitric, oxalic, sulfuric, sulfurous, and trichloroacetic) and caustics (e.g., ammonium hydroxide, sodium hydroxide) (CCP-AK-ORNL-008). The specific corrosive chemicals used in Building 3029 are not known. However, the waste stream from D\&D of this building will not exhibit the characteristic of corrosivity if all traces of corrosive liquids have been absorbed into the process equipment components and the resulting waste stream is solid debris.

There is the potential that U-listed chemicals may have been used for cleaning, as a dissolution agent, or as an eluent in this facility. As a result, portions of the related process equipment (hoods, glove boxes, piping, ventilation ducts, filter media, etc.) may be contaminated with residuals of these chemicals. Debris from D\&D of these process systems would not meet the definition of a U-listed waste if the original U-listed chemicals were used as sole-active ingredients for their intended purpose.

The EPA has provided a regulatory clarification that the F001-listing is only appropriate when the listed solvents are used in a large-scale degreasing operation such as cold cleaning or vapor degreasing on an industrial scale. Large-scale degreasing operations were not identified in the Isotopes Programs facilities. Therefore, debris from D\&D of Bldg. 3029 will not carry the EPA hazardous waste number F001.

The F003-listed solvents acetone, methanol, and xylene could have potentially been used during this facility's chemical processing operations and may be present as contaminants of the process systems. However, F003-listed waste is listed solely for ignitability, and the waste stream from D\&D of this building will not exhibit the characteristic of ignitability if all traces of solvent residues were absorbed into the process equipment components and the resulting waste stream is solid debris. Therefore, the process equipment from this building will not meet the definition of an F003-listed hazardous waste.

The operations conducted by the Isotopes Program facilities did not contain hazardous waste from any of the specific sources in 40 CFR 261.32, and therefore, any debris generated during D\&D of this facility will not be a K-listed waste.

\section{HAZARDOUS MATERIALS OF CONSTRUCTION}

Hood / Hot Cell Materials of Construction Concerns: This facility contains four manipulator hot cells, two laboratory hoods that could be converted to gloveboxes, and two small gloveboxes, known

as the ${ }^{99}$ Tc glovebox and the ${ }^{14} \mathrm{C}$ glovebox. Cell 2 has 2 inches of lead shielding. The access door on the back of Cell 2 is constructed of 8-inch armor plate plus 1 inch of lead. Cells 1,3 and 4 have shielded compartments for storing radioactive materials. In cells 1 and 3 , the materials can be stored in a stainless steel cylinder submerged in the operating pan and covered with a stainless steel clad 


\section{Chemical Contamination/Hazardous Materials of Construction \\ ORNL Building 3029}

September 2013

lead plug approximately 10.5 inches thick. The submerged portion of the well is surrounded by concrete and lead. In Cell 4, radioactive material can be stored in a shielded barricade projecting from the rear wall (UCOR 2011).

The hot cells were typically equipped with a combination of incandescent spot lights and mercury vapor lamps which are conservatively assumed to still be in place.

The cobalt garden is located below grade and may be outside the D\&D scope. The following information was obtained from the Preliminary Hazard Screening document for Bldg. 3029 and is presented for information purposes only.

The ${ }^{60}$ Co Garden is located behind Cell 2 in the cell charging area. The garden consists of 92 stationary stainless steel tubes surrounding a $1 \mathrm{ft}$ square, $7 \mathrm{ft}$ long irradiation plug. Each of the 92 tubes projects downward into a free space of shielding and terminates 1 foot above the floor of the excavation. Removable ${ }^{60}$ Co storage containers rested at the bottom of each of the tubes to provide a high gamma-ray flux to the irradiator plug at a location near the bottom of the facility. These containers are 1.25 inch diameter by 3.25 inches long stainless steel tubes closed with threaded chrome plated steel caps. Each of the 92 stationary tubes, which contain a ${ }^{60} \mathrm{Co}$ storage container, is plugged with an 11-7/8 inch long lead-filled stainless steel tube. All of the tubes are covered with 22 inches of lead shot at the top.

The $1 \mathrm{ft}$ square irradiator plug contains a specimen irradiation chamber at the bottom to which is attached a $1 \mathrm{ft}^{3}$ piece of lead. The location of the ${ }^{60} \mathrm{Co}$ storage containers is at a level with the irradiation chamber to provide a maximum gamma-ray flux to specimens in the chamber. The attached lead shield at the bottom of the irradiation plug protects operating personnel during loading and unloading of specimens. The portion of the irradiator plug above the specimen irradiation chamber is filled with concrete, which is penetrated by two quarter inch outer diameter tubes for introducing thermocouples or to provide a gas flow into the irradiation chamber. The centering device for positioning the shield over the proper stationary tube is a flat circular plate with a raised outer edge and with an inside diameter slightly greater than the overall diameter of the transfer shield. A short tube in the center of the plate projects downward to engage the top section of the hole in the stationary tube.

The entire ${ }^{60} \mathrm{Co}$ Garden is shielded with 22 inches of lead brick and 22 inches of barytes concrete. A stainless steel plate covers the entire garden (UCOR 2011).

Lead Bricks / Shielding: The following items were observed during the facility tour: two lead pigs (4 in dia x 6 in) near door to east lab; three lead pigs (4 in dia x 6 in) stored in cabinet along the lower south wall of the west lab. 
Facility Infrastructure (Hoists, Cranes, Elevators): Cells 1 and 2 are equipped with a hoist rated at 1-ton capacity (UCOR 2011).

PCBs: Transformers and large pieces of equipment containing greater than $50 \mathrm{ppm}$ PCBs were not identified in any of the Isotopes Program facilities (CCP-AK-ORNL-008, 2010).

The majority of mercury vapor, sodium vapor, and fluorescent lighting fixtures installed at this facility are equipped with ballasts that contain small power correction capacitors. The ballasts manufactured before 1979 contain dielectric fluid consisting of pure PCBs, typically defined as concentrations $>9,000 \mathrm{ppm}$. Fluorescent lighting ballast contains roughly one ounce of dielectric fluid, and each fluorescent fixture is typically equipped with two ballasts. The mercury vapor and sodium vapor lighting fixtures have one large ballast equipped with 2 or more three ounce capacitors.

Thermostats and/or Mercury Switches: Wall mounted thermostats present at each lab to control chillers.

Refrigerants: All chillers in this building are assumed to still contain refrigerants and oil in the compressors.

Asbestos-Containing Material (ACM): Portions of steam pipe insulation on the north side of the facility have been replaced with a silver backed insulation material labeled 'Asbestos Free'. The remaining steam pipe insulation without silver backing is not labeled, is assumed to contain asbestos, and appears to be in a non-friable state. Floor tile in this facility was observed to be in fair to poor condition.

Lead Based Paint: Paint in this facility is actively peeling off all horizontal and vertical surfaces. All horizontal surfaces are now littered with paint chips.

Light Bulbs: The majority of lighting in this facility is provided by fluorescent light fixtures. The mercury vapor, sodium vapor, fluorescent, and incandescent bulbs installed throughout this building were manufactured with toxic level of heavy metals. When subject to the TCLP, the mercury vapor and fluorescent bulbs will exceed the toxicity limits established for mercury, and sodium vapor and incandescent bulbs will exceed limits established for lead.

\section{REFERENCES}

UCOR December 2011, Preliminary Hazard Screening for the Source Development Laboratory, Building 3029, PHS-OR-3029-1005, Rev. 2.

CCP-AK-ORNL-008, Central Characterization Project Acceptable Knowledge Summary Report for Oak Ridge National Laboratory Isotopes Programs Contact-Handled Transuranic Wastes, July 06, 2010 (no author sited). 
Chemical Contamination/Hazardous Materials of Construction

ORNL Building 3029

September 2013

Page 5 of 5 


\section{ORAU/ORISE \\ Process Inventory Waste Project}

\section{Chemical Contamination and Hazardous Materials of Construction \\ ORNL Building 3038}

September 2013

Prepared by

Waste Management Innovations, Inc.

305 S. Chamberlain Avenue

Rockwood, TN 37854

865-776-5041 


\section{INTRODUCTION}

The purpose of this document is to provide an overview of the types and extent of chemical contamination and hazardous materials of concern that are assumed to be present based on the age and history of this facility. Information was also obtained by reviewing existing facility specific documentation and through discussion with the Facility Manager.

\section{CHEMICAL CONTAMINAITON}

Historical Chemical Use: Building 3038 was used for the study of transuranium elements, fabrication of alpha- and neutron-emitting targets and sources, shipment of radioisotopes, and the study of chemical processes involving tracer level activities. The specific chemicals utilized in these activities are not known. Historical documents provide an overview of chemicals that were thought to be in use by members of the Isotopes Production Program. Additional discussion of potential chemical use in Bldg. 3038 is provided in the discussion of chemical contamination of process systems located at the end of this section.

Chemical Storage: This building was not available to be walked down. Access to this building was suspended due to roof construction. The facility manager (FM) for Building 3038 is also responsible for Buildings 3028, 3029, 4507, and 3517. Chemical storage at 3038 is assumed to be consistent with conditions found at other facilities under the control of this Facility Manager.

Waste Accumulation: The limited access to this facility prevented a tour of the waste accumulation areas. The facility manager (FM) for Building 3038 is also responsible for Buildings 3028, 3029, 4507, and 3517. Waste accumulation at 3038 is assumed to be consistent with conditions found at other facilities under the control of this Facility Manager. The waste accumulation areas of the other facilities were consistently well maintained with no evidence of speculative accumulation of RCRA or TSCA regulated waste.

Beryllium Contamination: One of the operations conducted at Bldg. 3038 was the fabrication and destructive testing of Pu-Be neutron sources. Sources were fabricated by combining plutonium oxide and beryllium powder in a metal capsule and sealed by inert gas welding. However, there is no mention of the specific area of the building used to conduct this work (CCP-AK-ORNL-008).

Chemical Contamination of Process Systems: Ignitable liquids used in the Isotopes Programs facilities included acetone, benzene, diethylbenzene, dodecane, isopropyl alcohol, methanol, toluene, xylene, and several commercial products. Solutions containing oxidizing compounds were also used in the Isotopes Programs facilities, including aluminum nitrate, ammonium peroxysulfate, ammonium nitrate, hydrogen peroxide, hydroxylamine nitrate, potassium permanganate, and sodium nitrite (CCP-AK-ORNL-008). The specific chemicals used in Building 3038 are not known. The waste 


\section{Chemical Contamination/Hazardous Materials of Construction \\ ORNL Building 3038}

September 2013

stream from D\&D of this building will not exhibit the characteristic of ignitability if all traces of ignitable liquids and oxidizing solutions absorbed into the process equipment components and the resulting waste stream is solid debris.

Corrosive liquids used in the Isotopes Programs facilities included acids (e.g., acetic, formic, hydrochloric, hydrofluoric, nitric, oxalic, sulfuric, sulfurous, and trichloroacetic) and caustics (e.g., ammonium hydroxide, sodium hydroxide) (CCP-AK-ORNL-008). The specific corrosive chemicals used in Building 3038 are not known. However, the waste stream from D\&D of this building will not exhibit the characteristic of corrosivity if all traces of corrosive liquids have been absorbed into the process equipment components and the resulting waste stream is solid debris.

There is the potential that U-listed chemicals may have been used for cleaning, as a dissolution agent, or as an eluent in this facility. As a result, portions of the related process equipment (hoods, glove boxes, piping, ventilation ducts, filter media, etc.) may be contaminated with residuals of these chemicals. Debris from D\&D of these process systems would not meet the definition of a U-listed waste if the original U-listed chemicals were used as sole-active ingredients for their intended purpose.

The EPA has provided a regulatory clarification that the F001-listing is only appropriate when the listed solvents are used in a large-scale degreasing operation such as cold cleaning or vapor degreasing on an industrial scale. Large-scale degreasing operations were not identified in the Isotopes Programs facilities. Therefore, debris from D\&D of Bldg. 3038 will not carry the EPA hazardous waste number F001.

The F003-listed solvents acetone, methanol, and xylene could have potentially been used during this facility's chemical processing operations and may be present as contaminants of the process systems. However, F003-listed waste is listed solely for ignitability, and the waste stream from D\&D of this building will not exhibit the characteristic of ignitability if all traces of solvent residues were absorbed into the process equipment components and the resulting waste stream is solid debris. Therefore, the process equipment from this building will not meet the definition of an F003-listed hazardous waste.

Benzene was identified in a Building 3038 plutonium processing procedure. A solution of thenoyltrifluoroacetone and benzene was used for plutonium extraction. As a result, portions of the related process equipment (hoods, glove boxes, piping, ventilation ducts, filter media, etc.) may be contaminated with residuals of these chemicals. The records were not specific enough to identify a specific area of the building where this operation took place so the presence of residual benzene could only be determined through sampling and analysis of potentially effected equipment / media that may still be in place. Since plutonium extraction is a solvent use, EPA hazardous waste number F005 
could be assigned to debris from D\&D of the effected process systems that failed to meet TCLP limits for benzene.

The operations conducted by the Isotopes Program facilities did not contain hazardous waste from any of the specific sources in 40 CFR 261.32, and therefore, any debris generated during D\&D of this facility will not be a K-listed waste.

\section{HAZARDOUS MATERIALS OF CONSTRUCTION}

Hood / Hot Cell Materials of Construction Concerns: The hot cells of this facility were not observed, and documentation containing details about the materials of construction were not available.

Lead Bricks / Shielding: Limited access to this facility prevented an inspection for lead bricks or the use of bulk lead as shielding.

Facility Infrastructure (Hoists, Cranes, Elevators): This building was not inspected for the presence of hoists and cranes.

PCBs: Transformers and large pieces of equipment containing greater than 50 ppm PCBs were not identified in any of the Isotopes Program facilities (CCP-AK-ORNL-008, 2010).

Building 3038 was constructed in 1950. The majority of mercury vapor, sodium vapor, and fluorescent lighting fixtures installed at ORNL facilities of similar age are equipped with ballasts that contain small power correction capacitors. Ballasts manufactured before 1979 contain dielectric fluid consisting of pure PCBs, typically defined as concentrations $>9,000 \mathrm{ppm}$. Fluorescent lighting ballast contains roughly one ounce of dielectric fluid, and each fluorescent fixture is typically equipped with two ballasts. The mercury vapor and sodium vapor lighting fixtures have one large ballast equipped with 2 or more three ounce capacitors.

This building was not specifically inspected for the presence of light fixtures that may contain PCB ballasts. However, any of the light fixtures described in this section are assumed to contain PCB capacitors and should be managed in accordance with the facility specific waste handling plan when the facility becomes available for demolition.

Thermostats and/or Mercury Switches: This building was not specifically inspected for the presence of window unit air conditioners $(\mathrm{a} / \mathrm{c})$, wall mounted thermostats, or fire alarm pull boxes. However, all of these devices contain mercury and if encountered, should be removed from the facility prior to the initiation of demolition activities.

Refrigerants: This building was not specifically inspected for the presence of chillers, window unit $\mathrm{a} / \mathrm{c}$, or drinking fountains. Any of these devices remaining in this building are assumed to still 
contain refrigerants and oil in the compressors and should be removed from the facility prior to the initiation of demolition activities.

Asbestos-Containing Material (ACM): This building was not specifically inspected for the presence ACM. Documents containing estimates of the type and condition of ACM installed at Building 3038 or the results of previous asbestos surveys at this facility could not be located.

Lead Based Paint: The interior of Building 3038 is assumed to be painted with lead-based paint. The interior of this facility was not inspected to determine the condition of painted surfaces.

Light Bulbs: Building 3038 was constructed in 1950. The mercury vapor, sodium vapor, fluorescent, and incandescent bulbs installed in ORNL facilities of similar age were manufactured with toxic level of heavy metals. When subject to the TCLP, the mercury vapor and fluorescent bulbs will exceed the toxicity limits established for mercury, and sodium vapor and incandescent bulbs will exceed limits established for lead.

\section{REFERENCES}

CCP-AK-ORNL-008, Central Characterization Project Acceptable Knowledge Summary Report for Oak Ridge National Laboratory Isotopes Programs Contact-Handled Transuranic Wastes, July 06, 2010 (no author sited). 


\section{ORAU/ORISE \\ Process Inventory Waste Project}

\section{Chemical Contamination and Hazardous Materials of Construction ORNL Building 3042}

September 2013

Prepared by

Waste Management Innovations, Inc.

305 S. Chamberlain Avenue

Rockwood, TN 37854

865-776-5041 


\section{INTRODUCTION}

The purpose of this document is to provide an overview of the types and extent of chemical contamination and hazardous materials of concern that were observed during a recent tour of the facility. Information was also obtained by reviewing existing facility specific documentation and through discussion with the Facility Manager.

\section{CHEMICAL CONTAMINAITON}

Historical Chemical Use: Demineralizer system in the basement used bulk quantities of ion exchange resins, nitric acid, and caustic solution (sodium hypochlorite).

Chemical Storage: Demineralizer system in basement has been taken out of service. Bulk quantities of ion exchange resins, nitric acid and caustic solution have been removed, but equipment remains in place. Active demineralized water makeup system in the east end of the second floor truck loading area contains $6.0 \mathrm{ft}^{3}$ (168 lbs) of granular activated charcoal and approximately $13.2 \mathrm{ft}^{3}$ of high purity mixed bed resin in demineralizer tanks. The resins in the active system are totally inert (UCOR 2010). No hazardous materials from outside the Bldg. 3042 footprint shall be introduced with the exception of small quantities of materials that are incidental to authorized S\&M activities such as cleaners, decontamination solutions, etc. (UCOR 2010).

Waste Accumulation: There was no evidence of speculative accumulation of any items that require additional characterization for chemical constituents or management as RCRA or TSCA regulated waste.

The CERCLA waste management area south of the reactor pool contained non-hazardous and rad waste. Waste in this area was packaged and labeled, and housekeeping in the area was good.

Beryllium Contamination: The reflector was composed of an arrangement of beryllium elements and was surrounded by $4 \mathrm{ft}$ of water in the reactor pool. All beryllium has been removed from the facility (UCOR 2010). There is no evidence that storage of solid forms of beryllium in a reactor pool resulted in beryllium contamination of the high bay or any of the remaining components of the demineralizer system (piping, filters, pump).

Chemical Contamination of Process Systems: Residual quantities of resin are present in ion exchange columns (16 $\mathrm{ft}^{3}$ Anthracite left per notes on demineralizers west of pool in basement). The demineralizer room(s) under directly under the reactor pool were not accessible for viewing.

The specific chemicals used in Building 3042 are not known. The waste stream from D\&D of this building will not exhibit the characteristic of ignitability if all traces of ignitable liquids and oxidizing solutions absorbed into the process equipment components and the resulting waste stream is solid 
debris. Additionally, the waste stream from D\&D of this building will not exhibit the characteristic of corrosivity if all traces of corrosive liquids have been absorbed into the process equipment components and the resulting waste stream is solid debris.

The EPA has provided a regulatory clarification that the F001-listing is only appropriate when the listed solvents are used in a large-scale degreasing operation such as cold cleaning or vapor degreasing on an industrial scale. Large-scale degreasing operations were not identified in the Research Reactor facilities. Therefore, debris from D\&D of Bldg. 3042 will not carry the EPA hazardous waste number F001.

The F003-listed solvents acetone, methanol, and xylene could have potentially been used in this facility and may be present as contaminants of the process systems. However, F003-listed waste is listed solely for ignitability, and the waste stream from D\&D of this building will not exhibit the characteristic of ignitability if all traces of solvent residues were absorbed into the process equipment components and the resulting waste stream is solid debris. Therefore, the process equipment from this building will not meet the definition of an F003-listed hazardous waste.

The operations conducted by the Research Reactor facilities did not contain hazardous waste from any of the specific sources in 40 CFR 261.32, and therefore, any debris generated during D\&D of this facility will not be a K-listed waste.

\section{HAZARDOUS MATERIALS OF CONSTRUCTION}

Hood / Hot Cell Materials of Construction Concerns: Two out-of service hot cells are located at the west end of the storage pool on the third floor. Both cells are equipped with oil filled viewing windows. Each cell contains light fixtures (typically mercury vapor lamps with ballasts incorporated into the body of the lamp).

Several activated components are stored in the reactor pool which contains 125,000 gallons of water for shielding. The reactor control rods are still in place. The poison sections of the twelve reactor control rods were made of cadmium.

Lead Bricks / Shielding: Lead bricks form shielding for components of basement demineralizer system.

Facility Infrastructure (Hoists, Cranes, Elevators): Building elevator uses hydraulic oil from a reservoir located in the basement (elevator is still in operation). An out-of-service diesel generator is located in a room attached to the north side of Bldg. 3042 (fuel and coolant have been drained from generator and diesel storage tank is marked "empty”). Banks of six 12-volt batteries are located in the diesel generator room and on the third floor west wall. A 20-ton bridge crane is provided in the reactor bay. 
PCBs: End point of duct posted 'Caution PCB Contaminated Gaskets'; located on north side of reactor pool on 2nd floor; this section of duct has been abandoned in place and is sealed shut with plywood; no visible stains or evidence of residue leaking from gasket.

The cables routed to cable trays near the basement ceiling contain PCBs (cable trays posted with 'PCB $<50$ ppm' stickers). The switchgear cabinets located in the basement may contain three phase cable terminators (pot heads) packed with PCB laden grease. Portions of paper insulated lead cable (PILC) contained inside the basement transformer and switchgear cabinets may be impregnated with PCB laden mineral oil. The PILC and pot heads in Bldg. 3042 have not been sampled and analyzed for PCBs.

The majority of mercury vapor, sodium vapor, and fluorescent lighting fixtures installed at this facility are equipped with ballasts that contain small power correction capacitors. The ballasts manufactured before 1979 contain dielectric fluid consisting of pure PCBs, typically defined as concentrations $>9,000 \mathrm{ppm}$. Fluorescent lighting ballast contains roughly one ounce of dielectric fluid, and each fluorescent fixture is typically equipped with two ballasts. The mercury vapor and sodium vapor lighting fixtures have one large ballast equipped with 2 or more three ounce capacitors.

Thermostats and/or Mercury Switches: Multiple wall mounted thermostats observed near chillers on first floor; rooms on north end of second floor equipped with window unit air conditioners which have internal thermostats.

Refrigerants: All chillers, window unit a/c, and drinking fountains in this building are assumed to still contain refrigerants and oil in the compressors.

Asbestos-Containing Material (ACM): Basement level steam pipe insulation and wall board are labeled as ACM and appear to be in good condition. The floor tile used throughout the building is in fair condition and is assumed to contain asbestos due to its size (8 in x 8 in) and date of installation. The control panels on the third floor and the switchgear and transformer cabinets in the basement could not be inspected during this study, but are thought to contain asbestos wiring.

Lead Based Paint: The interior of Building 3042 is assumed to be painted with lead-based paint. The painted surfaces were inspected during the walk down and mostly found to be in good condition with no evidence of peeling or chipping. Paint cracking was observed on the exterior walls of the reactor pool on the third floor and on the concrete floor near the south hot cell.

Light Bulbs: The mercury vapor, sodium vapor, fluorescent, and incandescent bulbs installed throughout this building were manufactured with toxic level of heavy metals. When subject to the TCLP, the mercury vapor and fluorescent bulbs will exceed the toxicity limits established for mercury, and sodium vapor and incandescent bulbs will exceed limits established for lead. 
Chemical Contamination/Hazardous Materials of Construction ORNL Building 3042

September 2013

\section{REFERENCES}

UCOR, May 2010, Hazard Assessment Document for the Oak Ridge Research Reactor Building 3042, Oak Ridge National Laboratory, Oak Ridge Tennessee, HAD-OR-3042-0030, Rev. 10. 


\section{ORAU/ORISE \\ Process Inventory Waste Project}

\section{Chemical Contamination and Hazardous Materials of Construction ORNL Building 3517}

September 2013

Prepared by

Waste Management Innovations, Inc.

305 S. Chamberlain Avenue

Rockwood, TN 37854

865-776-5041 


\section{INTRODUCTION}

The purpose of this document is to provide an overview of the types and extent of chemical contamination and hazardous materials of concern that were observed during a recent tour of the facility. Information was also obtained by reviewing existing facility specific documentation and through discussion with the Facility Manager.

\section{CHEMICAL CONTAMINAITON}

Historical Chemical Use: Operations at this facility involved the processing of radioactive source materials from fission product waste solutions resulting from uranium fuel element processing. The process cells contained an array of tanks, piping, and other process equipment that were used in various sequences depending on the feed material and the product emphasized. The aqueous feed solution containing mixed fission products was concentrated by evaporation, and the fission products were separated into groups of chemically related elements by organic precipitation or cocrystallization reactions. The concentrated fractions were processed by liquid extraction and by further precipitation and crystallization to obtain separated fission products of high purity.

A report from 1960 provided a general description of the process chemistry associated with facility operations. The following chemicals were included as an appendix to the report: sodium hydroxide, nitric acid, ammonium sulfate, sodium hypophosphite, sodium sulfate, ferric nitrate, alum, hydrazine, versene, D2 Ethyl Hexyl Phosphoric Acid, hydrogen peroxide, potassium permanganate, hydrochloric acid, ammonia, ammonium hydroxide, oxalic acid, cerium nitrate, and Amsco 125-82. Versene is an EDTA-based chelating agent that was used as a common decontamination solution in the form of 0.1 M sodium versenate. Amsco 125-82 was used as an organic solvent.

Chemical Storage: A phosphoric acid warning label was observed on the portion of the electropolisher located in the crane bay. Solid residue was observed on the crane bay floor in the vicinity of 1 in pipe run near the chemical warning label. The sodium hydroxide and nitric acid tanks at make-up area 31 on the second floor are thought to be empty but may contain heels of the chemicals. An argon line (may be part of an argon regulator system associated with the windows of the manipulator cells) runs along cell wall of the manipulator operating gallery but is marked 'out of service'. There was no evidence of on-going hazardous chemical storage at this facility.

Waste Accumulation: The majority of waste staged in this facility was observed in the crane bay and consisted of general trash (paper, plastic, PPE), miscellaneous debris / abandoned equipment, HEPA vac units, etc. from decontamination work conducted in the late 90s. The filter change waste from the service tunnels was staged on the middle floor. The housekeeping in this facility was good, and there was no evidence of speculative accumulation of any items that require additional characterization for chemical constituents or management as RCRA or TSCA regulated waste. 


\section{Chemical Contamination/Hazardous Materials of Construction}

ORNL Building 3517

September 2013

Beryllium Contamination: One of the operations conducted by Chemical Technology at ORNL was the fabrication of neutron radiation sources using beryllium powder and Am-241, Pu-238, or Pu-239 oxide. However, there is no mention throughout the historical literature of Building 3517 being one of the facilities utilized for this type of work or any other use or storage of beryllium.

Chemical Contamination of Process Systems: The report from 1960 contained procedures associated with each chemical operation performed in the facility. Each procedure required the process equipment to be emptied and flushed with water. There were also specific procedures for decontamination of the process equipment, but there was no information about how frequently this was done.

The layout of the equipment in the process cells and the arrangement of the manipulator cells was modified during the life cycle of this facility, and the exact inventory of process equipment remaining in the process cells is not clear.

The waste stream from D\&D of this building will not exhibit the characteristic of ignitability if all traces of ignitable liquids and oxidizing solutions absorbed into the process equipment components and the resulting waste stream is solid debris. Additionally, the waste stream from D\&D of this building will not exhibit the characteristic of corrosivity if all traces of corrosive liquids have been absorbed into the process equipment components and the resulting waste stream is solid debris.

The EPA has provided a regulatory clarification that the F001-listing is only appropriate when the listed solvents are used in a large-scale degreasing operation such as cold cleaning or vapor degreasing on an industrial scale. Large-scale degreasing operations were not identified in this facility. Therefore, debris from D\&D of Bldg. 3517 will not carry the EPA hazardous waste number F001.

The F003-listed solvents acetone, methanol, and xylene were not specifically listed in the early chemical inventories, but could have been used in this facility and may be present as contaminants of the process systems. However, F003-listed waste is listed solely for ignitability, and the waste stream from $\mathrm{D} \& \mathrm{D}$ of this building will not exhibit the characteristic of ignitability if all traces of solvent residues were absorbed into the process equipment components and the resulting waste stream is solid debris. Therefore, the process equipment from this building will not meet the definition of an F003-listed hazardous waste.

The operations conducted in Bldg. 3517 did not contain hazardous waste from any of the specific sources in 40 CFR 261.32, and therefore, any debris generated during D\&D of this facility will not be a K-listed waste. 


\section{HAZARDOUS MATERIALS OF CONSTRUCTION}

Hood / Hot Cell Materials of Construction Concerns: Manipulator gallery on bottom floor - 9 oil filled windows at the manipulator cells (FM stated that oil has been drained). Each set of manipulator arms is equipped with a minimum of 10 lead bricks ( 2 arms per cell, 9 cells total). The hot cells were typically equipped with a combination of incandescent spot lights and mercury vapor lamps which are conservatively assumed to still be in place. Each of the 9 manipulator cells has at least 6 switches for controlling lights.

Lead Bricks / Shielding: The lead bricks fixed to the manipulator arms are on the portion of the arms mounted on the clean side of the manipulator operating gallery.

Facility Infrastructure (Hoists, Cranes, Elevators): Crane bay on second floor is equipped with a 20-ton crane which is leaking oil from the gear box.

PCBs: The majority of mercury vapor, sodium vapor, and fluorescent lighting fixtures installed at this facility are equipped with ballasts that contain small power correction capacitors. The ballasts manufactured before 1979 contain dielectric fluid consisting of pure PCBs, typically defined as concentrations $>9,000 \mathrm{ppm}$. Fluorescent lighting ballast contains roughly one ounce of dielectric fluid, and each fluorescent fixture is typically equipped with two ballasts. The mercury vapor and sodium vapor lighting fixtures have one large ballast equipped with 2 or more three ounce capacitors.

Thermostats and/or Mercury Switches: This building was not specifically inspected for the presence of window unit air conditioners $(\mathrm{a} / \mathrm{c})$, wall mounted thermostats, or fire alarm pull boxes. However, all of these devices contain mercury and if encountered, should be removed from the facility prior to the initiation of demolition activities.

Refrigerants: The building contains several chiller units and water fountains. Additionally, a 58-ton chilled water compressor unit is located on the middle floor outside the service tunnel. All refrigeration / air conditioning equipment in this building is assumed to still contain refrigerants and oil in the compressors.

Asbestos-Containing Material (ACM): Pipe insulation throughout the facility is in good condition but was not marked to indicate if it was checked for asbestos. Floor tile throughout building is in very good condition. Documents containing estimates of ACM in place at Bldg. 3517 were not available and the Facility Manager stated that if such records were available, that he had not been issued a copy.

Lead Based Paint: The interior of Building 3517 is painted with lead-based paint. The painted surfaces were inspected during the walk down and mostly found to be in good condition with limited peeling/chipping. 
Light Bulbs: The mercury vapor, sodium vapor, fluorescent, and incandescent bulbs installed throughout this building were manufactured with toxic level of heavy metals. When subject to the TCLP, the mercury vapor and fluorescent bulbs will exceed the toxicity limits established for mercury, and sodium vapor and incandescent bulbs will exceed limits established for lead.

\section{REFERENCES}

ORNL July 1960, Hazards Report for Bldg. 3517 (Fission Product Pilot Plant), CF-60-5-26. 


\section{ORAU/ORISE \\ Process Inventory Waste Project}

\section{Chemical Contamination and Hazardous Materials of Construction ORNL Building 4507}

September 2013

Prepared by

Waste Management Innovations, Inc.

305 S. Chamberlain Avenue

Rockwood, TN 37854

865-776-5041 


\section{INTRODUCTION}

The purpose of this document is to provide an overview of the types and extent of chemical contamination and hazardous materials of concern that were observed during a recent tour of the facility. Information was also obtained by reviewing existing facility specific documentation and through discussion with the Facility Manager.

\section{CHEMICAL CONTAMINAITON}

Historical Chemical Use: Building 4507 was designed and operated as a laboratory and small-scale pilot plant utilizing the four hot cells for development studies of reactor fuel processing, separation, recovery of transuranic materials, and separation of fission products from aqueous wastes. These programs included such activities as development studies on fluoride volatility and processing of Light Water Reactor (LWR) fuels, separation of ${ }^{232} \mathrm{U}$ from ${ }^{231} \mathrm{~Pa}$, recovery of ${ }^{241} \mathrm{Am},{ }^{242} \mathrm{Cm},{ }^{243} \mathrm{Cm}$, and ${ }^{242} \mathrm{Cm}$ from plutonium and/or rare earth wastes, preparation of sol-gel microspheres of ${ }^{241} \mathrm{Am}$, ${ }^{242} \mathrm{Cm}$, and ${ }^{238} \mathrm{Pu}$, and laboratory-scale head-end processing studies of High-Temperature Gas Cooled Reactor (HTGR) and Liquid Metal Fast Breeder Reactor (LMFBR) short-cooled fuel rods. A listing of the chemicals associated with these processes could not be obtained from the facility specific information provided by the facility manager.

Chemical Storage: The chemical makeup area on the second floor was designed to contain tanks and pumps for chemical feed streams to equipment in the cells. The tanks and pumps have been removed from the chemical makeup area and the series of tanks installed above the lead floor of the hot cells have also been removed (UCOR 2013).

Currently there are no hazardous chemicals in Building 4507. Small quantities of materials that are incidental to authorized activities in Building 4507, such as cleaners, decontamination solutions; and lead shielding in packaging/shipping containers used to remove radioactive material from the facility may be used in the facility (UCOR 2013).

Waste Accumulation: The abandoned / discarded items in this facility included the following: oilfilled pumps packed in unmarked wooden shipping crates and staged on the second floor; a drill press and floor polisher staged on the east end of the hot cell access alley; wood ladders, scrap metal, bags of 'rad trash', and multiple spent, wood frame HEPA filters (2 ft x $2 \mathrm{ft} x 1 \mathrm{ft}$ ) staged on the north side of the hot cell access alley. With the exception of the oil-filled pumps in the chemical makeup area, there was no evidence of speculative accumulation of any items that require additional characterization for chemical constituents or management as RCRA or TSCA regulated waste.

Beryllium Contamination: Historical literature does not indicate any use or storage of beryllium at this facility. 
Chemical Contamination of Process Systems: The specific chemicals used in Building 4507 are not known. The waste stream from D\&D of this building will not exhibit the characteristic of ignitability if all traces of ignitable liquids and oxidizing solutions absorbed into the process equipment components and the resulting waste stream is solid debris. Additionally, the waste stream from D\&D of this building will not exhibit the characteristic of corrosivity if all traces of corrosive liquids have been absorbed into the process equipment components and the resulting waste stream is solid debris.

There is the potential that U-listed chemicals may have been used for cleaning, as a dissolution agent, or as an eluent in this facility. As a result, portions of the related process equipment (hoods, glove boxes, piping, ventilation ducts, filter media, etc.) may be contaminated with residuals of these chemicals. Debris from D\&D of these process systems would not meet the definition of a U-listed waste if the original U-listed chemicals were used as sole-active ingredients for their intended purpose.

The EPA has provided a regulatory clarification that the F001-listing is only appropriate when the listed solvents are used in a large-scale degreasing operation such as cold cleaning or vapor degreasing on an industrial scale. Large-scale degreasing operations were not identified in association with this facility. Therefore, debris from D\&D of Bldg. 4507 will not carry the EPA hazardous waste number F001.

The F003-listed solvents acetone, methanol, and xylene could have potentially been used during this facility's chemical processing operations and may be present as contaminants of the process systems. However, F003-listed waste is listed solely for ignitability, and the waste stream from D\&D of this building will not exhibit the characteristic of ignitability if all traces of solvent residues were absorbed into the process equipment components and the resulting waste stream is solid debris. Therefore, the process equipment from this building will not meet the definition of an F003-listed hazardous waste.

The operations conducted at this facility did not contain hazardous waste from any of the specific sources in 40 CFR 261.32, and therefore, any debris generated during D\&D of this facility will not be a K-listed waste.

\section{HAZARDOUS MATERIALS OF CONSTRUCTION}

Hood / Hot Cell Materials of Construction Concerns: Facility contains four hot cells on the ground level and is also equipped with a penthouse superstructure above the hot cells that contains glove boxes, and a shielded manipulator cave for small-scale work. The manipulators have been removed from the four hot cells, removed from the facility, and a non-flammable, not-explosive water-based acrylic fixative has been applied to the interior surface of the four hot cells to reduce the surface/removable contamination content within the hot cells. The hot cells were typically equipped 


\section{Chemical Contamination/Hazardous Materials of Construction}

ORNL Building 4507

September 2013

with a combination of incandescent spot lights and mercury vapor lamps which are conservatively assumed to still be in place.

Each hot cell has a $3.6 \mathrm{ft}$ thick lead glass window for viewing into the cell from the operating area. Each window was formerly filled with mineral oil which has since been drained.

Cells 1 and 4 have a lead floor and a pit containing storage tanks beneath these floors. The Cell 1 tanks are situated beneath the Cell 1 pit floor covered with approximately six inches of lead as bulk metallic shielding. The Cell 4 tanks are situated beneath the Cell 4 pit floor covered with approximately six inches of lead as bulk metallic shielding. It should be noted that the only interior items remaining today are the lead floor and the tanks beneath the floors.

The penthouse area contains a large glovebox that straddles cells 3 and 4. A second large box measuring $4.3 \mathrm{ft}$ in width $\times 6.1 \mathrm{ft}$ in length $\times 5.2 \mathrm{ft}$ in height is located at the east end of the room above Cell 1. This box is shielded with six inches of steel and equipped with a six inch thick leadglass viewing window and periscope. It's manipulators were removed during stabilization and the glove box was stabilized with fixative similar to the hot cells (UCOR 2013).

Lead Bricks / Shielding: Solid lead is present as bulk shielding material of hot cells and was discussed in the Hood / hot cell materials of concern section. The estimated lead mass as bulk metallic shielding in Cell 1 and Cell 4 is estimated to be approximately 38,228 lb based on cell floor dimensions only (UCOR 2013). Two lead pigs (4 in dia x 6 in) and two empty lead boxes (8 in x 8 in $\mathrm{x} 12$ in) are staged on first floor near the stairs.

Facility Infrastructure (Hoists, Cranes, Elevators): A 10-ton gantry crane in the penthouse is leaking oil which has migrated into the chemical makeup area located directly below the penthouse. The flooring of the chemical makeup area in the vicinity of the oil leak is heavily stained and absorbent pads are in place. Approximately four ounces of free oil was observed pooling on plastic sheeting near the absorbent pads); one hoist is installed in the cell charging area (slight oil leak from gearbox observed); one small hoist installed at chemical makeup area on second floor has oily residue visible near connection to the overhead beam; each of the four hot cell access doors is equipped with a motorized drive system (each motor has oily stains near the base of the motor's oil reservoir).

PCBs: Each of the four hot cells is equipped with two large capacitors (8 in x 8 in $\mathrm{x} 12$ in) which are mounted along the upper portion of the manipulator gallery wall. These capacitors are similar in appearance to ones installed at the Building 3030 hot cell which were determined to contain PCBs.

The majority of mercury vapor, sodium vapor, and fluorescent lighting fixtures installed at this facility are equipped with ballasts that contain small power correction capacitors. The ballasts manufactured before 1979 contain dielectric fluid consisting of pure PCBs, typically defined as concentrations $>9,000 \mathrm{ppm}$. Fluorescent lighting ballast contains roughly one ounce of dielectric

Page 3 of 4 
fluid, and each fluorescent fixture is typically equipped with two ballasts. The mercury vapor and sodium vapor lighting fixtures have one large ballast equipped with 2 or more three ounce capacitors.

Thermostats and/or Mercury Switches: Fire alarm pull boxes mounted at this location contain mercury switches. Wall mounted thermostats present on first floor to control chiller.

Refrigerants: All chillers in this building are assumed to still contain refrigerants and oil in the compressors.

Asbestos-Containing Material (ACM): Portions of steam pipe insulation on the second floor and east end of the hot cell access alley have been replaced with a silver backed insulation material labeled 'Asbestos Free'. The remaining steam pipe insulation without silver backing is not labeled, is assumed to contain asbestos, and appears to be in a non-friable state. Floor tile in the hot cell manipulator gallery was observed to be in fair condition.

Lead Based Paint: Paint in this facility is actively peeling off all horizontal and vertical surfaces. All horizontal surfaces are now littered with paint chips.

Light Bulbs: The majority of lamps still installed in this facility are fluorescent tubes. The mercury vapor, sodium vapor, fluorescent, and incandescent bulbs installed throughout this building were manufactured with toxic level of heavy metals. When subject to the TCLP, the mercury vapor and fluorescent bulbs will exceed the toxicity limits established for mercury, and sodium vapor and incandescent bulbs will exceed limits established for lead.

\section{REFERENCES}

UCOR January 2013, Hazard Assessment Document for Building 4507, High Radiation Level Chemical Development Facility, Oak Ridge National Laboratory, Oak Ridge, Tennessee, HAD-OR4507-0059, Rev. 4 


\section{ORAU/ORISE \\ Process Inventory Waste Project}

\section{Chemical Contamination and Hazardous Materials of Construction \\ ORNL Building 7500}

September 2013

Prepared by

Waste Management Innovations, Inc.

305 S. Chamberlain Avenue

Rockwood, TN 37854

865-776-5041 


\section{INTRODUCTION}

The purpose of this document is to provide an overview of the types and extent of chemical contamination and hazardous materials of concern that are assumed to be present based on the age and history of this facility. Information was obtained by reviewing existing facility specific documentation and through discussion with the Facility Manager.

\section{CHEMICAL CONTAMINAITON}

Historical Chemical Use: The Homogeneous Reactor Experiment (HRE), Bldg. 7500 was constructed in 1951, and the first aqueous experimental research reactor in the facility, HRE-l, operated until 1954. HRE-1 was fueled with a light water solution of enriched uranyl sulfate. The HRE-l process equipment and the reactor cell were decontaminated, and the equipment was removed from HRE Bldg. 7500 so that excavation could begin for below-grade cells to contain the second aqueous reactor, HRE-2. The HRE-2 reactor began operations in February 1956 and was taken offline in April 1961. In late 1961 and early 1962, the fuel and heavy water were removed from the HRE, Bldg. 7500, and the facility was sealed and placed in a standby condition. Portions of the reactor-core vessel were removed in late 1962 for study. The reactor, the chemical plant, and the auxiliary systems remain (UCOR 2013).

Cell C, adjacent to the reactor cell, contained the HRE, Bldg. 7500 Chemical Processing Plant. It is completely underground, and contains most of the equipment (i.e., liquid-solid separator, storage tank) that was used to remove and separate corrosion products from fuel and blanket systems, as well as the equipment to add additional fuel or stabilizing/corrosion-inhibiting additives to the system. This equipment was flushed multiple times with the rest of the fuel system and is now out of service (UCOR 2013).

Chemical Storage: Small quantities of standard industrial chemicals and products (e.g., glass cleaner, lubricants, solvents, etc.) and other commercially available chemicals are stored at the HRE, Bldg. 7500, in quantities below 40 CFR 302.4, Designation of Hazardous Substances, reportable quantities (RQs) or will be "standard industrial” in nature.

Waste Accumulation: The limited access to this facility prevented a tour of the waste accumulation areas. The facility manager (FM) for Building 7500 is also responsible for Buildings 3001, 3010, and 3042, and the waste accumulation areas of the other facilities were consistently well maintained with no evidence of speculative accumulation of RCRA or TSCA regulated waste.

Beryllium Contamination: There is no mention throughout the historical literature of the HRE being a beryllium moderated reactor or any other use or storage of beryllium in Building 7500. 
Chemical Contamination of Process Systems: The specific chemicals used in Building 7500 are not known. The waste stream from D\&D of this building will not exhibit the characteristic of ignitability if all traces of ignitable liquids and oxidizing solutions absorbed into the process equipment components and the resulting waste stream is solid debris. Additionally, the waste stream from D\&D of this building will not exhibit the characteristic of corrosivity if all traces of corrosive liquids have been absorbed into the process equipment components and the resulting waste stream is solid debris.

The EPA has provided a regulatory clarification that the F001-listing is only appropriate when the listed solvents are used in a large-scale degreasing operation such as cold cleaning or vapor degreasing on an industrial scale. Large-scale degreasing operations were not identified in the Research Reactor facilities. Therefore, debris from D\&D of Bldg. 7500 will not carry the EPA hazardous waste number F001.

The F003-listed solvents acetone, methanol, and xylene could have potentially been used in this facility and may be present as contaminants of the process systems. However, F003-listed waste is listed solely for ignitability, and the waste stream from D\&D of this building will not exhibit the characteristic of ignitability if all traces of solvent residues were absorbed into the process equipment components and the resulting waste stream is solid debris. Therefore, the process equipment from this building will not meet the definition of an F003-listed hazardous waste.

The operations conducted by the Research Reactor facilities did not contain hazardous waste from any of the specific sources in 40 CFR 261.32, and therefore, any debris generated during D\&D of this facility will not be a K-listed waste.

\section{HAZARDOUS MATERIALS OF CONSTRUCTION}

Hood / Hot Cell Materials of Construction Concerns: In late 1961 and early 1962, the fuel and heavy water were removed from the HRE, Bldg. 7500, and the facility was sealed and placed in a standby condition. Portions of the reactor-core vessel were removed in late 1962 for study. The reactor vessel has been sealed (UCOR 2013).

Lead Bricks / Shielding: There is no mention in the historical literature of the use of bulk lead for shielding in Building 7500.

Facility Infrastructure (Hoists, Cranes, Elevators): The steel columns in the high bay support a 20-ton crane (east-west travel) with a midbay crane (north-south travel). Both of these cranes are out of service by means of electrical isolation (UCOR 2013).

PCBs: Building 7500 was constructed in 1951. The majority of mercury vapor, sodium vapor, and fluorescent lighting fixtures installed at ORNL facilities of similar age are equipped with ballasts that 


\section{Chemical Contamination/Hazardous Materials of Construction \\ ORNL Building 7500}

September 2013

contain small power correction capacitors. Ballasts manufactured before 1979 contain dielectric fluid consisting of pure PCBs, typically defined as concentrations $>9,000 \mathrm{ppm}$. Fluorescent lighting ballast contains roughly one ounce of dielectric fluid, and each fluorescent fixture is typically equipped with two ballasts. The mercury vapor and sodium vapor lighting fixtures have one large ballast equipped with 2 or more three ounce capacitors.

This building was not specifically inspected for the presence of light fixtures that may contain PCB ballasts. However, any of the light fixtures described in this section are assumed to contain PCB capacitors and should be managed in accordance with the facility specific waste handling plan when the facility becomes available for demolition.

Thermostats and/or Mercury Switches: This building was not inspected to determine the quantity or location of window unit air conditioners $(\mathrm{a} / \mathrm{c})$, wall mounted thermostats, or fire alarm pull boxes. However, all of these devices contain mercury and if encountered, should be removed from the facility prior to the initiation of demolition activities.

Refrigerants: This building was not inspected to determine the quantity or location of chillers, window unit a/c, or drinking fountains. Any of these devices remaining in this building are assumed to still contain refrigerants and oil in the compressors and should be removed from the facility prior to the initiation of demolition activities.

Asbestos-Containing Material (ACM): This building was not inspected to determine the quantity, location or condition of ACM. However, the FM did inform the project team that all entries into this facility require the use of a full-face, air-purifying respirator because ACM has fallen off piping onto walking surfaces and the entire building is now a hazard for 'bulk friable asbestos'. Documents containing estimates of the type and condition of ACM installed at Building 7500 or results of previous asbestos surveys at this facility could not be located.

Lead Based Paint: The interior of Building 7500 is assumed to be painted with lead-based paint. The interior of this facility was not inspected to determine the condition of painted surfaces.

Light Bulbs: Building 7500 was constructed in 1951. The mercury vapor, sodium vapor, fluorescent, and incandescent bulbs installed in ORNL facilities of similar age were manufactured with toxic level of heavy metals. When subject to the TCLP, the mercury vapor and fluorescent bulbs will exceed the toxicity limits established for mercury, and sodium vapor and incandescent bulbs will exceed limits established for lead.

\section{REFERENCES}

UCOR, May 2013, Hazard Assessment Document for the Homogeneous Reactor Experiment Bldg. 7500 at the Oak Ridge National Laboratory, Oak Ridge Tennessee, HAD-OR-HRE-0019, Rev. 7. 